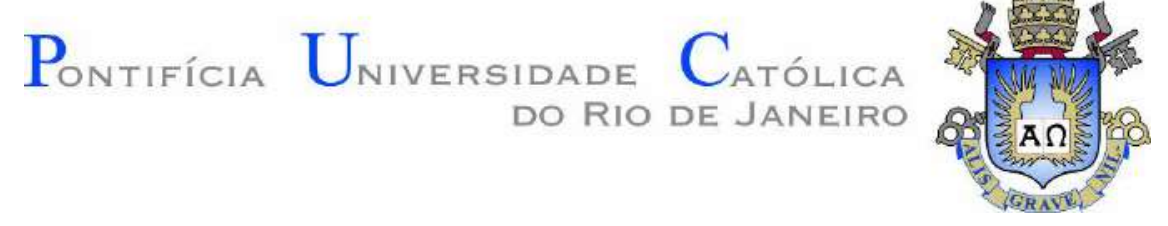

Teddy Modesto Surco Espejo

Simulation of Equatorial and Low-Latitude lonospheric Effects on the Ground-Based Augmentation System (GBAS)

Tese de Doutorado

Thesis presented to the Programa de Pós-graduação em Engenharia Elétrica of PUC-Rio in partial fulfillment of the requirements for the degree of Doutor em Engenharia Elétrica.

Advisor: Prof. Emanoel Paiva de Oliveira Costa

Rio de Janeiro

July 2020 


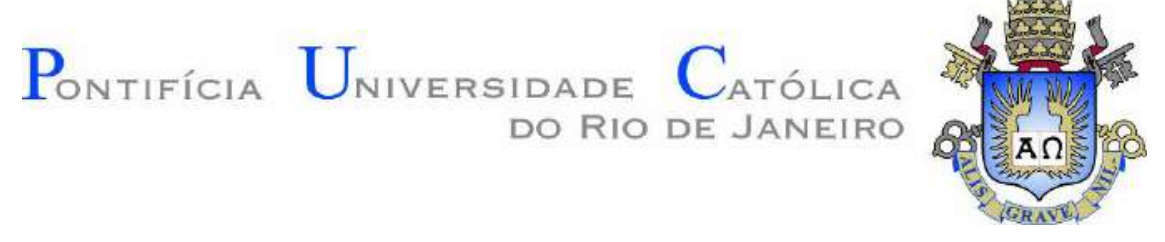

Teddy Modesto Surco Espejo

\section{Simulation of Equatorial and Low-Latitude lonospheric Effects on the Ground-Based Augmentation System (GBAS)}

Thesis presented to the Programa de Pós-graduação em Engenharia Elétrica of PUC-Rio in partial fulfillment of the requirements for the degree of Doutor em Engenharia Elétrica. Approved by the Examination Committee.

Prof. Emanoel Paiva de Oliveira Costa Advisor Centro de Estudos em Telecomunicações -PUC-Rio

Prof. Alison de Oliveira Moraes Instituto de Aeronáutica e Espaço - IAE

Prof. Eurico Rodrigues de Paula Instituto Nacional de Pesquisas Espaciais - INPE

Prof. João Francisco Galera Monico Universidade Estadual Paulista - UNESP

Prof. Flavio José Vieira Hasselmann Centro de Estudos em Telecomunicações - PUC-Rio

Prof. Luiz Alencar Reis da Silva Mello Centro de Estudos em Telecomunicações - PUC-Rio

Prof. Marco Antonio Grivet Mattoso Maia Centro de Estudos em Telecomunicações - PUC-Rio 
All rights reserved.

\section{Teddy Modesto Surco Espejo}

Received the B.S. degree in Telecommunications Engineering from the Bolivian Catholic University, Bolivia, in 2011, and the M.Sc. degree in electrical engineering from the Pontifical Catholic University of Rio de Janeiro, Brazil, in 2016. His research interests include air navigation using GBAS, ionospheric irregularities and positioning based on GNSS.

Bibliographic data

Surco Espejo, Teddy Modesto

Simulation of Equatorial and Low-Latitude Ionospheric Effects on the Ground-Based Augmentation System (GBAS) / Teddy Modesto Surco Espejo; advisor: Emanoel Paiva de Oliveira Costa. - 2020.

266 f. : il. color. ; $30 \mathrm{~cm}$

Tese (doutorado)- Pontifícia Universidade Católica do Rio de Janeiro, Departamento de Engenharia Elétrica, 2020.

Inclui bibliografia

1. Engenharia Elétrica - Teses. 2. Ionosfera. 3. GBAS. 4. GPS. 5. Navegação aérea. 6. Sinal no espaço. I. Costa, Emanoel Paiva de Oliveira. II. Pontifícia Universidade Católica do Rio de Janeiro. Departamento de Engenharia Elétrica. III. Título. 
This work is dedicated to my parents

Modesto and Hilda. 


\section{Acknowledgments}

First, I would like to thank my advisor, professor Emanoel Costa for his support, guidance and supervision during this research. Without his knowledge and assistance, this study would not have been possible and successful. His philosophy and knowledge will be present on my future academic career.

I would like to thanks to the members of my doctoral committee, Professors Alison de Oliveira Moraes, Eurico Rodrigues de Paula, João Francisco Galera Monico, Flavio José Vieira Hasselmann, Luiz Alencar Reis da Silva Mello and Marco Antonio Grivet Mattoso for their helpful suggestions.

I would like to thank my parents, Modesto and Hilda; my brothers, Juan Carlos, Bladimir, Yuly and Mijail; and my nephews for supporting and encouraging me throughout the years.

I also want to say thanks to Americo, Juliana, Johnes, Mariela, Marcelo, Mauricio and my colleagues from CETUC for their friendship and all the moments that we shared together.

I also like to thank to the project Instituto Nacional de Ciência e Tecnologia GNSS Technology for Supporting Air Navigation (INCT GNSS-NavAer) and all the research members for their interesting discussions and suggestions.

Finally, I would like to thanks to the Instituto Nacional de Pesquisas Espaciais (INPE), Rede Brasileira de Monitoramento Contínuo, operated by the Instituto Brasileiro de Geografia e Estatística (RBMC/IBGE) and the Concept for Ionospheric Scintillation Mitigation for professional GNSS in Latin America/Countering GNSS high Accuracy applications Limitations due to Ionospheric disturbances in BRAZIL (CIGALA/ CALIBRA) for their resources and providing data for this study.

This study was financed in part by the Coordenação de Aperfeiçoamento de Pessoal de Nível Superior - Brasil (CAPES) - Finance Code 001.

This work was supported in part by the Conselho Nacional de Desenvolvimento Científico e Tecnológico (CNPq) under Grant 165042/2018-4. 


\section{Abstract}

Surco Espejo, Teddy Modesto; de Oliveira Costa, Emanoel Paiva (Advisor). Simulation of Equatorial and Low-Latitude Ionospheric Effects on the Ground-Based Augmentation System (GBAS). Rio de Janeiro, 2020. 266 p. Tese de Doutorado - Departamento de Engenharia Elétrica, Pontifícia Universidade Católica do Rio de Janeiro

This research presents a study on ionospheric effects on a Ground Based Augmentation System (GBAS) in equatorial and low latitude regions. The ionosphere affects the propagation of GPS signals and can reduce the positioning accuracy in the equatorial and low-latitude regions. Auxiliary systems have been developed to meet the safety requirements of aviation. In this context, GBAS provide higher accuracy for differential corrections. To evaluate the performance of a GBAS, a simulation model of the GPS L1 signal-in-space has been developed, considering ionospheric delay based on statistical distributions of vertical Total Electron Content residuals obtained from IRI model and Rede Brasileira de Monitoramento Contínuo estimates, in combination with amplitude ionospheric scintillation simulated based on $\alpha-\mu$ probability distributions, as well as phase scintillation, generated according to empirical relationships between the indices $S_{4}$ and $\sigma_{\phi}$. The GPS L1 signal model also considers clock and random errors, tropospheric delays, ambiguity, and multipath, for a complete description. The signal in space results are injected into a GBAS ground facility simulation model, implemented to detect a varied array of possible anomalies or failures in the signal in space and to generate differential corrections based on monitoring algorithms. The GBAS generates corrections and its performance is evaluated for aircraft approaches under different ionospheric conditions at the Rio de Janeiro and Fortaleza Airports, emphasizing Approach Category I. The horizontal and vertical errors are estimated using GBAS corrections to evaluate the accuracy. The GBAS integrity is also analyzed by computing the horizontal and vertical protection levels.

\section{Keywords}

Ionosphere; GBAS; GPS; Air navigation; Signal in space. 


\section{Resumo}

Surco Espejo, Teddy Modesto; de Oliveira Costa, Emanoel Paiva (Advisor). Simulação dos Efeitos da Ionosfera Equatorial e de Baixas Latitudes no Sistema de Aumento Baseado no Solo (GBAS). Rio de Janeiro, 2020. 266 p. Tese de Doutorado - Departamento de Engenharia Elétrica, Pontifícia Universidade Católica do Rio de Janeiro

Esta tese apresenta um estudo dos efeitos ionosféricos em um Sistema de Aumento Baseado no Solo (GBAS) em regiões equatorial e de baixas latitudes. A ionosfera afeta a propagação dos sinais de GPS e pode reduzir a precisão do posicionamento nas regiões equatorial e de baixas latitudes. Sistemas auxiliares foram desenvolvidos para atender aos requisitos de segurança da aviação. Nesse contexto, o GBAS fornece maior precisão para correções diferenciais. Para avaliar o desempenho de um GBAS, um modelo de simulação do sinal-no-espaço GPS L1 foi desenvolvido, considerando o retardo ionosférico baseado nas distribuições estatísticas dos resíduos de Conteúdo Eletrônico Total vertical obtido do modelo IRI e estimativas da Rede Brasileira de Monitoramento Contínuo, em combinação com representação para a cintilação ionosférica de amplitude, simulada com base em distribuições de probabilidade $\alpha-\mu$, bem como a cintilação de fase, gerada de acordo com as relações empíricas entre os índices $S_{4}$ and $\sigma_{\phi}$. O modelo de sinal do GPS L1 também considera erros de relógios e aleatórios, retardos troposféricos, ambigüidade de ciclo e efeitos de multipercurso, para uma descrição completa. Os resultados de sinal-no-espaço são injetados em um modelo de simulação da instalação terrestre do GBAS, implementado para detectar uma variedade de possíveis anomalias ou falhas no sinal-no-espaço e para gerar correções diferenciais baseadas em algoritmos de monitoramento. O GBAS gera correções e seu desempenho é avaliado para aproximações de aeronaves em diferentes condições ionosféricas nos aeroportos do Rio de Janeiro e Fortaleza, enfatizando a Categoria de aproximação I. Os erros horizontais e verticais são estimados usando correções de GBAS para avaliar a precisão. A integridade do GBAS também é analisada calculando os níveis de proteção horizontal e vertical.

\section{Palavras-chave}

Ionosfera; GBAS; GPS; Navegação aérea; Sinal no espaço. 


\section{Table of Contents}

1. INTRODUCTION 27

$\begin{array}{ll}\text { 1.1. Motivation } & 27\end{array}$

1.2. Research Objectives $\quad 29$

1.3. Description of the Following Chapters 30

2. GLOBAL POSITIONING SYSTEM AND AUGMENTATION SYSTEMS

2.1. GPS 32

2.1.1. GPS Observable Error Sources 34

2.2. Air Navigation Using GPS $\quad 37$

2.3. Augmentation Systems 38

2.3.1. Satellite-Based Augmentation System (SBAS) 38

2.3.2. Ground-Based Augmentation System (GBAS) 39

3. ESTIMATION AND ANALYSIS OF TEC USING GPS MEASUREMENTS AND IRI MODEL PREDICTIONS 45

3.1. Ionosphere 45

3.1.1. Variations in the lonosphere 46

3.1.2. Ionospheric Irregularities $\quad 48$

3.2. Ionospheric Refraction 49

3.3. Extraction of Absolute TEC from GPS Observations 50

3.3.1. TEC from Pseudorange Observation 50

3.3.2. TEC from Carrier Phase Measurement 52

3.3.3. Correction of Cycle Slips 53

3.3.4. Leveling of the Carrier Phase with the Pseudorange 56

3.3.5. Absolute sTEC

3.3.6. Differential Code Biases $\quad 57$

3.3.7. Absolute vTEC $\quad 59$

3.4. Ionospheric Pierce Point 60

$\begin{array}{ll}\text { 3.5. Data } & 60\end{array}$ 
3.5.1. Geomagnetic and Solar indices 62

3.5.2. GPS Satellite positions 63

3.6. Results 64

3.6.1. Estimation of Vertical TEC 64

3.6.2. Detection and Repair of Cycle Slips 66

3.6.3. Results for Differential Code Biases of the Receivers 68

3.6.4. Maximum Daily Vertical TEC 72

3.6.5. Comparison Between vTEC Measurements and

Corresponding Predictions by the International Reference

$\begin{array}{ll}\text { Ionosphere Model for the Brazilian Region } & 74\end{array}$

\section{ESTIMATION AND ANALYSIS OF IONOSPHERIC}

\section{GRADIENTS}

4.1. Ionospheric Gradient Estimation 82

4.2. Results of the Vertical Ionospheric Delay Gradient

Using the Time-Step Method 84

4.3. Daily Maximum Vertical Ionospheric Delay Gradient

Using the Time-Step Method 86

4.4. Results of the Vertical lonospheric Delay Gradient

using the Station-Pair Method 88

4.5. Time Variation of the Daily Maximum Vertical

Ionospheric Gradient using the Station-Pair Method 97

5. SIGNAL IN SPACE $\quad 100$

5.1. Pseudorange, Carrier phase and Received Signal Model 100

$\begin{array}{ll}\text { 5.1.1. Geometric Range } & 101\end{array}$

5.1.2. Satellite Clock Error 102

$\begin{array}{ll}\text { 5.1.3. Ionospheric Delay } & 103\end{array}$

5.1.4. Tropospheric Delay 104

$\begin{array}{ll}\text { 5.1.5. Multipath } & 108\end{array}$

5.1.6. Amplitude and Phase Scintillation 114

$\begin{array}{ll}\text { 5.1.7. Cycle ambiguity } & 121\end{array}$

5.1.8. Effective Isotropic Radiated Power 124

5.1.9. Receiver Clock Errors and Random Errors 124 
5.2. Results from the Pseudorange, Carrier Phase and

Received Signal Models 125

5.2.1. Geometric Distance 127

5.2.2. Satellite Clock Error 128

5.2.3. Ionospheric Delay 129

5.2.4. Tropospheric Delay 131

5.2.5. Multipath 132

5.2.6. Amplitude Scintillation 134

5.2.7. Phase Scintillation 138

5.2.8. Time Series of the Pseudorange 139

5.2.9. Time Series of the Carrier Phase 141

5.2.10. Time Series of the Received Power 143

5.3. Definition of Active Channels Based on the

CALIBRA/CIGALA Network 144

6. GROUND BASED AUGMENTATION SYSTEM SIMULATION MODEL 154

6.1. Signal-in-Space Receive and Decode (SISRAD) 156

6.2. Signal Quality Receiver (SQR) 156

6.3. Smooth 157

6.4. Measurement Quality Monitoring (MQM) 157

6.5. Signal Quality Monitoring (SQM) 161

6.6. Data Quality Monitoring (DQM) 164

6.7. Executive Monitor (EXM) 166

6.8. First Stage of the Executive Monitor (EXM-I) 167

6.9. Correction 172

6.10. Average 173

6.11. Multiple Reference Consistency Check (MRCC) 174

6.12. $\sigma-\mu$ Monitor 176

6.13. Message Field Range Test (MFRT) 178

6.14. Second Stage of the Executive Monitor (EXM-II) 179

6.15. Protection Levels 180

6.15.1. Airborne Pseudorange Error 183

6.15.2. Differential Tropospheric Delay Error 184 
6.15.3. Ionospheric Residual Uncertainty

6.16. Very High Frequency (VHF) Data Broadcast (VDB)

Transmitter

185

6.17. Estimation of the Aircraft Position

185

7. RESULTS FROM THE GBAS SIMULATION MODEL

7.1. Aircraft Positioning at the Rio de Janeiro/Galeão

Antonio Carlos Jobim International Airport, Brazil

191

7.1.1. Scenario

192

7.1.2. Flight Data

193

7.1.3. Case Study: Rio de Janeiro Airport, 20 December 2014

195

7.1.4. Case Study: Rio de Janeiro Airport, 22 July 2009

225

7.2. Aircraft Positioning at the Fortaleza Airport, Brazil 236

7.2.1. Scenario

236

7.2.2. Flight Data

237

7.2.3. Case study: Fortaleza Airport, 20 December 2014

238

8. CONCLUSIONS AND FUTURE WORKS

249

8.1. Conclusions

249

8.2. Future works

251

Bibliography 


\section{List of Figures}

Figure 2.1: GPS satellite network. 33

Figure 2.2: WGS84 coordinate system. $\quad 34$

Figure 2.3: Differential GPS. 38

Figure 2.4: SBAS network. $\quad 39$

Figure 2.5: Differential correction of GBAS. 40

Figure 2.6: Block diagram of the GBAS Ground Facility (GGF) model. $\quad 41$

Figure 2.7: Alert limits and protections levels. 43

Figure 3.1: Vertical profile of the ionosphere. 46

Figure 3.2: a) lonospheric regions of the world; b) Sunspot number. $\quad 47$

Figure 3.3: Effects of ionospheric refraction on GPS signals at different frequencies. 50

Figure 3.4: Geometry of a GPS satellite, the ionosphere and a receiver.

Figure 3.5: Positions of the ground-based RBMC stations in 2002, 2008, and 2013.

Figure 3.6: Map of geomagnetic DIP latitudes. 62

Figure 3.7: Solar and geomagnetic indices (F10.7 and Kp indices). 63

Figure 3.8: Positions of all the ground-based RBMC stations available in 2008; path positions of the 400-km IPPs for the IMPZ station and each GPS satellite on September 11, 2002.

Figure 3.9: Detection of cycle slips for the BRAZ station on 11 September 2002.

Figure 3.10: Cycle slip repaired for the BRAZ station on 11 September 2002

Figure 3.11: $D C B$ estimated through the combined methods and CODE method for the BRAZ station in 2002, 2008 and 2013.

Figura 3.12: GPS VTEC for IMPZ, BRAZ and RIOD stations on September 11, 2002 and September 10, 2008 respectively.

Figure 3.13: Skyplot of GPS satellite orbits on September 11, 2002.

Figure 3.14: Maximum daily values of VTEC for the IMPZ, BRAZ, and RIOD stations in 2002, 2008 and 2013. 
Figura 3.15: RBMC and IRI VTEC for the IMPZ, BRAZ, and RIOD stations on 11 September 2002 and 10 September 2008.

Figure 3.16: Histograms of residuals (VTEC $\mathrm{RBMC}_{\mathrm{R}}-\mathrm{VTEC} \mathrm{C}_{\mathrm{RI}}$ ).

Figure 3.17: Histograms and CDF for the classes 18 [geomagnetic latitude of IPP: $\left(-4^{\circ},+4^{\circ}\right)$ ], 42 [geomagnetic latitude of IPP: $\left(-4^{\circ}-12^{\circ}\right)$ or $\left(+4^{\circ},+12^{\circ}\right)$ ] and 66 [geomagnetic latitude of IPP: $\left(-12^{\circ},-22^{\circ}\right)$ ]. Fixed parameter ranges: local time (21:30 LT - 01:30 LT); EPB season (September-March); solar activity (F10.7>150); and geomagnetic activity $(\mathrm{Kp}>4)$.

Figure 4.1: Station-pair method.

Figure 4.2: Time-step method.

Figure 4.3: CCDF of the vertical ionospheric delay gradients estimated using the time-step method based on data from IMPZ, BRAZ and RIOD stations in 2002.

Figure 4.4: CCDF of the vertical ionospheric delay gradients estimated using the time-step method based on data from IMPZ, BRAZ and RIOD stations in 2008.

Figure 4.5: CCDF of the vertical ionospheric delay gradients estimated using the time-step method based on data from IMPZ, BRAZ and RIOD stations in 2013.

Figure 4.6: Time variation of the daily maximum vertical ionospheric delay gradients for the IMPZ, BRAZ and RIOD stations in 2002, 2008 and 2013.

Figure 4.7: Positions of ground-based RBMC station-pairs in 2013.

Figure 4.8: Vertical ionospheric delays computed for each GPS satellite and station SSA1; and SAVO on 10 November 2013.

Figure 4.9: Vertical ionospheric delay gradient computed between the two stations SAVO and SSA1 on 10 November 2013.

Figure 4.10: Estimated vertical ionospheric delay; corresponding vertical ionospheric delay gradient, associated with satellite 18 and the two stations SAVO and SSA1 on 10 November 2013.

Figure 4.11: CCDFs of the vertical ionospheric delay gradients estimated by the station-pair method based on data from the combinations of stations: ONRJ-RIOD and SSA1-SAVO in 2008. 
Figure 4.12: CCDFs of the vertical gradients estimated by the station-pair method based on data from the combinations of stations: ONRJ-RIOD, CEEU-CEFT, and SSA1-SAVO in 2013.

Figure 4.13: Daily values of the mean and standard deviation of VIG estimated by the station-pair method computed for SSA1 and SAVO stations; RIOD and ONRJ stations in 2008.

Figure 4.14: Daily values of the mean and standard deviation of VIG estimated by the station-pair method for CEEU and CEFT stations; SSA1 and SAVO stations; RIOD and ONRJ stations in 2013.

Figure 4.15: Daily maximum VIG estimated by the station-pair method based on data from the combinations of stations: ONRJRIOD and SSA1-SAVO in 2008.

Figure 4.16: Daily maximum VIG estimated by the station-pair method based on data from the combinations of stations: ONRJRIOD, SSA1-SAVO and CEEU-CEFT in 2013.

Figure 4.17: CCDFs of the vertical gradients estimated by the timestep method and station-pair method based on data from RIOD station and ONRJ-RIOD pair in 2013, respectively.

Figure 5.1: Tropospheric delay as a function of elevation angle estimated for Rio de Janeiro receiver station (DoY $=256$, humidity $=$ $80 \%$, temperature $\mathrm{t}=25^{\circ} \mathrm{C}$, atmospheric pressure $=1012 \mathrm{hPa}$ ).

Figure 5.2: Two-ray ground reflection model.

Figure 5.3: Depolarization of the signal due to multipath.

Figure 5.4: Magnitude and phase (horizontal and vertical polarization) of the Fresnel reflection coefficients using the electrical properties of wet ground $\left(\epsilon_{r}=30, \sigma_{g}=0.01 \mathrm{~S} / \mathrm{m}\right)$.

Figure 5.5: (a) and (b) Two signal records illustrating the differences between the occurrences of fade depths for signals with approximately the same scintillation index $S_{4}$ and decorrelation time $\tau_{o}$; (c) Corresponding measured occurrences and the best-fit $\alpha-\mu$ distributions, as well as the Nakagami- $m$ distribution with $m=1 / S_{4}^{2}=1 / 0.7^{2}=2.04$.

Figure 5.6: Block diagram illustrating a straightforward mechanization of the proposed scintillation model.

Figure 5.7: Estimated power spectral density from simulated data $\left(S_{4}=0.6\right.$ and $\left.\tau_{0}=0.2\right)$ and the second-order Butterworth model. 
Figure 5.8: Empirical probability distribution functions of $\alpha$ for differrent values of $S_{4}$ intervals $\left(S_{4}-0.05, S_{4}+0.05\right)$, locations, and for the GPS L1 signal.

Figure 5.9: Results of $F_{R}(r)$ as a function of signal intensity, assuming four fixed values of $S_{4}(0.7,0.8,0.9$ and 1.0$)$ and different a values.

Figure 5.10: a) Average values for $S_{4}$ with standard-deviation bars, as functions of $\sigma_{\varphi}$. b) Complementary cumulative distribution functions of $\sigma_{\varphi}$ for the $\mathrm{L} 1$ signal and different $\mathrm{S}_{4}$ ranges.

Figure 5.11: Cumulative distribution and complementary cumulative distribution of ambiguity value; and cumulative distribution and commentary cumulative distribution of time between consecutive cycle slips for the Rio de Janeiro RBMC station during the year 2014.

Figure 5.12: Positions of the Rio de Janeiro receiver and RBMC stations, as well as the geomagnetic equator.

Figure 5.13 Estimated geometric distances between the Rio de Janeiro station and GPS satellites on the night of 19-20 December 2014.

Figure 5.14: Estimated elevation angle from the Rio de Janeiro station to each visible GPS satellites on the night of 19-20 December 2014.

Figure 5.15: Estimated satellite clock error for each GPS satellite tracked by Rio de Janeiro station on the night of 19-20 December 2014.

Figure 5.16: Estimated ionospheric delays between GPS satellites and the Rio de Janeiro station on the night of 19-20 December 2014.

Figure 5.17: Estimated ionospheric delay between GPS satellites and the Rio de Janeiro Station on the night of 21-22 July 2014.

Figure 5.18: Estimated tropospheric delay between GPS satellites and the Rio de Janeiro station on the night of 19-20 December 2014.

Figure 5.19: Estimated code, carrier phase, and received power multipath errors due to one rough plane reflector for GPS L1 signals, considering a two-ray model.

Figure 5.20: One-min time series of amplitude scintillation simulated for the Rio de Janeiro station and satellites SV14, SV16, SV18, SV21, SV22, and SV27 during the time interval 01:00 UTC to 01:01 UTC on 20 December 2014, assuming the $\left(S_{4}, \alpha, \mu\right)$ values displayed 
in the associated legends; power spectral densities of the corresponding amplitude scintillation.

Figure 5.21: Frequency distribution of simulated data and theoretical $\alpha-\mu$ probability density functions for the simulated time series of of amplitude scintillation.

Figure 5.22: One-min time series of amplitude scintillation simulated for the Fortaleza station and satellites SV14, SV16, SV18, SV21, SV22 and SV27 during the time interval 01:00 UTC to 01:01 UTC on 20 December 2014, assuming the $\left(S_{4}, \alpha, \mu\right)$ values; power spectral densities of the corresponding amplitude scintillation.

Figure 5.23: One-min time series of amplitude scintillation simulated for the Rio de Janeiro station and satellites SV14, SV16, SV18, SV21, SV22, and SV27 during the time interval 01:00 UTC to 01:01 UTC on 22 July 2014 , assuming the $\left(S_{4}, \alpha, \mu\right)$ values; power spectral densities of the corresponding amplitude scintillation.

Figure 5.24: One-min time series of phase scintillation simulated for the Rio de Janeiro station and satellites SV14, SV16, SV18, SV21, SV22, and SV27 during the time interval 01:00 UTC to 01:01 UTC on 20 December 2014 , assuming the $\left(S_{4}, \sigma_{\varphi}\right)$ values.

Figure 5.25: Time series for the pseudorange simulated for the Rio de Janeiro Station and several satellites on the night of 19-20 December 2014.

Figure 5.26: Time series of associated effects (ionospheric and tropospheric delays, receiver clock, multipath, and random errors) simulated for Rio de Janeiro station and different satellites on the night of 19-20 December 2014.

Figure 5.27: Time series of carrier phase simulated for Rio de Janeiro station and several satellites on the night of 19-20 December 2014.

Figure 5.28: Time series of associated effects (ionospheric and tropospheric delays, cycle ambiguity, receiver clock, multipath, and random errors) simulated for Rio de Janeiro station and different satellites on the night of 19-20 December 2014.

Figure 5.29: Time series of the received signal power for the Rio de Janeiro station and satellites SV14, SV16, SV18, SV21, SV22 and 
SV27 during the time interval 01:00 UTC to 01:10 UTC on the night of 20 December 2014.

Figure 5.30: Positions of Palmas, Presidente Prudente, São José dos Campos and Porto Alegre receiver stations, with respect to the geomagnetic equator and the southern crest of the EIA.

Figure 5.31: Empirical distributions of occurrences of visible and active GPS satellites tracked by the PALM station (Palmas, dip latitude: $-7.78^{\circ}$ ) for different combinations of seasons and solar activities.

Figure 5.32: Empirical distributions of occurrences of visible and active GPS satellites tracked by the PRU1 and PRU2 stations (Presidente Prudente, dip latitude: $-16.01^{\circ}$ ) for different combinations of seasons and solar activities.

Figure 5.33: Empirical distributions of occurrences of visible and active GPS satellites tracked by the SJCI, SCJU and SJCE stations (São José dos Campos, dip latitude: $-19.82^{\circ}$ ) for different combinations of seasons and solar activities.

Figure 5.34: Empirical distributions of occurrences of visible and active GPS satellites tracked by POAL station (Porto Alegre, dip latitude: $-22.01^{\circ}$ ) for different combinations of seasons and solar activities.

Figure 5.35: Empirical cumulative distribution of the number of visible and active GPS satellites tracked by the PALM station (Palmas, dip latitude: $-7.78^{\circ}$ ), according to combinations of season and solar activity ranges.

Figure 5.36: Empirical cumulative distribution of the number of visible and active GPS satellites tracked by the PRU1 and PRU2 station (Presidente Prudente, dip latitude: $-16.01^{\circ}$ ), according to combinations of season and solar activity ranges.

Figure 5.37: Empirical cumulative distribution of the number of visible and active GPS satellites tracked by the SJCI, SCJU and SJCE station (São José dos Campos, Dip latitude: -19.82), according to combinations of season and solar activity range.

Figure 5.38: Empirical cumulative distribution of the number of visible and active GPS satellites tracked by POAL station (Porto Alegre, Dip latitude: $-22.01^{\circ}$ ), according to combinations of season and solar activity ranges. 
Figure 5.39: Empirical distribution of classes of satellite-receiver pairs with different intervals of $\mathrm{S}_{4}$ values, for different combinations of season and solar activity, considering 6 visible and active satellites, based on measurements from the three stations located in São José dos Campos.

Figure 6.1: Block diagram of the GBAS Ground Facility model.

Figure 7.1: Positions of the GBAS station and reference antennas at the Rio de Janeiro Airport.

Figure 7.2: Last section of TAM/JJ3839 flight path.

Figure 7.3: Aircraft speed and aircraft altitude of flight TAM/JJ3839 between 08:52:34 UTC and 08:57:34 UTC.

Figure 7.4: GPS satellite skyplot for TAM/JJ3839 flight from 00:00:00 UTC to 00:10:00 UTC on 20 December 2014; ground projections of the GPS satellite positions and the corresponding IPPs.

Figure 7.5: Time series of amplitude scintillation index $\mathrm{S}_{4}$ for each GPS satellite, with 1-min resolution.

Figure 7.6: Time series of standard deviation of phase scintillation $\sigma_{\varphi}$ for each GPS satellite, with 1-min resolution.

Figure 7.7: Time series of the effects due to ionospheric and tropospheric delays, multipath and random errors on the pseudorange, generated for TAM/JJ3839 flight from 00:00:00 UTC and 00:10:00 UTC on 20 December 2014.

Figure 7.8: Time series of the effects due to ionospheric and tropospheric delays, multipath, phase ambiguity, phase scintillation and random errors on the carrier phase, generated for TAM/JJ3839 flight from 00:00:00 UTC and 00:10:00 UTC on 20 December 2014.

Figure 7.9: Time series of the received signal power, generated for TAM/JJ3839 flight from 00:00:00 UTC and 00:10:00 UTC on 20 December 2014, considering the effects due to amplitude scintillation, multipath, and random errors.

Figure 7.10: $|R|+R e s i d u a l$ TEC Map and ground projections of the corresponding IPPs of each satellite at 00:10:00 UTC on 20 December 2014.

Figure 7.11: Time series of the phase scintillation, generated for TAM/JJ3839 flight from 00:00:00 UTC and 00:10:00 UTC on 20 December 2014. 
Figure 7.12: NED positioning errors; Horizontal and Vertical positioning errors; all based on pseudoranges generated during TAM/JJ3839 flight from 00:00:00 UTC to $00: 10: 00$ UTC on 20 December 2014. 204

Figure 7.13: MQM Carrier Acceleration test results for the RSMU1 GBAS receiver during TAM/JJ3839 flight from 00:00:00 UTC to 00:10:00 UTC on 20 December 2014.

Figure 7.14: MQM Carrier Step test results for the RSMU1 GBAS receiver during TAM/JJ3839 flight from 00:00:00 UTC to 00:10:00 UTC on 20 December 2014.

Figure 7.15: MQM Carrier-Smoothed Code Innovations test results for the RSMU1 GBAS receiver during TAM/JJ3839 flight from 00:00:00 UTC to 00:10:00 UTC on 20 December 2014.

Figure 7.16: SQM Correlation Peak Symmetry test results for the RSMU1 GBAS receiver during TAM/JJ3839 flight from 00:00:00 UTC to 00:10:00 UTC on 20 December 2014.

Figure 7.17: SQM Code-Carrier Divergence test results for the RSMU1 GBAS receiver during TAM/JJ3839 flight from 00:00:00 UTC to 00:10:00 UTC on 20 December 2014.

Figure 7.18: SQM Received Signal Power test results for the RSMU1 GBAS receiver during TAM/JJ3839 flight from 00:00:00 UTC to 00:10:00 UTC on 20 December 2014.

Figure 7.19: DQM Yesterday Ephemerides minus Today Ephemerides (Ye-Te) test results for the RSMU1 GBAS receiver during TAM/JJ3839 flight from 00:00:00 UTC to 00:10:00 UTC on 20 December 2014.

Figure 7.20: Unavailable GPS satellites for the RSMU1 GBAS receiver during TAM/JJ3839 flight from 00:00:00 UTC to 00:10:00 UTC on 20 December 2014.

Figure 7.21: MRCC B-values test results for the RSMU1 GBAS receiver during TAM/JJ3839 flight from 00:00:00 UTC to 00:10:00 UTC on 20 December 2014.

Figure 7.22: MFRT test result for the GBAS system during TAM/JJ3839 flight from 00:00:00 UTC to 00:10:00 UTC on 20 December 2014. 
Figure 7.23: Time series of the pseudoranges and smoothed pseudoranges, excluding the respective geometric distances and satellite clock errors, generated for TAM/JJ3839 flight from 00:00:00 UTC to 00:10:00 UTC on 20 December 2014.

Figure 7.24: Pseudorange corrections estimated by the GBAS during TAM/JJ3839 flight from 00:00:00 UTC to 00:10:00 UTC on 20 December 2014.

Figure 7.25: NED positioning errors; Horizontal and Vertical positions errors of TAM/JJ3839 flight based on corrected pseudoranges from 00:00:00 UTC to 00:10:00 UTC on 20 December 2014.

Figure 7.26: Horizontal positioning error and horizontal protection level of TAM/JJ3839 flight from 00:00:00 UTC to 00:10:00 UTC on 20 December 2014.

Figure 7.27: Vertical positioning error and vertical protection level of TAM/JJ3839 flight from 00:00:00 UTC to 00:10:00 UTC on 20 December 2014.

Figure 7.28: Integrity plot

Figure 7.29: Integrity plot of the horizontal performance of TAM/JJ3839 flight for CAT-I from 00:00:00 UTC to 00:10:00 UTC on 20 December 2014

Figure 7.30: Integrity plot of the vertical performance of TAM/JJ3839 flight for CAT-I from 00:00:00 UTC to 00:10:00 UTC on 20 December 2014.

Figure 7.31: Integrity plot of vertical performance of TAM/JJ3839 flight for CAT-I and APV from 00:00:00 UTC to 00:10:00 UTC on 20 December 2014.

Figure 7.32: GPS satellite skyplot for TAM/JJ3839 flight from 00:00:00 UTC to 00:10:00 UTC on 22 July 2009; ground projections of the GPS satellite positions and the corresponding IPPs.

Figure 7.33: Time series of amplitude scintillation index $S_{4}$ for each GPS satellite, with 1-min resolution.

Figure 7.34: Time series of standard deviation of phase scintillation $\sigma_{\varphi}$ for each GPS satellite, with 1-min resolution.

Figure 7.35: Time series of the effects due to ionospheric and tropospheric delays, multipath and random errors on the 
pseudorange, generated for TAM/JJ3839 flight from 00:00:00 UTC to $00: 10: 00$ UTC on 22 July 2009 .

Figure 7.36: Time series of the received signal power, generated for TAM/JJ3839 flight from 00:00:00 UTC to 00:10:00 UTC on 22 July 2009 , considering the effects due to amplitude scintillation, multipath, and random errors.

Figure 7.37: IRI+Residual TEC Map and ground projections of the corresponding IPPs at 00:10:00 UTC on 22 July 2009.

Figure 7.38: Pseudorange corrections estimated by the GBAS during TAM/JJ3839 flight from 00:00:00 UTC to 00:10:00 UTC on 22 July 2009.

Figure 7.39: NED positioning errors based on uncorrected and GBAS corrected pseudoranges during TAM/JJ3839 flight from 00:00:00 UTC to 00:10:00 UTC on 22 July 2009.

Figure 7.40: Horizontal positioning error and horizontal protection level for TAM/JJ3839 flight from 00:00:00 UTC to 00:10:00 UTC on 22 July 2009.

Figure 7.41: Vertical positioning error and vertical protection level for TAM/JJ3839 flight from 00:00:00 UTC to 00:10:00 UTC on 22 July 2009.

Figure 7.42: Integrity plot of the horizontal performance of TAM/JJ3839 flight for CAT-I from 00:00:00 UTC to 00:10:00 UTC on 22 July 2009.

Figure 7.43: Integrity plot of vertical performance of TAM/JJ3839 flight for CAT-I from 00:00:00 UTC to 00:10:00 UTC on 22 July 2009.

Figure 7.44: Simulated positions of GBAS monitor and reference antennas at Pinto Martins Airport (Fortaleza, Brazil).

Figure 7.45: Path of the last section of AZU5346 flight. 238

Figure 7.46: GPS satellite skyplot for AZU5346 flight from 00:00:00 UTC to 00:10:00 UTC on 20 December 2014; ground projections of the corresponding IPPs.

Figure 7.47: Time series of amplitude scintillation index $S_{4}$ for each GPS satellite, with 1-min resolution.

Figure 7.48: Time series of standard deviation of phase scintillation $\sigma_{\varphi}$ for each GPS satellite, with 1-min resolution. 
Figure 7.49: Time series of the effects due to ionospheric and tropospheric delays, multipath and random errors on the pseudorange, generated for AZU5346 flight from 00:00:00 UTC to 00:10:00 UTC on 20 December 2014.

Figure 7.50: Time series of the received signal power, generated for AZU5346 flight from 00:00:00 UTC to 00:10:00 UTC on 20 December 2014, considering the effects due to amplitude scintillation, multipath, and random errors.

Figure 7.51: Pseudorange corrections estimated by the GBAS during AZU5346 flight from 00:00:00 UTC to 00:10:00 UTC on 20 December 2014.

Figure 7.52: NED positioning errors based on uncorrected and GBAS-corrected pseudoranges during AZU5346 flight from 00:00:00 UTC to 00:10:00 UTC on 20 December 2014.

Figure 7.53: Horizontal positioning error and horizontal protection level for AZU5346 flight from 00:00:00 UTC to 00:10:00 UTC on 20 December 2014.

Figure 7.54: Vertical positioning error and vertical protection level for AZU5346 flight from 00:00:00 UTC to 00:10:00 UTC on 20 December 2014.

Figure 7.55: Integrity plot of the horizontal performance of AZU5346 flight for CAT-I from 00:00:00 UTC to 00:10:00 UTC on 20 December 2014.

Figure 7.56: Integrity plot of vertical performance of AZU5346 flight for CAT-I from 00:00:00 UTC to 00:10:00 UTC on 20 December 2014. 


\section{List of Tables}

Table 2.1: Requirements for precision approach and landing. $\quad 44$

Table 3.1: Solar and geomagnetic indices for selected days. 64

Table 3.2: Description of receiver stations. 65

Table 3.3: Statistics of estimated DCB. 68

Table 3.4: Combinations of geophysical parameters. 77

Table 3.5: Means and standards deviation of the 72 statistical 81 distributions.

Table 4.1: Absolute maximum values of VIG in 2002, 2008 and $2013 \quad 88$

Table 4.2: Description of station pairs. $\quad 89$

Table 4.3: Largest slant ionospheric gradients estimated for the pair:

SAVO-SSA1 stations in 2013 and 2014.

Table 5.1: Coefficients of the Niell hydrostatic mapping function

(average values). 106

Table 5.2: Amplitude coefficients of the Niell hydrostatic mapping function.

Table 5.3: Coefficients of the Niell hydrostatic mapping function (height correction). 107

Table 5.4: Coefficients of the Niell wet mapping function. 107

Table 5.5: Probabilities of $S_{4}$ occurrences in different intervals $\left(S_{4}-0.05, S_{4}+0.05\right)$ and locations for the L1 signal.

Table 5.6: Average values and standard deviations of $\alpha$ in different $S_{4}$ intervals $\left(S_{4}-0.05, S_{4}+0.05\right)$ and locations for the $L 1$ signal.

Table 5.7: Season and solar activity parameters considered for cycle ambiguity.

Table 5.8: Geophysical parameters on 19-20 December 2014 . 126

Table 5.9: Description of the Rio de Janeiro station. 126

Table 5.10: Simulation system parameters. 126

Table 5.11: Description of the CIGALA/CALIBRA receiver stations. 145

Table 5.12: Season and solar activity parameters. 145 
Table 5.13: Probabilities of the number of visible GPS satellites for each of the four receiver stations of the CIGALA/CALIBRA network.

Table 5.14: Amplitude scintillation intervals and their representations. 151

Table 6.1: GBAS data interfaces.

Table 6.2: Tracking matrix $\mathrm{T}(k)$.

Table 6.3: Quality matrix $Q(k)$. 168

Table 6.4: Matrix $Q^{\prime}(k)$. 169

Table 6.5: Decision matrix $\mathrm{D}(k)$. 170

Table 6.6: Decision matrix $\mathrm{ED}(k)$. 170

Table 6.7: Isolate cases and actions.

Table 6.8: Matrix $D_{p r}(k)$.

Table 6.9: Ground Facility Error Allocation Model.

Table 6.10: Matrix $\operatorname{EIID}(k)$.

Table 6.11: Value of the multiplier $K_{f f m d}$.

Table 6.12: Airborne error model parameters.

Table 7.1: Positions of receiver antennas at the Rio de Janeiro Airport.

Table 7.2: Information on the TAM/JJ3839 flight.

Table 7.3: Geophysical parameters on 20 December 2014.

Table 7.4: Environment parameters at the Rio de Janeiro Airport on 20 December 2014.

Table 7.5: Simulation system parameters.

Table 7.6: Accuracy precision approach.

Table 7.7: Geophysical parameters on 22 July 2009.

Table 7.8: Environmental parameters at the Rio de Janeiro Airport on 22 July 2009.

Table 7.9: Positions of reference antennas at Fortaleza Airport.

Table 7.10: Information on the AZU5346 flight.

Table 7.11: Environment parameters at the Fortaleza Airport on 20 December 2014. 


\section{List of Abbreviations and Acronyms}

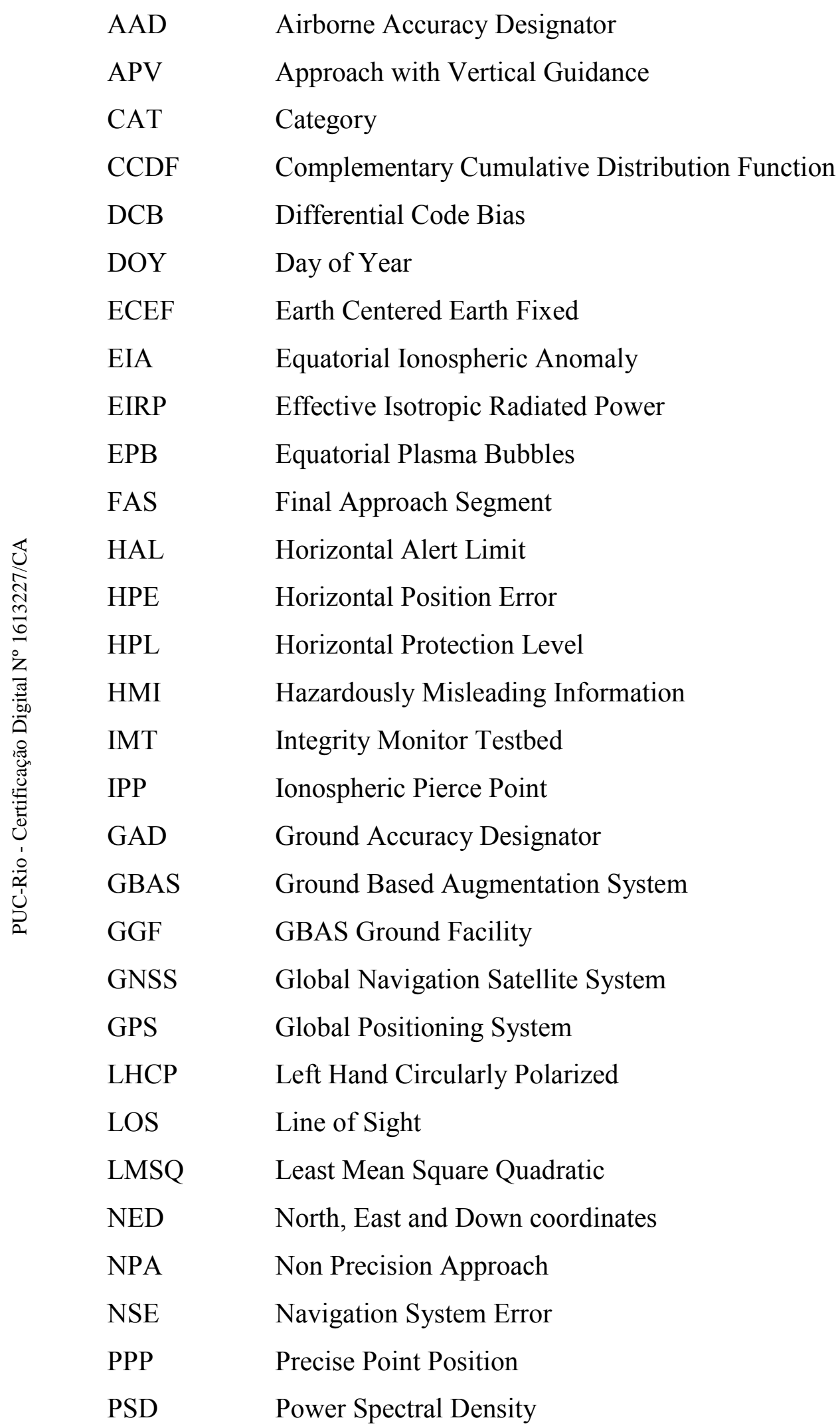




\begin{tabular}{|c|c|}
\hline & Right Hand Circularly Polarized \\
\hline RNP & Required Navigation Performance \\
\hline RSMU & Remote Satellite Measurement Unit \\
\hline RTS & Real Time Service \\
\hline SBAS & Satellite Based Augmentation System \\
\hline SFU & Solar Flux Unit \\
\hline SIS & Signal in Space \\
\hline SNR & Signal to Noise Ratio \\
\hline SV & Satellite Vehicle \\
\hline TEC & Total Electron Content \\
\hline TID & Traveling Ionospheric Disturbances \\
\hline UTC & Universal Time Coordinated \\
\hline VHF & Very High Frequency \\
\hline VDB & Very High Frequency Data Broadcast \\
\hline VIG & Vertical Ionospheric Gradient \\
\hline VAL & Vertical Alert Limit \\
\hline VPE & Vertical Position Error \\
\hline VPL & Vertical Protection Level \\
\hline WGS & World Geodetic System \\
\hline
\end{tabular}




\section{Introduction}

\subsection{Motivation}

Ionospheric effects on radio wave propagation is a relevant topic to various study areas, including communication, radionavigation, and space-based observation systems, as well as space weather studies (Parkinson and Gilbert, 1983; Liu and Gao, 2004). The ionosphere is one of the most important sources of error in positioning and navigation systems based on Global Navigation Satellite Systems (GNSS), such as the Global Positioning System (GPS). The ionosphere is a dispersive medium for GNSS signals propagating between satellites and ground receivers. One of the largest error source for GPS-based applications is the delay produced by the ionosphere (code or carrier-phase measurements are delayed or advanced, respectively).

A significant civil application of GPS is aeronautical navigation, especially during the approach and landing phases of aircrafts. To mitigate the ionospheric effects during these procedures, GNSS Augmentation Systems are being developed to improve the navigation system requirements on accuracy, reliability, integrity and availability (ICAO, 2001). That is, the absolute positioning provided by GNSS alone does not meet basic requirements that ensure the safety of aircrafts and therefore of users. GNSS Augmentation Systems are designed to perform and process measurements, and to transmit correction messages to aircrafts, which will use them to improve their position estimates.

The most important augmentation systems are categorized as "groundbased" or "space-based", depending on the coverage area and how they transmit correction messages. Ground-Based Augmentation Systems (GBAS) are designed to provide service in a local area (within several kilometers to tens of kilometers from airports) and to transmit correction messages to aircrafts through ground stations. The GBAS includes two or more GNSS receivers, which collect and 
process pseudorange, carrier-phase and Doppler measurements. This network of ground receivers is located at precisely known locations and the central monitor continually updates its error estimates for each monitored satellite and transmit the most recent correction messages (Kaplan and Hegarty, 1996).

Recently, several GBAS have been installed in many airports in the United States of America (USA), United Kingdom (UK), Germany, and other countries. In the future, GBAS is expected to become one of the main take-off and landing systems for airports.

In Brazil, the Departamento de Controle do Espaço Aéreo (DECEA) performed initial assessments of the operation of a Space-Based Augmentation System (SBAS) for navigation and aircraft approaches. A SBAS is designed to provide service over a wide area (typically, over a country) and to transmit correction messages to aircrafts through geostationary satellites. The investigation carried out at the time demonstrated non-compliance with the safety requirements for use in the Brazilian region. For this reason, DECEA chose to invest in the GBAS system (ICEA, 2013).

In 2011, DECEA acquired a GBAS station SLS-4000 from the North American company Honeywell, which was installed at the Tom Jobim International Airport (Galeão) in Rio de Janeiro, Brazil, to evaluate the quality and security of air navigation service in Brazil. The equipment is only certified for operation in the territories of the USA, Germany and part of Australia, through risk models developed for each country (ICEA, 2013).

Note that ionospheric disturbances that are frequently observed in the lowlatitude and equatorial regions, including Brazil and India (Basu et al., 1981; Aarons, 1982; Woodman and La Hoz, 1976; de Paula et al., 2007; Galera Monico et al., 2014; Moraes et al., 2018), potentially limiting to radionavigation by GNSS, are not usually observed in the mid-latitude region including the USA. Studies are necessaries to model, simulate and analyze the ionospheric effects characteristic of the Brazilian region in the context of GBAS. This is the motivation for this research. 
There are previous studies on the above effects based on the estimation of ionospheric gradients using spatial and time methods for days with high geomagnetic activity (Datta-Barua et al., 2006; Pullen et al. 1998; Datta-Barua, 2008; Pereira, 2018). These authors designed a set of algorithms for GBAS that bound the error of differentially corrected measurements and position estimations based on ionospheric gradients (Xie, 2004; Lee, 2005).

Ionospheric scintillation (random fluctuations of the amplitude and phase of transionospheric signal), which may occur during the evenings in low-latitude and equatorial regions, adversely affects GPS transmissions. Severe cases of ionospheric scintillation may even cause losses of phase lock in GPS receivers. The physics of ionospheric scintillation has been studied for several decades (Singleton et al., 1961; Crane, 1977) and their effects on GPS signals are summarized in (Kintner et al., 2007; Beniguel et al., 2007; Rino, 2011; Carrano et al., 2005). In the present context, it is important to highlight the statistical modeling and evaluation of the impact of ionospheric amplitude scintillation on the performance of GPS receivers for equatorial and low latitudes (Moraes, 2013), as well as the characteristics of GPS signal fades due to scintillation from the perspective of GPS-assisted navigation (Conker et al., 2003; Seo, 2010).

\subsection{Research Objectives}

The main objective of this thesis is to model, estimate and analyze the effects of the ionospheric events that affect the GBAS operation (generation of corrections for aircraft during landing phase) in the low-latitude and equatorial regions.

To achieve the above general objective, it is necessary to identify and reach specific objectives that include:

- Development of a computational program to estimate the vertical Total Electron Content (vTEC) using measurements from GPS receivers of the Rede Brasileira de Monitoramento Contínuo, operated by the Instituto Brasileiro de Geografia e Estatística (RBMC/IBGE); 
- Use of the results from the above activity to statistically model the differences (residuals) between vTEC values obtained by the above measurements and the International Reference Ionosphere (IRI 2016) model, considering solar and geomagnetic activities, as well as season, local time, and geomagnetic latitude;

- Estimation and analysis of time and spatial ionospheric gradients in the Brazilian region during years with high and low solar and geomagnetic activity;

- Statistical analysis and comparison of ionospheric scintillation in the Brazilian region;

- Use of the statistical results of vTEC, in combination with a ionospheric scintillation, tropospheric, multipath, and other models, to generate GPS signals-in-space represented by pseudorange, carrier phase and received signal models that will be detected by the GBAS reference stations and the aircraft receiver;

- Develop a GBAS simulation model to process the received signals in space and estimate pseudorange corrections;

- Combine the GPS signals-in-space and the GBAS simulation models to estimate aircraft position errors (before and after corrections), as well as the availability and integrity of the measurements and protection levels for safety landing.

\subsection{Description of the Following Chapters}

Since major contributions will be thoroughly described in this thesis, only a brief summary of these efforts is outlined below.

The second chapter describes in more detail the fundamental concepts and the architecture of GPS and a brief description of a GBAS, together with the requirements for aeronautical navigation and some effects that affect the precise positioning of the aircraft.

The third chapter describes the ionospheric effects and the methodology to estimate the VTEC using GPS observations. This chapter also present estimations 
of VTEC using the IRI prediction model and performs a statistical comparison between the empirical (provided by RBMC/IBGE measurements) and the IRI model, considering solar and geomagnetic activities, as well as season, local time, and geomagnetic latitude.

The fourth chapter presents a quantitative and statistical analysis of ionospheric GPS vTEC and gradient estimations using the Time-Step and StationPair methods based on data from the RBMC/IBGE.

Next, the fifth chapter describes a statistical model of a GPS signals-inspace model, which considers IRI outputs and residual from RBMC/IBGE estimates, in combination with ionospheric scintillation, tropospheric delay, multipath, and other modules.

The sixth chapter describes and specifies the GBAS ground facility model, which tests the GPS signal-in-space and creates correction messages of pseudoranges for aircrafts during their approach to airports under ionospheric events in equatorial and low latitude regions.

Chapter seven presents and discuss results from the test monitors and corrections carried out by the GBAS model in scenarios of interest. Whenever possible and applicable, the results are compared with those available in the literature.

Finally, the eighth chapter summarizes the most important results obtained by this Thesis and suggests future works. 


\section{Global Positioning System (GPS) and Augmentation Systems}

\subsection{GPS}

The first navigation and positioning satellite system was the NAVigation Satellite with Time and Ranging (NAVSTAR) Global Positioning System (GPS). It is operated by the government of the United States of America. Developed in the early 1970s for military purposes by the Department of Defense (DoD), it was later made available to civilians. In a letter to ICAO in 1994, the United States offered the GPS Standard Positioning Service to support the needs of civil aviation users free of charge (CANSO, 2005).

GPS is composed of space, control and user segments. Currently, the space segment of GPS consists of a large number of satellites (32), orbiting the Earth in 6 almost-circular orbits at an inclination of approximately $55^{\circ}$ (tilt relative to the Earth's equatorial plane) at an altitude of about 20,180 km as shown in Figure 2.1. A satellite takes a little less than 11 hours and 58 minutes to complete its orbit. From a fixed location on Earth, it is visible for the maximum of approximately five hours, provided no obstacles are present. The GPS satellites transmit L-band signals in three frequencies $\left(\mathrm{f}_{\mathrm{L} 1}=1575.42 \mathrm{MHz}, \mathrm{f}_{\mathrm{L} 2}=1227.60 \mathrm{MHz}\right.$, and $\mathrm{f}_{\mathrm{L} 5}=$ $1176.45 \mathrm{MHz}$ ). Only 12 satellites of the IIF block actually emit the three frequencies.

The $\mathrm{L}_{1}$ carrier phase is modulated by Pseudo Random Noise (PRN) codes known as the Coarse/Acquisition code (C/A code), and by the Precise or Protected Code ( $\mathrm{P}$ code). The $\mathrm{L}_{2}$ carrier is modulated by the $\mathrm{P}$ code and by the civil code L2C. The L5 signal is modulated by a transmission rate ten times higher than the C/A code - this signal is protected worldwide for the use of aeronautical radionavigation and to support aviation security applications. 


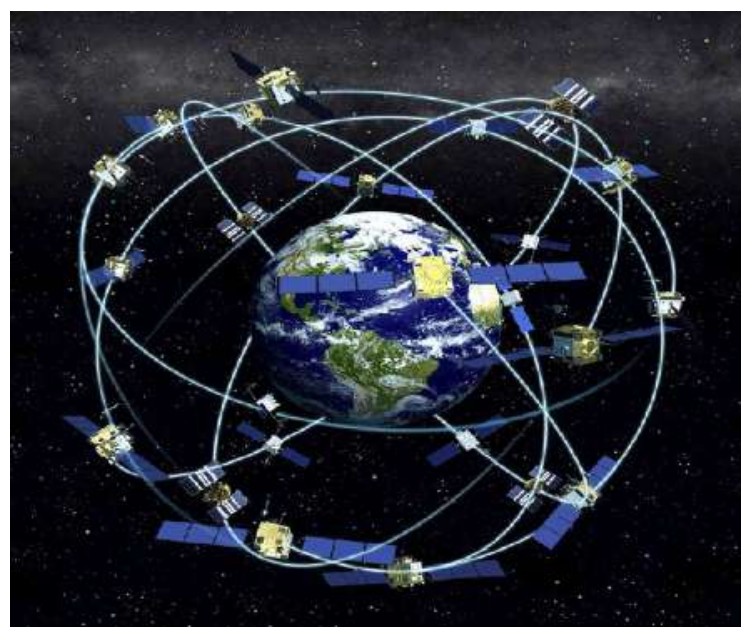

Figure 2.1: GPS satellite network.

[reproduced from www.space.com]

The control segment of the GPS consists of a master control station, monitor stations and ground antennas. The main responsibilities of the control segment are to monitor and control the satellites, determine and predict the satellite orbits and clock behaviors, and periodically upload navigation messages to the satellites (Hofmann-Wellenhof et al., 2008).

The user segment includes antennas and receivers to acquire and process the satellite signals as GPS observables. The observations provided by a GPS receiver yield carrier phase delays $L$, pseudoranges $P$ for the frequencies ( $f_{L 1}, f_{L 2}$ and $f_{L 5}$ ), Doppler and Signal-to-Noise Ratio (SNR). The GPS observables for each receiver are provided in the internal format of the receiver, which makes processing data of different receiver types difficult. To overcome this difficulty, a standard data format was developed: the Receiver Independent Exchange Format (RINEX).

The time system adopted by GPS is the Universal Coordinated Time (UTC), without the introduction of the leap second corrections (Monico, 2008). The official GPS spatial reference system is the World Geodetic System of 1984 (WGS84). All the satellite and user positions are stated and calculated in WGS84 coordinates. WGS84 is a 3D Earth-Centered Earth-Fixed coordinate system with its origin at the Earth's center of mass and is defined as follows, according to Figure 2.2 (Eurocontrol, 1998). 


$\begin{array}{ll}x^{\text {WGS84 }} & \text { equatorial axis pointing towards the Prime (Greenwich) Meridian } \\ z^{\text {WGS84 }} & \text { pointing towards the terrestrial motion pole } \\ y^{\text {WGS84 }} & \text { equatorial axis composing a right-handed orthogonal coordinate system }\end{array}$

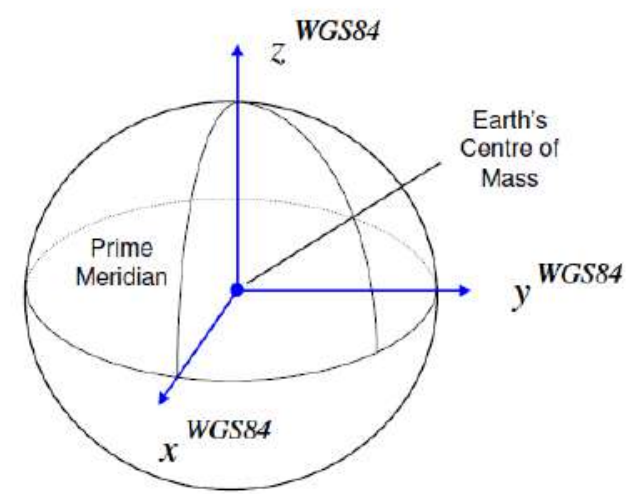

Figure 2.2: WGS84 Coordinate System.

\subsubsection{GPS Observable Error Sources}

The GPS measurements are subject to multiples error sources, which reduce the accuracy of GPS positioning. These error sources can be organized into three categories, as satellite related, receiver related, and signal propagation errors. The satellite-related errors are orbital errors, satellite clock errors and frequencydependent delays due to the satellite hardware. The receiver-related errors consist of receiver clock errors, receiver hardware delays and measurement noise. The signal propagation errors include ionospheric and tropospheric delays, as well as multipath. These error sources are briefly reviewed below.

\section{Orbital Error}

The position and the velocity information for the GPS satellites can be determined by broadcast ephemerides (orbits) or precise ephemerides. The broadcast ephemerides are computed and uploaded to the GPS satellites by the master station of the control segment, depending on observations at the monitor stations. The orbital information is broadcast in real time and is part of the navigation message in the form of Keplerian parameters. These orbital data could be accurate to approximately $1 \mathrm{~m}$ (Hofmann-Wellenhof et al., 2008). 
The precise ephemerides contain satellite positions and velocities with intervals of 15 minutes, which are provided by the International GNSS Service (IGS). There are several types of precise orbit data depending on the delay for their availability. The IGS Final Orbits are the most accurate orbital information, which are made available 13 days after the observations. Slightly less accurate ephemerides are provided as IGS Rapid Orbits and IGS Ultra Rapid Orbits with delays of 17 hours and 3 hours, respectively. The accuracy of the precise ephemerides is $5 \mathrm{~cm}$ or even better. The precise ephemerides are provided in files of Standard Product 3 (SP3) format (Dach et al., 2007).

Actually, the IGS is working to ensure the availability of Real Time Service (RTS) for scientific, educational, and commercial applications. Through the RTS, the IGS extends its capability to support applications requiring real-time access to IGS products. RTS is a GNSS orbit and clock correction service that enables precise point positioning (PPP) and related applications.

\section{Clock Errors}

The GPS system uses GPS time as its time scale. GPS time is an atomic time scale and is referenced to Universal Time Coordinated (UTC). Clock errors in GPS observables are due to the deviations of satellite and receiver oscillators from GPS time.

The GPS satellites are equipped with cesium or rubidium oscillators. These atomic clocks are highly accurate and stable. However, the satellite clock errors, typically less than $1 \mathrm{~ms}$, are still large enough to require correction. The deviation of each satellite clock from GPS time is estimated, modeled and broadcast as a component of the navigation message by the control segment. After the corrections applied, the residual satellite clock errors are typically less than a few nanoseconds (Mohinder et al., 2007).

In general, receivers use quartz crystal oscillators. Although receiver clock errors are much higher as compared to satellite clock errors, they are estimated with the receiver position or eliminated by differencing approaches. In GPS positioning, receiver clock errors are considered systematic errors that can be reduced by differencing the GPS code and phase observables for each epoch. 


\section{Hardware delays}

Delays in hardware of satellites and receivers result in frequency-dependent biases on both pseudorange and carrier phase measurements. These biases are not absolute values. Hence, they are not generally included in observation equations and modeled with clock errors. However, they should be taken into account for the combinations of observations in some situations; e.g. geometry linear combination for ionosphere modeling (Dach et al., 2007).

\section{Ionospheric delays}

The ionosphere is the layer of atmosphere between approximately $60 \mathrm{~km}$ and $1000 \mathrm{~km}$ above the Earth that contains electrically charged particles (electrons and ions). These particles delay the satellite signals and can cause a significant amount of satellite position error.

Ionospheric delay varies with solar activity, time of year, season, time of day and location. This makes it very difficult to predict how much ionospheric delay is influencing the calculated position. Ionospheric delay also varies based on the radio frequency of the signal passing through the ionosphere. GNSS receivers that can receive more than one GNSS signal (L1 and L2, for example) can use this characteristic to their advantage. By comparing the L1 and L2 measurements, the receiver can determine the amount of ionospheric delay and remove the influence of the error from the calculated position. For receivers that can only track a single GNSS frequency, ionospheric models are used to reduce ionospheric delay errors. Due to the varying nature of ionospheric delay, models are not as effective as using multiple frequencies at removing this source of errors.

\section{Tropospheric delays}

The troposphere is the layer of atmosphere closest to the surface of the Earth. Variations in tropospheric delay are caused by the changing humidity, temperature and atmospheric pressure in the troposphere.

Since tropospheric conditions are very similar within a local area, the base station and rover receivers experience very similar tropospheric delay, always 
considering the height of the receivers. GNSS receivers can also use tropospheric models to estimate the amount of error caused by tropospheric delay.

\section{Multipath}

Multipath is the arrival of a signal at the GPS receiver antenna via two or more different paths. It is usually due to the reflection or scattering of the signal from surfaces such as the ground, buildings, streets, and vehicles. Multipath affects both code and carrier phase measurements in a GPS receiver. The effect on P-code measurements can reach decimeters to meters, while the range error on $\mathrm{C} / \mathrm{A}$ code measurements is on the order of several meters. The maximum error due to multipath is about $5 \mathrm{~cm}$ for carrier phase observations. Multipath can be eliminated or reduced by selection of the site locations to avoid reflections, using designed antennas, utilizing absorbing materials near the antenna, and employing receivers with related software to detect their effects (Seeber, 2003).

\subsection{Air Navigation Using GPS}

A significant civil application of GPS is the support of air navigation. With the growing global demand for air travel in the 21 st century, aviation is being supported by some benefits that GPS can provide, like radionavigation, economy, and security of flight operations.

Air navigation, especially during the approach and landing phases of aircrafts, requires better accuracy than absolute positioning can provide (Lee, 2005). In this context, the use of Differential GPS (DGPS) significantly improves the requirement of air operations (Alves et al., 2011; Hofmann-Wellenhof et al., 2008; Monico, 2008). Differential positioning is used to improve user position estimations by applying corrections from continuously monitored GPS station, known as reference or base stations (with precisely known coordinates).

The basic principle for DGPS feasibility is the consideration of a spatial and time correlation of ionospheric, tropospheric, ephemeris, and satellite clock errors between the reference station and the user (mobile station), provided that the distance between both has the maximum order of a few tens of kilometers (Lee, 2005), as shown in Figure 2.3. 


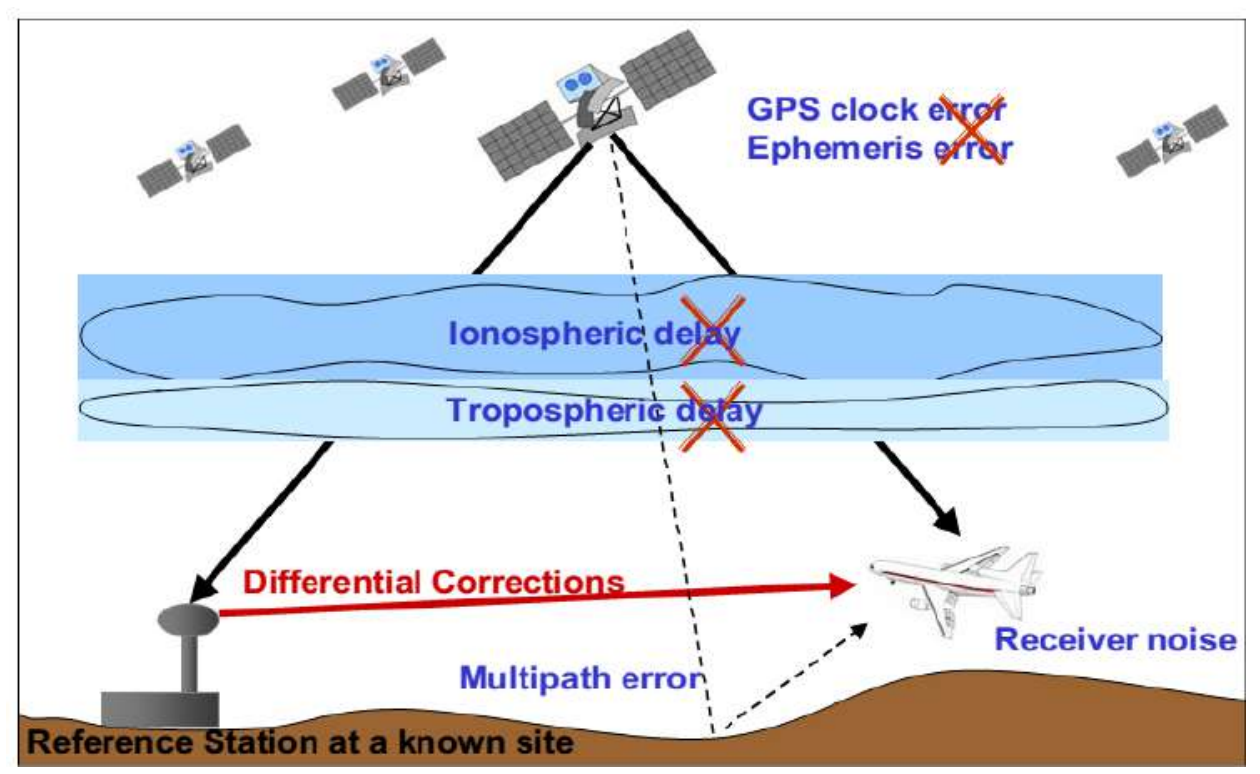

Figure 2.3: Differential GPS.

[Adapted from Lee, 2005]

\subsection{Augmentation Systems}

Even though the GPS constellation and receivers meet some en-route and non-precision approach (NPA) requirements, they cannot be used as a single navigation method for more demanding precision procedures. To meet stricter requirements, the GPS navigation service is provided using combinations of various elements installed on the ground, on satellites and/or on board aircraft. These set of combinations are called augmentation systems (ICAO, 2001).

\subsubsection{Satellite-Based Augmentation System (SBAS)}

The SBAS is a Wide Area DGPS (WADGPS) service for users within a continental coverage area, in general. Designed to improve air navigation using the GPS satellites (combined or not with other constellations), the SBAS provides additional information through geostationary satellites. Among the information provided, the most important transmitted parameters are ionospheric delays, as well as satellite clock and ephemeris correction (Hoffman-Wellenhof et al., 2008). SBAS works by monitoring signals from GNSS satellites with the aid of ground reference stations, calculating corrections, and uploading them to a satellite in geostationary orbit for broadcast to aircraft and other users, as shown in Figure 2.4 . 


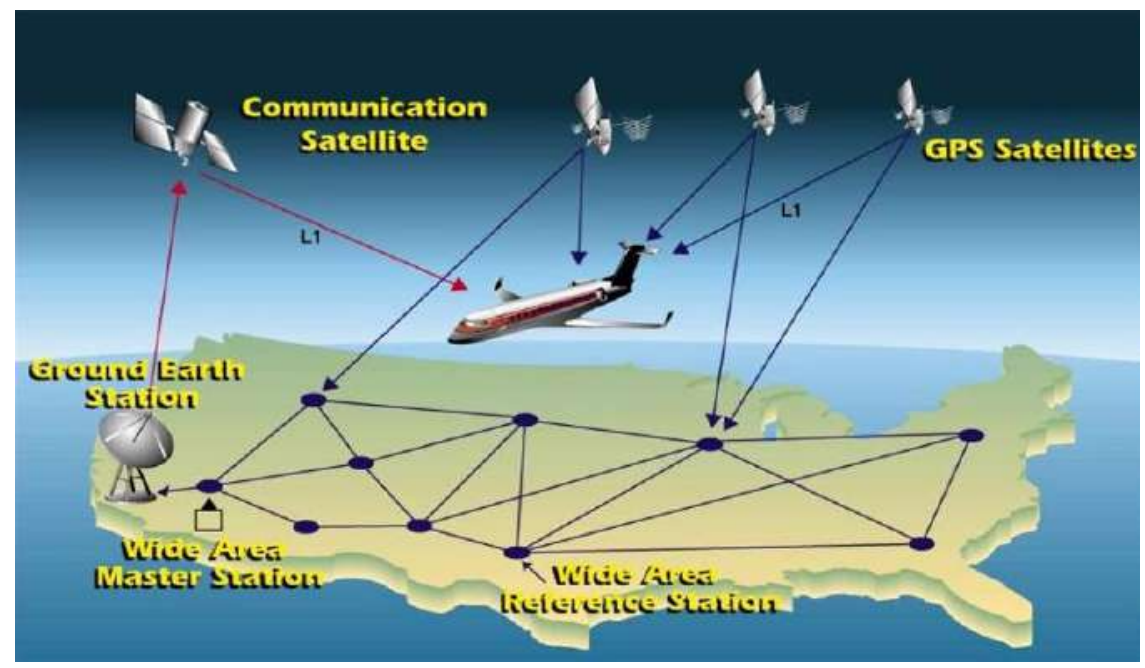

Figure 2.4: SBAS network. [Adapted from FAA, 2018]

SBAS systems are intended to support operations with less stringent requirements, as En-route, Terminal, Departure and Non-Precision Approach operations, and may meet the requirements for Category-I (CAT-I) Precision Approaches in less-demanding environments.

\subsubsection{Ground-Based Augmentation System (GBAS)}

GBAS was developed to be used in precision approaches, in principle for CAT-I, but with an intention of also reaching CAT-II and CAT-III. The precision approaches are performed by aircrafts equipped with a GPS receiver.

The principle of GBAS systems are based on Differential Corrections. The main idea of Differential Corrections is illustrated in Figure 2.5. The observable corrections are determined using a set of GPS reference receivers (generally four) equipped with simple frequency receivers (L1) that computes and broadcasts differential corrections and integrity-related information for them, based on its own surveyed position. Thus, they reduce systematic errors and aim at guaranteeing the requirements for CAT-I operation. These differential corrections are transmitted to aircrafts from the ground system via a Very High Frequency (VHF) Data Broadcast (VDB) link. The broadcast information includes pseudorange corrections, integrity parameters and various locally relevant data, such as Final Approach Segment (FAS) data, referenced to the World Geodetic System (WGS-84). 


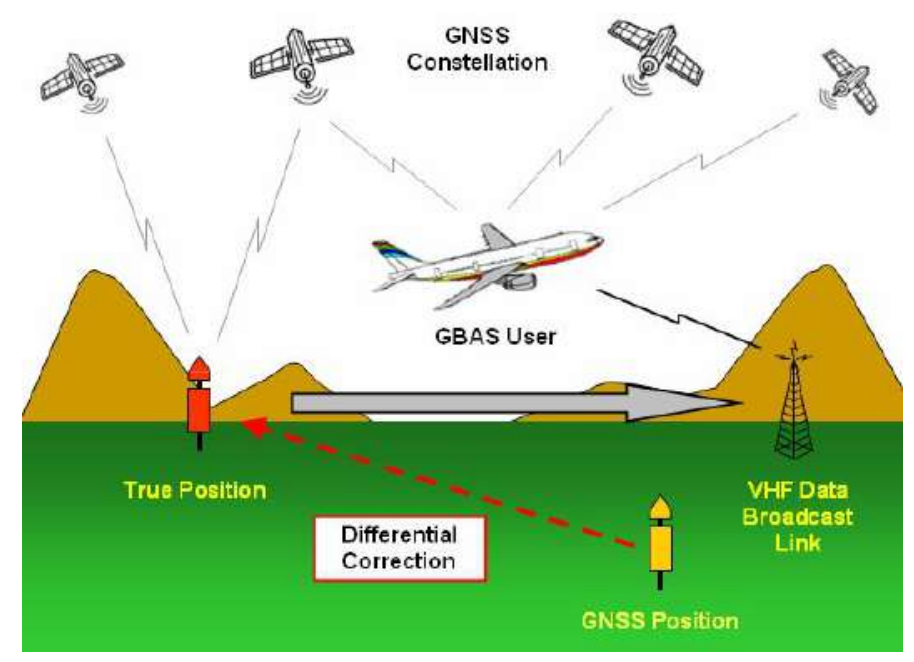

Figure 2.5: Differential correction of GBAS.

[Adapted from Ferreira, 2007]

Together with differential corrections, GBAS systems will also provide users with integrity data of real position. This type of information is crucial in safety-of-life operations, such as civil aviation, and allows users to evaluate the reliability of the information given by the GNSS system, by providing high levels of confidence bounds for the positioning errors.

Next, this chapter briefly introduces the GBAS architecture in Figure 2.6. The complete augmentation system will meet requirements with respect to accuracy, integrity, continuity, and availability. Chapter 5 will describe how the Signal-in-Space are simulated. Chapter 6 will describe and specify how these signals are processed to generate differential corrections, and how the residual errors are characterized by a GBAS. 


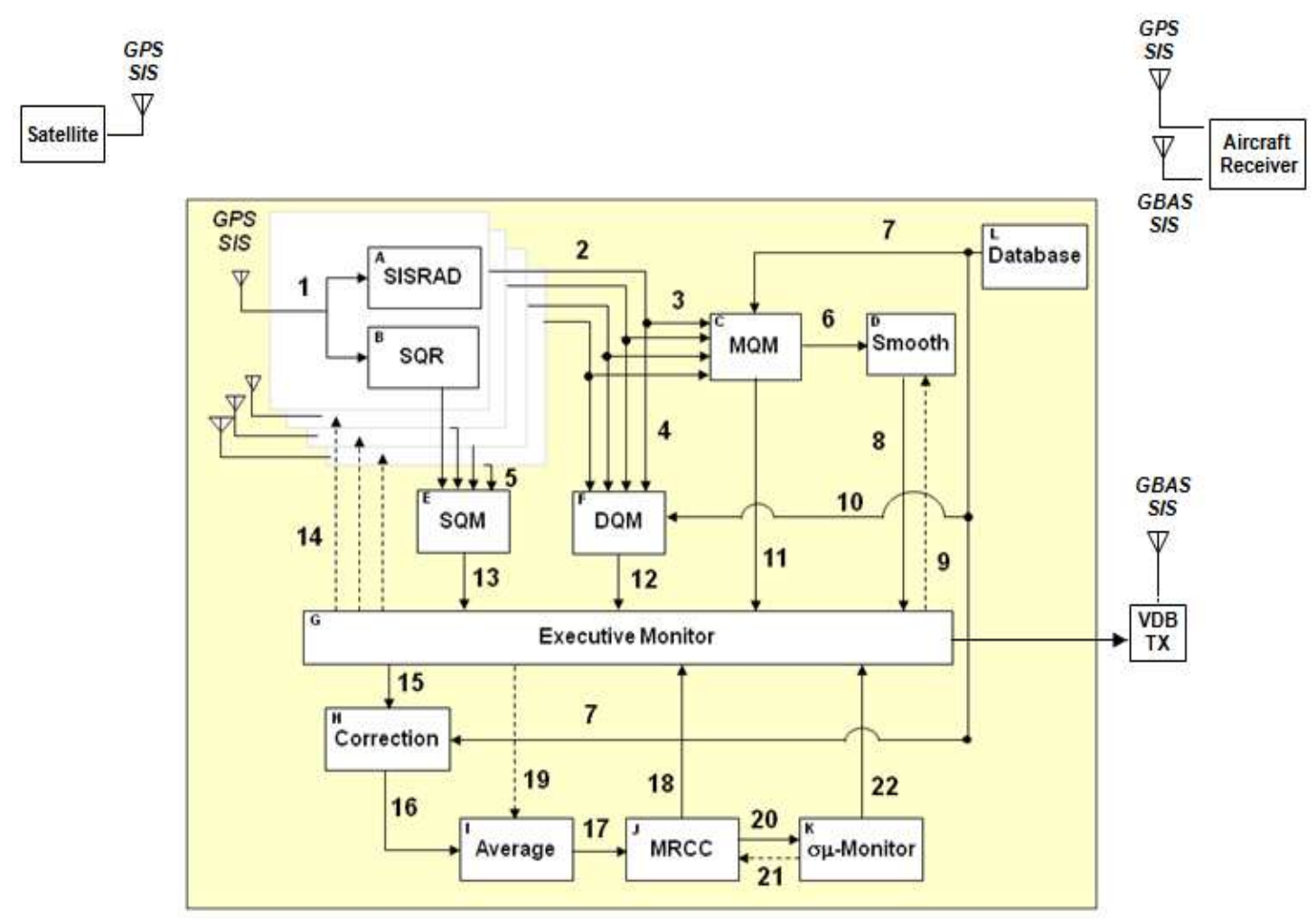

Figure 2.6: Block Diagram of the GBAS Ground Facility (GGF) model. [Adapted from LAAS KTA Group, 1998]

Figure 2.6 shows the GBAS Ground Facility model functional block diagram. The Signal-in-Space Receiver and Decode (SISRAD) function provides pseudorange and carrier-phase measurements, together with received signal levels and navigation data messages that are the core input of the GBAS processing, which enables the generation of carrier-smoothed code differential corrections. The resulting GBAS corrections are used to derive improved user position solutions. The GGF model is responsible for generating and broadcasting corrections, as well as for detecting and alerting a wide range of possible failures in the GPS Signal-in-Space (SIS) or in the GGF model itself. In this regard, the Integrity Monitor Testbed (IMT) processing utilizes different types of monitoring algorithms, to isolate and remove error sources (some of which may trigger more than one monitoring algorithm). Chapter 6 will explain this block diagram in more detail.

The navigation system is characterized in terms of accuracy, integrity, availability and continuity: 


\section{Accuracy}

The accuracy of a system is the degree of closeness of measurements to that quantity of the true value. In air navigation, accuracy is a measure of the difference between the estimated position and the true aircraft position under nominal fault-free conditions. It is typically a $95 \%$ bound on the Navigation System Error (NSE), which represents the error in the estimation of the aircraft position.

\section{Integrity}

Integrity is a measure of the trust that can be placed in the correctness of the information supplied by the air navigation system. Integrity includes the ability of a system to provide timely and valid warnings to users (ICAO, 1999).

The previous description of integrity is a qualitative definition. A quantitative approach can be described by the Integrity Risk, which is defined by the probability that the navigation system provides information that, processed under fault-free conditions, results in an unacceptable NSE, without timely warning to the user. That is, the Integrity Risk is expressed by a probability and an exposure interval. Consequently, the Required Navigation Performance (RNP) Integrity parameter value will be the probability that an Integrity fault does not occur during a certain interval (BOEING, 2005; Ferreira, 2007).

From the Integrity Risk definition, two important parameters related to integrity are derived:

- Alert Limit is the maximum allowable NSE for a certain operation and defines that an unacceptable position error is dangerous. It is expressed in Horizontal and Vertical Alert Limits (HAL and VAL respectively), as shown in Figure 2.7.

- Time to Alert is the maximum time that the navigation system has to issue a warning to the user alerting for an integrity fault; it defines what constitutes a timely warning.

According to the definitions, to evaluate the integrity of navigation systems, the instantaneous NSE would have to be estimated and checked against the Alert 
Limits for the flight navigation in process. However, the NSE is not observable by the aircraft operator and another approach to evaluate the system Integrity has to be used. The standard approach estimates the worst-case NSE and compares this value with the corresponding Alert Limit values (to be presented in Chapter 6). These limits for NSE are known as Protection Levels and represent highconfidence bounds for the NSE for air navigation (ICAO, 2001).

Similarly, to the positioning errors, the Protection Levels are represented by Horizontal and Vertical components (HPL and VPL) and are shown in Figure 2.7. The horizontal plane is defined as locally tangent to the navigation system space reference, which in GPS is the WGS84 ellipsoid. The vertical axis is locally perpendicular to the same reference. For safety, as shown in Figure 2.7, one needs the protection levels to be always smaller than the alert limits corresponding to the current phase of flight. The computation of protection levels will be discussed in Chapter 6.

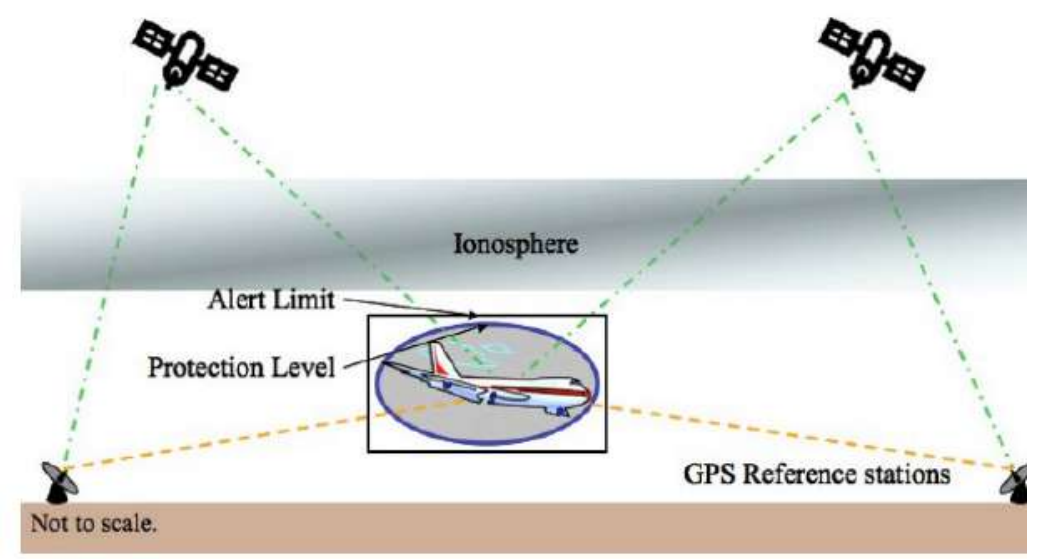

Figure 2.7: Alert limits and protections levels.

The current requirements and VAL/HAL - bounds to maximum tolerable VPL/HPL - for these precision approaches are summarized in Table 2.1, where the values representing integrity probabilities are expressed linearly, and not as percentages. For example, the integrity risk for CAT-I is $2 \times 10^{-7}$ for one approach, the duration for one approach is approximately is $150 \mathrm{~s}$, this value can be visualized as follows: when executing $5 \times 10^{6}$ approaches, it is allowed not to give a warning to the pilot more than one time that the parameters of the navigation system are out of tolerable. 
Table 2.1: Requirements for precision approach and landing.

[Adapted from Lee, 2005]

\begin{tabular}{|c|c|c|c|c|c|c|}
\hline \multirow{2}{*}{$\begin{array}{c}\text { Phase } \\
\text { of } \\
\text { Flight }\end{array}$} & \multirow{2}{*}{$\begin{array}{c}\text { Accuracy } \\
(95 \% \\
\text { error) }\end{array}$} & \multicolumn{2}{|c|}{ Integrity } & \multirow{2}{*}{$\begin{array}{l}\text { Alert } \\
\text { Limit }\end{array}$} & \multirow[b]{2}{*}{ Continuity } & \multirow[b]{2}{*}{ Availability } \\
\hline & & $\begin{array}{c}\text { Time } \\
\text { to alert }\end{array}$ & $\begin{array}{c}\text { Prob } \\
\text { (HMI) }\end{array}$ & & & \\
\hline LPV & $\begin{array}{l}\mathrm{H}: 16 \mathrm{~m} \\
\mathrm{~V}: 20 \mathrm{~m}\end{array}$ & $10 \mathrm{~s}$ & $\begin{array}{l}2 \times 10^{-7} \\
\text { /aproach }\end{array}$ & $\begin{array}{l}\mathrm{H}: 40 \mathrm{~m} \\
\mathrm{~V}: 50 \mathrm{~m}\end{array}$ & $\begin{array}{l}5.5 \times 10^{-5} \\
\text { /aproach }\end{array}$ & $\begin{array}{c}0.99 \text { to } \\
0.99999\end{array}$ \\
\hline APV-2 & $\begin{array}{l}\mathrm{H}: 16 \mathrm{~m} \\
\mathrm{~V}: 7.6 \mathrm{~m}\end{array}$ & $6 \mathrm{~s}$ & $\begin{array}{l}2 \times 10^{-7} \\
\text { /aproach }\end{array}$ & $\begin{array}{l}\mathrm{H}: 40 \mathrm{~m} \\
\mathrm{~V}: 20 \mathrm{~m}\end{array}$ & $\begin{array}{l}5.5 \times 10^{-5} \\
\text { /aproach }\end{array}$ & $\begin{array}{c}0.99 \text { to } \\
0.99999\end{array}$ \\
\hline CAT I & $\begin{array}{l}\mathrm{H}: 16 \mathrm{~m} \\
\mathrm{~V}: 4.0 \mathrm{~m} \\
\text { to } 7.6 \mathrm{~m}\end{array}$ & $6 s$ & $\begin{array}{l}2 \times 10^{-7} \\
\text { /aproach }\end{array}$ & $\begin{array}{l}\mathrm{H}: 40 \mathrm{~m} \\
\mathrm{~V}: 12 \mathrm{~m}\end{array}$ & $\begin{array}{l}5.5 \times 10^{-5} \\
\text { /aproach }\end{array}$ & $\begin{array}{c}0.99 \text { to } \\
0.99999\end{array}$ \\
\hline CAT II & $\begin{array}{l}\mathrm{H}: 6.9 \mathrm{~m} \\
\mathrm{~V}: 2.0 \mathrm{~m}\end{array}$ & $2 \mathrm{~s}$ & $\begin{array}{l}2 \times 10^{-9} \\
\text { /aproach }\end{array}$ & $\begin{array}{l}\mathrm{H}: 17.4 \mathrm{~m} \\
\mathrm{~V}: 5.3 \mathrm{~m}\end{array}$ & $\begin{array}{l}4 \times 10^{-6} \\
/ 15 \mathrm{~s}\end{array}$ & $\begin{array}{c}0.99 \text { to } \\
0.99999\end{array}$ \\
\hline $\begin{array}{c}\text { CAT } \\
\text { III }\end{array}$ & $\begin{array}{l}\mathrm{H}: 6.1 \mathrm{~m} \\
\mathrm{~V}: 2.0 \mathrm{~m}\end{array}$ & $\begin{array}{c}1 \mathrm{~s} \text { to } \\
2 \mathrm{~s}\end{array}$ & $\begin{array}{l}2 \times 10^{-9} \\
\text { /aproach }\end{array}$ & $\begin{array}{l}\mathrm{H}: 15.5 \mathrm{~m} \\
\mathrm{~V}: 5.3 \mathrm{~m}\end{array}$ & $\begin{array}{l}2 \times 10^{-6} \\
/ 15 s\end{array}$ & $\begin{array}{c}0.99 \text { to } \\
0.99999\end{array}$ \\
\hline
\end{tabular}

\section{Availability}

Availability is the probability (stated in Table 2.1) that the navigation system is operational during a specific flight operation; i.e., the Accuracy and Integrity provided by the system meet the requirements for the desired operation (ICAO, 1999). The navigation system is considered available for use in a specific flight operation if the Protection Levels estimated are inferior to the corresponding specified Alert Limits for that same operation.

\section{Continuity}

Continuity is the ability of the system to perform its function without unpredicted interruption during the intended operation. Values stated in Table 2.1 are the probability that the system provides Continuity of service during the corresponding interval (ICAO, 1999). Continuity is a measure of the quality of the service. The Continuity Risk is also defined by the probability of any detected, but unscheduled, interruption after the initiation of an operation. 


\section{Estimation and Analysis of TEC Using GPS Measurements and IRI Model}

\subsection{Ionosphere}

The ionosphere is the ionized layer of Earth's atmosphere. This layer results from the emission of a spectrum of radiation by the sun that ionizes neutral atoms or molecules (Hargreaves, 1992; Kelley, 2009), in combination with several other processes. The boundaries for this layer are not clearly defined. However, it is generally accepted that ionosphere begins at approximately $50 \mathrm{~km}$ above Earth surface, above the neutral atmosphere layer, and extends to $1000 \mathrm{~km}$ or more where the protonosphere layer starts.

The vertical structure of the ionosphere is generally divided into three layers represented as D, E and F (Figure 3.1). The D layer lies approximately between about $50 \mathrm{~km}$ and $80 \mathrm{~km}$. In this region, ions are mainly produced by the X-ray radiation. Due to the recombination of electrons and ions, this layer is not present at night. The E layer ranges in height from $80 \mathrm{~km}$ to $150 \mathrm{~km}$ above the Earth's surface. This region has a high electrical conductivity at high latitudes. The highest layer of the ionosphere is divided into F1 and F2 sublayers. The F1 layer also disappears at night. F2 layer is the densest part of the ionosphere and has a high concentration of electron in the approximate range $300 \mathrm{~km}$ to $400 \mathrm{~km}$ in altitude. The height of the peak of the electron density highly depends on the several geophysical parameters (latitude, longitude, local time, season, the solar cycle, etc.) (Davies, 1990; Kelley, 2009). 


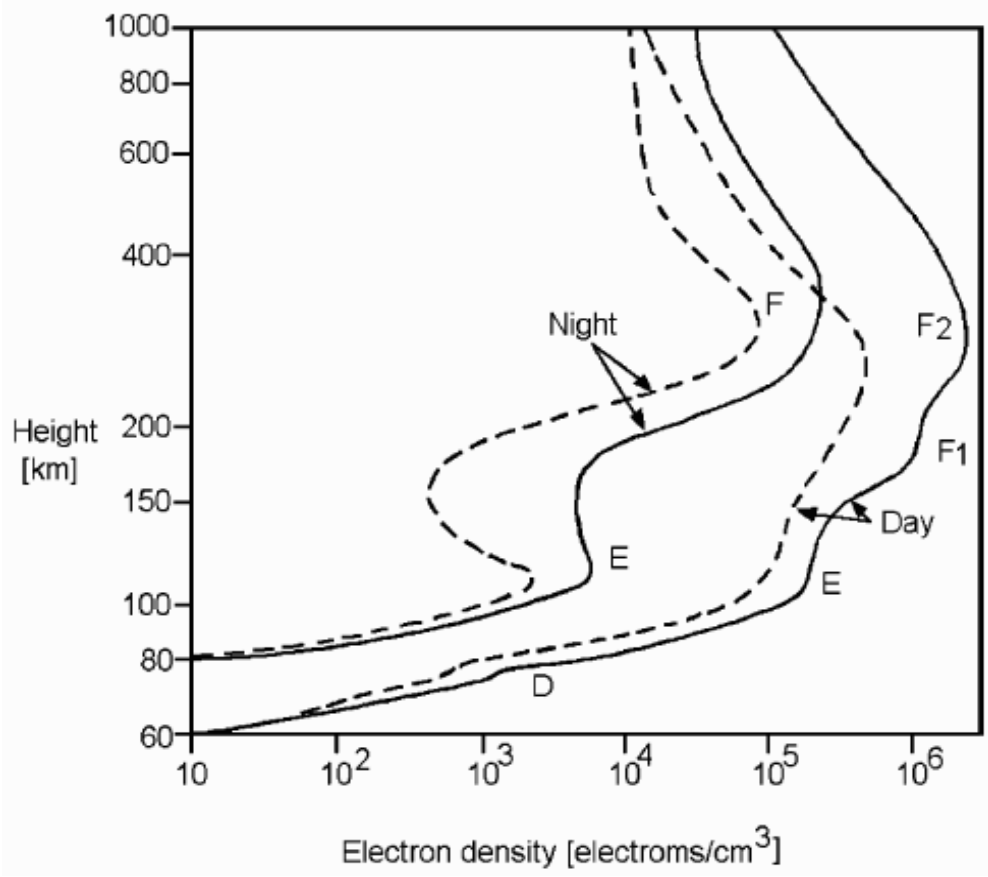

Figure 3.1: Vertical profile of the ionosphere.

[Reproduced from Hargreaves, 1992]

\subsubsection{Variations in the lonosphere}

The ionosphere presents spatial and time variations. In particular, spatial variations due to latitude result from electron interaction with the magnetic and electrical fields of the Earth. In association with the above parameter, the ionosphere can be divided into three geographical regions as shown in Figure 3.2a, with different behaviors: (1) the low-latitude or equatorial region, with geomagnetic latitudes in the interval from about $+30^{\circ}$ to $-30^{\circ}$, where peaks of electron concentration may occur (at the Equatorial Ionization Anomaly); (2) in mid-latitude regions (between about $\pm 30^{\circ}$ to $\pm 60^{\circ}$ of the geomagnetic latitudes), the variations in the ionosphere are more regular; (3) however, in the Polar or high latitudes, the variations, dominate by the geomagnetic field, are unpredictable (Seeber, 2003).

Since the solar radiation is the fundamental source of ionization, time variations in the ionosphere are connected to the activity of the sun. The electron concentration in the ionosphere undergoes variations on three time scales. One of the most important scales is related with the sunspot number, which are visibly patches over the surface of the sun. Sunspots are indicators that measure the intensity of the magnetic activity of the Sun. Figure $3.2 \mathrm{~b}$ shows the sunspot 
number variation between 1954 and 2018. As displayed in the Figure 3.2b, sunspot numbers follow a cycle of approximately 12 years. In addition to this 12 year cycle dependence, the electron density varies seasonally, due to the annual movement of the Earth around the sun. During the summer months, the electron density levels are typically higher than those in the winter. The third main scale of the time variation of the ionosphere results from the diurnal rotation of the Earth, having therefore a period of a solar day. Following the solar radiation with some delay, the concentration of electrons reaches its maximum values in the afternoon and has the minimum values before the midnight (Kleusberg and Teunissen, 1998).
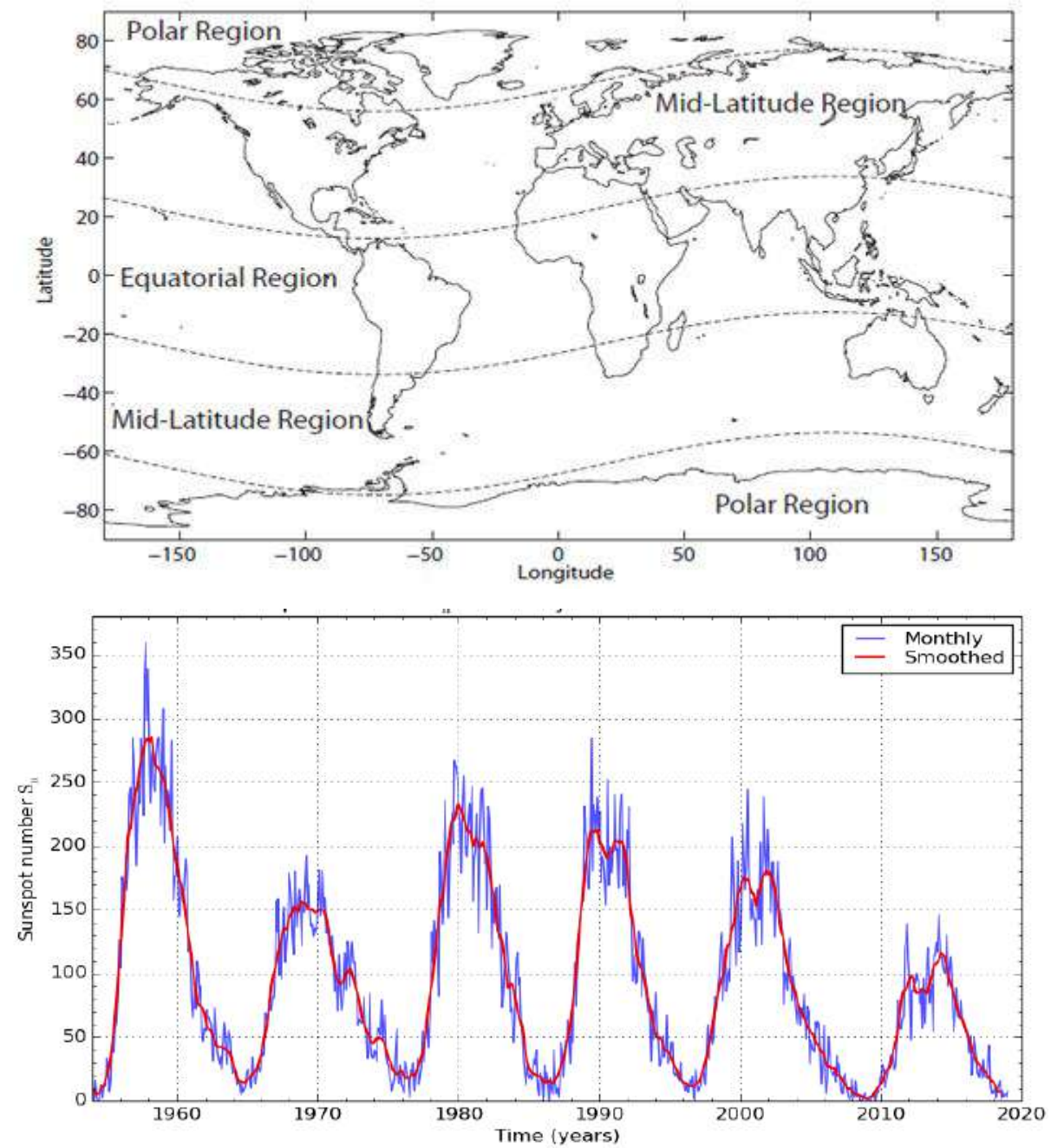

Figure 3.2: a) Ionospheric regions of the world; b) Sunspot number. [Reproduced from www.sidc.be/silso]

Beside these variations, the ionosphere is subjected to strong and unpredictable short-scale disturbances, which are called ionospheric irregularities. 
One class of ionospheric irregularities is associated with ionospheric storms, which are often coupled with severe disturbances in the magnetic field and strong solar eruptions (Schaer, 1999). Ionospheric storms could last from hours to several days and take place at global or hemispherical scales. In general, are initially and most intensely observed at high latitudes. Another important irregularity is the traveling ionospheric disturbances (TIDs), which are wave-like irregularities. They are thought to be related with perturbations of the neutral atmosphere and could be classified according to their horizontal wavelengths, speeds and periods (Davies, 1990).

The larger fraction of solar energy is absorbed within $\pm 30^{\circ}$ latitude zone centered on the equator (Abdu, 2005), so it is expected to have larger ionization at the region. However, one of the most prominent features in the ionosphere, known as equatorial ionization anomaly (EIA, also called the Appleton anomaly), occurs at the low latitudes, given origin to a depletion at equatorial latitudes and two ionization crests at low latitudes as explained below. The EIA is the occurrence of a trough in the ionization in the $F_{2}$ layer at the equator and crests at about $\pm 20^{\circ}$ in magnetic latitude. The Earth's magnetic field lines are horizontal at the magnetic equator. Solar heating and tidal oscillations in the lower ionosphere move plasma up and across the magnetic field lines. This sets up into the F layer, concentrating up into the $\mathrm{F}$ layer and subsequent diffusion along the magnetic lines, concentrating at \pm 20 degrees from the magnetic equator. This phenomenon is known as the equatorial fountain effect.

\subsubsection{Ionospheric Irregularities}

Irregularities in Earth's ionosphere can produce short-term signal variations in amplitude and phase; this phenomenon is called scintillation (McNamara, 1994; Langley, 1998) and can cause excessive stress in GPS signal tracking, which can lead to loss of phase or code lock and, consequently, poor navigation performance. These scintillation effects mainly occur in a belt of \pm 30 degrees either side of Earth's geomagnetic equator, and in the polar auroral zones as shown in Figure 3.2a. A very high electron content only occurs in equatorial regions. 
The observation period of scintillation can start at 18:00 LT and continued until the next day at 06:00 LT. Scintillations at $\mathrm{GHz}$ frequencies are normally observed between sunset hours and local midnight, but cases of post-midnight scintillation have also been observed. Post-midnight scintillations are usually associated with spread F triggered by storm-time disturbance electric fields (Aarons, 1982, Kintner et al., 2007). Scintillation effects are less significant from April through August in the American, African, and Indian longitude regions, but maximize in the Pacific region. From September through March the situation is reversed.

\subsection{Ionospheric Refraction}

In terms of geodetic positioning, the ionospheric parameter that produces an important part of the effects in GNSS signals is the total number of electrons measured along paths between Global Positioning System (GPS) satellites and monitoring stations. This parameter is usually called the Total Electron Content (TEC), described in Figure 3.3. The magnitude of the systematic error due to ionospheric refraction is directly proportional to the TEC in the ionospheric layer and inversely proportional to the square of the carrier wave frequency. In the course of this section this relationship between the TEC and the ionospheric refraction for phase and pseudorange measurements is developed.

By definition, the TEC is the number of electrons in a column with a crossed section of one square meter along a signal path from a GNSS satellite to a receiver antenna. The TEC can be expressed as (Seeber, 2003)

$$
T E C=\int_{\text {ray }} N_{e} d s\left[\frac{e l}{m^{2}}\right]
$$

where $N_{e}$ is the electron density $\left[\mathrm{el} / \mathrm{m}^{3}\right]$. Considering the simplified expression for its index of refraction (for relatively high frequencies), the ionospheric delay $I$ as function of the TEC can be expressed as

$$
I=\frac{K}{2 f^{2}} T E C
$$


where $K=80.62\left[\mathrm{~m}^{2} / \mathrm{s}^{2}\right]$ represents the ionospheric refraction and $f$ is the frequency of the system.

Equation (3.2) represents the major part of the ionospheric effect (Seeber, 2003). This is the first order of the total ionospheric delay caused by ionosphere disturbances. The higher order magnitude errors due to ionospheric effects can achieve a few centimeters and they can be relevant for high accuracy applications.

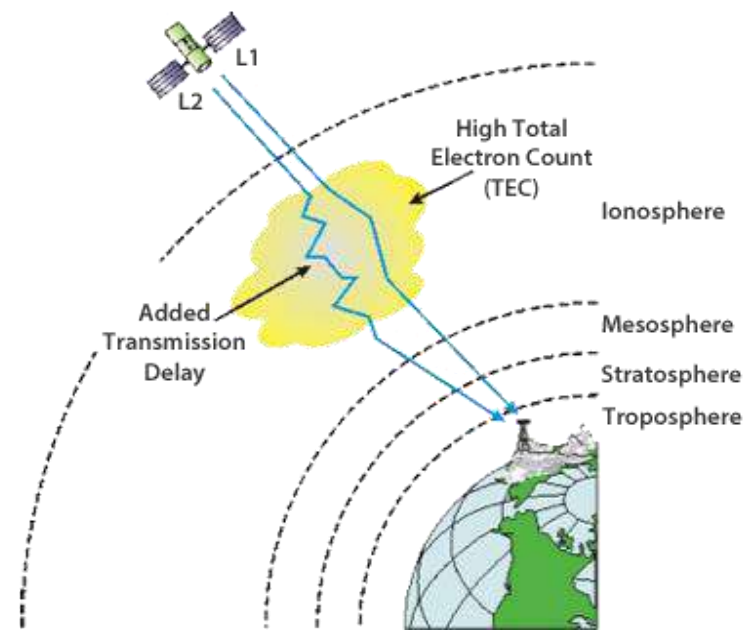

Figure 3.3: Effects of ionospheric refraction on GPS signals at different frequencies. [Adapted from http://www.reflexions.uliege.be]

\subsection{Extraction of Absolute TEC from GPS Observations}

The basic observables of GPS are the pseudorange $P$ and the carrier phase L. A less used third observable is the Doppler measurement, which represents the difference between the nominal and received frequencies of the signal. Using the difference between the pseudoranges $\left(P_{1}\right.$ and $\left.P_{2}\right)$ and the carrier phase measurements $\left(L_{1}\right.$ and $\left.L_{2}\right)$ at two frequencies, it is possible to compute the value of the slant TEC $\left(\mathrm{TEC}_{\mathrm{s}}\right)$ measured along a straight path from the satellite to the receiver (Hofmann-Wellenhof, 2008).

\subsubsection{TEC from Pseudorange Observation}

The GPS receivers use the C/A and P codes to determine the pseudorange, which is a measure of the distance between the satellite and the receiver, considering the effects from the medium. The receiver replicates the code 
generated by the satellite and determines the elapsed time for the propagation of the signal from the satellite to the receiver by correlating the transmitted code and the code replica. The pseudorange is computed by simply multiplying the time offset by the speed of light.

The pseudorange observation equations can be expressed as (Ciraolo et al., 2007, Carrano et al., 2009)

$$
\begin{aligned}
& P_{1}=\rho+c \cdot\left(\Delta t_{r}-\Delta t_{s}\right)+I_{1}+T+b_{1 r}^{P}+b_{1 s}^{P}+m_{1}+\varepsilon_{1}[\mathrm{~m}] \\
& P_{2}=\rho+c \cdot\left(\Delta t_{r}-\Delta t_{s}\right)+I_{2}+T+b_{2 r}^{P}+b_{2 s}^{P}+m_{2}+\varepsilon_{2}[\mathrm{~m}]
\end{aligned}
$$

where $P_{1}$ and $P_{2}$ are the pseudorange observations corresponding to frequencies $f_{1}$ and $f_{2} ; \rho$ is the geometric distance from the GPS receiver's antenna phase center at the epoch of signal reception to the GPS satellite's antenna phase at the epoch of the signal transmission; $c$ is the speed of the light; $\Delta t_{r}$ and $\Delta t_{s}$ are the receiver and satellite clock errors; $I_{1}$ and $I_{2}$ are the ionospheric delays for frequencies $f_{1}$ and $f_{2} ; T$ is the tropospheric delay, $b_{1 r}^{P}$ and $b_{2 r}^{P}$ are the instrumental biases for the receiver; $b_{1 s}^{P}$ and $b_{2 s}^{P}$ are the instrumental biases for the satellite, $m_{1}$ and $m_{2}$ are the associated with multipath effects; and $\varepsilon_{1}$ and $\varepsilon_{2}$ are thermal noise components. The indices 1 and 2 represent the frequencies $f_{1}$ and $f_{2}$, respectively.

Using equations (3.3) and (3.4) to form the difference $P_{2}-P_{1}$, neglecting the multipath and thermal noise terms, and considering that the geometric range, clock error, and tropospheric delay terms cancel, one gets

$$
\begin{gathered}
P_{2}-P_{1}=I_{2}-I_{1}+\left(b_{2 r}^{P}-b_{1 r}^{P}\right)+\left(b_{2 s}^{P}-b_{1 s}^{P}\right) \\
P_{2}-P_{1}=I_{2}-I_{1}+b_{r}^{P}+b_{s}^{P}
\end{gathered}
$$

Substituting the ionospheric delay represented in equation (3.2) into the pseudorange observation equation, it follows that 


$$
\begin{gathered}
P_{2}-P_{1}=\frac{K}{2}\left(\frac{1}{f_{2}^{2}}-\frac{1}{f_{1}^{2}}\right) T E C_{s l p}+b_{r}^{P}+b_{s}^{P} \\
T E C_{s l p}=\frac{2\left(f_{1} f_{2}\right)^{2}}{K\left(f_{1}^{2}-f_{2}^{2}\right)}\left(P_{2}-P_{1}\right)-b_{r}^{P}-b_{s}^{P}\left[\frac{e l}{m^{2}}\right]
\end{gathered}
$$

where $T E C_{s l p}$ is the slant TEC calculated using pseudorange measurements, $f_{1}$ and $f_{2}$ are the frequencies corresponding to GPS signals.

\subsubsection{TEC from Carrier Phase Measurement}

The carrier phase measurements correspond to the phase difference between the received signal (transmitted by the satellite) and the signal generated by the reference oscillator of the receiver. The equations for carrier phase measurements can be expressed as

$$
\begin{aligned}
& \phi_{1}=\rho+c \cdot\left(\Delta t_{r}-\Delta t_{s}\right)-I_{1}+T+b_{1 r}^{\phi}+b_{1 s}^{\phi}+\lambda_{1} N_{1}+m_{1}+\varepsilon_{1}[\mathrm{~m}] \\
& \phi_{2}=\rho+c \cdot\left(\Delta t_{r}-\Delta t_{s}\right)-I_{2}+T+b_{2 r}^{\phi}+b_{2 s}^{\phi}+\lambda_{2} N_{2}+m_{2}+\varepsilon_{2}[\mathrm{~m}]
\end{aligned}
$$

where $\phi_{1}$ and $\phi_{2}$ are the carrier phase observations corresponding to frequencies $f_{1}$ and $f_{2} ; \rho$ is the geometric range; $c$ is the speed of the light; $\Delta t_{r}$ and $\Delta t_{s}$ are the receiver and satellite clock errors; $I_{1}$ and $I_{2}$ is the ionospheric delay for frequencies $f_{1}$ and $f_{2} ; T$ is the tropospheric delay; $b_{1 r}^{\phi}$ and $b_{2 r}^{\phi}$ are the instrumental biases for the receiver; $b_{1 s}^{\phi}$ and $b_{2 s}^{\phi}$ are the instrumental biases for the satellite; $\lambda_{1}$ and $\lambda_{2}$ are the wavelength; $N_{1}$ and $N_{2}$ are the ambiguities; $m_{1}$ and $m_{2}$ are associated with multipath effects; and $\varepsilon_{1}$ and $\varepsilon_{2}$ are thermal noise components. The indices 1 and 2 represent the frequencies $f_{1}$ and $f_{2}$ respectively.

To use the carrier phase as an observable for positioning, the unknown number of cycles or ambiguity $N$, has to be determined by appropriate methods (Langley, 1998). The method used in this work will be describe below. 
Using equations (3.9) and (3.10) to form the difference $\phi_{1}-\phi_{2}$, neglecting the multipath and thermal noise terms, and considering that the geometric range, clock error, and tropospheric delay terms cancel, one gets

$$
\begin{gathered}
\phi_{1}-\phi_{2}=I_{2}-I_{1}+\left(b_{1 r}^{\phi}-b_{2 r}^{\phi}\right)+\left(b_{1 s}^{\phi}-b_{2 s}^{\phi}\right)+\left(\lambda_{1} N_{1}-\lambda_{2} N_{2}\right) \\
\phi_{1}-\phi_{2}=I_{2}-I_{1}+b_{r}^{\phi}+b_{s}^{\phi}+\left(\lambda_{1} N_{1}-\lambda_{2} N_{2}\right)
\end{gathered}
$$

Substituting the ionospheric delay represented by equation (3.2), $\phi_{1}=L_{1} \lambda_{1}$ and $\phi_{2}=L_{2} \lambda_{2}$ into the carrier-phase observation equation (3.12), it follows that

$$
\phi_{1}-\phi_{2}=\frac{K}{2}\left(\frac{1}{f_{2}^{2}}-\frac{1}{f_{1}^{2}}\right) T E C_{s l l}+b_{r}^{\phi}+b_{s}^{\phi}+\left(\lambda_{1} N_{1}-\lambda_{2} N_{2}\right)
$$

$$
T E C_{s l l}=\frac{2\left(f_{1} f_{2}\right)^{2}}{K\left(f_{1}^{2}-f_{2}^{2}\right)}\left(L_{1} \lambda_{1}-L_{2} \lambda_{2}\right)-b_{r}^{\phi}-b_{s}^{\phi}-\left(\lambda_{1} N_{1}-\lambda_{2} N_{2}\right)\left[\frac{e l}{m^{2}}\right]
$$

where $T E C_{s l l}$ is the slant TEC calculated using phase measurements, $f_{1}$ and $f_{2}$ are the frequencies corresponding to GPS signals and $L_{1}$ and $L_{2}$ are the number of cycles corresponding to the frequencies $f_{1}$ and $f_{2}$.

\subsubsection{Correction of Cycle Slips}

Cycle slips are abnormal jumps in carrier-phase measurements when the receiver phase tracking loops experience a temporary loss of lock or some other disturbing factor, which must be detected and corrected. Cycle slips can occur due to the failures in the receivers, as well as obstructions of the signal, high signal noise or low signal strength. The magnitude of a cycle slip may range from a few cycles to millions of cycles (Seeber, 2003).

In order to detect cycle slips, several testing quantities which are based on various combinations of GPS observations have been proposed (Seeber, 2003; Hofmann-Wellenhof et al., 2008). Some of these methods depend on the single, double or triple-differences of observations. Once a cycle slip is detected, it can 
be repaired using the former technique that requires an exact estimation of the size of the slip but could be done instantaneously.

Considering a stand-alone GPS receiver, the previous observation equations for carrier phase measurements can be reformulated as (Dai, 2012)

$$
\begin{aligned}
& \lambda_{1} L_{1}=\rho+\lambda_{1} N_{1}-I_{1}+\varepsilon_{L 1} \\
& \lambda_{2} L_{2}=\rho+\lambda_{2} N_{2}-\frac{\lambda_{2}^{2}}{\lambda_{1}^{2}} I_{1}+\varepsilon_{L 2}
\end{aligned}
$$

where $\lambda_{1}$ and $\lambda_{2}$ are the wavelength of the corresponding GPS signal; $L_{1}$ and $L_{2}$ are the received carrier phase observables in units of cycles; $N_{1}$ and $N_{2}$ are the integer phase ambiguity in units of cycles; $\rho$ is the geometric distance from the GPS receiver's antenna phase center at the epoch of signal reception to the GPS satellite's antenna phase center at the epoch of signal transmission; and $I_{1}$ is the ionospheric delay in units of length, while $\varepsilon_{L 1}$ and $\varepsilon_{L 2}$ combine the other terms in the previous equations, assumed to be random.

A cycle-slip can be obtained by differencing the carrier phase observation equations between two adjacent epochs, described as

$$
\begin{gathered}
\lambda_{1} \Delta L_{1}=\Delta \rho+\lambda_{1} \Delta N_{1}-\Delta I_{1}+\varepsilon_{L 1} \\
\lambda_{2} \Delta L_{2}=\Delta \rho+\lambda_{2} \Delta N_{2}-\frac{\lambda_{2}^{2}}{\lambda_{1}^{2}} \Delta I_{1}+\varepsilon_{L 2}
\end{gathered}
$$

where the operator $\Delta$ indicates the differencing between values associated with the current and the last epochs. The known terms in the measurements domain are the carrier phase measurements $L_{1}$ and $L_{2}$. Thus, the cycle-slip detection is based on the relation between the cycle-slip term $\Delta N_{1}$ and $\Delta N_{2}$ and the measurement term $\Delta L_{1}$ and $\Delta L_{2}$. The elimination of the geometric term $\rho$ is a key step for cycle-slip detection. The geometry term in (3.15 and 3.16) can also be estimated using other measurements unaffected by cycle-slips. Concerning the GPS observations, this term can be estimated by pseudorange data

$$
\begin{gathered}
P_{1}=\rho+I_{1}+\varepsilon_{P 1} \\
P_{2}=\rho+\frac{\lambda_{2}^{2}}{\lambda_{1}^{2}} I_{1}+\varepsilon_{P 2}
\end{gathered}
$$


The random terms in equation (3.19 and 3.20) are analogous to $\varepsilon_{L 1}$ and $\varepsilon_{L 2}$. Differencing the pseudorange observation equations between two consecutive epochs, one finds

$$
\begin{gathered}
\Delta P_{1}=\Delta \rho+\Delta I_{1}+\varepsilon_{P 1} \\
\Delta P_{2}=\Delta \rho+\frac{\lambda_{2}^{2}}{\lambda_{1}^{2}} \Delta I_{1}+\varepsilon_{P 2}
\end{gathered}
$$

In comparison with (3.17 and 3.18), the differences in the code observation equations lie in the opposite sign of ionospheric delay, the much larger thermal noise and multipath errors. Differencing equations (3.17) and (3.21), (3.18) and (3.22), assuming that the ionospheric delay does not substantially change between consecutive epochs (so that their differences can be incorporated into the random components), and rearranging the terms, one gets

$$
\begin{aligned}
& \Delta N_{1}=\frac{\lambda_{1} \Delta L_{1}-\Delta P_{1}}{\lambda_{1}}+\varepsilon_{1} \\
& \Delta N_{2}=\frac{\lambda_{2} \Delta L_{2}-\Delta P_{2}}{\lambda_{2}}+\varepsilon_{2}
\end{aligned}
$$

In expressions (3.23) and (3.24), the terms $\varepsilon_{i}$ (where $i=L_{1}, L_{2}, P_{1}, P_{2}$ and their differences) can be determined from definitions (3.15) to (3.22). Thus, if the first terms of the right sides of equations (3.23) and (3.24) substantially exceed the seconds, there are occurrences of cycle slips, which must be corrected (Blewitt, 1990).

Dual-frequency GPS receivers present a twofold superiority over singlefrequency receivers for cycle-slip detection. Firstly, the geometry term $\rho$ and the non-dispersive errors can be fully eliminated by a geometry-free phase combination. Secondly, the carrier phase measurements present much lower noise error than the code data (Dai, 2012). The geometry-free combination provides

$$
\lambda_{1} \Delta L_{1}-\lambda_{2} \Delta L_{2}=\lambda_{1} \Delta N_{1}-\lambda_{2} \Delta N_{2}-\left(1-\frac{\lambda_{2}^{2}}{\lambda_{1}^{2}}\right) \Delta I_{1}
$$

The cycle-slips occurring either on $L_{1}, L_{2}$, or both signals can be detected if the following condition holds 


$$
\left|\lambda_{1} \Delta L_{1}-\lambda_{2} \Delta L_{2}\right|>a \sigma_{c o m b}
$$

where $a$ is a multiplication factor determining the confidence level, set to 4 in the program source code,

$$
\sigma_{c o m b}=\sqrt{2} \sqrt{\lambda_{1}^{2}-\lambda_{2}^{2}} \sigma_{L 1}
$$

In the last expression, $\sqrt{2}$ reflects the between-epoch differencing and $\sigma_{L 1}$ stands for the standard deviation of carrier phase thermal noise.

Dual-frequency signals also allow a cycle slip determination based on the fact that the removal of the most likely value of cycle slip from the carrier phase data could yield the minimal residuals of geometry free combination. The formulation of search space of cycle slip candidates is expressed in detail in (Teunissen, 1995). Removing the cycle slip candidates from the carrier phase data should make the repaired carrier phase data most possibly pass the cycle slip detection tests (Dai, 2012).

\subsubsection{Leveling of the Carrier Phase with the Pseudorange}

The difference of the carrier phases $\left(L_{2}-L_{1}\right)$ is less noisy than the one provided by $\left(P_{2}-P_{1}\right)$, but does not provide the absolute slant TEC. Additionally, discontinuities (jumps) in the integer values of $L_{1,2}$, known as cycle slips, often arise. Cycle-slip corrections can typically be made with the aid of the pseudorange difference measurement, which is unambiguous but noisier (Ciraolo et al., 2005; Ma and Maruyama, 2003). To retain the accuracy for the slant $T E C_{s l}$, a baseline $B_{r s}$ is calculated as the average differences between pseudorange-derived $T E C_{s l p_{i}}$ and phase-derived $T E C_{s l l_{i}}$,

$$
B_{r s}=\frac{\sum_{i=1}^{N}\left\langle T E C_{s l p_{i}}-T E C_{s l l_{i}}\right\rangle_{a r c} \sin ^{2} \varepsilon_{i}}{\sum_{i=1}^{N} \sin ^{2} \varepsilon_{i}}
$$

with $i=1,2, \ldots, N$, where $N$ is the number of continuous measurements contained in the arc, and $\varepsilon$ is the elevation angle. The notation \langle\rangle$_{\text {arc }}$ in (3.28) indicates that the involved values should only taken over a phase-connected arc (between 
successive cycle slips) (Ciraolo et al., 2005; Ma and Maruyama, 2003). The computed baseline $B_{r s}$ is then added to $T E C_{\text {sll }}$ to provide $T E C_{s l}$ (Mannucci et al., 1998)

$$
T E C_{s l}=T E C_{s l l}+B_{r s}
$$

\subsubsection{Absolute sTEC}

Once the relative $T E C_{s l}$ has been estimated, subtraction of the satellite and receiver differential instrumental code biases yields the calibrated STEC, equal to the number of electrons encountered along the line of sight between the satellite and receiver per $m^{2}$, expressed as

$$
\begin{gathered}
s T E C=\left(T E C_{s l}-b_{s}^{P}-b_{r}^{P}\right) \\
B_{i}=b_{s}^{P}+b_{r}^{P}
\end{gathered}
$$

where $b_{s}^{P}$ and $b_{r}^{P}$ are the instrumental differential code biases (DCB) of the GPS satellite $s$ and receiver $r$, respectively (Kenpankho et al., 2011), respectively, computed according to the procedure described below.

\subsubsection{Differential Code Biases}

The differential code bias $\left(B_{i}\right)$ of each receiver - satellite pair is obtained by comparing the hourly averages of uncalibrated TEC values from all of the satellite and single receiver combinations, using the weighted least mean square fitting (LMSQ) method (Otsuka et al., 2002).

The biases are removed from the measured TEC to derive the absolute TEC. The set of parameters $\overline{V_{k}}$ and $B_{i}$ are estimated by minimizing the squared error $E^{2}$. It will be assumed that the hourly average of vertical TEC $\left(\overline{V_{k}}\right)$ is uniform within an area covered by a receiver, defined as

$$
E=\sum_{i}^{N_{S}} \sum_{k}^{N_{t}} W_{k}^{i}\left[\overline{\frac{\left(T E C_{s l}\right)_{k}}{S(\varepsilon)}}-\overline{V_{k}}-\overline{\frac{1}{S(\varepsilon)}} B_{i}\right]^{2}
$$


In the above equation, $k=1,2, \ldots, N_{t}$ and $i=1,2, \ldots, N_{s}$, where $N_{t}$ is the number of hourly TEC averages; and $N_{s}$ is the number of satellites which are observed by the receiver. All variables with overlines denote average values. $W_{k}^{i}$ is the weighting function, can be expressed as

$$
W_{k}^{i}=\overline{\frac{1}{S(\varepsilon)}}
$$

This weighting function depends on the slant factor

$$
S(\varepsilon)=\frac{1}{\cos \left[\arcsin \left(\frac{R_{E} \cos \varepsilon}{R_{E}+H}\right)\right]}
$$

where $\varepsilon$ is the elevation angle, $R_{E}$ is the Earth radius, and $H$ is the height of the ionospheric layer, which is assumed to be $400 \mathrm{~km}$. It is assumed that the ionosphere is compressed into a thin shell over the peak of the ionospheric $\mathrm{F}$ layer.

Once $B_{i}$ is estimated using the weighted LMSQ method, it is also possible to estimate the differential code bias of the receiver using equation (3.31), in this work, the satellite instrumental code bias is taken from code bias files $\left(P_{1} C_{1}\right.$ and $\left.P_{1} P_{2}\right)$ estimated by the Center for Orbit Determination in Europe (CODE).

After the application of the LMSQ, if the minimum $s T E C_{\text {dailymin }}^{r}$ (relative TEC with the GPS satellite DCB calibrated but still biased with the receiver DCB) continues to be negative, it is possible to assume that the receiver DCB is equal to that value (Rideout and Coster, 2006; Ciraolo et al., 2007). Generally, the DCB calculated from the ZERO TEC method can be expressed as follows

$$
B_{i}=0-s T E C_{\text {dailymin }}^{r}
$$

where $s T E C_{\text {dailymin }}^{r}$ is the daily minimum of the relative TEC. This simple method will be combined with the LMSQ fitting method. However, the $S T E C_{\text {dailymin }}^{r}$ might sometimes be an outlier. Thus, the derived $B_{i}$ is not always 
reliable. Outliers are mainly caused by the leveling errors, which are associated with cycle slip, multipath effect, and observational noise. For this reason, the DCBs of each receiver are estimated using the measurements corresponding to a $35^{\circ}$ satellite elevation mask and a local-time window between $6 \mathrm{~h}$ and $18 \mathrm{~h}$, avoiding ionospheric scintillation periods and negative TECUs.

After the application of the above procedures, the biases are removed from measured TEC to derive the absolute sTEC (Otsuka et al, 2002).

\subsubsection{Absolute vTEC}

The slant TEC (sTEC) depends on the ray path geometry through the ionosphere. To estimate a version of this parameter that does not depend on the elevation angle of the ray path, the equivalent vertical (vTEC) is determined (Ma et al., 2002). Each slant TEC value will be transformed into a vTEC one, by using the simple mapping function depicted in Figure 3.4. This model is related with a latitude and longitude of the ionospheric pierce point (IPP), assuming that the ionosphere is compressed into a thin shell over the F peak layer of the ionosphere.

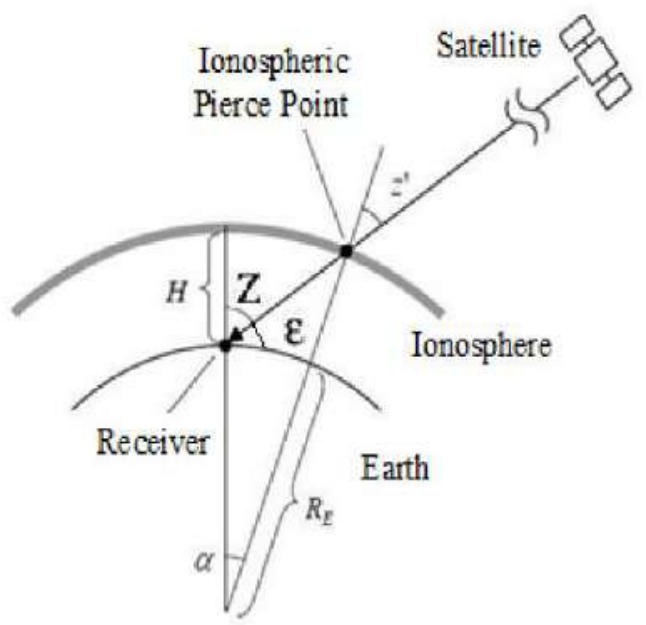

Figure 3.4: Geometry of a GPS satellite, the ionosphere and a receiver. Here, the Ionospheric Pierce Point (IPP) is the intersection between the ray path and the ionospheric shell, $\mathrm{Z}$ is zenithal angle and $\varepsilon$ is the elevation.

[Reproduced from http://gpspp.sakura.ne.jp/tutorial/html/gps_symp_2005_2]

To obtain vTEC, one first computes the sTEC from equation (3.30). The instrumental code biases are eliminated from the data to calibrate the experimental slant TEC obtained from GPS observations, and the calibrated vTEC (without instrumental code bias) is then finally determined by (Ma et al., 2002) 


$$
v T E C=\frac{s T E C}{S(\varepsilon)}
$$

where vTEC is measured in TEC units $\left(1\right.$ TECU $=10^{16}\left(\right.$ electrons $\left./ \mathrm{m}^{2}\right)$.

\subsection{Ionospheric Pierce Point}

The ionospheric pierce point (IPP) is the intersection of the receiver-tosatellite ray path with the ionosphere, assumed a thin layer, located at the mean altitude $\mathrm{H}(400 \mathrm{~km})$. It is possible to determine the position of the IPPs as a function of the station and satellite geographic coordinates. Moreover, it is necessary to know the satellite azimuth and elevation angle. The geographic latitude and longitude of the IPP can be obtained by the following expressions (El-Gizawy, 2003; Prol et al., 2017)

$$
\begin{gathered}
\Phi_{I P P}=\sin ^{-1}\left[\sin \Phi_{\mathrm{r}} \cos \Psi+\cos \Phi_{\mathrm{r}} \sin \Psi \cos \mathrm{Az}\right] \\
\lambda_{I P P}=\lambda_{r}+\sin ^{-1}\left(\frac{\sin \Psi \sin A z}{\cos \Phi_{I P P}}\right) \\
\Psi=\frac{\pi}{2}-\varepsilon-\sin ^{-1}\left(\frac{\mathrm{R}_{\mathrm{E}}}{\mathrm{R}_{\mathrm{E}}+\mathrm{H}} \cos (\varepsilon)\right)
\end{gathered}
$$

where $\Phi_{I P P}$ and $\lambda_{I P P}$ represent the IPP latitude and longitude, respectively, $\Phi_{\mathrm{r}}$ and $\lambda_{r}$ are the latitude and longitude of GNSS receiver, $A z$ and $\varepsilon$ are the satellite azimuth and elevation angle, $\mathrm{R}_{\mathrm{E}}$ is mean Earth radius, $\mathrm{H}$ is the mean altitude of the ionospheric layer.

\subsection{Data}

Data from the Rede Brasileira de Monitoramento Contínuo of the Instituto Brasileiro de Geografia e Estatística (RBMC/IBGE) were obtained to study the variation of the vertical TEC as a function of position, local time, season and solar activity over the Brazilian region. This data was obtained through Dr. Patrícia M. 
S. Negreti, under the supervision of Prof. Eurico R. de Paula (Division of Aeronomy, National Institute of Space Research).

The original data were stored in the Receiver Independent Exchange format (RINEX) format with 15-second sampling rate. The measurements were extracted from data collected using an elevation cut-off angle of $20^{\circ}$, to avoid multipath. In this chapter, RBMC RINEX files were used from January to December corresponding to the years 2002, 2008 and 2013. The deployed stations over the Brazilian territory used to estimate the vTEC are shown by red dots in Figure 3.5.

The data referring to the years 2002, 2008 and 2013 correspond to the maximum (cycle 23), minimum (cycle 23) and maximum (cycle 24) of solar activity, respectively. All levels of geomagnetic activity are represented in each year. The number of stations being used is very dynamic. Some stations had technical problems and therefore did not provide data for some periods of the present study. It is relevant to mention the existence of 15,50 , and 86 receiver stations in 2002, 2008 and 2013, respectively.

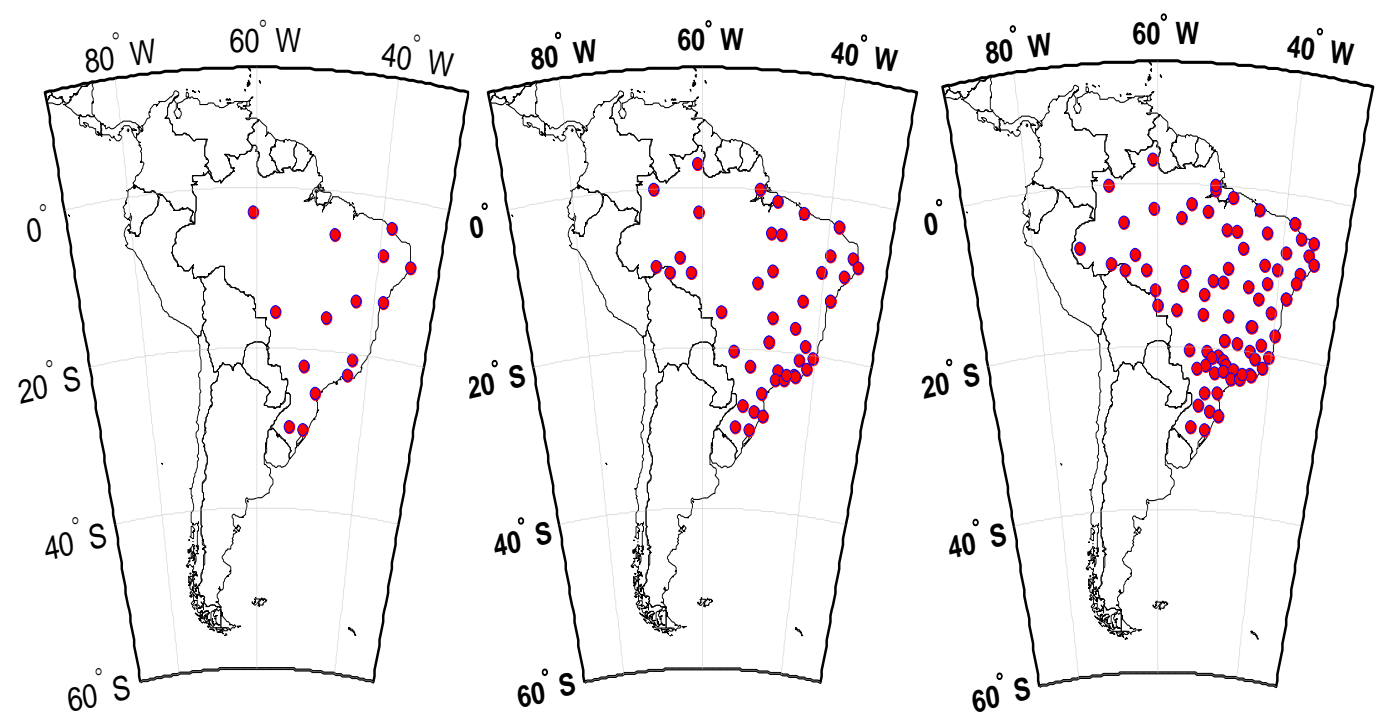

Figure 3.5: Positions of the ground-based RBMC stations in 2002, 2008 and 2013.

In this work, the vTEC will be clasified according to the geomagnetic DIP latitude. Figure 3.6 shows the map of geomagnetic DIP latitude. The green contour line connects points where the inclination is $0^{\circ}$ (geomagnetic equator). To the north of the equator, the red contour lines denote regions with positive 
inclination (magnetic dip angle is down). To the south, blue contour lines represent regions with negative inclination values (magnetic dip angle is up).

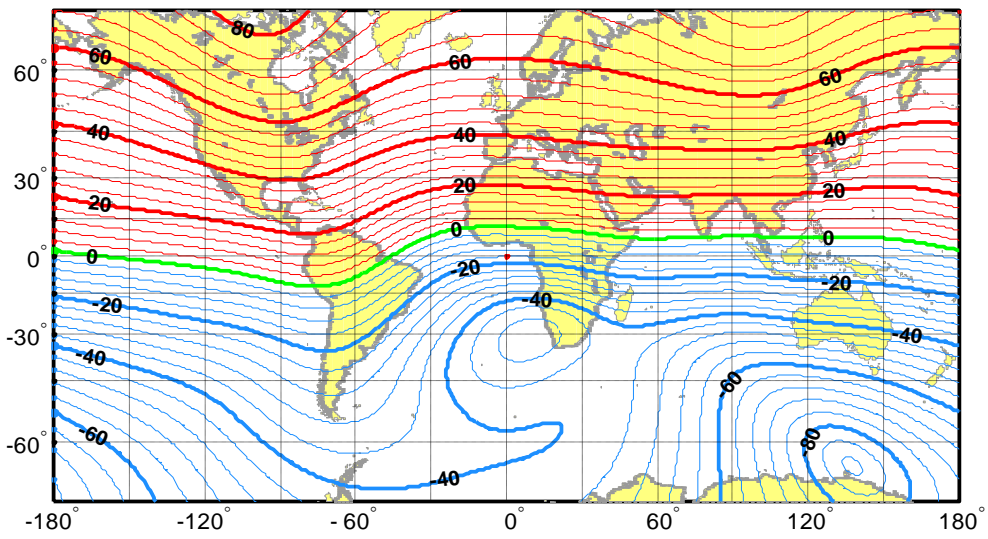

Figure 3.6: Map of geomagnetic DIP latitude.

\subsubsection{Geomagnetic and Solar Indices}

The magnetic field of the Earth responds constantly to the disturbances that propagate from the Sun. When the disturbance occurs, various components of the Earth's field change, releasing magnetic energy and thereby accelerating charged particles to high energies. These particles, being charged, are forced to stream along the geomagnetic field lines causing variation in the geomagnetic activity.

The Planetary K-index $\left(K_{p}\right)$, is an average index from a network of geomagnetic observatories, used to characterize the magnitude of geomagnetic disturbances in the range $0-9$, with 1 being calm and 5 or more indicating a geomagnetic storm.

The solar radio flux at $10.7 \mathrm{~cm}(2800 \mathrm{MHz})$ is a good indicator of solar activity. The F10.7 radio emissions track important emissions from the solar atmosphere that impact and modify the upper atmosphere and the ionosphere. The F10.7 index can vary from below $50 \mathrm{sfu}$, to above $300 \mathrm{sfu}$, where $1 \mathrm{sfu}$ (solar flux unit) corresponds to $10^{-22}\left[\mathrm{Wm}^{-2} \mathrm{~Hz}^{-1}\right]$.

The Space Weather Prediction Center/ National Oceanic and Atmospheric Administration (SWPC/NOAA) maintains tables of F10.7 and geomagnetic indices, including the Planetary K-index since 1994. Figure 3.7 shows the international solar radio flux $(10.7 \mathrm{~cm})$ and the planetary magnetic field $\mathrm{Kp}$ indices from January 01, 2000 to December 31, 2016. The year 2002 corresponds to the maximum of solar activity during cycle 23 (first pairs of vertical red lines). On the 
other hand, the year 2008 was associated with minimum solar activity, at the transition from cycles 23 to 24 (pair of vertical green lines). Finally, the year 2013 is close to the maximum of solar activity values during cycle 24 (second pair of vertical red lines).
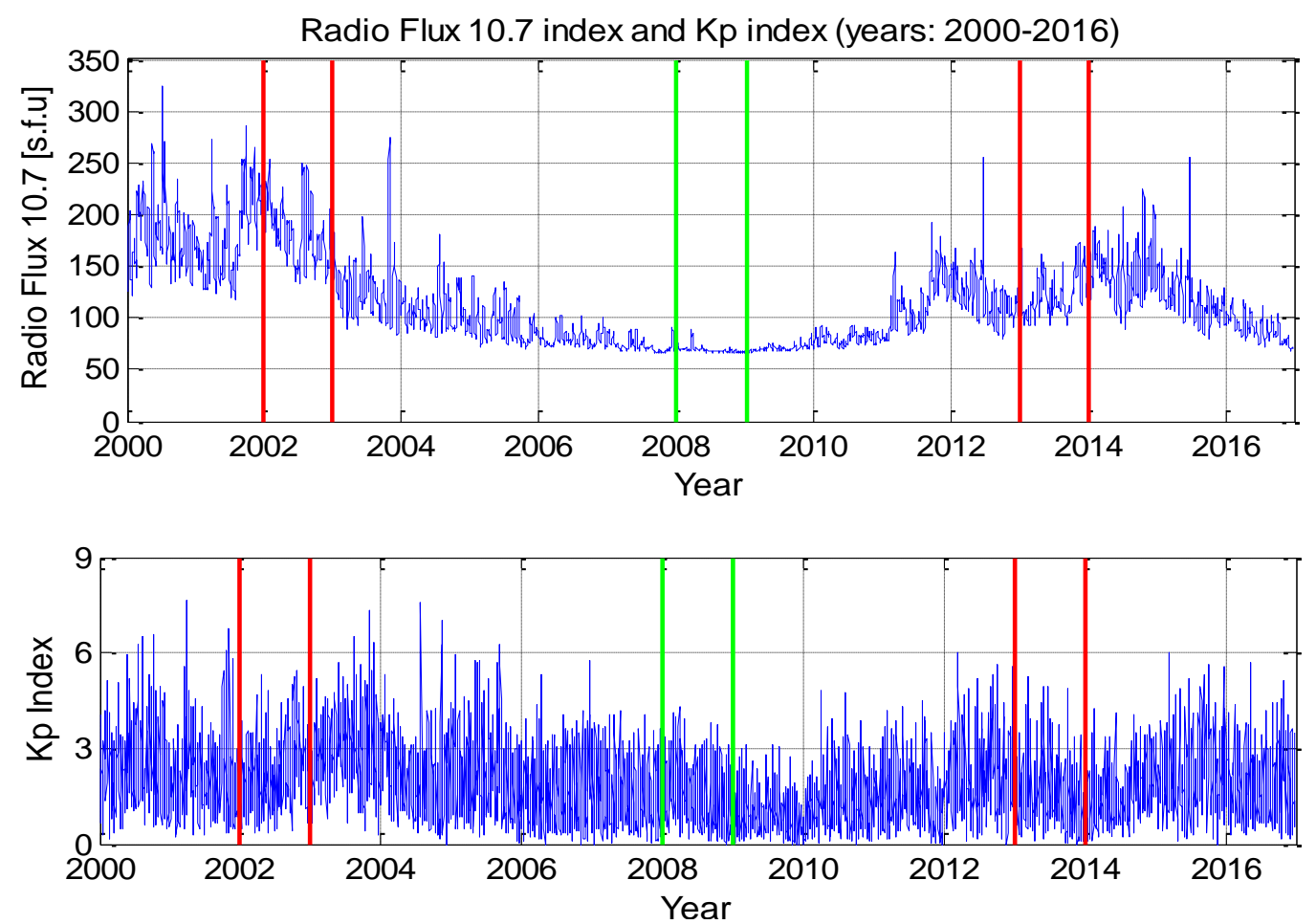

Figure 3.7: Solar and geomagnetic indices (F10.7 and Kp indices).

\subsubsection{GPS Satellite Positions}

In the procedure to estimate the IPP is necessary to estimate the azimuth and elevation angle based on the position of the receiver. The positions of the GPS satellites were determined from the GPS Precise Orbit Files presented in the Standard Product (SP3) format, obtained from the International GNSS Service (IGS). However, the positions of the satellites are reported in 15-minute intervals. To circumvent this problem, the positions of the satellites are estimated every 15 seconds through cubic interpolation. The errors in the calculation of the weighting function based on the elevation angle (equation 3.33) are minimal. 


\subsection{Results}

\subsubsection{Estimation of Vertical TEC}

To estimate the ionospheric effects on GBAS, the variability of estimated vertical Total Electron Content (vTEC) at associated $400 \mathrm{~km}$ ionospheric pierce point (IPP) is studied through a quantitative and statistical analysis of dualfrequency GPS data from the RBMC network.

The positions of the RBMC network stations and the GPS satellites positions at a particular epoch expressed in ECEF coordinates are extracted from the information of RINEX and SP3 files, respectively. To estimate the slant TEC, the GPS observation time series are obtained from the RBMC data and are corrected for cycle slips, as well as satellite and ground receiver biases (Ma et al., 2003). Next, they are mapped into vTEC, through its product by a slant factor that depends on the zenith angle of the corresponding ray path and the altitude of the associated IPP.

This section presents results and analysis of vertical TEC for two different days, corresponding to high (September 11, 2002) and low (September 10, 2008) geomagnetic and solar activities. The associated solar and geomagnetic indices are shown in Table 3.1.

Table 3.1: Solar and geomagnetic indices for selected days.

\begin{tabular}{|c|c|c|c|}
\hline \multicolumn{2}{|c|}{ High Activity } & \multicolumn{2}{c|}{$\begin{array}{c}\text { Low Activity } \\
\text { Date: 10-Sep-2008 }\end{array}$} \\
\hline Date: 11-Sep-2002 & K$_{\mathbf{p}}$ index & F10.7 index & K$_{\mathbf{p}}$ index \\
\hline $219.0[\mathrm{sfu}]$ & $2.3-2.3-4.0-5.0-$ & $68.1[\mathrm{sfu}]$ & $1.7-1.3-1.0-1.0-$ \\
& $3.0-4.7-3.7-4.7$ & & $0.7-1.3-1.7-1.0$ \\
\hline
\end{tabular}

The vTEC values were estimated using measurements transmitted from all observed GPS satellites and received by the monitoring stations located in different magnetic dip latitudes summarized in the Table 3.2. 
Table 3.2: Description of receiver stations.

\begin{tabular}{|c|c|c|c|c|}
\hline Station & Location & \multicolumn{2}{|c|}{ Geographic Coordinates } & Dip Latitude \\
\hline IMPZ & Imperatriz, Brazil & $05.48^{\circ} \mathrm{S}$ & $47.48^{\circ} \mathrm{W}$ & $3.60^{\circ} \mathrm{S}$ \\
\hline BRAZ & Brasília, Brazil & $15.93^{\circ} \mathrm{S}$ & $47.82^{\circ} \mathrm{W}$ & $12.10^{\circ} \mathrm{S}$ \\
\hline RIOD & Rio de Janeiro, Brazil & $22.82^{\circ} \mathrm{S}$ & $43.30^{\circ} \mathrm{W}$ & $20.83^{\circ} \mathrm{S}$ \\
\hline
\end{tabular}

The positions of all the ground-based RBMC stations available in 2002 are plotted in the left panel of Figure 3.8 (red and blue symbols), while those listed in Table 3.2 are indicated by blue triangles. In adittion, the path position of the 400$\mathrm{km}$ IPPs for the IMPZ station and each GPS satellite (different color lines) on September 11, 2002 are plotted in the right panel.
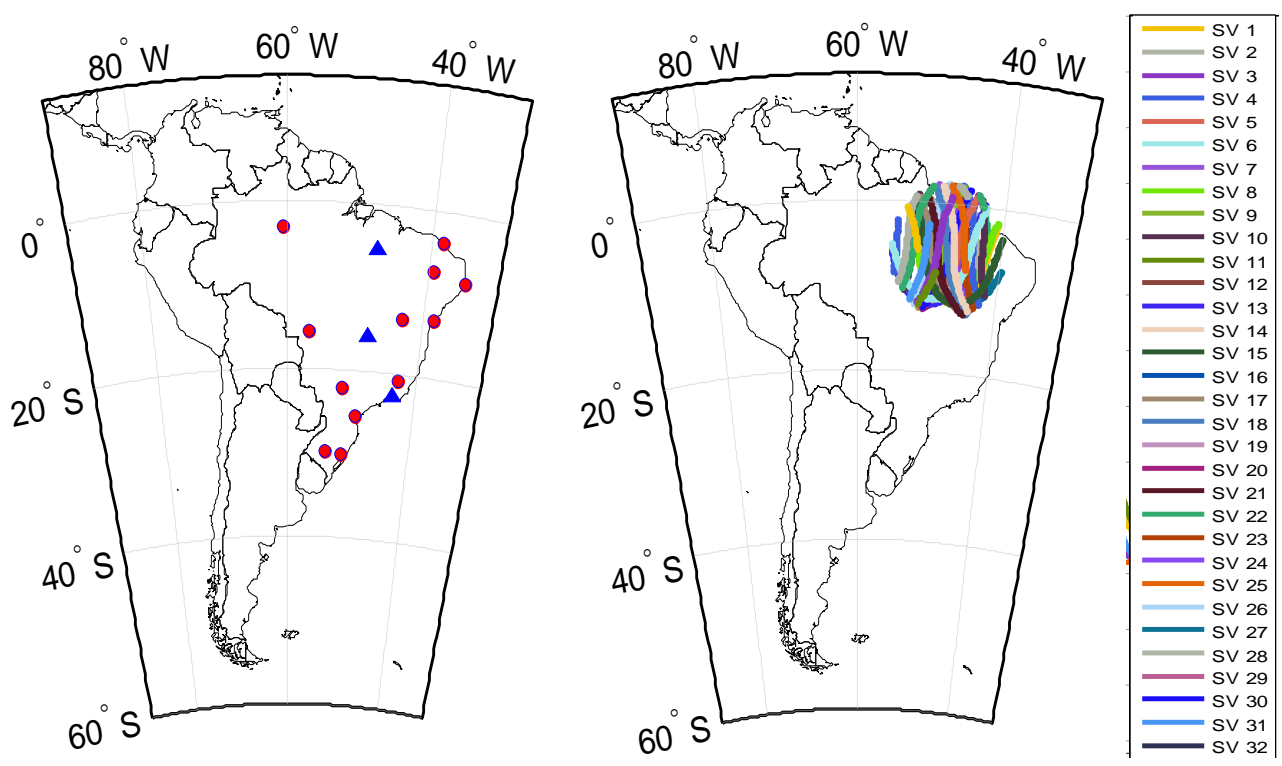

Figure 3.8: Left: positions of all the ground-based RBMC stations available in 2008 (red and blue symbols), while those listed in Table 3.2 are indicated by blue triangles; Right: path positions of the 400-km IPPs for the IMPZ station and each GPS satellite (different line color) on September 11, 2002. 


\subsubsection{Detection and Repair of Cycle Slips}

This section presents some results of the detection and correction of the cycle slips that occur due to signal blockage, scintillation or some other disturbing factor, using the data from BRAZ receiver station on September 11, 2002 (day with high geomagnetic and solar activities). In Figure 3.9, each subplot represents the combination of the carrier phase $\mathrm{L}_{1}$ and $\mathrm{L}_{2}$ (blue points) and the detected abnormal jumps that may occur in the sequence of combinations (red points) that are received from each satellite in orbit.
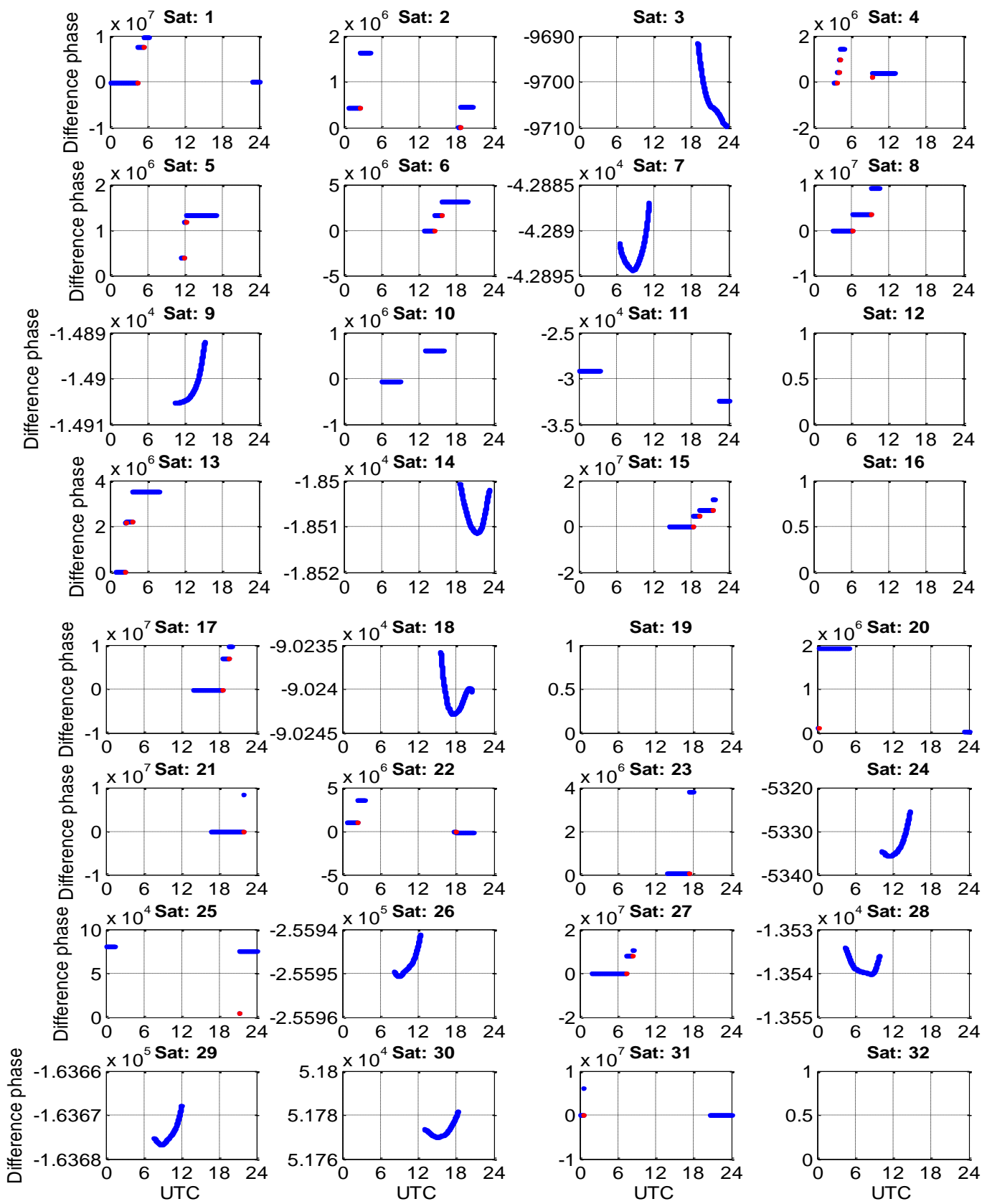

Figure 3.9: Detection of cycle slips for the BRAZ station on 11 September 2002. 
Many satellites present cycle slips during the day with high solar and geomagnetic activity, except SV3, SV7, SV9, SV10, SV11, SV14, SV18, SV24, SV26, SV28, SV 29 and SV 30 because these presents uninterrupted arcs. After the cycle slips detected, these were corrected. In Figure 3.10, each subplot represents the combination of the carrier phase $\mathrm{L}_{1}$ and $\mathrm{L}_{2}$ with the corrections of cycle slips (red points) that are received from each satellite in orbit.
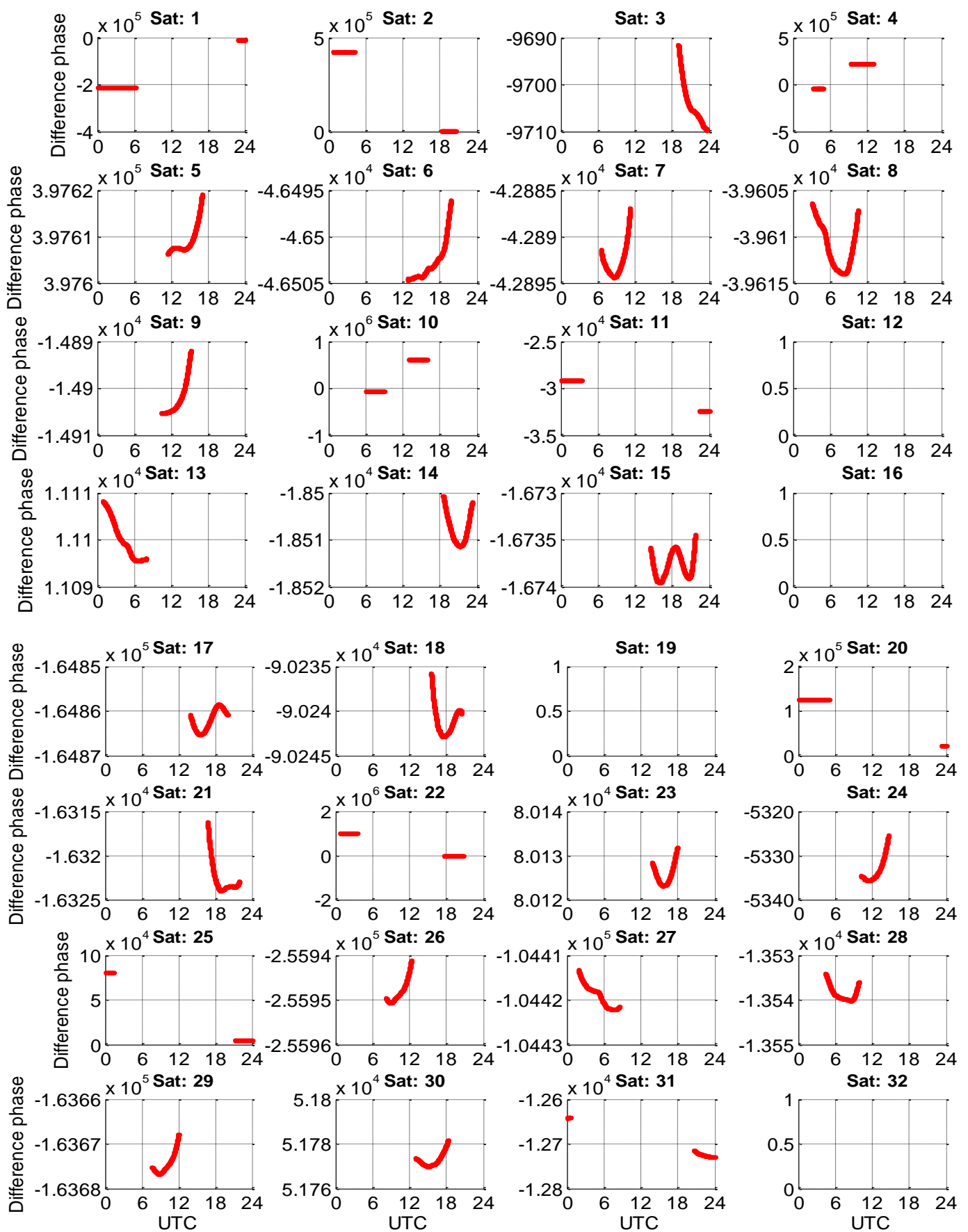

Figure 3.10: Cycle slip repaired for the BRAZ station on 11 September 2002. 
Figure 3.10 shows results of the correction of the cycle slips, where the abnormal jumps are corrected. For example, the subplot of satellite SV15 in Figure 3.9 displays 3 jumps in the combination of carrier phases $\mathrm{L}_{1}$ and $\mathrm{L}_{2}$ at and the corresponding subplot of Figure 3.10 displays the correction for these 3 jumps at 18:06:30 UTC, 19:17:30 UTC and 21:24:45 UTC, after this correction, SV15 presents an uninterrupted arc.

\subsubsection{Results for Differential Code Biases of the Receivers}

The code bias is a delay that affects the estimation of TEC in the ionosphere, as described in more detail in Section 3.3.6. Figure 3.11 illustrates the results of differential code bias estimations through the LMSQ and Zero method (red dots) for BRAZ receiver station (Geographic coordinates: $15.93^{\circ} \mathrm{S}, 47.82^{\circ} \mathrm{W}$; dip latitude $12.10^{\circ} \mathrm{S}$ ), using data for each day of the year 2002, 2008 and 2013.

The International GNSS Service (IGS) works in the production of IONEX files that, in addition to the estimates of the vTEC values, also includes the DCBs of the satellites and receivers of the network. The DCBs of the IONEX archives of the IGS (Center for Orbit Determination in Europe (CODE) - European Space Agency) are presented with a daily resolution. To validate the methodology described in the previous paragraph, the DCB values for the same receiver station are obtained from the IONEX file and represented by blue dots in the three panels of Figure 3.11.

The mean and standard deviation values of the DCBs estimated in nanoseconds (ns) for the BRAZ station by the combined methods and CODE for the years 2002, 2008 and 2013 are compared in the Table 3.3.

Table 3.3: Statistics of estimated DCB.

\begin{tabular}{|c|c|c|c|c|}
\hline Year & $\begin{array}{c}\text { Mean (combined } \\
\text { method) (ns) }\end{array}$ & $\begin{array}{c}\text { Standard Deviation } \\
\text { (combined method) } \\
(\mathbf{n s})\end{array}$ & $\begin{array}{c}\text { Mean } \\
\text { (CODE) }(\mathbf{n s})\end{array}$ & $\begin{array}{c}\text { Standard } \\
\text { Deviation } \\
\text { (CODE) }(\mathbf{n s})\end{array}$ \\
\hline 2002 & -13.24 & 2.76 & -12.31 & 2.96 \\
\hline 2008 & 17.68 & 0.87 & 18.23 & 1.76 \\
\hline 2013 & $17.32 /-14.80$ & $1.42 / 1.63$ & $17.20 /-14.12$ & $1.45 / 1.12$ \\
\hline
\end{tabular}


The results shown in Figure 3.11 and Table 3.3 indicate a good agreement between DCBs estimated by the described algorithm and the one available from CODE. Also, the standard deviation estimated using the LMSQ is reduced for the three cases. The standard deviation estimated for BRAZ station (2.76 ns) in 2002 shows a large dispersion of data as depicts the first panel in Figure 3.11. Additionally, the third panel of Figure 3.11 shows a jump, it is due to a change of the receiver.
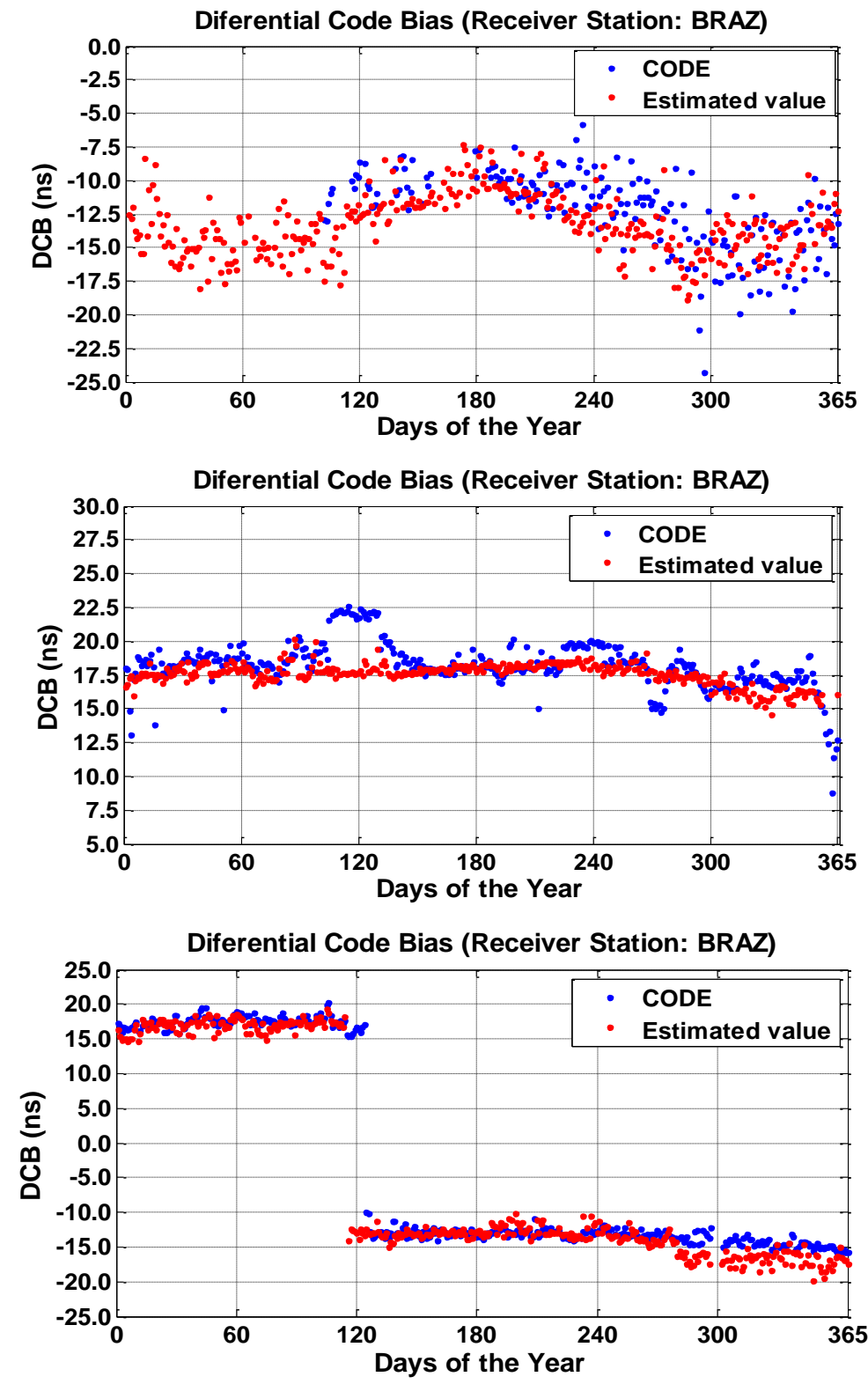

Figure 3.11: DCB estimated through the combined methods (red dots) and CODE (blue dots) method for the BRAZ station in 2002 (top), 2008 (middle) and 2013 (bottom). 
Also, the variation of the DCBs according to the geomagnetic latitude of the receiver was studied, results from São Luis (SALU), Fortaleza (BRFT), Brasília (BRAZ), Recife (RECF) and Cachoeira Paulista (CHPI) are presented by Surco et al. (2019). The results indicate that CHPI and BRAZ stations present large standard deviations than the other stations. For the former station, these variations are due may be due to its location under the crest of the equatorial ionization anomaly (EIA), generally within the geomagnetic latitude interval $\left(-12^{\circ},-20^{\circ}\right)$.

The objective of the study reported in this section is to compare the GPS vertical TEC estimated from different station data on the two selected days: 1) 11 September 2002 (left); 2) 11 September 2008 (right). It will be shown that the ionospheric effects over low and equatorial latitudes may be reasonable different from the ones at mid-latitudes, due the presence of the equatorial ionization anomaly (EIA). To analyze these effects, the GPS measurements by the three stations IMPZ, BRAZ and RIOD are studied.

Figure 3.12 represent the calibrated vTEC for the three receiver stations listed in Table 3.2, all available GPS satellites, and the two selected days. The variation of vTEC for each GPS satellite is represented by a different color line throughout each day. It is observed that the maximum vTEC values for the IMPZ, BRAZ and RIOD stations are 99.40 TECUs, 111.05 TECUs and 129.97 TECUs, respectively. As expected, these values are relatively small for the station closest to the geomagnetic equator and large for that near the southern crest of the EIA during a day with high solar and geomagnetic activity.

On the other hand, IMPZ, BRAZ and RIOD receiver stations presents low values of TECUs during a day with low solar and geomagnetic activity, these achieves 24.80 TECUs, 20.96 TECUs and 17.20 TECUs, respectively. For this case, the values estimated during a day with low activity represents $24.9 \%, 18.9 \%$ and $13.3 \%$ from a day with high activity, respectively. 

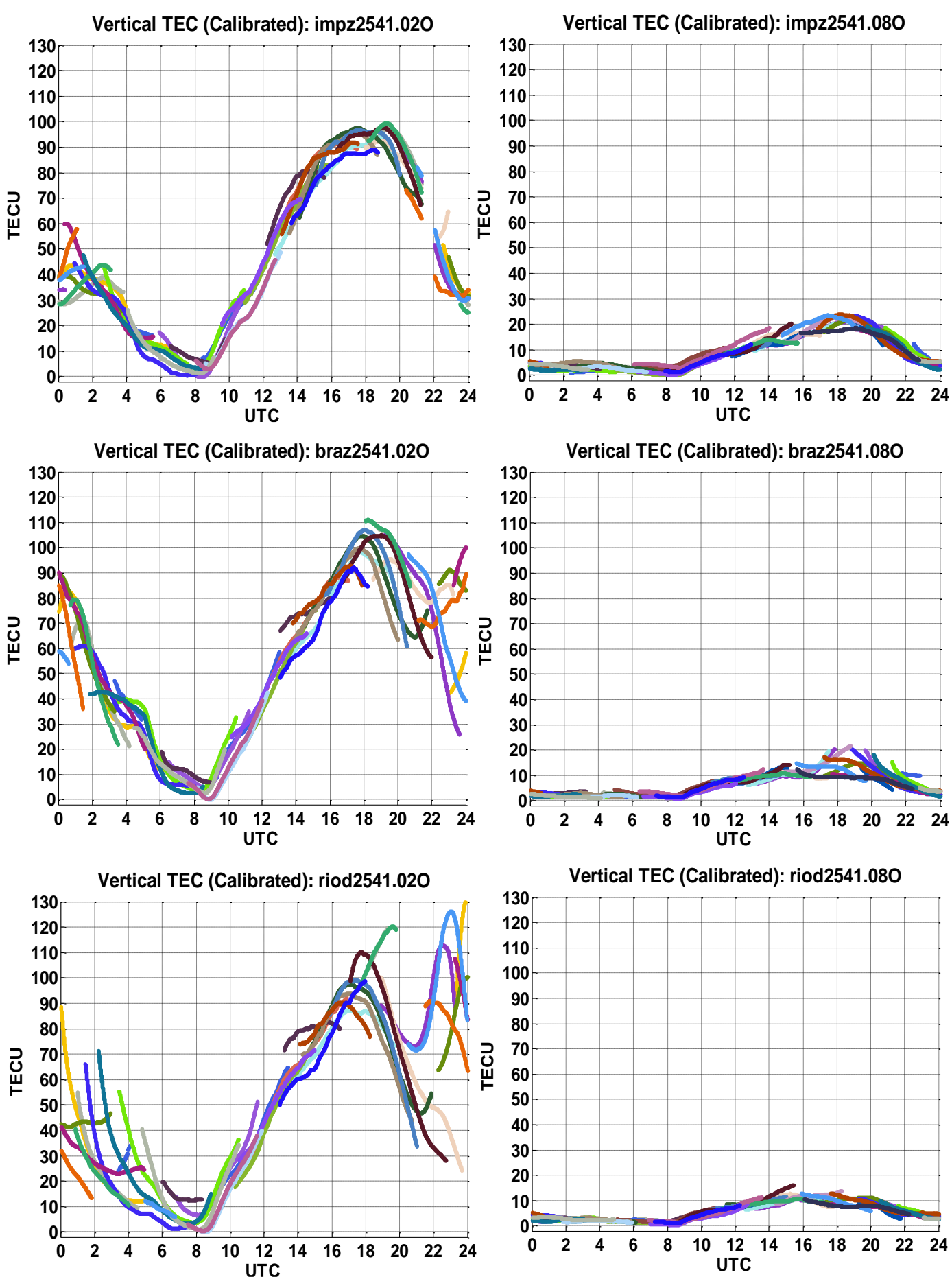

Figura 3.12: GPS vTEC (one-line color for each GPS satellite) for IMPZ (top), BRAZ (middle) and RIOD (bottom) stations on September 11, 2002 (high solar activity, left) and September 10, 2008 (low solar activity, right), respectively.

Figure 3.13 shows the skyplot of the available satellites GPS available (different color lines) tracked by RIOD station on September 11, 2002. 


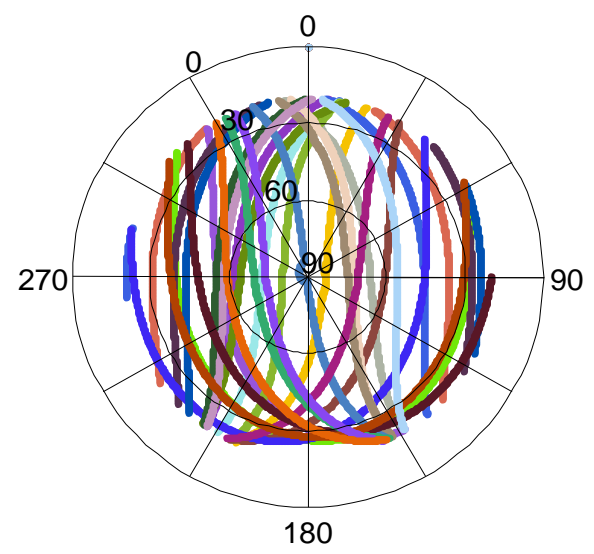

Figure 3.13: Skyplot of the GPS satellite orbits on September 11, 2002.

\subsubsection{Maximum Daily Vertical TEC}

The vertical TEC is an important indicator of ionospheric effects on the propagation of GPS signals in the space. For example, the TEC induces delays to the signal. That is, the maximum vTEC can be used as an indication of the maximum delay that GPS signals may experiment due to ionospheric effects. Additionally, it also displays the ionospheric seasonal dependence. Figure 3.14 shows the maximum daily value of vTEC for the IMPZ (red), BRAZ (blue) and RIOD (green) stations for the years 2002 (top), 2008 (middle) and 2013 (bottom).

Figure 3.14 (top) shows that the maximum values of vTEC for 2002 (year with high solar of cycle 23) vary between approximately 38 TECUs and 178 TECUs for the RIOD station; and 50 TECUs and 130 TECUs for the IMPZ and BRAZ stations. By contrast, Figure 3.14 (middle) shows that the maximum values of vTEC for 2008 (year with low solar activity of cycle 23) vary between approximately 10 TECUs and 55 TECUs for the three stations. In addition, the maximum values of vTEC for 2013 (year with high solar activity of cycle 24) vary between approximately 25 TECUs and 135 TECUs for the RIOD station; and 40 TECUs and 105 TECUs for the IMPZ and BRAZ stations as shows Figure 3.14 (bottom).

According to the values of TECUs estimated for the year 2002 (close to the maximum of solar activity during cycle 23) and for the year 2013 (close to the maximum of solar activity values during cycle 24 ), it is evident that the cycle 23 presented more geomagnetic and solar activity than the cycle 24 . 

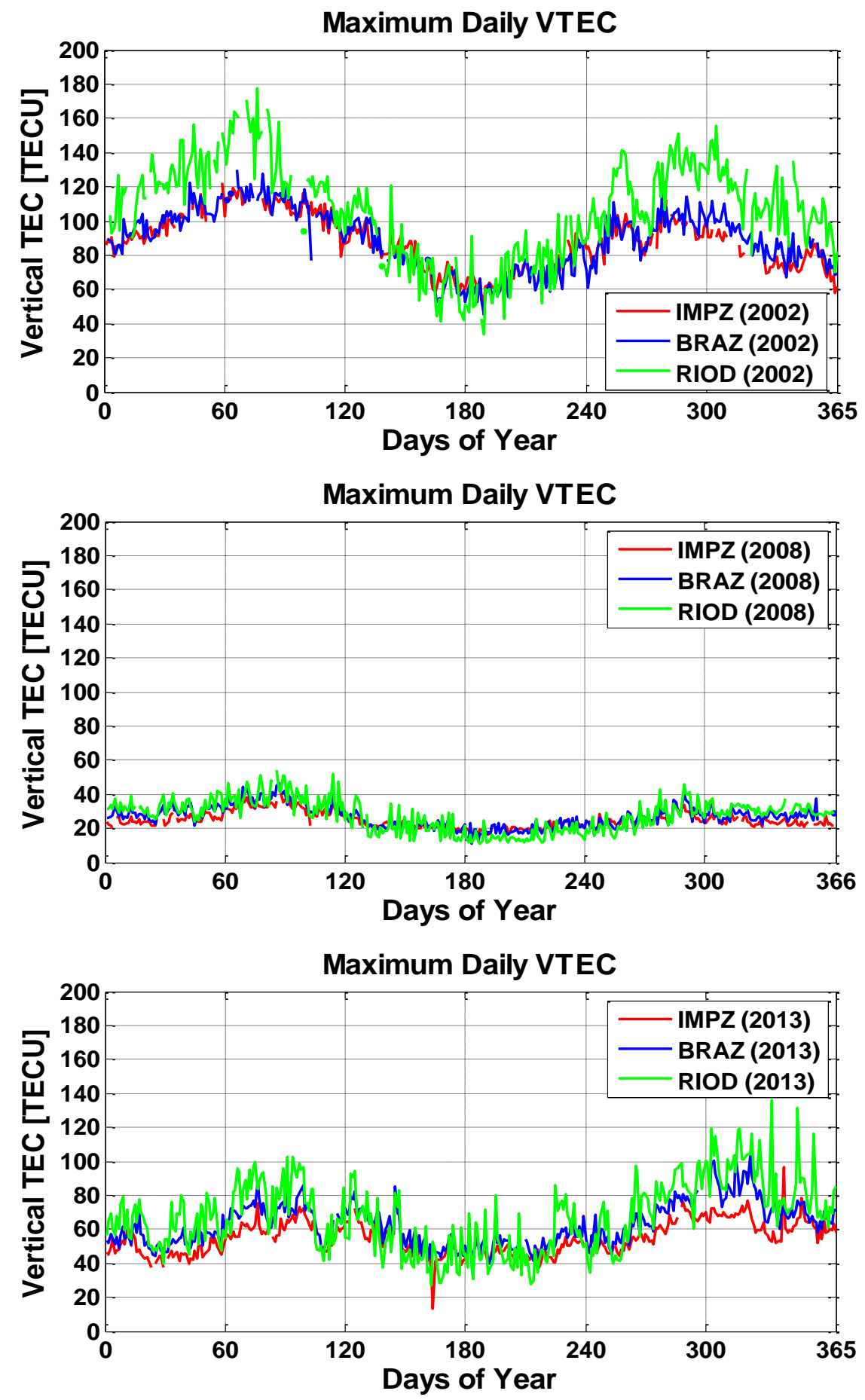

Figure 3.14: Maximum daily values of vTEC for the IMPZ (red), BRAZ (blue), and RIOD (green) stations in 2002 (top), 2008 (middle) and 2013 (bottom). 


\subsubsection{Comparison Between vTEC Measurements and Corresponding Predictions by the International Reference lonosphere Model for the Brazilian Region}

This section examines the prediction capability of the latest version of International Reference Ionosphere (IRI 2016) model (Bilitza, 2016) for vertical TEC variation over the Brazilian Region during high solar activity years of cycles 23 and 24 (2002 and 2013, respectively) and a low solar activity year (2008). This study has been carried out by comparing vTEC values predicted by IRI and estimated from data of the dual-frequency GPS receivers of the RBMC stations installed over Brazilian region in different magnetic latitudes, as shown in Figure 3.8 .

The IRI model is an international empirical model used to estimate averages of ionospheric parameters (such as TEC) for several solar and magnetic activity conditions, different locations and time variations. To enhance the capacity of the model, improvements have been developed through the processing of all worldwide available data. As a result, a new version of the model (IRI 2016) has been released by incorporating some improvements that increase its capacity and performance.

The vTEC predictions provided by the IRI model are calculated using the location, date, and time period as inputs. The vTEC of the IRI-2016 model is based on the current plasma frequency measurements F2 (foF2), the NeQuick option for topside electron density using Intercosmos 19 topside sounder data and the International Radio Consultative Committee (CCIR) data from the worldwide network of ionosonde are used for the $\mathrm{F}_{2}$ peak density. The IRI model is driven by several solar and ionospheric indices: the sunspot number $\mathrm{R}$, the ionosonde-based ionospheric global (IG) index (index based on foF2 measurements from a dozen ionosondes correlated with the CCIR foF2 map), the solar radio flux at $10.7 \mathrm{~cm}$ wavelength F10.7 (measurements at $2800 \mathrm{MHz}$ made by Dominion Radio Astrophysical Observatory) (Bilitza, 2016).

The differences between measured and modeled vTEC values are determinated every 15 seconds. Figure 3.15 shows the vTEC estimated from the IMPZ, BRAZ, and RIOD data, for all visible satellites (lines with different colors) and estimated by the IRI model for the same station and satellites (red lines). The 
left and right panels compare results from 11 September 2002 (day with high solar and magnetic activity) and 10 September 2008 (day with low solar and magnetic activity). The values of the solar and magnetic indices for both cases have been previously listed in Table 3.1.
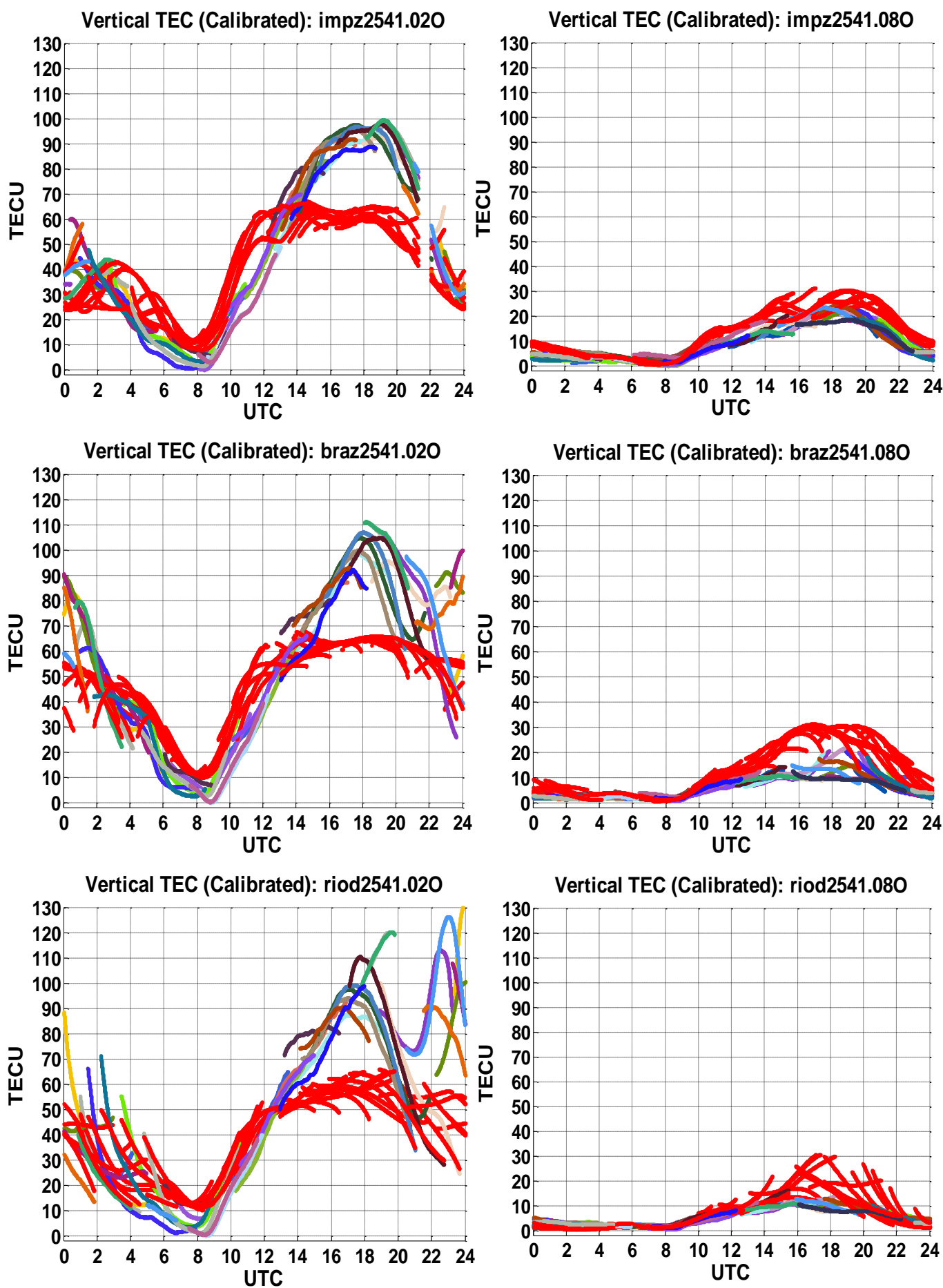

Figura 3.15: RBMC (lines with different colors) and IRI (red lines) vTEC for the IMPZ (top), BRAZ (middle), and RIOD (bottom) stations on 11 September 2002 (left) and 10 September 2008 (right), respectively. 
Figure 3.15 shows that the vTEC estimated using measurements can exceed approximately 70 TECUs the values estimated by the IRI model, in the intervals 00:00 UTC to 04:00 UTC and 14:00 UTC to 24:00 UTC in the case of the station of Rio de Janeiro (RIOD) in a year of high solar activity. On the other hand, the vTEC estimated using measurements for Brasilia station (BRAZ) present an underestimation, approximately 16 TECUs in relation to the values calculated by the IRI model during a day with low solar activity.

Next, this work will also present statistical distributions of residuals of measured values of vTEC in relation to those provided by IRI 2016. This residual is estimated as $\left(\mathrm{vTEC}_{\mathrm{RBMC}}-\mathrm{vTEC}_{\mathrm{IRI}}\right)$. This analysis considers different combinations of ranges of the following geophysical parameters: (i) geomagnetic latitude of IPP; (ii) local time; (iii) EPB season in the Brazilian sector, closely associated with that of ionospheric scintillation; (iv) solar activity, represented by the F10.7 index; (v) geomagnetic activity, represented by the Kp index. Data from all the RBMC stations operating during the intervals: (1) 01 January 2002 to 31 December 2002; (2) 01 January 2008 to 31 December 2008; and (3) 01 January 2013 to 31 December 2013; were used. Statistical distributions will be parameterized into 72 classes resulting from all combinations of the following ranges of geophysical parameters:

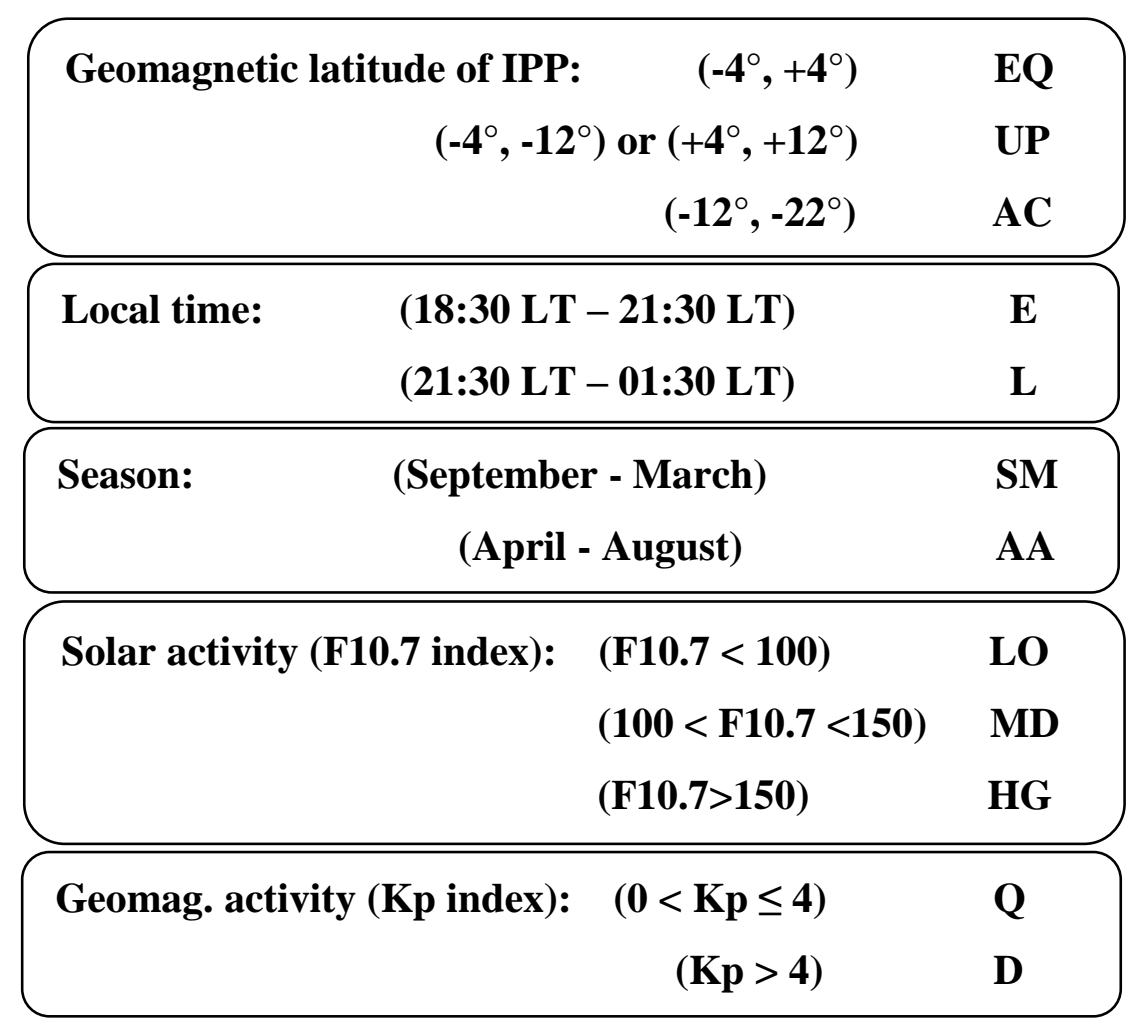


The 72 statistical combinations of all geophysical parameters denoted by the following symbology: equatorial (EQ), anomaly crest (AC), up (UP) for geomagnetic latitude; early (E) and late (L) for local time; September to March (SM) and April to August (AA) for EPB season; low (LO), middle (MD) and high (HG) for solar activity; and quite (Q) and (D) for geomagnetic activity are shown in Table 3.4.

Table 3.4: Combinations of geophysical parameters.

\begin{tabular}{|c|c|c|c|c|c|c|c|c|c|c|c|c|c|c|c|c|c|}
\hline & $\mathrm{Gm}$ & & & & & & $\mathrm{Gm}$ & & & & & & $\mathrm{Gm}$ & & & & \\
\hline No & Lt & LT & Seas & F10.7 & Kp & No & $\Delta \tau$ & LT & Seas & F10.7 & Kp & No & Lt & LT & Seas & F10.7 & Kp \\
\hline 1 & EQ & $\mathbf{E}$ & SM & LO & $\mathbf{Q}$ & 25 & UP & E & SM & LO & $\mathbf{Q}$ & 49 & $A C$ & E & SM & LO & $\mathbf{Q}$ \\
\hline 2 & EQ & $\mathbf{E}$ & SM & LO & D & 26 & UP & E & SM & LO & D & 50 & $\mathrm{AC}$ & E & SM & LO & D \\
\hline 3 & EQ & $\mathbf{E}$ & SM & MD & $\mathbf{Q}$ & 27 & UP & E & SM & MD & $\mathbf{Q}$ & 51 & $\mathrm{AC}$ & E & SM & MD & $\mathbf{Q}$ \\
\hline 4 & EQ & $\mathbf{E}$ & SM & MD & D & 28 & UP & E & SM & MD & D & 52 & $\mathrm{AC}$ & E & SM & MD & D \\
\hline 5 & EQ & $E$ & SM & HG & $\mathbf{Q}$ & 29 & UP & E & SM & HG & $\mathbf{Q}$ & 53 & AC & E & SM & HG & Q \\
\hline 6 & EQ & $E$ & SM & HG & D & 30 & UP & $E$ & SM & HG & D & 54 & $A C$ & E & SM & HG & D \\
\hline 7 & EQ & $E$ & AA & LO & $\mathbf{Q}$ & 31 & UP & $E$ & AA & LO & $\mathbf{Q}$ & 55 & AC & E & AA & LO & $\mathbf{Q}$ \\
\hline 8 & EQ & $E$ & AA & LO & D & 32 & UP & $E$ & AA & LO & D & 56 & AC & E & AA & LO & D \\
\hline 9 & EQ & $E$ & AA & MD & $\mathbf{Q}$ & 33 & UP & $E$ & AA & MD & $\mathbf{Q}$ & 57 & AC & E & AA & MD & $\mathbf{Q}$ \\
\hline 10 & EQ & $E$ & AA & MD & D & 34 & UP & $E$ & AA & MD & D & 58 & $A C$ & E & AA & MD & D \\
\hline 11 & EQ & $E$ & AA & HG & $\mathbf{Q}$ & 35 & UP & $E$ & AA & HG & $\mathbf{Q}$ & 59 & $A C$ & E & AA & HG & $\mathbf{Q}$ \\
\hline 12 & EQ & $\mathbf{E}$ & AA & HG & D & 36 & UP & $\mathbf{E}$ & AA & HG & D & 60 & $A C$ & E & AA & HG & D \\
\hline 13 & EQ & $\mathbf{L}$ & SM & LO & $\mathbf{Q}$ & 37 & UP & $\mathbf{L}$ & SM & LO & $\mathbf{Q}$ & 61 & $A C$ & L & SM & LO & $\mathbf{Q}$ \\
\hline 14 & EQ & $\mathbf{L}$ & SM & LO & D & 38 & UP & $\mathbf{L}$ & SM & LO & D & 62 & $A C$ & L & SM & LO & D \\
\hline 15 & EQ & $\mathbf{L}$ & SM & MD & $\mathbf{Q}$ & 39 & UP & $\mathbf{L}$ & SM & MD & $\mathbf{Q}$ & 63 & $A C$ & L & SM & MD & $\mathbf{Q}$ \\
\hline 16 & EQ & L & SM & MD & D & 40 & UP & $\mathbf{L}$ & SM & MD & D & 64 & $A C$ & L & SM & MD & D \\
\hline 17 & EQ & $\mathbf{L}$ & SM & HG & $\mathbf{Q}$ & 41 & UP & $\mathbf{L}$ & SM & HG & $\mathbf{Q}$ & 65 & $A C$ & L & SM & HG & $\mathbf{Q}$ \\
\hline 18 & EQ & L & SM & HG & D & 42 & UP & $\mathbf{L}$ & SM & HG & D & 66 & $A C$ & L & SM & HG & D \\
\hline 19 & EQ & $\mathbf{L}$ & AA & LO & $\mathbf{Q}$ & 43 & UP & $\mathbf{L}$ & AA & LO & $\mathbf{Q}$ & 67 & $A C$ & L & AA & LO & $\mathbf{Q}$ \\
\hline 20 & EQ & $\mathbf{L}$ & AA & 10 & D & 44 & R" & $\mathbf{L}$ & AA & LO & D & 68 & $\mathrm{AC}$ & L & AA & 10 & D \\
\hline 21 & EQ & $\mathbf{L}$ & AA & MD & $\mathbf{Q}$ & 45 & UP & $\mathbf{L}$ & AA & MD & $\mathbf{Q}$ & 69 & $A C$ & $\mathbf{L}$ & AA & MD & $\mathbf{Q}$ \\
\hline 22 & EQ & $\mathbf{L}$ & AA & MD & D & 46 & UP & $\mathbf{L}$ & AA & MD & D & 70 & $A C$ & L & AA & MD & D \\
\hline 23 & EQ & L & A & H & $\mathbf{Q}$ & 4 & U & $\mathbf{L}$ & AA & HG & $\mathbf{Q}$ & 71 & $A C$ & L & AA & HG & $\mathbf{Q}$ \\
\hline 24 & EQ & L & AA & HG & D & 48 & UP & L & AA & HG & D & 72 & $A C$ & L & AA & HG & D \\
\hline
\end{tabular}


The statistical distributions of residuals $\left(\mathrm{vTEC}_{\mathrm{RBMC}}-\mathrm{vTEC}_{\mathrm{IRI}}\right)$ for the 72 classes described above are shown as histograms in Figure 3.16.
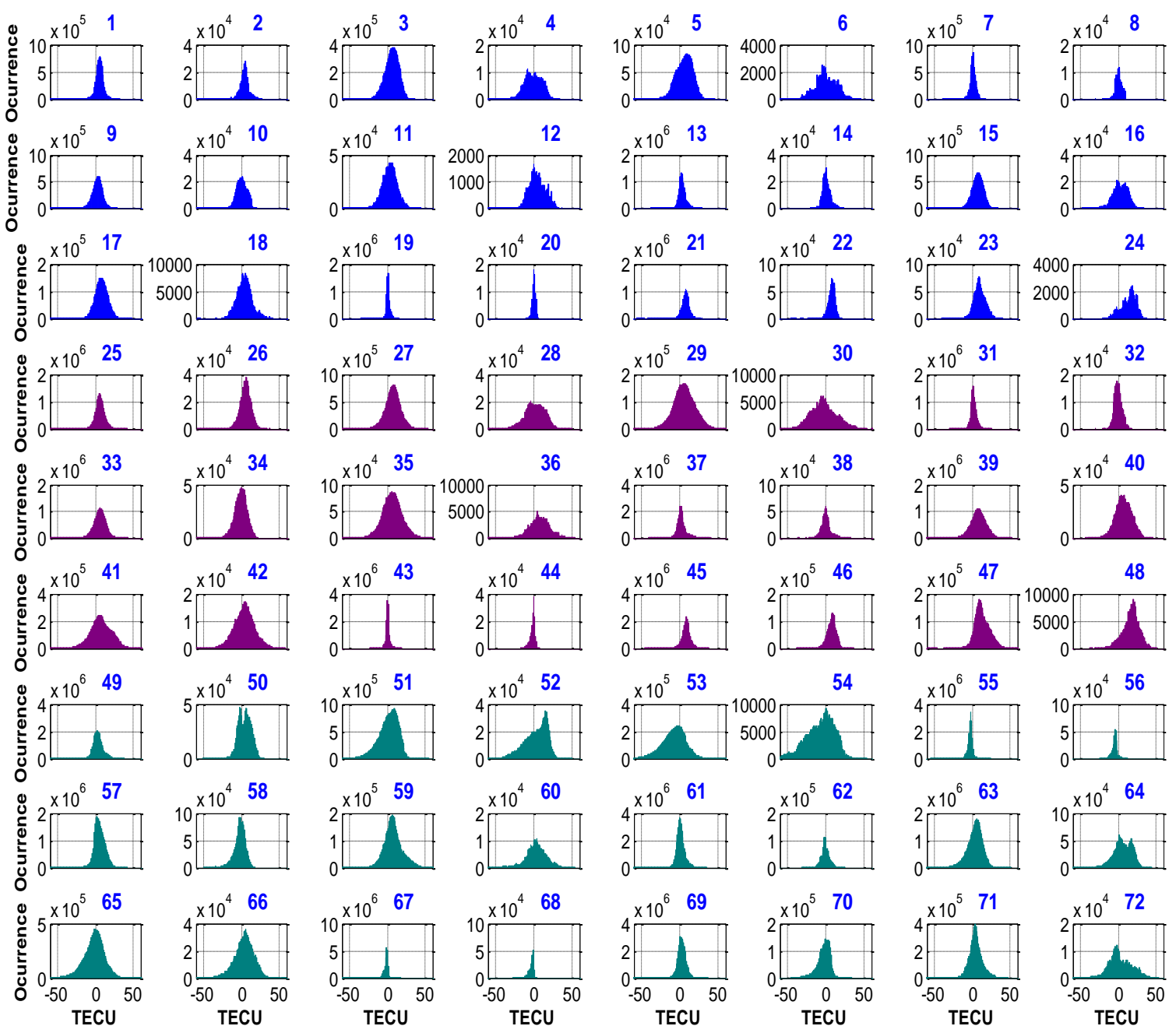

Figure 3.16: Histograms of residuals $\left(\mathrm{vTEC}_{\mathrm{RBMC}}-\mathrm{vTEC}_{\mathrm{IRI}}\right)$.

The histograms estimated from the residuals present varied distributions. Each histogram has a different number of samples. For example class No. 12 (associated with Geomagnetic latitude of IPP: $-4^{\circ}$ to $+4^{\circ}$; Local time: 18:30 LT 21:30 LT; Season: April - August; Solar activity F10.7 > 150; Geomagnetic activity index $\mathrm{Kp}>4$ ) presents a relatively small number of samples $\left(3.6521 \times 10^{4}\right.$ samples). On the other hand, class No. 61 (associated with Geomagnetic latitude of IPP: $-12^{\circ}$ to $-22^{\circ}$; Local time: 21:30 LT - 01:30 LT; Season: September to March; Solar activity index F10.7 < 100; Geomagnetic activity index $\mathrm{Kp} \leq 4)$ presents a relatively large number of samples (3.9351115 $\times 10^{7}$ samples), as shows Figure 3.16. 
Figure 3.17 exhibits histograms and CDF for classes 18, 42 and 66 where the parameter variable is the geomagnetic latitude of IPP: a) $\left(-4^{\circ},+4^{\circ}\right)$, b) $\left(-4^{\circ},-\right.$ $\left.12^{\circ}\right)$ or $\left(+4^{\circ},+12^{\circ}\right)$ and c) $\left(-12^{\circ},-22^{\circ}\right)$ using fixed parameters as local time $(21: 30$ LT - 01:30 LT); EPB season (September - March); solar activity (F10.7>150); geomagnetic activity $(\mathrm{Kp}>4)$.
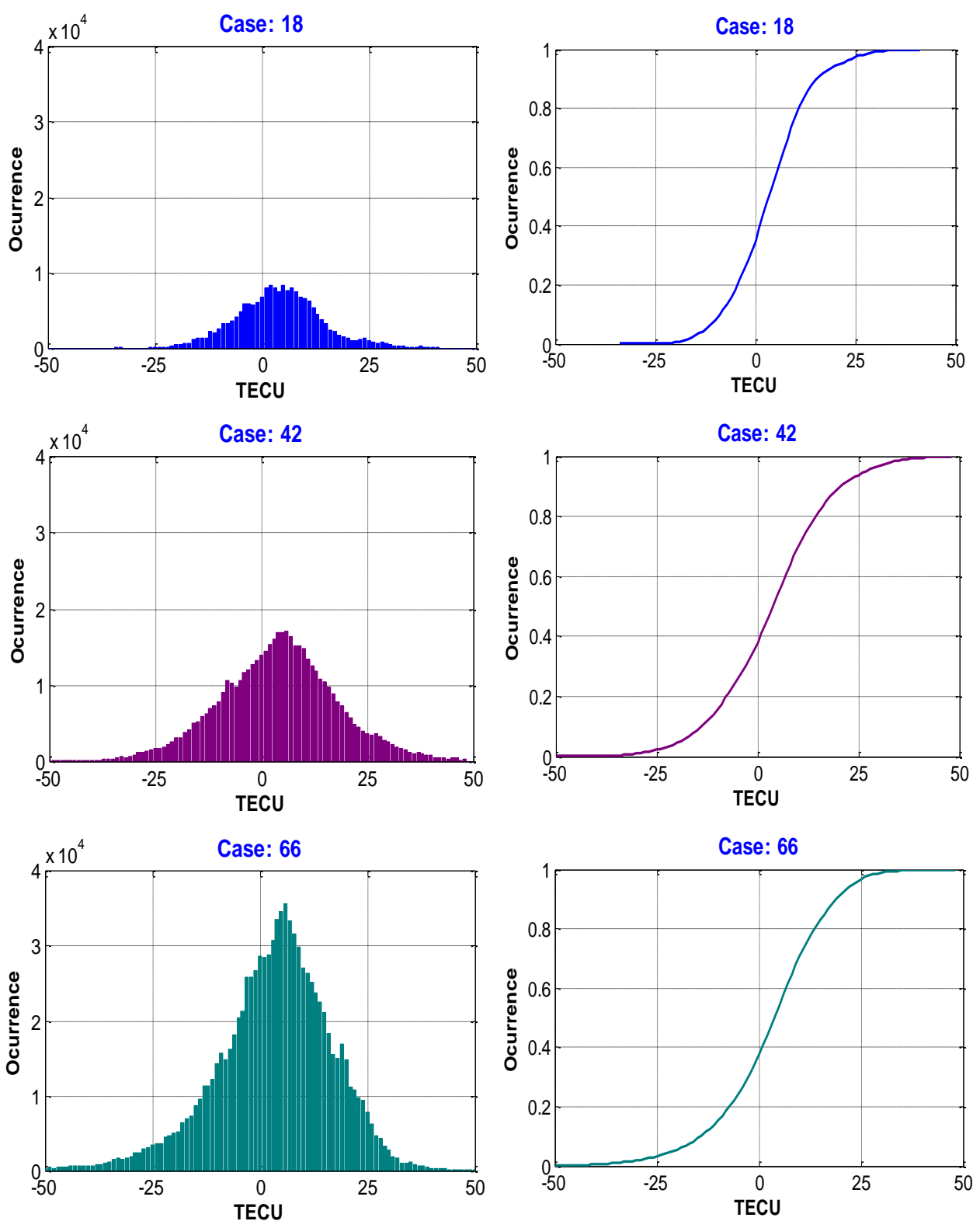

Figure 3.17: Histograms (left) and CDF (right) for the classes 18 [top, geomagnetic latitude of IPP: $\left(-4^{\circ},+4^{\circ}\right)$ ], 42 [middle, geomagnetic latitude of IPP: $\left(-4^{\circ}\right.$,$\left.12^{\circ}\right)$ or $\left.\left(+4^{\circ},+12^{\circ}\right)\right]$ and 66 [bottom, geomagnetic latitude of IPP: $\left.\left(-12^{\circ},-22^{\circ}\right)\right]$. Fixed parameter ranges: local time (21:30 LT-01:30 LT); EPB season (September-March); solar activity (F10.7>150) and geomagnetic activity (Kp >4). 
In Figure 3.17, the three cases show the vertical TEC results performance of the IRI model in comparison to GPS measurements that as result presents a difference $\left(\mathrm{vTEC}_{\mathrm{RBMC}}-\mathrm{vTEC}_{\mathrm{IRI}}\right.$ ) between 2 and 7 TECUs as case of maximum occurrence. Figure 3.17 shows the probabilities peak in the $\left(-12^{\circ},-22^{\circ}\right)$ geomagnetic latitude interval of IPP, being lower in the interval $\left(-4^{\circ},-12^{\circ}\right)$ or $\left(+4^{\circ},+12^{\circ}\right)$ in the number of occurrences.

The maximum residual between $v T E C_{R B M C}-v_{T E C}$ IRI in the geomagnetic latitude interval of IPP $\left(-4^{\circ},-12^{\circ}\right)$ or $\left(+4^{\circ},+12^{\circ}\right)$ is 41 TECUs (Figure 3.17 top, left); in the interval $\left(-4^{\circ},+4^{\circ}\right)$ is 50 TECUs (Figure 3.17 middle, left) and in the interval $\left(-12^{\circ},-22^{\circ}\right)$ is 50 TECUs (Figure 3.17 bottom, left).

In addition, fixing the value of residue (for example, 0 TECUs), the cumulative distribution (Figure 3.17 top, right) shows that the probability that this value is not exceeded is 0.35 for the geomagnetic latitude interval of IPP (-4 ${ }^{\circ}$, $\left.12^{\circ}\right)$ or $\left(+4^{\circ},+12^{\circ}\right) ; 0.38$ for the interval $\left(-4^{\circ},+4^{\circ}\right)$ (Figure 3.17 middle, right) and 0.38 for the interval $\left(-4^{\circ},+4^{\circ}\right)$ (Figure 3.17 bottom, right).

In order to analyze the statistical histograms from Figure 3.16, it was extracted the mean and standard deviation of each distribution as shows Table 3.5. For example, the mean of class No. 48 is 16.81 TECUs, this results reveals that values obtained using IRI model underestimate the values obtained using measurements (Recall class No. 48 is associated to Geomagnetic latitude of IPP: $4^{\circ},-12^{\circ}$ or $+4^{\circ},+12^{\circ}$; Local time: $21: 30$ LT $-01: 30$ LT; Season: April - August; Solar activity (F10.7 index): F10.7>150; Geomag. activity (Kp index): Kp >4).

On the other hand, the mean of the class No. 53 presents -8.11 TECUs, this value reveals that IRI model results overestimate the values obtained using RBMC measurements. (Recall class No. 53 is associated to Geomagnetic latitude of IPP: $-12^{\circ},-22^{\circ}$; Local time: 18:30 LT - 21:30 LT; Season: September - March; Solar activity (F10.7 index): F10.7>150; Geomagetic activity (Kp index): $0<\mathrm{Kp}$ $\leq 4)$. 
Table 3.5: Mean and standard deviation of the 72 statistical distributions (expressed in TECUs).

\begin{tabular}{|c|c|c|c|c|c|c|c|c|}
\hline & Class:1 & Class:2 & Class:3 & Class:4 & Class:5 & Class:6 & Class:7 & Class:8 \\
\hline mean & 5.43 & 3.88 & 5.71 & -1.00 & 5.41 & -0.52 & 0.92 & 0.63 \\
\hline \multirow[t]{2}{*}{ std } & 5.52 & 6.69 & 10.05 & 12.03 & 12.48 & 14.88 & 3.86 & 4.21 \\
\hline & Class:9 & Class: 10 & Class:11 & Class: 12 & Class:13 & Class:14 & Class: 15 & Class:16 \\
\hline mean & 2.44 & 0.33 & 2.74 & 3.09 & 3.56 & 1.01 & 7.22 & 2.95 \\
\hline \multirow[t]{2}{*}{ std } & 7.27 & 7.49 & 10.76 & 13.14 & 4.70 & 5.48 & 7.40 & 9.58 \\
\hline & Class: 17 & Class: 18 & Class: 19 & Class:20 & Class:21 & Class:22 & Class: 23 & Class:24 \\
\hline mean & 8.11 & 3.87 & 1.20 & 0.29 & 7.69 & 7.99 & 9.97 & 13.67 \\
\hline \multirow[t]{2}{*}{ std } & 8.77 & 10.54 & 2.48 & 2.19 & 4.65 & 4.25 & 7.62 & 9.91 \\
\hline & Class:25 & Class:26 & Class:27 & Class:28 & Class:29 & Class:30 & Class:31 & Class:32 \\
\hline mean & 6.07 & 5.30 & 7.25 & 1.57 & 5.80 & -2.56 & 2.12 & -0.06 \\
\hline \multirow[t]{2}{*}{ std } & 6.68 & 7.63 & 10.72 & 13.31 & 14.50 & 17.17 & 4.10 & 4.88 \\
\hline & Class:33 & Class:34 & Class:35 & Class:36 & Class:37 & Class:38 & Class:39 & Class:40 \\
\hline mean & 5.69 & -0.76 & 6.61 & 7.26 & 2.50 & 0.40 & 7.79 & 7.21 \\
\hline \multirow[t]{2}{*}{ std } & 8.47 & 8.32 & 13.18 & 14.17 & 6.34 & 6.50 & 10.32 & 10.90 \\
\hline & Class:41 & Class:42 & Class:43 & Class:44 & Class:45 & Class:46 & Class:47 & Class:48 \\
\hline mean & 7.52 & 4.07 & 0.72 & -1.68 & 8.26 & 7.56 & 13.43 & 16.81 \\
\hline \multirow[t]{2}{*}{ std } & 14.42 & 13.90 & 2.96 & 3.20 & 5.99 & 6.16 & 10.13 & 11.17 \\
\hline & Class:49 & Class:50 & Class:51 & Class:52 & Class:53 & Class:54 & Class:55 & Class:56 \\
\hline mean & 3.61 & 4.50 & 1.27 & 2.43 & -8.11 & -7.41 & -2.48 & -4.42 \\
\hline \multirow[t]{2}{*}{ std } & 6.79 & 8.56 & 13.36 & 15.38 & 16.51 & 18.99 & 3.79 & 3.63 \\
\hline & Class:57 & Class:58 & Class:59 & Class: 60 & Class:61 & Class:62 & Class:63 & Class:64 \\
\hline mean & 5.16 & -2.47 & 7.91 & 2.49 & 1.26 & -0.68 & 3.07 & 6.00 \\
\hline \multirow[t]{2}{*}{ std } & 8.36 & 8.50 & 12.32 & 14.27 & 5.65 & 6.12 & 11.05 & 12.53 \\
\hline & Class:65 & Class:66 & Class:67 & Class:68 & Class:69 & Class:70 & Class:71 & Class:72 \\
\hline mean & -2.21 & 3.12 & -1.76 & -3.26 & 2.67 & -2.71 & 4.76 & 2.57 \\
\hline std & 14.56 & 13.74 & 3.24 & 3.65 & 5.86 & 8.80 & 9.23 & 15.78 \\
\hline
\end{tabular}

The estimations of the VTEC residual associated to the 72 statistical histograms will contribute to estimate the ionospheric delay in the following chapters. 


\section{Estimation of Ionospheric Delay Gradients}

\subsection{Ionospheric Gradient Estimation}

The ionospheric delay gradient is a non-uniform ionospheric structure that can cause errors in differential corrections broadcast to the aircraft using GroundBased Augmentation Systems (GBAS). The present study determines the vertical ionospheric delay gradients using the station-pair $g^{s}(\mathrm{~mm} / \mathrm{km})$ and time-step methods $g^{t}(\mathrm{~mm} / \mathrm{km})$, as shown in equations (4.1) and (4.2), respectively (DattaBarua et al., 2002; Rungraengwajiake et al., 2015).

The station-pair method considers simultaneous ionospheric delays (amount proportional to vTEC) observed in each pair of stations connected to the same satellite, as shown in Figure 4.1.

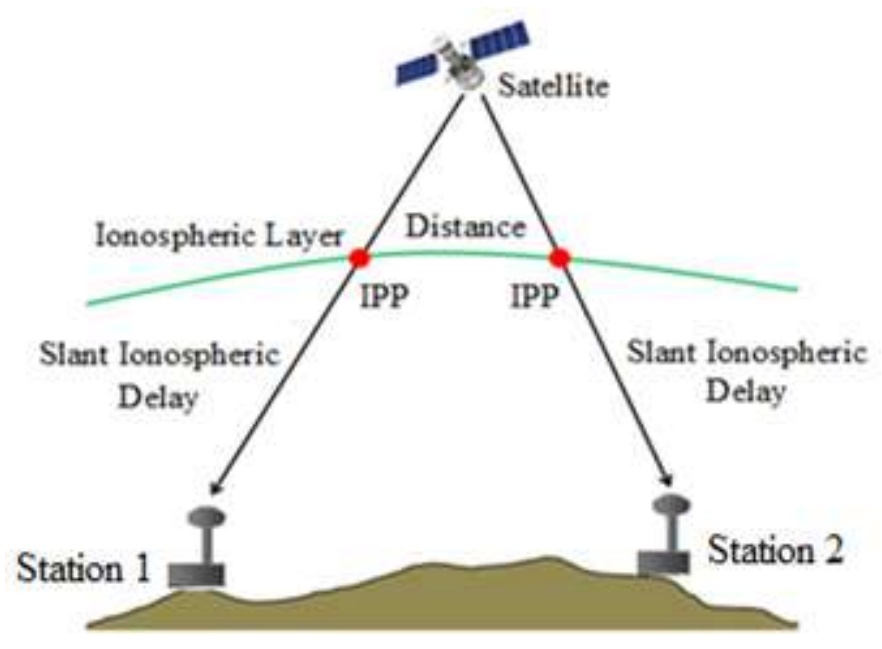

Figure 4.1: Station-Pair method. [Adapted from Pereira et al., 2017]

For a given instant, the estimate of the station-pair ionospheric delay gradient for a given satellite can be represented by the following equation

$$
g^{s}=\frac{\left|I_{r s_{1}}-I_{r s_{2}}\right|}{D I S T_{I P P\left(r s_{1}, r s_{2}\right)}}\left\lceil\frac{\mathrm{mm}}{\mathrm{km}}\right]
$$


where $I_{r s_{1}}$ and $I_{r s_{2}}$ are the values of the vertical ionospheric delays at the IPPs corresponding to satellite $s$; and stations 1 and 2, respectively, and $\operatorname{DIST}_{\operatorname{IPP}\left(r s_{1}, r s_{2}\right)}$ is the distance between the two IPPs. Recall that the ionospheric delay $I$ can be expressed as function of the vTEC using equation (3.2) set as $I=K \cdot T E C / 2 f^{2}$.

The time-step method was developed with the purpose of increasing the sampling of ionospheric gradients and obtaining gradients with small distances than the physical separation of the network stations (Datta-Barua et al., 2002, Pereira et al., 2017). The method involves a single satellite and a single station, as well as ionospheric delays (associated to vTEC) at consecutive time instants, as shown in Figure 4.2.

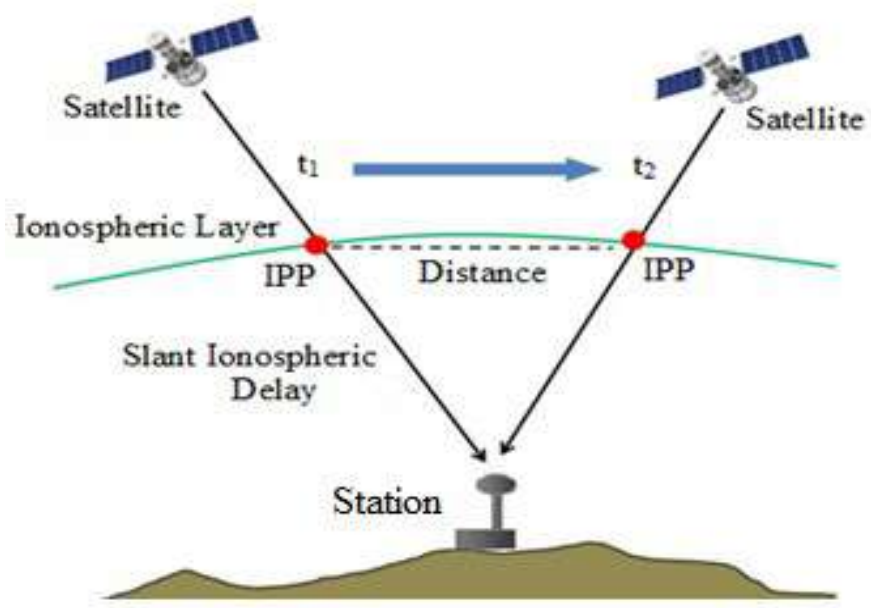

Figure 4.2: Time-Step method. [Adapted from Pereira et al., 2017]

The time-step ionospheric delay between the satellite and the receiver station at a time $t_{2}$ is subtracted from the delay of the same pair at time $t_{1}$. The gradient is then represented by the following equation

$$
g^{t}=\frac{\left|I_{r t_{1}}-I_{r t_{2}}\right|}{D I S T_{I P P\left(r t_{1}, r t_{2}\right)}}\left[\frac{\mathrm{mm}}{\mathrm{km}}\right]
$$

where $I_{r t_{1}}$ and $I_{r t_{2}}$ are vertical ionospheric delay values for the receiving station and the satellite at time instants $t_{1}$ and $t_{2}$, respectively. $D I S T_{I P P}\left(r t_{1}, r t_{2}\right)$ is the distance between the two IPPs referring to satellite positions at these instants of time. 


\subsection{Results of the Vertical Ionospheric Delay Gradient Using the Time-Step Method}

This contribution presents the statistical distributions of vertical ionospheric delay gradients estimated using the time-step method estimated every 15 seconds (sampling rate) based on data from Imperatriz (IMPZ), Brasília (BRAZ) and Rio de Janeiro (RIOD) receiver stations during the years 2002, 2008, and 2013. These stations were selected because are located in different geomagnetic latitudes. The geodetic position of each receiver is described in Table 3.2. Recall that the vertical TEC was estimated using the procedure described in Chapter 3.

The complementary cumulative distribution function (CCDF) in logarithmic scale using data corresponding to the period between 01 January 2002 and 31 December 2002 is shown in Figure 4.3. It should be remarked that the maximum gradients, not shown in Figure 4.3, are equal to $372.52 \mathrm{~mm} / \mathrm{km}$ (IMPZ), 488.78 $\mathrm{mm} / \mathrm{km}$ (BRAZ), and $490.57 \mathrm{~mm} / \mathrm{km}$ (RIOD).

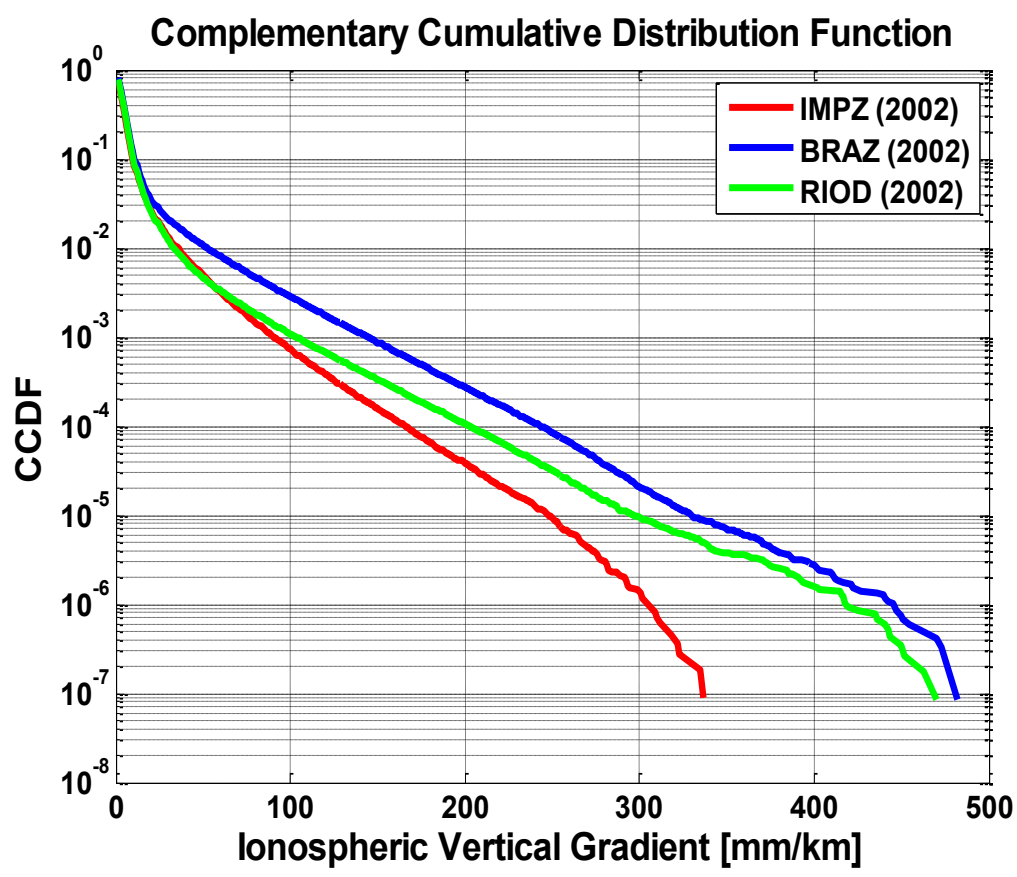

Figure 4.3: CCDF of the vertical ionospheric delay gradients estimated using the time-step method based on data from IMPZ, BRAZ and RIOD stations in 2002.

For the solar maximum year 2002, the results from the IMPZ station presents relatively low percentages of large ionospheric gradients in comparison with those associated with the BRAZ and RIOD stations as shows Figure 4.3. It should be 
remarked that IMPZ is located near to the geomagnetic equator. Also, the BRAZ station reveals higher percentages of vertical gradients than the other stations. For example, the probability to get a gradient corresponding to $300 \mathrm{~mm} / \mathrm{km}$ is $1.5 \times 10^{-6}$ (IMPZ), $9.7 \times 10^{-6}$ (RIOD) and $2 \times 10^{-5}$ (BRAZ).

Similarly, Figure 4.4 presents the CCDF of gradients estimated during 2008. The maximum gradients, not shown in Figure 4.4, are equal to $314.09 \mathrm{~mm} / \mathrm{km}$ (IMPZ), $402.87 \mathrm{~mm} / \mathrm{km}$ (BRAZ), and $113.49 \mathrm{~mm} / \mathrm{km}$ (RIOD).

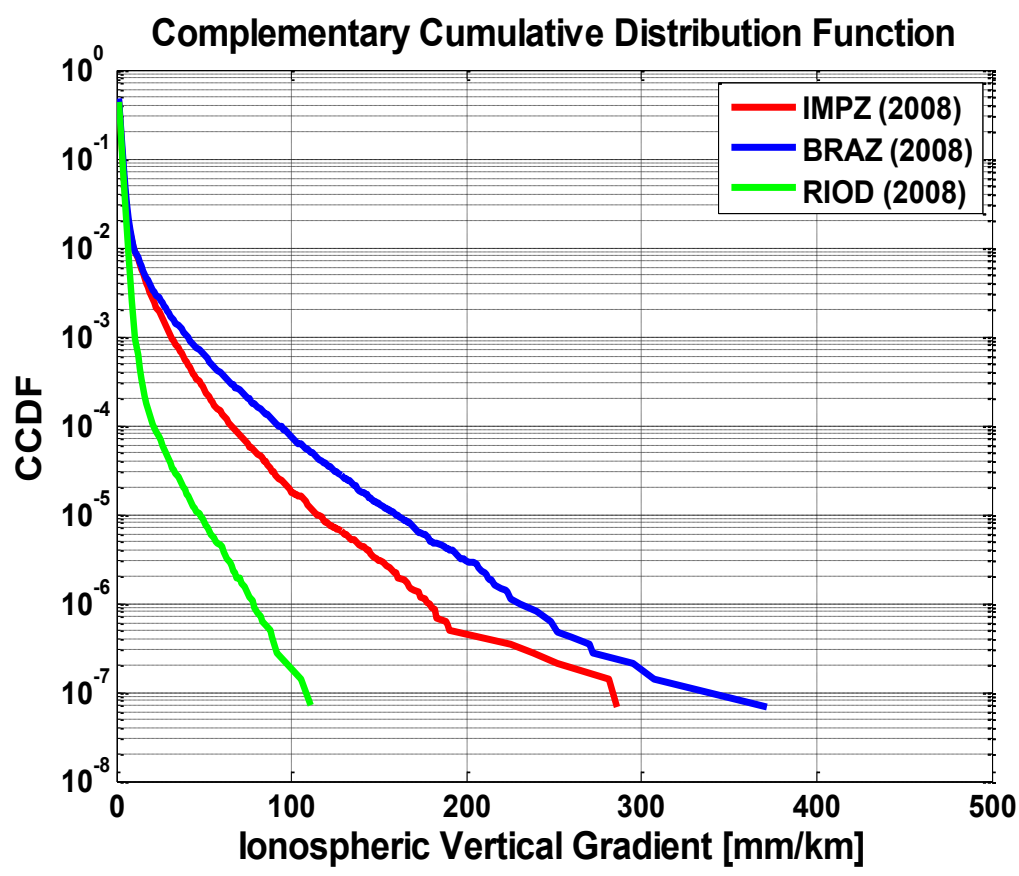

Figure 4.4: CCDF of the vertical ionospheric delay gradient estimated using the time-step method based on data from IMPZ, BRAZ and RIOD stations in 2008.

For the solar minimum year 2008, RIOD station presents the lowest percentages of ionospheric gradients in comparison with those associated with the IMPZ and BRAZ stations as is shown in Figure 4.4. The most remarkable is that IMPZ and BRAZ stations are located near to the crest of EIA. Again, the BRAZ station reveals large percentages of vertical gradients than the other stations.

The CCDFs of gradients estimated during 2013 are shown in Figure 4.5. The maximum gradient, not shown in Figure 4.5, are equal to $588.79 \mathrm{~mm} / \mathrm{km}$ (IMPZ), $974.23 \mathrm{~mm} / \mathrm{km}$ (BRAZ) and $970.71 \mathrm{~mm} / \mathrm{km}$ (RIOD). 


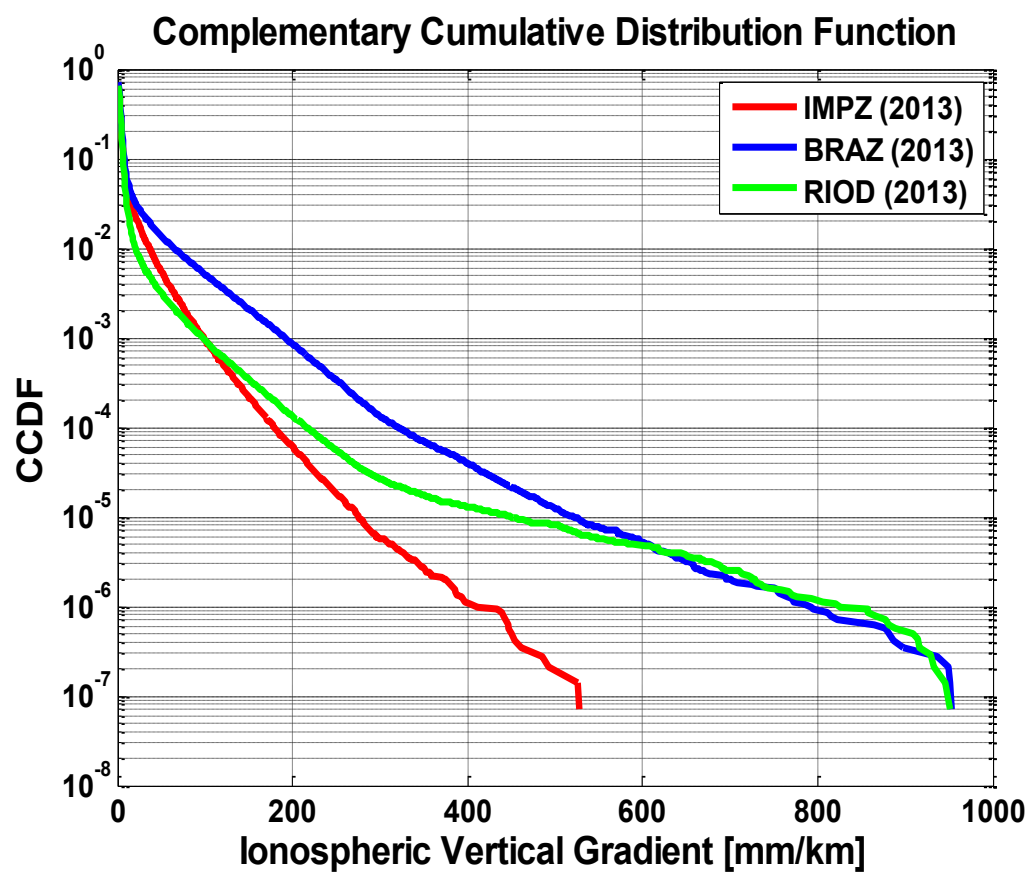

Figure 4.5: CCDF of the vertical ionospheric delay gradient estimated using the time-step method based on data from IMPZ, BRAZ and RIOD stations in 2013.

Figure 4.5 shows that the results estimated for IMPZ station present low percentages of large vertical gradients in comparison to those associated with the BRAZ and RIOD stations. Also, the results of BRAZ station reveal higher percentages of large vertical gradients than the other stations from in the range 0 $\mathrm{mm} / \mathrm{km}$ to $600 \mathrm{~mm} / \mathrm{km}$. However, the BRAZ and RIOD stations present similar percentages of vertical gradients from $600 \mathrm{~mm} / \mathrm{km}$ to their respective maximum values. For example, the probability to get a gradient corresponding to $400 \mathrm{~mm} / \mathrm{km}$ is $1.2 \times 10^{-6}$ (IMPZ), $1.4 \times 10^{-5}$ (RIOD) and $3.9 \times 10^{-5}$ (BRAZ).

\subsection{Daily Maximum Vertical lonospheric Delay Gradient Using the Time-Step Method}

It is relevant to assess the maximum gradients along the time to examine the days with high solar activity. For this reason, the time variation of the daily maximum vertical ionospheric gradient (VIG) using the time-step method for the IMPZ (red), BRAZ (blue) and RIOD (green) stations for the years 2002, 2008 and 2013 are shown in each panel of Figure 4.6. The absolute maximum values of VIG for the three stations and years are organized in Table 4.1. 

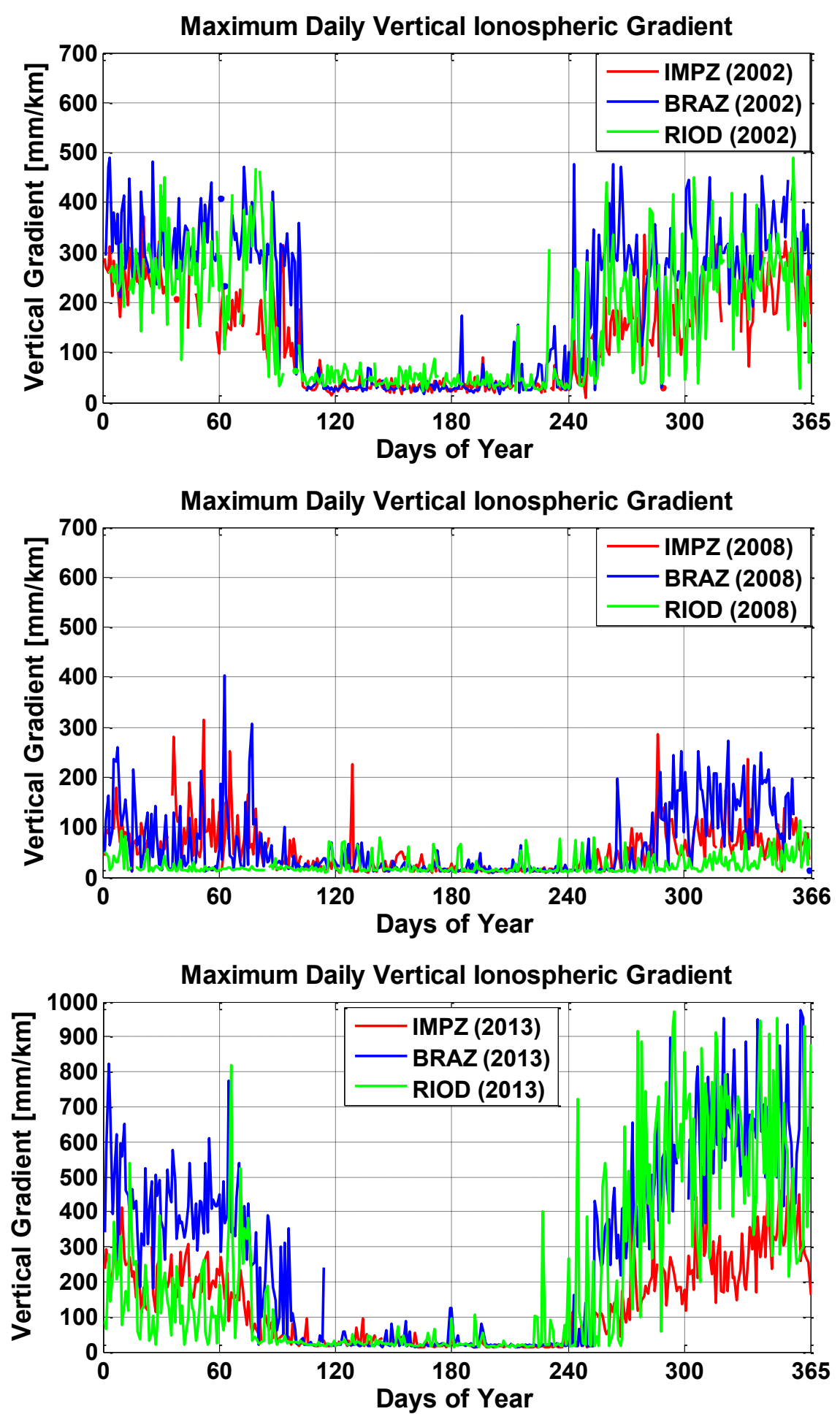

Figure 4.6: Time variation of the daily maximum vertical ionospheric delay gradient for the IMPZ (red), BRAZ (blue) and RIOD (green) stations in 2002 (top), 2008 (middle) and 2013 (bottom). 
Table 4.1: Absolute maximum values of VIG in 2002, 2008, and 2013.

\begin{tabular}{|c|c|c|c|c|}
\hline \multirow{3}{*}{ Year } & Station & $\begin{array}{c}\text { Maximum VIG } \\
{[\mathbf{m m} / \mathbf{k m}]}\end{array}$ & DOY & $\begin{array}{c}\text { Index } \\
\text { F10.7 }\end{array}$ \\
\hline \multirow{3}{*}{$\mathbf{2 0 0 2}$} & IMPZ & 372.52 & 021 & 217.5 \\
\cline { 2 - 5 } & BRAZ & 488.78 & 004 & 211.0 \\
\cline { 2 - 5 } & RIOD & 490.57 & 356 & 166.4 \\
\hline \multirow{3}{*}{$\mathbf{2 0 0 8}$} & IMPZ & 314.09 & 052 & 70.3 \\
\cline { 2 - 5 } & BRAZ & 402.87 & 063 & 67.3 \\
\cline { 2 - 5 } & RIOD & 113.49 & 360 & 67.0 \\
\hline \multirow{3}{*}{$\mathbf{2 0 1 3}$} & IMPZ & 588.79 & 273 & 105.2 \\
\cline { 2 - 5 } & BRAZ & 974.23 & 360 & 120.6 \\
\cline { 2 - 5 } & RIOD & 970.71 & 295 & 144.8 \\
\hline
\end{tabular}

Each subplot of Figure 4.6 shows that the smallest values of daily maximum VIG occur between the days 100 and 240 (April to August, covering the winter months of the southern hemisphere), as expected. In addition, the BRAZ station present large values of maximum vertical gradients in a large number of days between September to March corresponding to the years 2002, 2008 and 2013. However, IMPZ station is close to equatorial geomagnetic latitude and present low gradients in comparison to BRAZ and RIOD stations in 2002 and 2013 (high solar activity) as is shown in Figure 4.6. During the solar minimum year, RIOD station reveals low ionospheric gradients.

\subsection{Results of the Vertical Ionospheric Delay Gradient Using the Station-Pair Method}

The vertical ionospheric delay (VID) and vertical ionospheric delay gradient (VIG) were estimated using measurements of all observed GPS satellites obtained each 15 seconds (sampling rate) from the RBMC station pairs: SSA1 - SAVO, ONRJ - RIOD and CEEU- CEFT, located in different magnetic dip latitudes as is shown in Figure 4.7 and summarized in Table 4.2. 


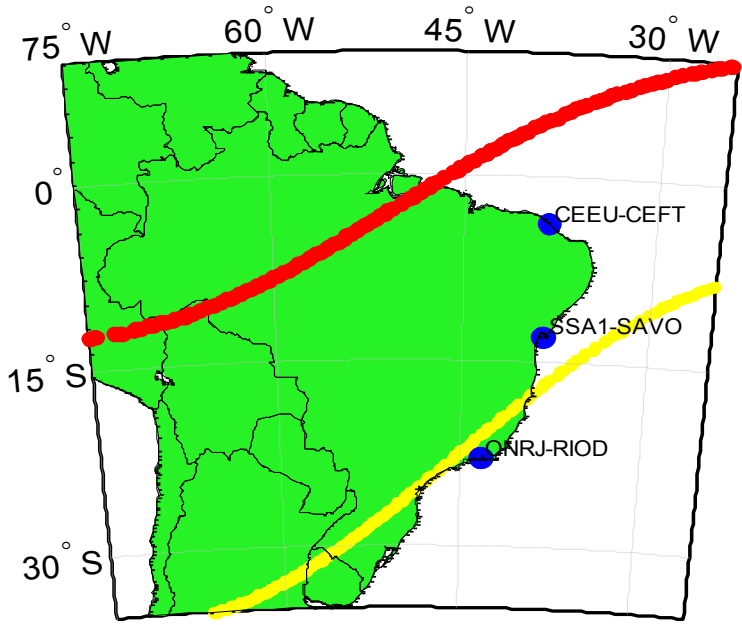

Figure 4.7: Positions of the ground-based RBMC station-pairs in 2013 (blue dots).

Table 4.2: Description of station pairs.

\begin{tabular}{|c|c|c|c|c|c|}
\hline $\begin{array}{c}\text { Station } \\
\text { Pairs }\end{array}$ & Location & $\begin{array}{l}\text { Geographic } \\
\text { Coordinates }\end{array}$ & $\begin{array}{c}\text { Dip } \\
\text { Latitude }\end{array}$ & $\begin{array}{c}\text { Distance } \\
(\mathbf{k m})\end{array}$ & $\begin{array}{c}\text { Azimuth } \\
\text { between } \\
\text { stations }\end{array}$ \\
\hline CEEU- CEFT & $\begin{array}{l}\text { Fortaleza, } \\
\text { Brazil }\end{array}$ & $\begin{array}{ll}3.87^{\circ} \mathrm{S} & 38.42^{\circ} \mathrm{W} \\
3.70^{\circ} \mathrm{S} & 38.47^{\circ} \mathrm{W}\end{array}$ & $\begin{array}{l}7.34^{\circ} \mathrm{S} \\
7.15^{\circ} \mathrm{S}\end{array}$ & 19.17 & $164.07^{\circ}$ \\
\hline SSA1 - SAVO & $\begin{array}{l}\text { Salvador, } \\
\text { Brazil }\end{array}$ & $\begin{array}{ll}12.98^{\circ} \mathrm{S} & 38.52^{\circ} \mathrm{W} \\
12.93^{\circ} \mathrm{S} & 38.43^{\circ} \mathrm{W}\end{array}$ & $\begin{array}{l}15.47^{\circ} \mathrm{S} \\
15.48^{\circ} \mathrm{S}\end{array}$ & 9.96 & $66.52^{\circ}$ \\
\hline ONRJ - RIOD & $\begin{array}{c}\text { Rio de Janeiro, } \\
\text { Brazil }\end{array}$ & $\begin{array}{ll}22.90^{\circ} \mathrm{S} & 43.22^{\circ} \mathrm{W} \\
22.82^{\circ} \mathrm{S} & 43.30^{\circ} \mathrm{W}\end{array}$ & $\begin{array}{l}20.82^{\circ} \mathrm{S} \\
20.83^{\circ} \mathrm{S}\end{array}$ & 12.04 & $135.72^{\circ}$ \\
\hline
\end{tabular}

Each plot of Figure 4.8 shows the results of the vertical ionospheric delay estimations using measurements of each combination of a GPS satellite with the SSA1 (represented by a different line color); and SAVO stations (represented by a red line color) on 10 November 2013.

Figure 4.8 reveals the magnitude of the vertical delay due to the ionosphere. For example, satellite 2 presents a vertical delay between 5 and 15 meters as is depicted in the vertical axis for both stations, but SAVO station (red line) presents a large delay in comparison to SSA1 station (color line) during the respective epoch. 

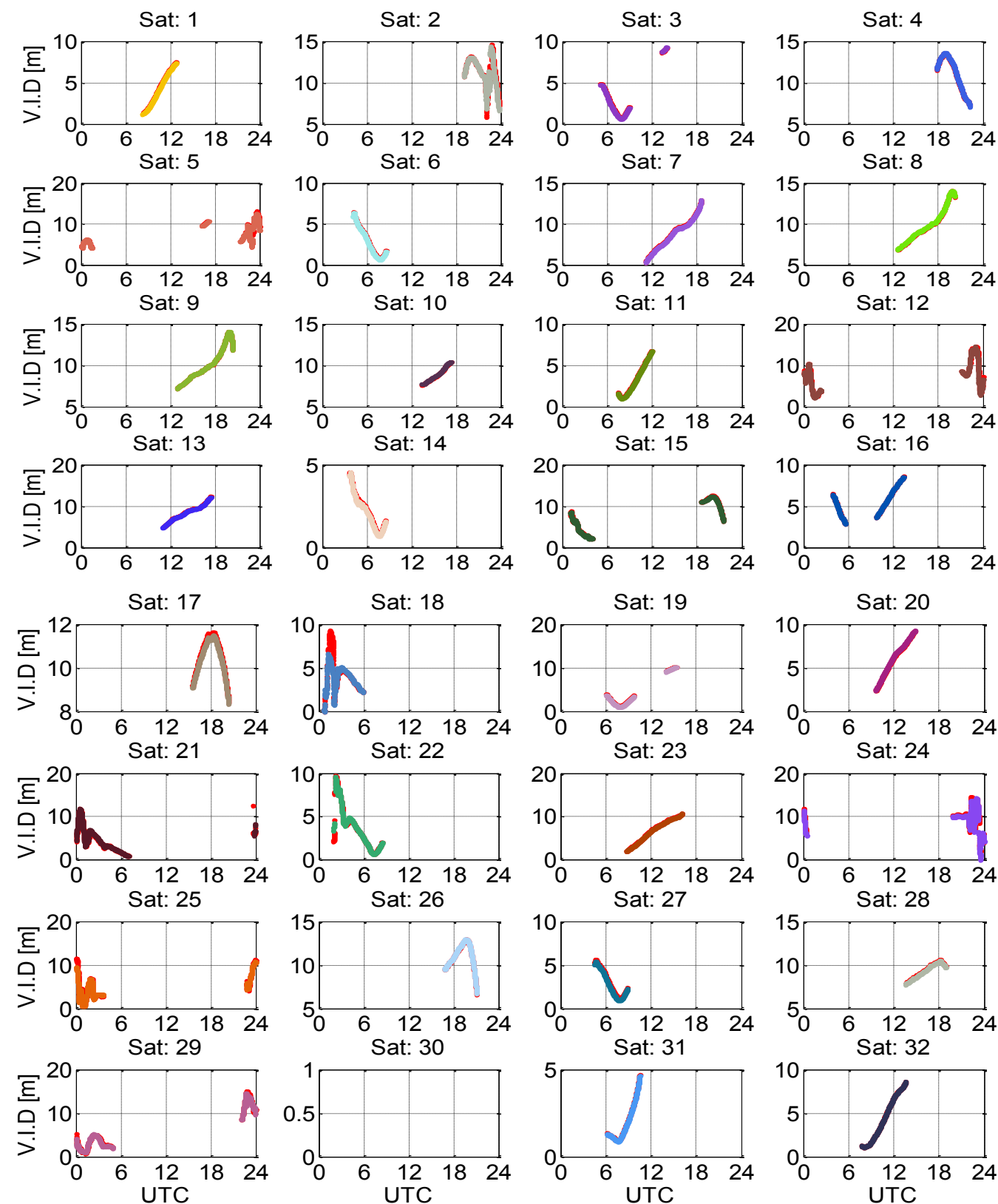

Figure 4.8: Vertical ionospheric delays computed for each GPS satellite and station SSA1; and SAVO on 10 November 2013.

The estimated vertical ionospheric delay for each station - GPS satellite and the distance between the corresponding IPP were used to compute the gradient. Figure 4.9 shows the vertical ionospheric delay gradient estimated using measurements of each GPS satellite and the pair of SSA1 and SAVO stations on 10 November 2013. 

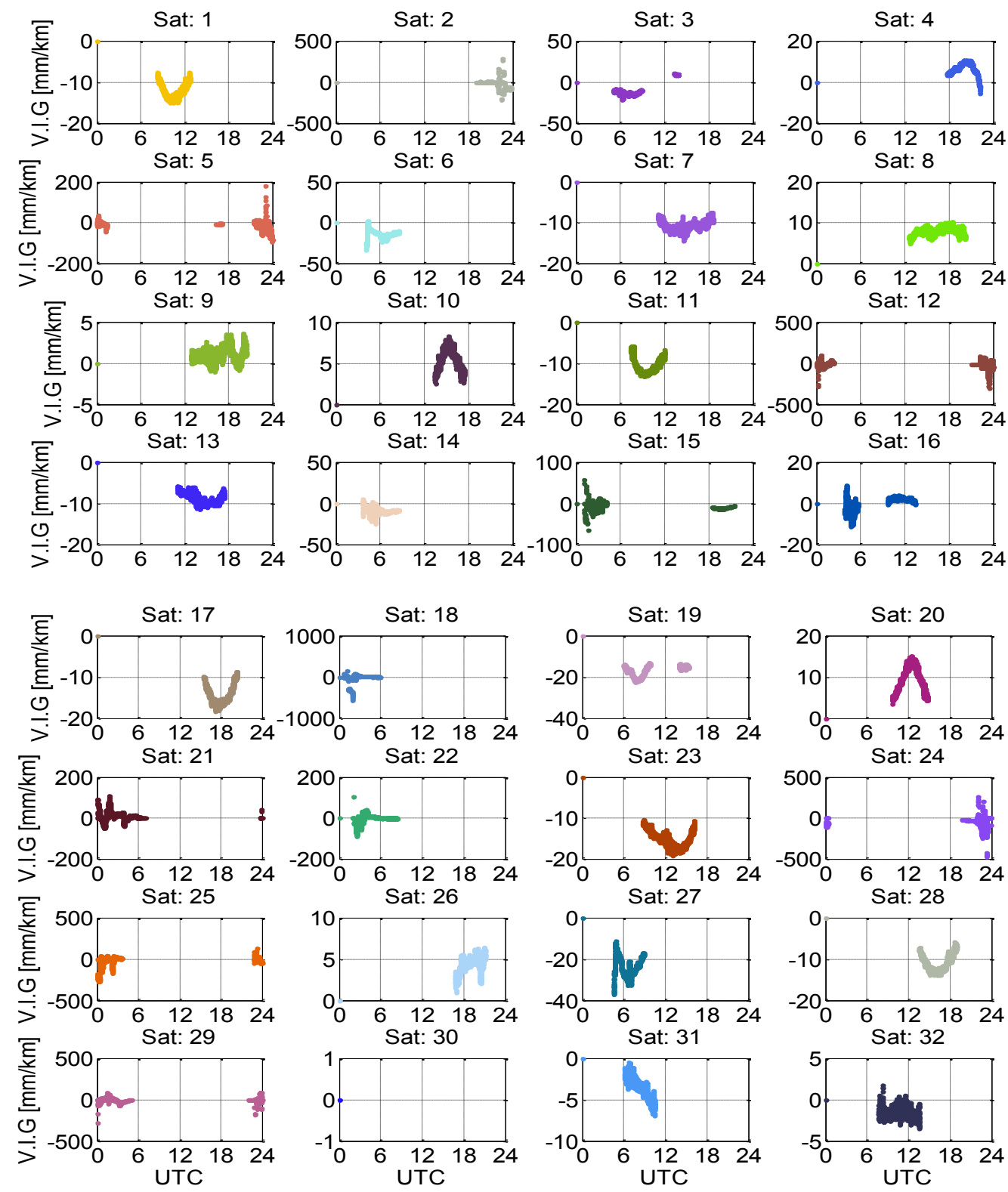

Figure 4.9: Vertical ionospheric delay gradient computed betweenthe two stations

SAVO and SSA1 on 10 November 2013.

All of the significant gradients are observed during the post-sunset hours between 21:00 and 05:00 (UTC). Examples of large gradients are seen in the plots associated with GPS satellites 2, 5, 12, 18, 24, 25, and 29. The predominance of those gradients occurred between 00:00 - 04:00 (UTC). The maximum value of vertical gradient is $571.2 \mathrm{~mm} / \mathrm{km}$ at 01:57:30 UTC, for satellite 18 as is shown in Figure 4.9 and 4.10.

One example of large variation of vertical delay measured by the two stations, shown in Figure 4.9, is highlighted in Figure 4.10. This Figure shows the measured 
vertical ionospheric delay in satellite 18 signals, as measured from by the two stations SSA1 and SAVO (located $9.96 \mathrm{~km}$ away from each other). The vertical delay shows features that could be associated with Equatorial Plasma Bubbles (EPBs) effects on the GPS estimates of ionospheric delays.
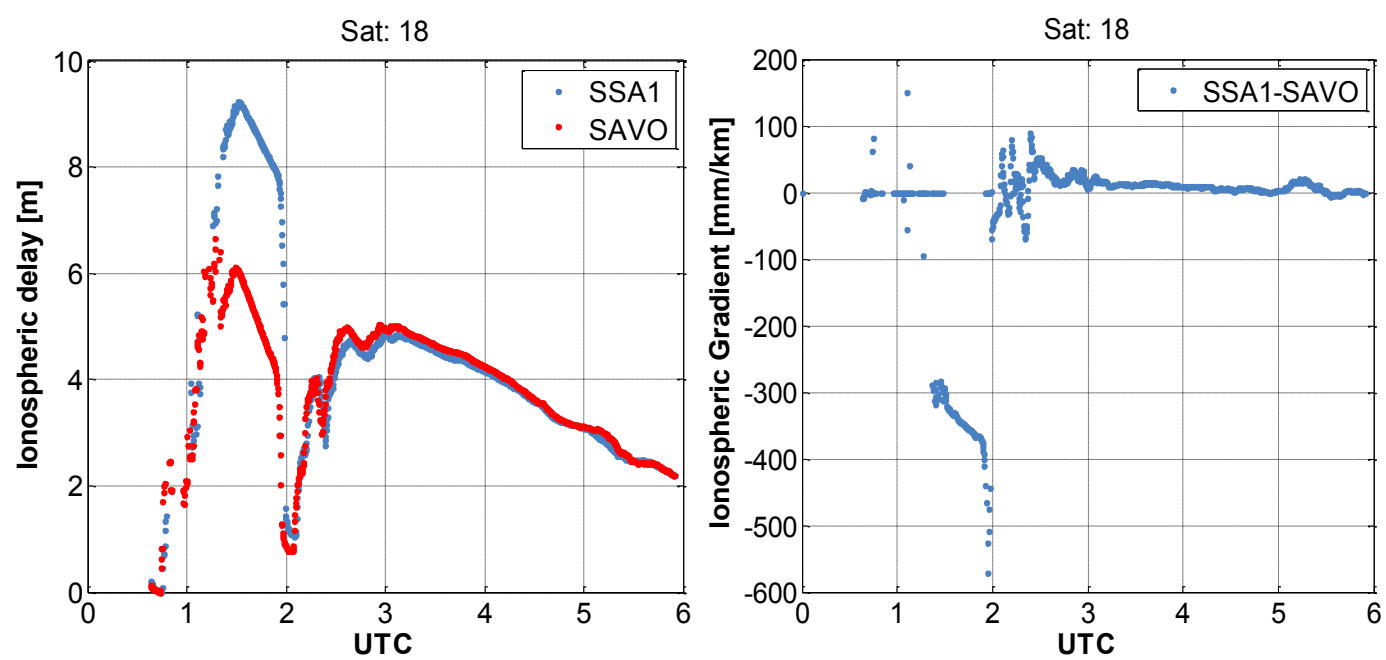

Figure 4.10: Estimated vertical ionospheric delay (Left); corresponding vertical ionospheric delay gradient, associated with satellite 18 and the two stations SAVO and SSA1 on 10 November 2013 (Right).

Table 4.3 contains the list of significant gradients observed for the pair SAVO-SSA1 estimated by (Mirus, 2015) using the LTIAM software in 2013 and 2014. The Table specifies the DOY, satellite number, elevation, azimuth, and the observation hour of the ionospheric gradient.

Table 4.3: Largest slant ionospheric gradients estimated for the pair: SAVO-SSA1 stations in 2013 and 2014. [Reproduced from Mirus, 2015]

\begin{tabular}{|c|c|c|c|c|c|c|}
\hline $\begin{array}{c}\text { DOY- } \\
\text { Year }\end{array}$ & Stations & $\begin{array}{c}\text { Azimuth } \\
\left({ }^{\circ}\right)\end{array}$ & $\begin{array}{c}\text { Sat. } \\
\text { Hour } \\
(\mathbf{U T C})\end{array}$ & $\begin{array}{c}\text { Slope } \\
(\mathbf{m m} / \mathbf{k m})\end{array}$ & $\begin{array}{c}\text { Elevation } \\
\left({ }^{\circ}\right)\end{array}$ \\
\hline $314-2013$ & SAVO-SSA1 & 325.42 & 18 & $1: 57: 30$ & 641.2 & 55.06 \\
\hline $310-2013$ & SAVO-SSA1 & 336.62 & 18 & $1: 11: 30$ & 596.4 & 27.09 \\
\hline $319-2013$ & SAVO-SSA1 & 339.82 & 24 & $22: 51: 00$ & 548.0 & 46.38 \\
\hline $365-2013$ & SAVO-SSA1 & 177.68 & 21 & $23: 51: 30$ & 501.2 & 38.25 \\
\hline $055-2014$ & SAVO-SSA1 & 315.10 & 3 & $1: 13: 15$ & 562.6 & 27.33 \\
\hline $056-2014$ & SAVO-SSA1 & 337.33 & 27 & $1: 05: 30$ & 553.3 & 29.27 \\
\hline $065-2014$ & SAVO-SSA1 & 304.49 & 11 & $2: 19: 45$ & 510.2 & 49.23 \\
\hline
\end{tabular}


The analysis of the threatening gradients made by (Mirus, 2015) revealed that all of the larger gradients identified through this process occurred during post sunset hours and in conjunction with post-sunset ionospheric depletion. The depletion formed post sunset. Additionally, these depletions are considered a key source of scintillation on the GPS signal, hence it can be concluded that there may exist a high degree of correlation between depth of the depletions and scintillations to the threatening gradients for GBAS.

Figure 4.11 and 4.12 display the CCDFs of the vertical gradients associated with the station pairs listed in Table 4.2 for the years 2008 (CEEU- CEFT pair is not available) and 2013. Figure 4.11 reveals that, for the solar-minimum year 2008, the SSA1-SAVO station pair presents high percentages of large ionospheric gradients between 0 and $100 \mathrm{~mm} / \mathrm{km}$, in comparison to those associated estimated by the ONRJ-RIOD station pair. It should be remarked that the maximum gradients, not shown in Figure 4.11, are equal to $284.32 \mathrm{~mm} / \mathrm{km}$ (ONRJ-RIOD), and 140.10 $\mathrm{mm} / \mathrm{km}(\mathrm{SSA} 1-\mathrm{SAVO})$.

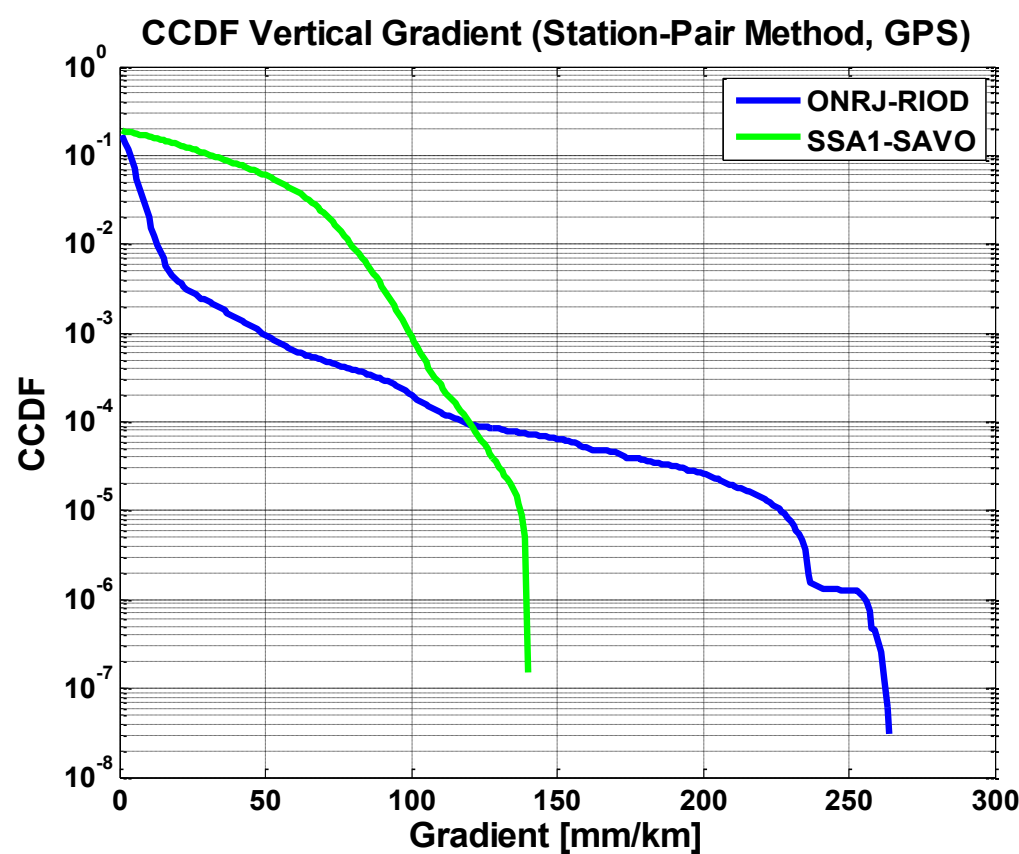

Figure 4.11: CCDFs of the vertical ionospheric delay gradients estimated by the station-pair method based on data from the combinations of stations: ONRJ-RIOD (blue) and SSA1-SAVO (green) in 2008.

On the other hand, for the solar-maximum year 2013, the ONRJ-RIOD station pair presents high percentage of large ionospheric gradients, in comparison those 
associated with the CEEU-CEFT and SSA1-SAVO station pairs as is shown in Figure 4.12. The ONRJ-RIOD station pair is located in the poleward side of the southern crest of the EIA. The CEEU-CEFT station present small percentage of large gradients, it should be remembered that CEEU-CEFT station pair is located slightly nearer to the geomagnetic equator than the other two. It should be remarked that the maximum gradients, not shown in Figure 4.12, are equal to $939.90 \mathrm{~mm} / \mathrm{km}$ (ONRJ-RIOD), $334.40 \mathrm{~mm} / \mathrm{km}$ (CEEU-CEFT) and $908.33 \mathrm{~mm} / \mathrm{km}$ (SSA1-SAVO).

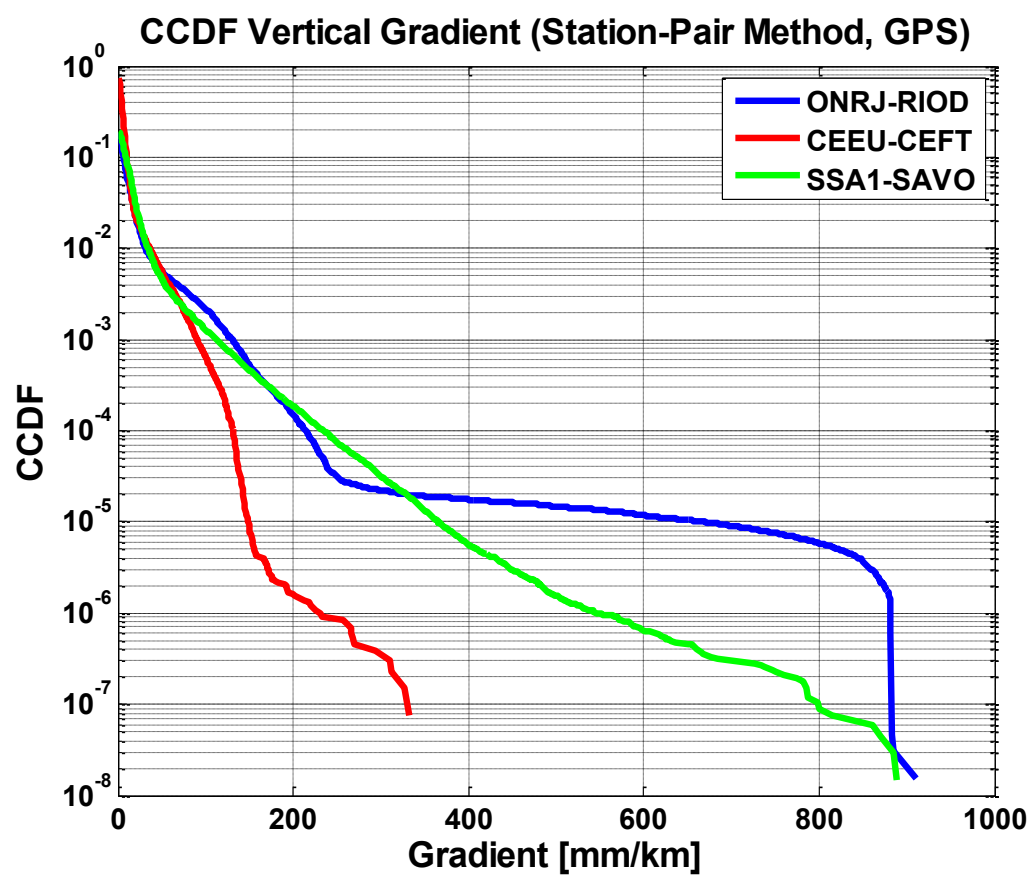

Figure 4.12: CCDFs of the vertical ionospheric delay estimated by the station-pair method based on data from the combinations of stations: ONRJ-RIOD (blue), CEEU-CEFT (red), and SSA1-SAVO (green) in 2013.

Figure 4.13 shows the daily values of the mean and standard deviation of the vertical ionospheric gradient (VIG) estimated from all GPS satellites using the Station-pair method for the pairs: SSA1-SAVO and RIOD-ONRJ stations in 2008. As the figure shows, most of the mean values of the VIG are between 8 and 10 $\mathrm{mm} / \mathrm{km}$ for SSA1-SAVO pair; between 1 and $3 \mathrm{~mm} / \mathrm{km}$ for RIOD-ONRJ pair. In addition, most standard deviation values of the VIG ( $\left.\sigma_{\text {vert_iono_grad }}\right)$ are between 10 and $14 \mathrm{~mm} / \mathrm{km}$ for SSA1-SAVO pair; between 2 and $6 \mathrm{~mm} / \mathrm{km}$ for RIOD-ONRJ pair. 

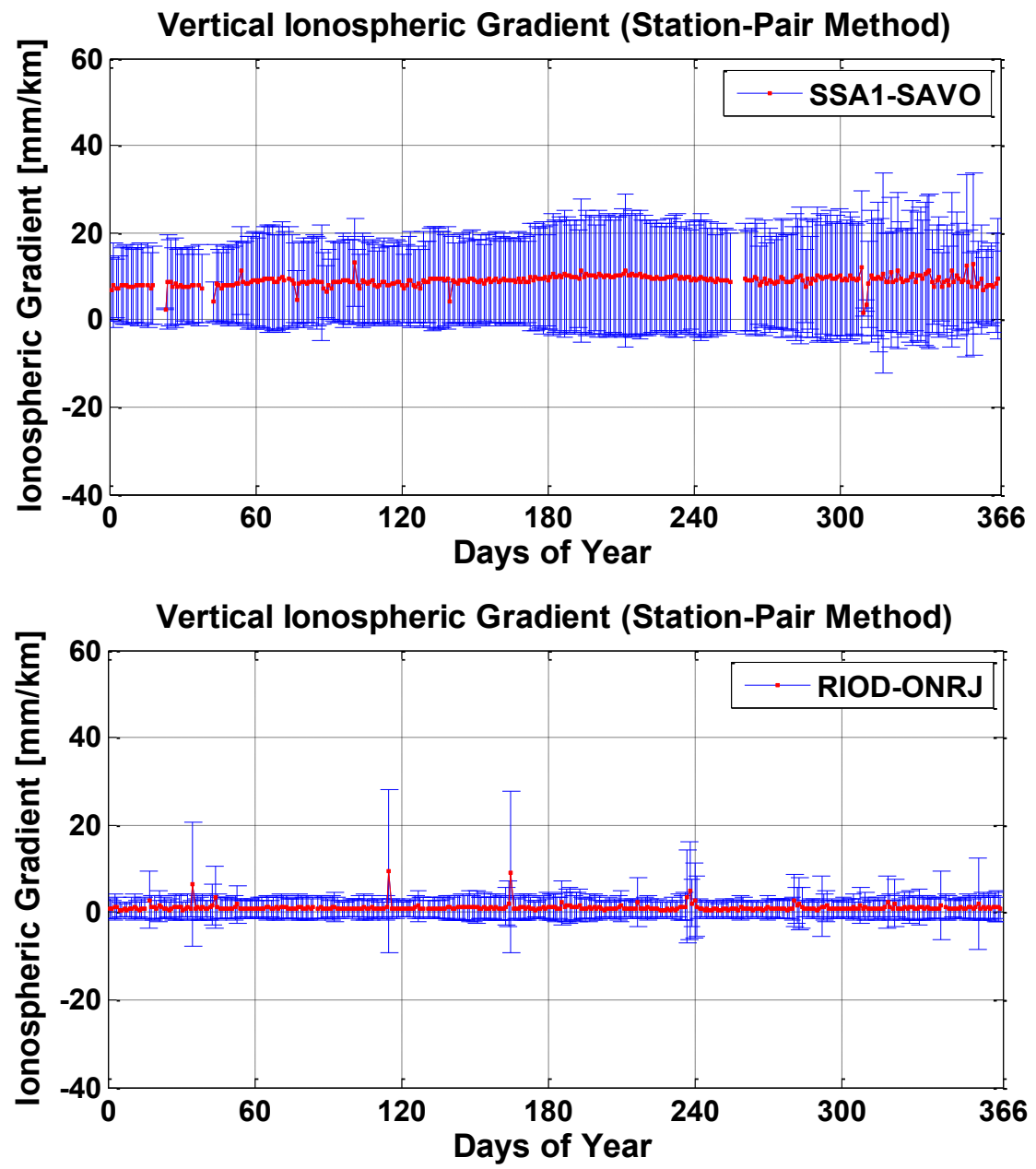

Figure 4.13: Daily values of the mean and standard deviation of VIG estimated by the station-pair method computed for SSA1 and SAVO stations (top); RIOD and ONRJ stations (bottom) in 2008.

Figure 4.14 shows the daily values of the mean and standard deviation of the vertical ionospheric delay gradient estimated using the Station-pair method for the pairs: CEEU-CEFT, SSA1-SAVO and RIOD-ONRJ stations in 2013. As the figure shows, most of the mean values of the VIG are between 2 and $4 \mathrm{~mm} / \mathrm{km}$ for SSA1SAVO and RIOD-ONRJ pairs and between 2 and $8 \mathrm{~mm} / \mathrm{km}$ for CEEU-CEFT pair.

In the Figure 4.14, most of the standard deviation values of the VIG $\left(\sigma_{\text {vert_iono_grad }}\right)$ are between 4 and $14 \mathrm{~mm} / \mathrm{km}$ for SSA1-SAVO pair; between 4 and $16 \mathrm{~mm} / \mathrm{km}$ for RIOD-ONRJ pair and between 2 and $10 \mathrm{~mm} / \mathrm{km}$ for CEEU-CEFT pair. It should be remarked that the maximum VIG ( $\left.\sigma_{\text {vert_iono_grad }}\right)$ occur between the days 1 and 100; 240 and 365 (aproximatly January to March and September to December, covering the summer and spring months of the southern hemisphere). 

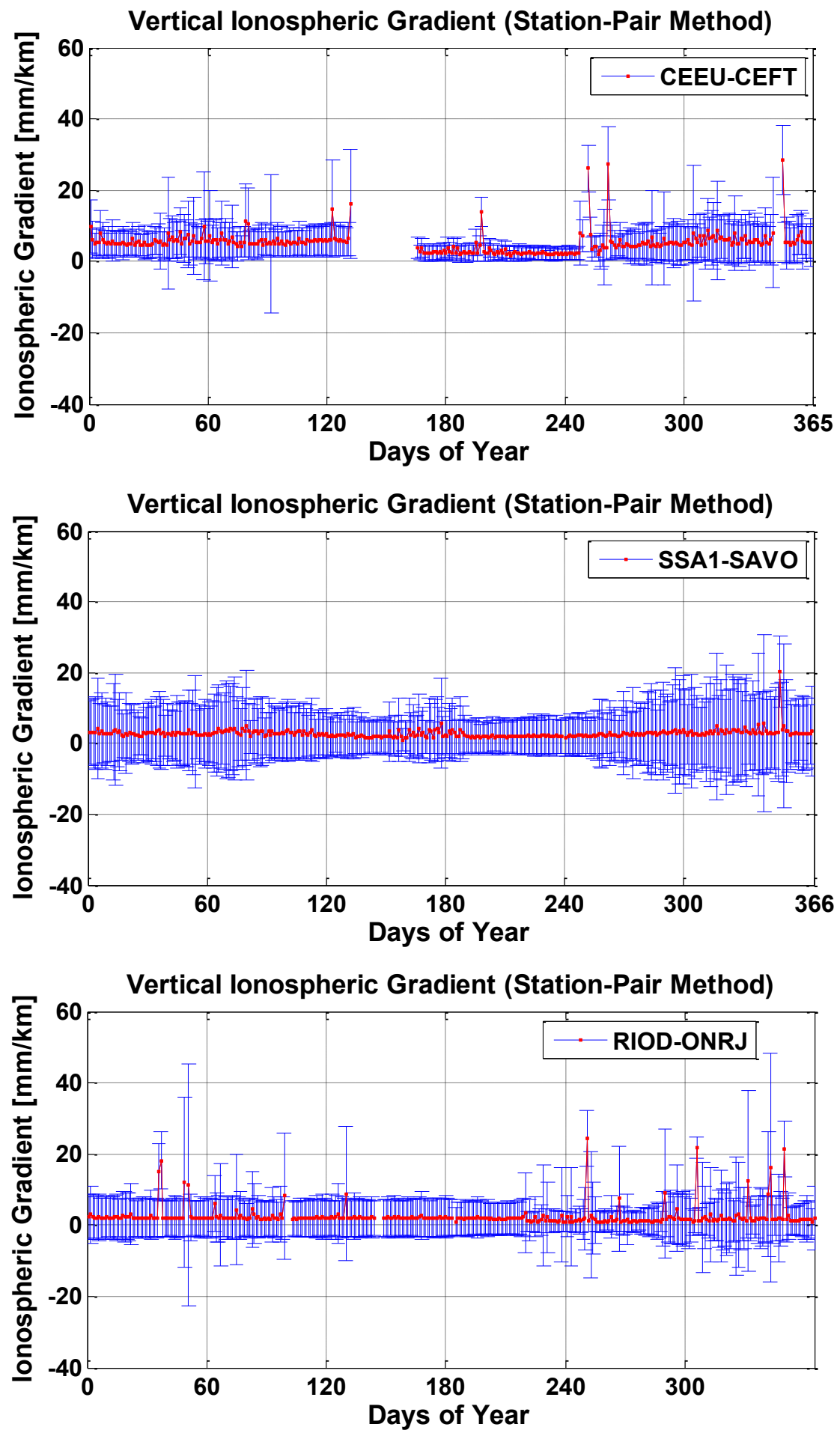

Figure 4.14: Daily values of the mean and standard deviation of VIG estimated by the station-pair method for CEEU and CEFT stations (top); SSA1 and SAVO stations (middle); RIOD and ONRJ stations (bottom) in 2013.

The estimation of the $\sigma_{\text {vert_iono_grad }}$ is important to evaluate the protection levels that will be presented in Chapter 7. 


\subsection{Time Variation of the Daily Maximum Vertical lonospheric Delay Gradient Using the Station-Pair Method}

This section presents the time variation of the daily maximumVIG using the station-pair method for the ONRJ-RIOD, SSA1-SAVO and CEEU-CEFT, station pairs. The description of the station pairs is contained in Table 4.2. Figures 4.15 and 4.16 show the results for the years 2008 (solar-minimum) and 2013 (solarmaximum), respectively.

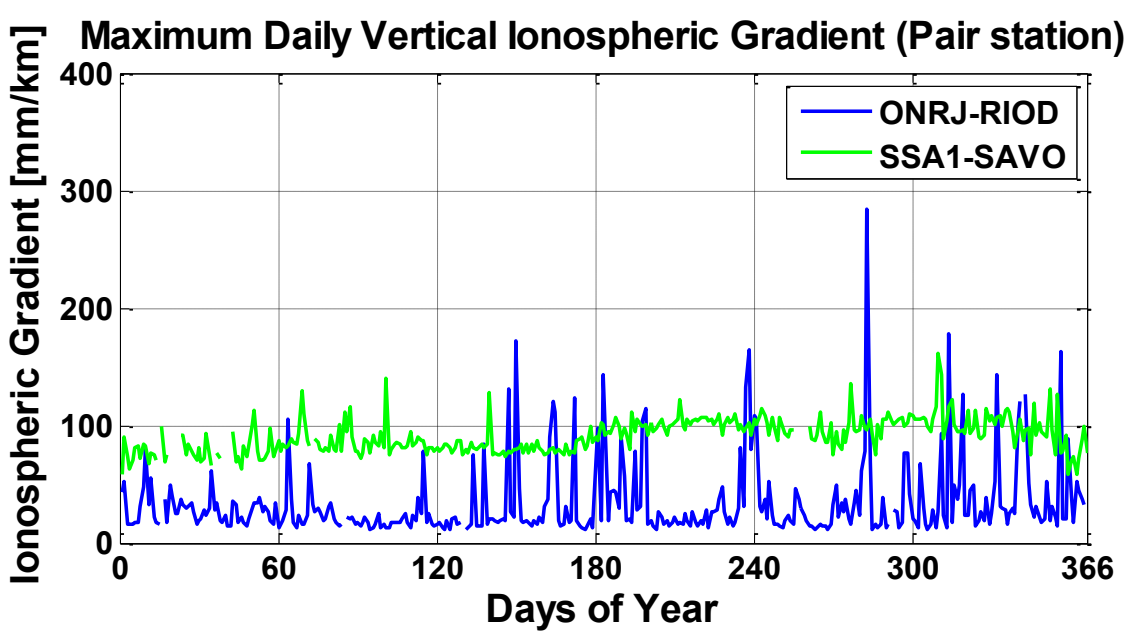

Figure 4.15: Daily maximum VIG estimated by the station-pair method based on data from the combinations of stations: ONRJ-RIOD (blue) and SSA1-SAVO (green) in 2008.

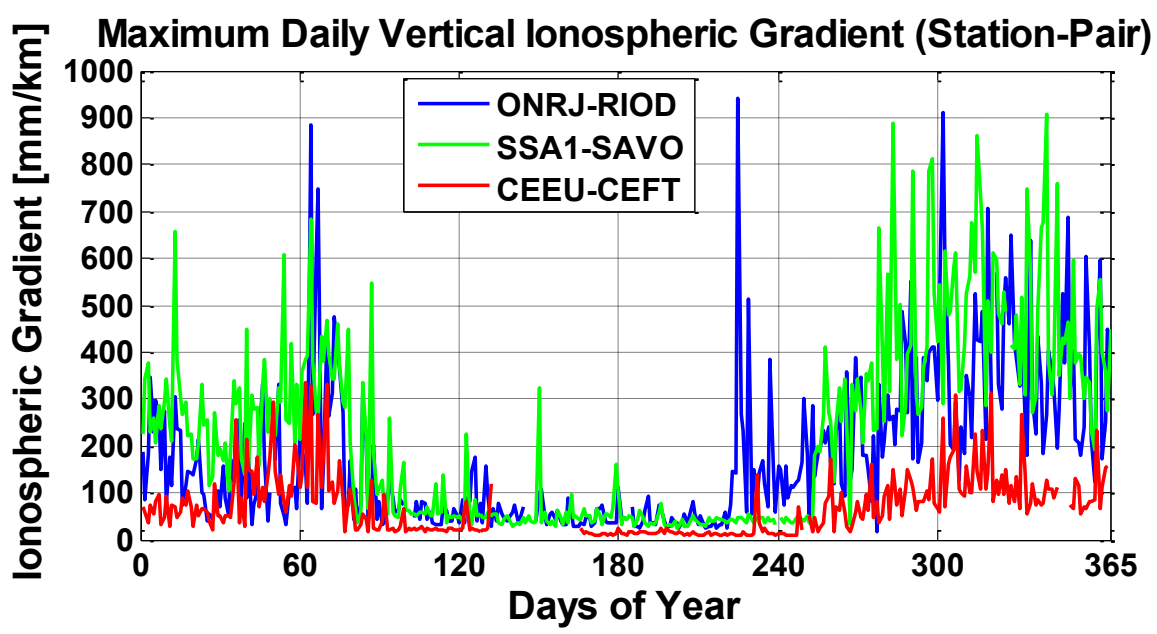

Figure 4.16: Daily maximum VIG estimated by the station-pair method based on data from the combinations of stations: ONRJ-RIOD (blue), SSA1-SAVO (green) and CEEU-CEFT (red), in 2013. 
Figure 4.15 shows that the pair: SSA1- SAVO presents large values of maximum vertical gradients in a large number of days in comparison to ONRJRIOD pairs corresponding to the year 2008, but there are some peak values corresponding to the RIOD station. The maximum gradients are equal to 284.32 $\mathrm{mm} / \mathrm{km}$ (ONRJ-RIOD), and $140.10 \mathrm{~mm} / \mathrm{km}$ (SSA1-SAVO).

Figure 4.16 shows that the smallest values of daily maximum VIG occur between the days 100 and 220 (April to August, covering the winter months of the southern hemisphere), as expected. In addition, SSA1- SAVO and ONRJ-RIOD pairs present large values of maximum vertical gradients in a large number of days between January to March and September to December corresponding to the year 2013. The results presented for ONRJ and RIOD are consistent with the results estimated by Pereira (2018) where the gradients are estimated for specific days in different seasons. On the other hand, CEEU-CEFT pair presents low gradients most part of the time. The maximum gradients are equal to $939.90 \mathrm{~mm} / \mathrm{km}$ (ONRJRIOD), $908.33 \mathrm{~mm} / \mathrm{km}$ (SSA1-SAVO) and $334.40 \mathrm{~mm} / \mathrm{km}$ (CEEU-CEFT).

The CCDFs of the vertical gradients estimated by the time-step method based on measurements from RIOD station and the station-pair method based on measurements from ONRJ and RIOD stations in 2013 are shown in Figure 4.17.

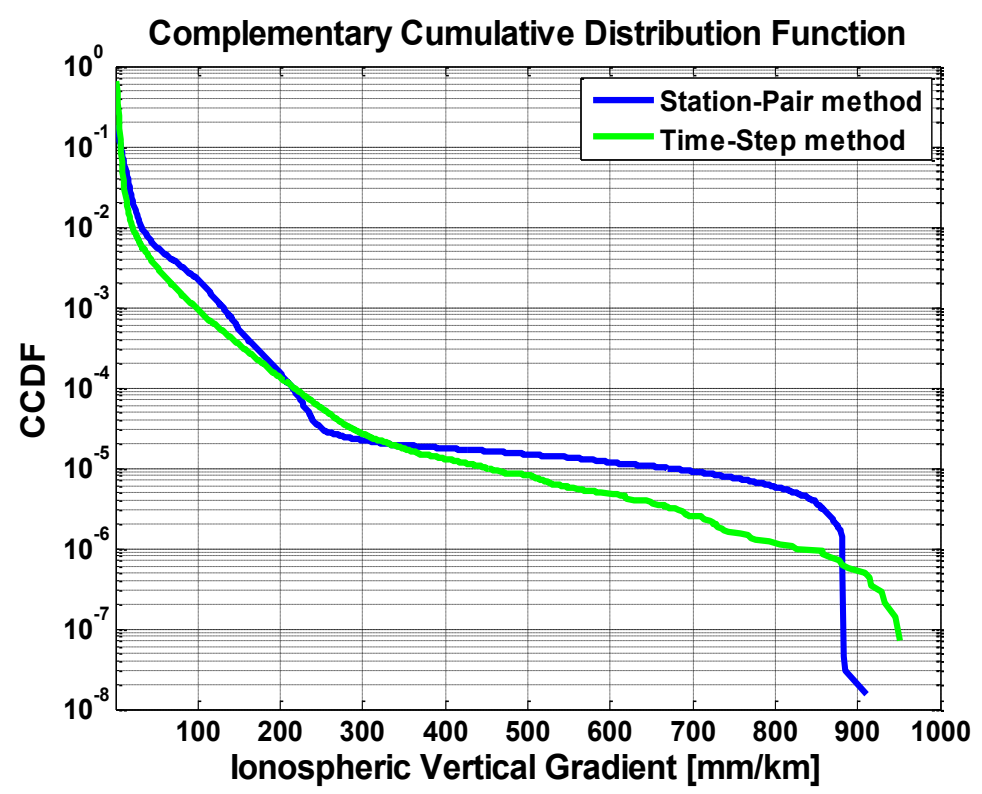

Figure 4.17: CCDFs of the vertical ionospheric delay gradients estimated by the time-step method (green) and station-pair method (blue) based on data from RIOD station and ONRJ-RIOD pair in 2013, respectively. 
For RIOD station, the time step method present high probability of presence of large ionospheric gradients. In addition, fixing the value of gradient (for example, $400 \mathrm{~mm} / \mathrm{km}$ ), the complementary cumulative distribution (Figure 4.17) shows that the probability that this value is exceeded is $1.31 \times 10^{-5}$ for the time-step method and $1.78 \times 10^{-5}$ for the station-pair method. In summary, the probability of RIOD station presents large gradients is small. 


\section{Signal in Space}

Chapter 2 presented a brief introduction to the GBAS Ground Facility (GGF) model, that apply a comprehensive set of monitoring algorithms to detect a varied array of possible failures in the GPS Signal in Space. This chapter introduces one the blocks of Figure 2.6 that is not part of the GGF model: the GPS Signal in Space (SIS) block. The transionospheric propagation of radio waves and the degradation of received GPS signals due to ionospheric effects are important sources of impairment of GBAS operation, being the focus of this Thesis. For this reason, the emphasis in this chapter will be on how to generate GPS observables and how to inject them into the references antennas of the GBAS and aircraft receivers. For completeness, non ionospheric effects will also be considered.

\subsection{Pseudorange, Carrier Phase and Received Signal Power Models}

For each available channel between satellite $i$ and aircraft or reference station $j$ at each epoch $k$ (receiver clock reading at the time when the signal reached the receiver), the following expressions apply to the pseudorange $P R_{(i, j)}(k)$, carrier phase $\phi_{(i, j)}(k)$, and received power $C_{(i, j)}(k)$ of the GPS L1 signals (Seeber, 2003; Hoffman-Wellenhof et al., 2008; Monico, 2008)

$$
\begin{gathered}
P R_{(i, j)}(k)=\rho_{(i, j)}(k)+c \cdot\left[\Delta t_{r(j)}(k)-\Delta t_{s(i)}(k)\right]+I_{(i, j)}(k)+T_{(i, j)}(k) \\
+m_{P R(i, j)}(k)+v_{P R(i, j)}(k) \\
\begin{aligned}
\phi_{(i, j)}(k)=\rho_{(i, j)}(k)+c \cdot\left[\Delta t_{r(j)}(k)-\Delta t_{s(i)}(k)\right]-I_{(i, j)}(k)+T_{(i, j)}(k) \\
+m_{\phi(i, j)}(k)+\lambda_{L 1} N_{(i, j)}(k)+\phi_{I s \operatorname{sint}(i, j)}(k)+v_{\phi(i, j)}(k)
\end{aligned} \\
C_{(i, j)}(k)=E I R P_{(i, j)}(k)+L_{(i, j)}(k)+G_{R(i, j)}(k)+m_{C(i, j)}(k) \\
+20 \log \left[a_{I s c i n t(i, j)}(k)\right]+v_{C(i, j)}(k)
\end{gathered}
$$


where $\rho_{(i, j)}(k)$ is the geometric range; $c$ is the speed of light in vacuum; $\Delta t_{r(j)}(k)$ and $\Delta t_{s(i)}(k)$ are the receiver and satellite clock errors; $I_{(i, j)}(k)$ and $T_{(i, j)}(k)$ represent the ionospheric and tropospheric delays; $m_{\mathrm{PR}(i, j)}(k), m_{\phi(i, j)}(k)$, and $m_{\mathrm{C}(i, j)}(k)$ are associated with multipath effects on pseudorange, carrier phase, and amplitude; $v_{\mathrm{PR}(i, j)}(k), v_{\phi(i, j)}(k)$, and $v_{C(i, j)}(k)$ represent pseudorange, carrier phase, and received power random errors; $\lambda_{L 1}=0.1902 \mathrm{~m}$ is the wavelength; $N_{(i, j)}(k)$ is an integer number representing the cycle ambiguity; $\phi_{\operatorname{Iscint}(i, j)}(k)$ and $a_{I s c i n t(i, j)}(k)$ represent phase and amplitude ionospheric scintillation effects; $\operatorname{EIRP}_{(i, j)}(k)$ is the effective isotropic radiated power of each satellite transmitter in the receiver $j$ direction; $G_{R(i, j)}(k)$ is the gain of the receiver antenna $j$ in the satellite $i$ direction; and $L_{(i, j)}(k)$ is the free-space path loss, represented by

$$
L_{(i, j)}(k)=-27.55+20 \log \left(f_{L 1, M H z}\right)+20 \log \left[\rho_{(i, j)}(k)\right]
$$

where $f_{L 1, M h z}=1575.42 \mathrm{MHz}$ is the frequency of L1 carrier and $\rho_{(i, j)}(k)$ is the geometric range between satellite and receiver. All terms in equations (5.1) and (5.2) are expressed in meters. The first two terms in equation (5.3) are expressed in $d B W$, while the other terms are dimensionless. Note the multiplicative and additive effects of the amplitude and phase scintillation terms in $C_{(i, j)}(k)$ and $\phi_{(i, j)}(k)$, respectively.

\subsubsection{Geometric Range $\rho_{(i, j)}(k)$}

The geometric range is the Euclidean distance between the satellite and the aircraft or reference station positions at the transmission and reception time, respectively, and can be expressed as

$$
\rho_{(i, j)}(k)=\sqrt{\left(x_{i}(k)-x_{j}(k)\right)^{2}+\left(y_{i}(k)-y_{j}(k)\right)^{2}+\left(z_{i}(k)-z_{j}(k)\right)^{2}}
$$

The terms $\left(x_{j}(k), y_{j}(k), z_{j}(k)\right)$ describe the aircraft or reference station $j$ coordinates in Earth-centered, Earth-fixed (ECEF) reference frame at reception time $k$. The positions of the reference stations are known and do not vary along the 
epochs. To simplify notation, the terms $\left(x_{i}(k), y_{i}(k), z_{i}(k)\right)$ represent the satellite $i$ coordinates in the same reference frame at the transmission time associated with the reception time $k$.

The algorithm used to estimate the satellite coordinates using the Keplerian parameters from Navigation RINEX files and the transmission time are described in Appendix A.

\subsubsection{Satellite Clock Errors $\Delta t_{S(i)}(k)$}

The satellite clock error can be estimated using the following equation (Seeber, 2003)

$$
\Delta t_{S(i)}(k)=a_{0}+a_{1}\left(t^{s}-t_{o e}\right)+a_{2}\left(t^{s}-t_{o e}\right)^{2}+\Delta t_{r e l}
$$

where $a_{0}, a_{1}$ and $a_{2}$ are the coefficients of satellite clock offset, $t^{s}$ is the transmission time of the satellite signal, $t_{o e}$ is the reference time for the coefficients expressed in GPS time and $\Delta t_{r e l}$ is the relativistic clock correction. Most terms of equation (5.6) can be retrieved from navigation RINEX files. The only exception is the transmission time $t^{s}$ of the satellite signal, estimated using a recursive method based on the geometric range and described in Appendix A.

A correction for relativistic effects $\Delta t_{r e l}$ is required because the satellite clock and the main receiver clock that defines the GPS system time operate at places with different gravitational potential and move with different velocities. The following expression denotes this correction (Kaplan, 1996)

$$
\Delta t_{r e l}=F e \sqrt{a} \sin E_{k}=\left(\frac{-2 \sqrt{G M}}{c^{2}}\right) e \sqrt{a} \sin E_{k}
$$

where $G M$ represents the gravitational constant, $e$ is the satellite orbital eccentricity, $a$ represents the semimajor axis of the satellite orbit, and $E_{k}$ is the eccentric anomaly of the satellite orbit at the transmission time $t^{S}$. 


\subsection{3. lonospheric Delay $I_{(i, j)}(k)$}

The ionospheric delay for each channel is represented by

$$
I_{(i, j)}(k)=\frac{40.3}{f_{L 1}^{2}} \operatorname{sTEC}_{(i, j)}(k)=\frac{40.3}{f_{L 1}^{2}} \frac{\left(v T E C_{I P P}^{I R I}+v \operatorname{TEC}_{I P P}^{r e s}\right)_{(i, j)}(k)}{S\left[\varepsilon_{(i, j)}(k)\right]}
$$

where $S\left[\varepsilon_{(i, j)}(k)\right]$ is the slant factor

$$
S\left[\varepsilon_{(i, j)}(k)\right]=\frac{1}{\cos \left[\arcsin \left(\frac{R_{E} \cos \varepsilon_{(i, j)}(k)}{R_{E}+H}\right)\right]}
$$

In expression $(5.9), \varepsilon_{(i, j)}(k)$ is the elevation angle for each channel and epoch, $R_{E}$ is the Earth radius, and $H$ is the height of the ionospheric layer, assumed to be 400 $\mathrm{km}$.

From Section 3.6.6, the residual term $v T E C_{I P P(i, j)}^{r e s}(k)$ is expressed as

$$
v T E C_{I P P(i, j)}^{r e s}(k)=v T E C_{R B M C(i, j)}(k)-v T E C_{I R I(i, j)}(k)
$$

where $v T E C_{R B M C(i, j)}(k)$ is the vertical TEC estimated from original RINEX RBMC network files using the methodology presented in Chapter 3 and $v T E C_{I R I(i, j)}(k)$ is the vertical TEC estimated from the IRI 2016 model at each corresponding epoch and ionospheric pierce point.

For each channel satellite - reference station or satellite - aircraft pair and each epoch, the random variable $v T E C_{I P P}^{\text {res }}$ is sorted from the collection of cumulative distribution functions estimated from the 72 histograms displayed in Figure 3.16, classified according to five geophysical parameters (geomagnetic latitude, local time, EPB and scintillation seasons in the Brazilian sector, solar activity, and geomagnetic activity) described in Section 3.6.5. 


\subsubsection{Tropospheric Delay $T_{(i, j)}(k)$}

Due to the refractive index of the Earth's neutral atmosphere, GNSS microwave signals experience tropospheric propagation delays. The total tropospheric delay along a particular satellite - receiver slant path can be divided into a hydrostatic and a wet component (Seeber, 2003; Hoffman-Wellenhof et al., 2008; Monico, 2008). Using mapping functions, these two delay components are projected into the zenith direction and vice versa. The present formulation adopted the Saastamoinen delay model (Saastamoinen, 1972), specified by

$$
T_{(i, j)}(k)=T_{Z H D} \cdot m h\left(\varepsilon_{(i, j)}(k)\right)+T_{Z W D} \cdot m w\left(\varepsilon_{(i, j)}(k)\right)
$$

where $T_{Z H D}$ and $T_{Z W D}$ represent the zenith hydrostatic and wet delays, respectively, and $m h\left(\varepsilon_{(i, j)}(k)\right)$ and $m w\left(\varepsilon_{(i, j)}(k)\right)$ are the Niell mapping functions (Niell, 1996).

The zenith hydrostatic delay $T_{Z H D}$ can be modeled using the total pressure at the receiving antenna site, using the following equation

$$
T_{Z H D}=\frac{0.0022767 \cdot p}{1-0.00266 \cdot \cos (2 \varphi)-\left(2.8 \cdot 10^{-7}\right) \cdot h}
$$

where $p[\mathrm{hPa}]$ is total pressure at the receiving antenna site in, $\varphi$ is the ellipsoid latitude, and $h[\mathrm{~m}]$ is the surface height above the ellipsoid.

The zenith wet delay $T_{Z W D}$ can be modeled using the pressure of water vapor at the receiving antenna site, using the following expression

$$
\begin{aligned}
& T_{Z W D}=\frac{0.0022767}{1-0.00266 \cdot \cos (2 \varphi)-\left(2.8 \cdot 10^{-7}\right) \cdot h} \\
& \cdot\left[\left(\frac{1255}{t+273.15}+0.05\right) e\right]
\end{aligned}
$$


where $t\left[{ }^{\circ} \mathrm{C}\right]$ is the surface temperature, $e[\mathrm{hPa}]$ is the surface partial pressure of water vapor, which can be expressed as a function of humidity $H$ and pressure of saturation water vapor $e_{s}$ (Recommendation ITU-R P.453-13, 2017)

$$
\begin{gathered}
e=\frac{H \cdot e_{s}}{100} \\
e_{s}=F \cdot 6.1121 \cdot \exp \left[\frac{\left(18.678-\frac{t}{234.84}\right) \cdot t}{t+257.14}\right] \\
F=1+10^{-4}\left[7.2+p \cdot\left(0.0320+5.9 \cdot 10^{-6} \cdot t^{2}\right)\right] .
\end{gathered}
$$

\section{Niell mapping function}

The Niell hydrostatic mapping function $\operatorname{mh}\left(\varepsilon_{(i, j)}(k)\right)$, which provides relatively small errors (Schüler, 2001), can be expressed as

$$
m h\left(\varepsilon_{(i, j)}(k)\right)=\frac{1+\frac{a}{1+\frac{b}{1+c}}}{\sin \left(\varepsilon_{(i, j)}(k)\right)+\frac{a}{\sin \left(\varepsilon_{(i, j)}(k)\right)+\frac{b}{\sin \left(\varepsilon_{(i, j)}(k)\right)+c}}}+\Delta m
$$

where $a, b$, and $c$ are the mapping functions coefficients, expressed as

$$
\left(\begin{array}{l}
a \\
b \\
c
\end{array}\right)(\varphi, D o Y)=\left(\begin{array}{l}
a \\
b \\
c
\end{array}\right)_{a v g}(\varphi)-\left(\begin{array}{l}
a \\
b \\
c
\end{array}\right)_{a m p l}(\varphi) \cos \left(2 \pi \frac{D o Y-D o Y_{0}}{365.25}\right)
$$

The mapping function coefficient are separated into average and amplitude, $\varphi$ is the ellipsoid latitude, $D o Y$ is the day of the year and $D o Y_{0}$ is the day of year for maximum winter. $D o Y_{0}$ is set to 28 or 211 for the northern or southern hemispheres, respectively. The average and amplitude coefficients are provided by Tables 5.1 and 5.2 for particular latitudes. Intermediate values should be obtained by the application of linear interpolation to the Table values. 
Table 5.1: Coefficients of the Niell hydrostatic mapping function (average values). [Reproduced from Niell, 1996]

\begin{tabular}{|c|c|c|c|}
\hline $\begin{array}{c}\text { Coefficient } \\
\text { Latitude }\end{array}$ & $\mathbf{a}_{\text {avg }}$ & $\mathbf{b}_{\text {avg }}$ & $\mathbf{c}_{\text {avg }}$ \\
\hline$\varphi=15^{\circ}$ & $1.2769934 \cdot 10^{-3}$ & $2.9153695 \cdot 10^{-3}$ & $62.610505 \cdot 10^{-3}$ \\
\hline$\varphi=30^{\circ}$ & $1.2683230 \cdot 10^{-3}$ & $2.9152299 \cdot 10^{-3}$ & $62.837393 \cdot 10^{-3}$ \\
\hline$\varphi=45^{\circ}$ & $1.2465397 \cdot 10^{-3}$ & $2.9288445 \cdot 10^{-3}$ & $63.721774 \cdot 10^{-3}$ \\
\hline$\varphi=60^{\circ}$ & $1.2196049 \cdot 10^{-3}$ & $2.9022565 \cdot 10^{-3}$ & $63.824265 \cdot 10^{-3}$ \\
\hline$\varphi=75^{\circ}$ & $1.2045996 \cdot 10^{-3}$ & $2.9024912 \cdot 10^{-3}$ & $64.258455 \cdot 10^{-3}$ \\
\hline
\end{tabular}

Table 5.2: Amplitude coefficients of the Niell hydrostatic mapping function.

[Reproduced from Niell, 1996]

\begin{tabular}{|c|c|c|c|}
\hline $\begin{array}{c}\text { Coefficient } \\
\text { Latitude }\end{array}$ & $\mathbf{a}_{\text {ampl }}$ & $\mathbf{b}_{\text {ampl }}$ & $\mathbf{c}_{\text {ampl }}$ \\
\hline$\varphi=15^{\circ}$ & 0.0 & 0.0 & 0.0 \\
\hline$\varphi=30^{\circ}$ & $1.2709626 \cdot 10^{-5}$ & $2.1414979 \cdot 10^{-5}$ & $9.0128400 \cdot 10^{-5}$ \\
\hline$\varphi=45^{\circ}$ & $2.6523662 \cdot 10^{-5}$ & $3.0160779 \cdot 10^{-5}$ & $4.3497037 \cdot 10^{-5}$ \\
\hline$\varphi=60^{\circ}$ & $3.4000452 \cdot 10^{-5}$ & $7.2562722 \cdot 10^{-5}$ & $84.795348 \cdot 10^{-5}$ \\
\hline$\varphi=75^{\circ}$ & $4.1202191 \cdot 10^{-5}$ & $11.723375 \cdot 10^{-5}$ & $170.37206 \cdot 10^{-5}$ \\
\hline
\end{tabular}

The height correction of the Niell hydrostatic mapping function $\Delta m$ can be estimated using the following equation

$$
\begin{gathered}
\Delta m=\left[\frac{1}{\sin \left(\varepsilon_{(i, j)}(k)\right)}-m_{h t}\left(\varepsilon_{(i, j)}(k)\right)\right] \cdot h \\
m_{h t}\left(\varepsilon_{(i, j)}(k)\right)=\frac{1+\frac{a_{h t}}{1+\frac{b_{h t}}{1+c_{h t}}}}{\sin \left(\varepsilon_{(i, j)}(k)\right)+\frac{a_{h t}}{\sin \left(\varepsilon_{(i, j)}(k)\right)+\frac{b_{h t}}{\sin \left(\varepsilon_{(i, j)}(k)\right)+c_{h t}}}}
\end{gathered}
$$


where $a_{h t}, b_{h t}, c_{h t}$ are the coefficients of the Niell hydrostatic mapping function shown in Table 5.3, and $\varepsilon_{(i, j)}(k)$ is the elevation angle.

Table 5.3: Coefficients of the Niell hydrostatic mapping function (height correction). [Reproduced from Niell, 1996]

\begin{tabular}{|c|c|}
\hline Coefficient & Height Correction \\
\hline$a_{h t}$ & $2.53 \cdot 10^{-5}$ \\
\hline$b_{h t}$ & $5.49 \cdot 10^{-3}$ \\
\hline$c_{h t}$ & $1.14 \cdot 10^{-3}$ \\
\hline
\end{tabular}

The Niell wet mapping function $m w\left(\varepsilon_{(i, j)}(k)\right)$ can be similarly expressed as

$$
m w\left(\varepsilon_{(i, j)}(k)\right)=\frac{1+\frac{a_{w}}{1+\frac{b_{w}}{1+c_{w}}}}{\sin \left(\varepsilon_{(i, j)}(k)\right)+\frac{a_{w}}{\sin \left(\varepsilon_{(i, j)}(k)\right)+\frac{b_{w}}{\sin \left(\varepsilon_{(i, j)}(k)\right)+c_{w}}}}
$$

The coefficients for wet mapping function are provided by Table 5.4 for particular latitudes. Intermediate values should again be obtained by the application of linear interpolation to the Table values. Seasonal variations are not modeled for the wet component.

Table 5.4: Coefficients of the Niell wet mapping function.

[Reproduced from Niell, 1996]

\begin{tabular}{|c|c|c|c|}
\hline $\begin{array}{c}\text { Coefficient } \\
\text { Latitude }\end{array}$ & $\mathbf{a}_{\mathbf{w}}$ & $\mathbf{b}_{\mathbf{w}}$ & $\mathbf{c}_{\mathbf{w}}$ \\
\hline$\varphi=15^{\circ}$ & $5.8021897 \cdot 10^{-4}$ & $1.4275268 \cdot 10^{-3}$ & $4.3472961 \cdot 10^{-2}$ \\
\hline$\varphi=30^{\circ}$ & $5.6794867 \cdot 10^{-4}$ & $1.5138625 \cdot 10^{-3}$ & $4.6729510 \cdot 10^{-2}$ \\
\hline$\varphi=45^{\circ}$ & $5.8118019 \cdot 10^{-4}$ & $1.4572752 \cdot 10^{-3}$ & $4.3908931 \cdot 10^{-2}$ \\
\hline$\varphi=60^{\circ}$ & $5.9725542 \cdot 10^{-4}$ & $1.5007428 \cdot 10^{-3}$ & $4.4626982 \cdot 10^{-2}$ \\
\hline$\varphi=75^{\circ}$ & $6.1641693 \cdot 10^{-4}$ & $1.7599082 \cdot 10^{-3}$ & $5.4736038 \cdot 10^{-2}$ \\
\hline
\end{tabular}


The estimation of the tropospheric delay as a function of elevation with basis on the model described in the present Subsection for a receiver station in Rio de Janeiro using the parameters DoY $=256$, humidity $=80 \%$, temperature $\mathrm{t}=25^{\circ} \mathrm{C}$, atmospheric pressure $\mathrm{p}=1012 \mathrm{hPa}$ is displayed in Figure 5.1. It is observed that, as the elevation increases from $30^{\circ}$, the tropospheric delay decreases from approximately $5.2 \mathrm{~m}$ to $2.5 \mathrm{~m}$.

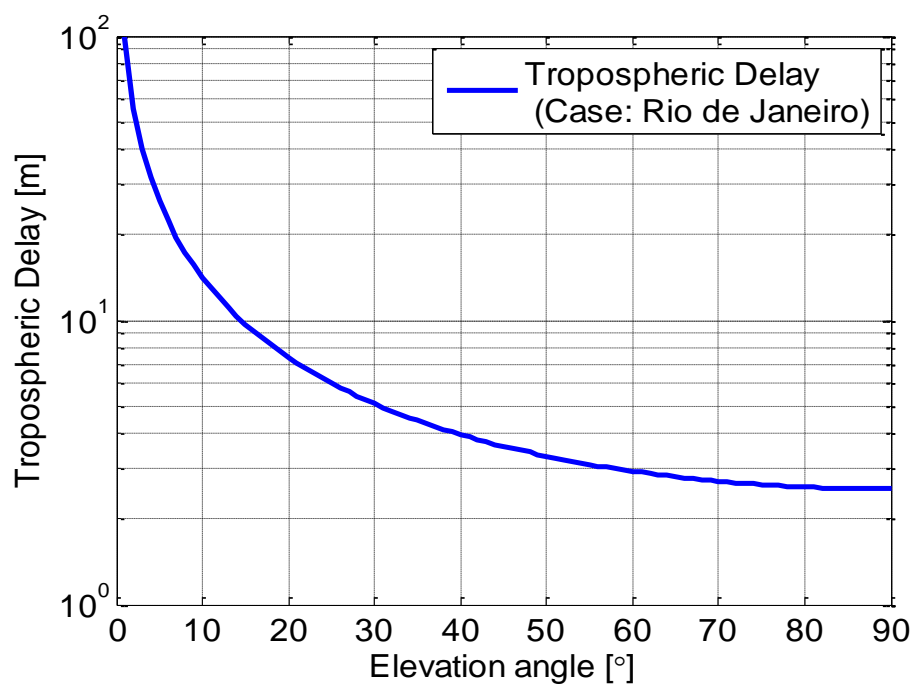

Figure 5.1: Tropospheric delay as a function of elevation angle estimated for Rio de Janeiro receiver station $\left(\mathrm{DoY}=256\right.$, humidity $=80 \%$, temperature $\mathrm{t}=25^{\circ} \mathrm{C}$, atmospheric pressure $=1012 \mathrm{hPa}$ ).

\subsubsection{Multipath}

Multipath occurs when the signal energy propagates between the transmitter and receiver along different paths. For example, the transmitted signals from GNSS satellite reach the GPS receiving antenna along the direct path, as well as after one or more reflections from the ground, a building or another surface. These reflected signals can reach the receiving antenna with different delays. Their combination can produce constructive or destructive interference (that is, signal enhancement or fading, respectively), depending on the delays. Multipath affects the pseudorange, phase and received power measurements. This work will assume the well-known two-ray model. That is, a radio propagation model that considers the direct ray and a single reflected path, assuming line-of-sight (LOS) conditions between the satellite and receiving antennas (Jakes, 1974), as illustrated in Figure 5.2. The 
ground surface is characterized by a reflection coefficient that depends on its material properties and the angle of incidence, as well as the wave polarization.

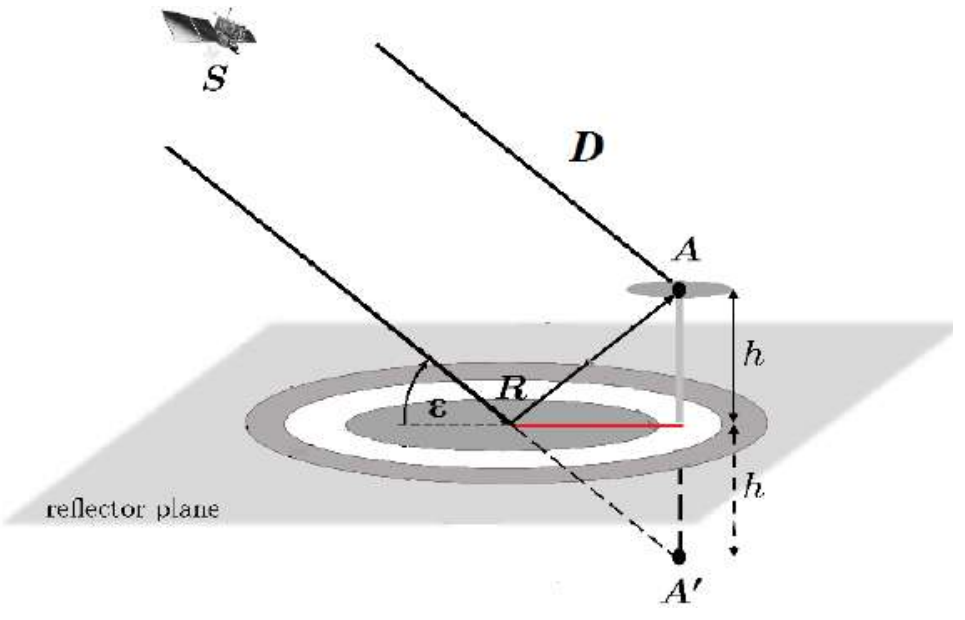

Figure 5.2: Two-ray ground reflection model.

The electric field at the receiving antenna, considering its amplitude radiation pattern, is the sum of the direct and reflected components

$$
\vec{E}_{m}=V_{o} g\left(\theta_{1}\right)(1, i, 0) \frac{e^{i k R_{1}}}{R_{1}}+V_{o} g\left(\theta_{2}\right)\left(R_{V}, i R_{H}, 0\right) \frac{e^{i k R_{2}}}{R_{2}}
$$

where $i=\sqrt{-1} ; k=\omega / c=2 \pi / \lambda$ is the wavenumber; $\omega=2 \pi f$ is the angular frequency; a harmonic time dependence according to $\exp (-i \omega t)$ is implicit; $R_{1}$ and $R_{2}$ are the distances of the direct and reflected paths between the GPS satellite and the receiver, respectively; $g\left(\theta_{1}\right)$ and $g\left(\theta_{2}\right)$ are the values of the amplitude pattern of the receiving antenna for the direct and reflected paths between the GPS satellite and the receiver, respectively. The vector $(1, i, 0)$ associates the vertical and horizontal components with local $x$ - and $y$-axes, characterizing a right-hand circularly polarized (RHCP) field along the direct path, for a wave propagating away from an observer along the local $z$-axis. On the other hand, the vector $\left(R_{V}, i R_{H}, 0\right)$ results from the ground reflection of a similar wave, as shown in Figure 5.2. The parameters $R_{V}$ and $R_{H}$ are the reflection coefficients of linearly polarized waves in the vertical and horizontal planes, respectively.

It is easy to see that the second term in equation (5.22), representing the reflected wave, can be rearranged in the form 


$$
\vec{E}_{m r}=V_{o} g\left(\theta_{2}\right)\left[\frac{R_{V}+R_{H}}{2}(1, i, 0)+\frac{R_{V}-R_{H}}{2}(1,-i, 0)\right] \frac{e^{i k R_{2}}}{R_{2}}
$$

That is, the ground-reflected component can be expressed as a weighted sum of RHCP and LHCP waves (first and second terms in the previous equation, respectively).

It should be remarked that real antennas, even when designed to transmit or receive signals with a single polarization (RHCP, in the present case), will always transmit or receive relatively weak signals with the orthogonal polarization (LHCP, in the present case). Thus, all the depolarization procedures sketched in Figure 5.3 will be present in the transmission and reception of energy along the direct and reflected rays. In this Figure, the amplitudes of the components decrease according to the following representation: thick continuous, thin continuous, thick dashed, thin dashed and dotted lines. Thus, the first terms in equations (5.22) and (5.23), labeled $\mathrm{D}_{1}$ and $\mathrm{R}_{1}$ in Figure 5.3, represent the predominant components of the signal detected by the RHCP receiving antenna. The contributions of small RHCP terms (labeled $\mathrm{D}_{3}, \mathrm{R}_{3}, \mathrm{R}_{5}$, and $\mathrm{R}_{7}$ in Figure 5.3) will be incorporated into the random error terms of equations (5.1) to (5.3). Finally, LHCP terms of Figure 5.3 are not detected by the RHCP receiving antenna.

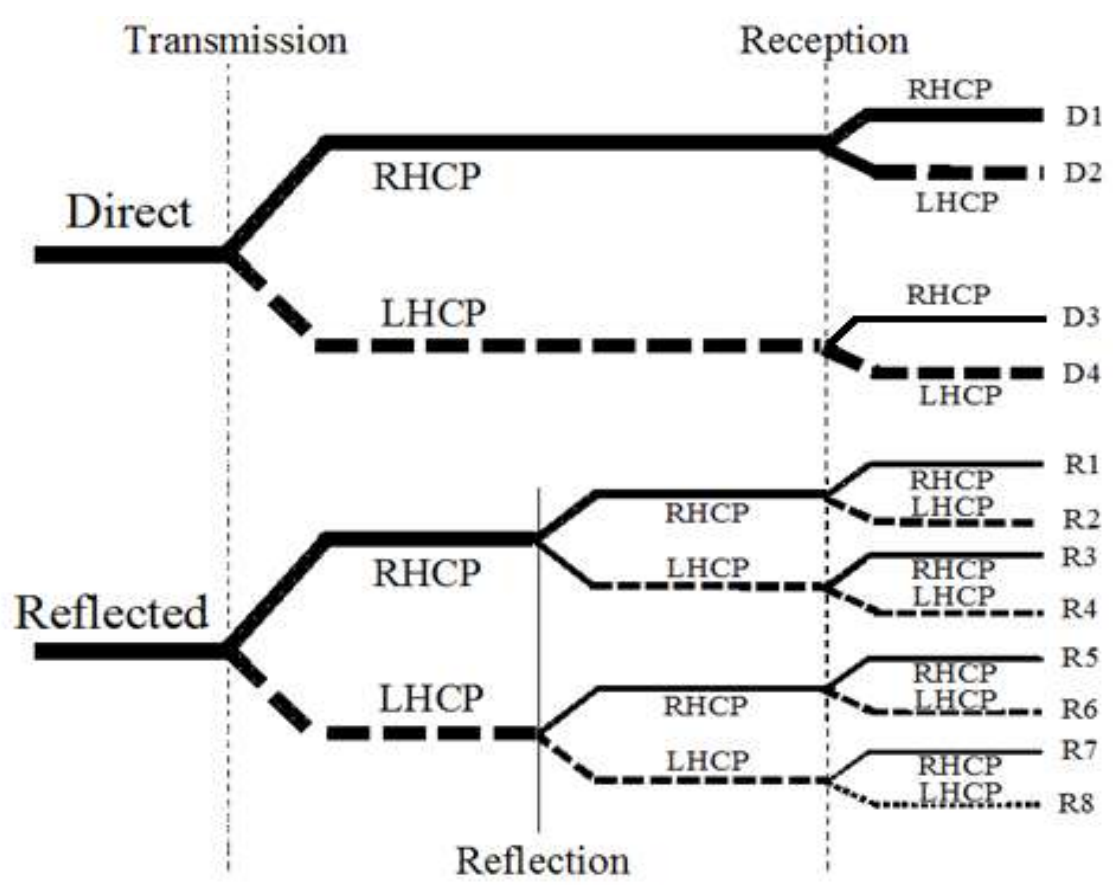

Figure 5.3: Depolarization of the signal due to multipath. 
From the above discussion, it is seen that the predominant components of the signal detected by the RHCP receiving antenna, represented by the first terms in equations (5.22) and (5.23), can be reorganized in the normalized form

$$
E_{m}=g\left(\theta_{1}\right) \frac{e^{i k R_{1}}}{R_{1}}\left\{1+\frac{g\left(\theta_{2}\right)}{g\left(\theta_{1}\right)} \frac{R_{1}}{R_{2}} R_{F}^{r} e^{i k\left(R_{2}-R_{1}\right)}\right\}
$$

and the following term from equation (5.24) can be defined as

$$
\gamma=\frac{g\left(\theta_{2}\right)}{g\left(\theta_{1}\right)} \frac{R_{1}}{R_{2}} \cdot R_{F}^{r}\left(\varepsilon_{(i, j)}, \epsilon_{g}, \sigma_{g}, \delta h, f\right)=|\gamma| \exp \left(i \varphi_{R F}\right)
$$

Additionally, the reflection coefficient $R_{F}^{r}$ of a GPS RHCP signal, considering a rough surface, can be represented by a combination of terms (Beckmann and Spizzichino, 1963; Miller et al., 1984)

$$
R_{F}^{r}=\frac{R_{V}+R_{H}}{2}=\frac{\Gamma_{v}+\Gamma_{h}}{2} \cdot C(x)=\frac{\Gamma_{v}+\Gamma_{h}}{2} \cdot e^{\left(-\frac{1}{2} g^{2}\right)} I_{0}\left(\frac{1}{2} g^{2}\right)
$$

The parameters $\Gamma_{h}$ and $\Gamma_{v}$ are the Fresnel reflection coefficients for horizontallyand vertically-polarized signals, which depend on the elevation angle and the electrical properties of the reflection surface, expressed as (Balanis, 1989)

$$
\begin{gathered}
\Gamma_{h}=\frac{\sin \varepsilon_{(i, j)}-\sqrt{\epsilon_{g}-\cos ^{2} \varepsilon_{(i, j)}}}{\sin \varepsilon_{(i, j)}+\sqrt{\epsilon_{g}-\cos ^{2} \varepsilon_{(i, j)}}} \\
\Gamma_{v}=\frac{\epsilon_{g} \cdot \sin \varepsilon_{(i, j)}-\sqrt{\epsilon_{g}-\cos ^{2} \varepsilon_{(i, j)}}}{\epsilon_{g} \cdot \sin \varepsilon_{(i, j)}+\sqrt{\epsilon_{g}-\cos ^{2} \varepsilon_{(i, j)}}}
\end{gathered}
$$

where $\epsilon_{g}$ is the complex relative permittivity of the ground given by

$$
\epsilon_{g}=\epsilon_{r}+i 60 \lambda \sigma_{g}
$$


where $\epsilon_{r}$ is the dielectric constant, and $\sigma_{g}$ is the ground conductivity [S/m]. In equation (5.26), $I_{0}(x)$ is the modified Bessel function of zero order and $g$ is the parameter used to define the Rayleigh roughness criterion

$$
g=4 \pi\left(\frac{\sigma_{h}}{\lambda}\right) \sin \left(\varepsilon_{(i, j)}(k)\right)
$$

where $\sigma_{h}$ is the standard deviation of the surface height fluctuations (Miller et al., 1984).

Figure 5.4 presents estimated values for the magnitude (blue line for horizontal polarization, red line for vertical polarization and green line for right hand circular polarization RHCP, respectively) and phase (blue dashed line for horizontal polarization, red dashed line for vertical polarization and green dashed for RHCP, respectively) of the Fresnel reflection coefficients using the electrical properties of wet ground $\left(\epsilon_{r}=30, \sigma_{g}=0.01 \mathrm{~S} / \mathrm{m}\right)$ and the L1 GPS frequency.

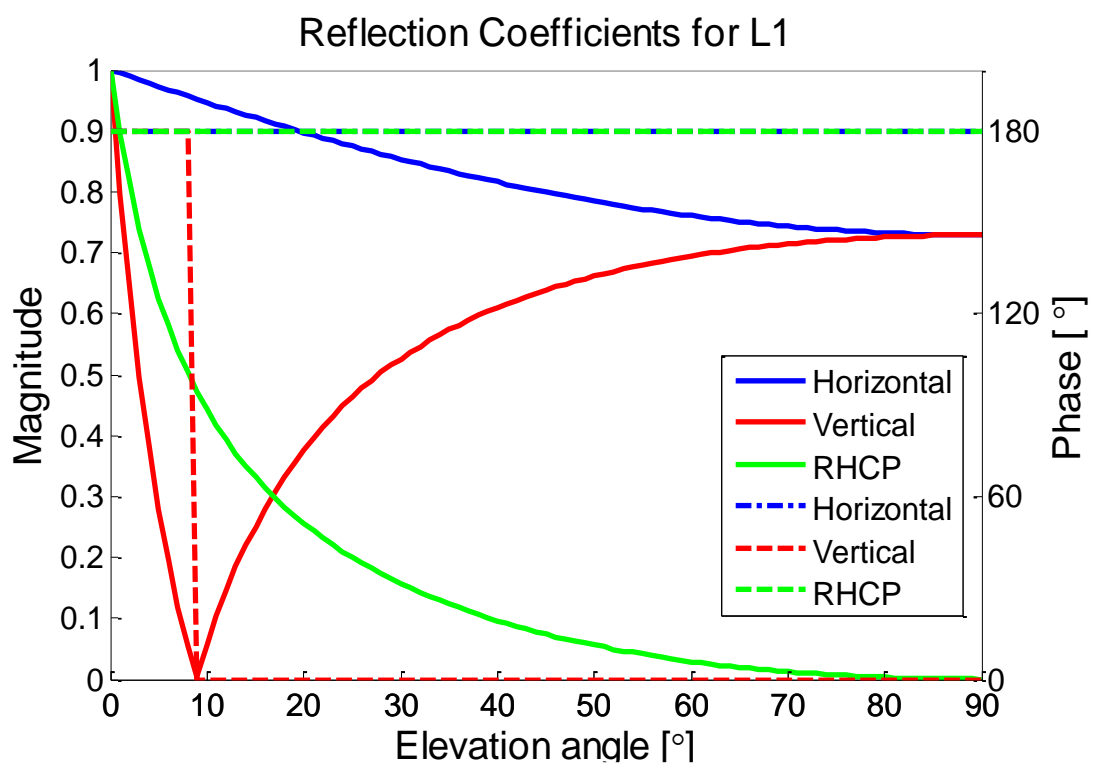

Figure 5.4: Magnitude (blue line for horizontal polarization and red line for vertical polarization, respectively) and phase (blue dashed line for horizontal polarization and red dashed line for vertical polarization, respectively) of the Fresnel reflection coefficients using the electrical properties of wet ground $\left(\epsilon_{r}=30, \sigma_{g}=0.01 \mathrm{~S} / \mathrm{m}\right)$. 
Substituting representation (5.25) into equation (5.24), with the help of the geometry in Figure 5.2, one gets

$$
E_{m}=g\left(\theta_{1}\right) \frac{e^{i k R_{1}}}{R_{1}}\left\{1+\gamma e^{i 2 k h \sin \left(\varepsilon_{(i, j)}(k)\right)}\right\}
$$

and rewriting this result to explicitly display the amplitude and phase of the term between curly brackets, it follows that

$$
\begin{aligned}
& E_{m}=g\left(\theta_{1}\right) \frac{e^{i k R_{1}}}{R_{1}} \sqrt{1+|\gamma|^{2}+2|\gamma| \cos \left(2 k h \sin \left(\varepsilon_{(i, j)}(k)\right)+\varphi_{R F}\right)} \cdot \\
& \cdot \exp \left\{i \cdot \arctan \left[\frac{|\gamma| \sin \left(2 k h \sin \left(\varepsilon_{(i, j)}(k)\right)+\varphi_{R F}\right)}{1+|\gamma| \cos \left(2 k h \sin \left(\varepsilon_{(i, j)}(k)\right)+\varphi_{R F}\right)}\right]\right\}
\end{aligned}
$$

With the help of expression (5.32), multipath effects on the carrier phase and received power can be modeled as follows. The mathematical derivation of the multipath induced pseudorange error by using a discriminator function can be found in works of (Byun et al., 2002; Ray et al., 2001).

\section{Carrier phase multipath error $\boldsymbol{m}_{\varphi(i, j)}(k)$}

The additive carrier phase multipath additive error is a fraction of the carrier wavelength and, from equation (5.32), can be represented by

$$
m_{\varphi(i, j)}(k)=\frac{\lambda}{2 \pi} \arctan \left[\frac{|\gamma| \sin \left(2 k h \sin \left(\varepsilon_{(i, j)}(k)+\varphi_{R F}\right)\right.}{1+|\gamma| \cos \left(2 k h \sin \left(\varepsilon_{(i, j)}(k)+\varphi_{R F}\right)\right.}\right]
$$

\section{Received power multipath error $\boldsymbol{m}_{C(i, j)}(k)$}

The multiplicative effect of multipath on the power of the received signal can be represented by

$$
m_{C(i, j)}(k)=10 \log \left[1+|\gamma|^{2}+2|\gamma| \cos \left(2 k h \sin \left(\varepsilon_{(i, j)}(k)+\varphi_{R F}\right)\right]\right.
$$




\section{Pseudorange multipath error $m_{P R(i, j)}(k)$}

Additionally, the effect of multipath on the pseudorange measurement can reach the length of a chip and can be represented in two cases depending the receiver correlation as (Byun et al., 2002)

Case 1 applies when $\tau<T-S+m_{P R(i, j)}(k)$

$$
m_{P R(i, j)}(k)=\frac{\tau|\gamma| \cos \left(2 k h \sin \left(\varepsilon_{(i, j)}(k)+\varphi_{R F}\right)\right.}{1+|\gamma| \cos \left(2 k h \sin \left(\varepsilon_{(i, j)}(k)+\varphi_{R F}\right)\right.}
$$

Case 2 applies when $T-S+m_{P R(i, j)}(k)<\tau<S+m_{P R(i, j)}(k)$

$$
m_{P R(i, j)}(k)=\frac{(T-S+\tau)|\gamma| \cos \left(2 k h \sin \left(\varepsilon_{(i, j)}(k)+\varphi_{R F}\right)\right.}{2+|\gamma| \cos \left(2 k h \sin \left(\varepsilon_{(i, j)}(k)+\varphi_{R F}\right)\right.}
$$

where $\mathrm{T}$ is the GPS C/A code chip length ( $\mathrm{T}=293 \mathrm{~m}), \mathrm{S}$ is sampling interval, both expressed in $[\mathrm{m}]$, and $\tau=2 h \cdot \sin \varepsilon_{(i, j)}(k)$.

\subsubsection{Amplitude $a_{I s c i n t(i, j)}(k)$ and Phase Scintillation $\phi_{I s c i n t(i, j)}(k)$}

The $S_{4}$ index indicates the severity of amplitude scintillation. It is defined as the standard deviation of the normalized received signal intensity (Briggs and Parkin, 1963; Crane, 1977; Basu, et al., 1999) and can be expressed as

$$
S_{4}=\sqrt{\frac{\left\langle I^{2}\right\rangle-\langle I\rangle^{2}}{\langle I\rangle^{2}}}
$$

where $I=|A|^{2}$ is the intensity, $A$ is the amplitude of the received signal, and \langle\rangle denotes an ensemble average. This single parameter has been used to specify cumulative distribution functions of amplitude scintillation. However, Alison et al. (2014) has shown that one-parameter cumulative distribution functions based on $S_{4}$ do not provide a flexible representation of amplitude scintillation, as illustrated in Figure 5.5. Indeed, Panels 5.5(a) and 5.5(b) show two different signals, indicated by the fade depths, with approximately the same value of $S_{4}$. Their differences are 
more evident in the associated occurrence histograms, represented by circles in Panel 5.5(c). Remember that $r^{2}$ represents values of the normalized received power.

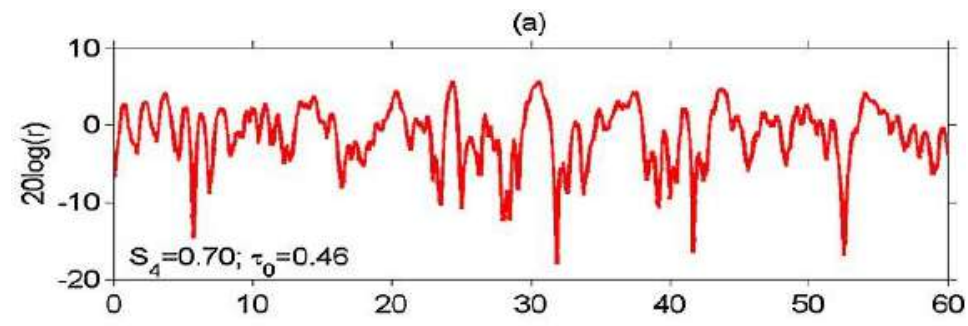

(b)

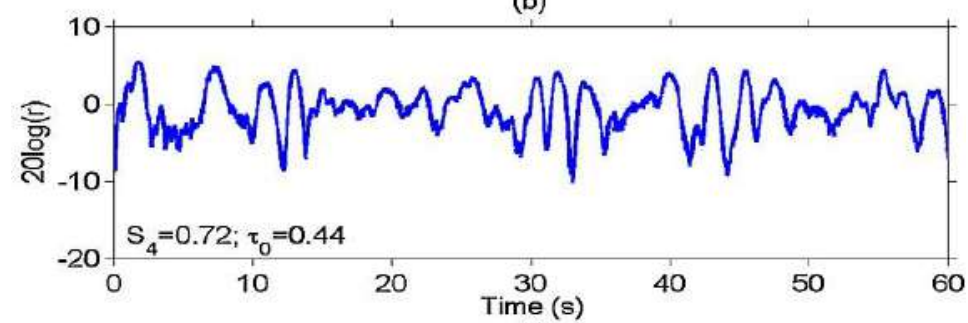

(c)

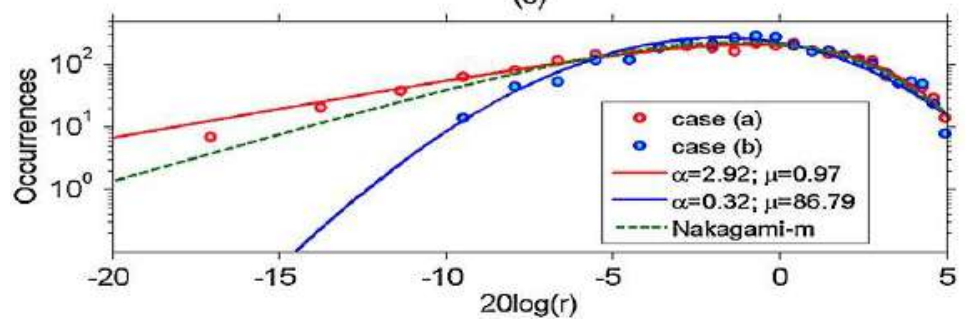

Figure 5.5: (a) and (b) Two signal records illustrating the differences between the occurrences of fade depths for signals with approximately the same scintillation index $S_{4}$ and decorrelation time $\tau_{o}$; (c) Corresponding measured occurrences and the best-fit $\alpha-\mu$ distributions (red and blue lines), as well as the Nakagami$m$ distribution with $m=1 / S_{4}^{2}=1 / 0.7^{2}=2.04$ (green line). [Reproduced from Moraes et al., 2014]

Considering the brief introduction on the scintillation index $S_{4}$ and its limitations, this work will assume that the signal intensity will be represented by the two parameter $\alpha$ - $\mu$ distribution model (Moraes et al., 2014). The flexibility of this model is evident in Figure 5.5(c), which compares measured occurrences, the best-fit $\alpha-\mu$ distributions (red and blue lines), and the corresponding singleparameter Nakagami- $m$ distribution with $m=1 / S_{4}^{2}=1 / 0.7^{2}=2.04$ (green line).

In this section, a simple model is proposed for simulating equatorial and low latitude transionospheric radio wave scintillation, based on a modified version of 
the one specified by Humphreys et al. (2009). The proposed model will be used to generate sequences of amplitude scintillation $a_{I s c i n t(i, j)}(k)$, using statistical results of amplitude scintillation based on the $\alpha-\mu$ distribution and a representation for the associated power spectral density, assumed to follow that of a low-pass secondorder Butterworth filter.

The model has been implemented as a modified version of the scintillation monitor developed by Humphrey et al. (2009), which originally expects two parameters: (i) dip latitude or $S_{4}$; and (ii) $\tau_{0}$. Figure 5.6 shows the block diagram used for the generation of time series of amplitude scintillation $a_{\operatorname{Iscint}(i, j)}(k)$ according to the modifications introduced by the present work, which additionally assumes the $\alpha-\mu$ distribution for the signal power, thus also requiring its parameters.

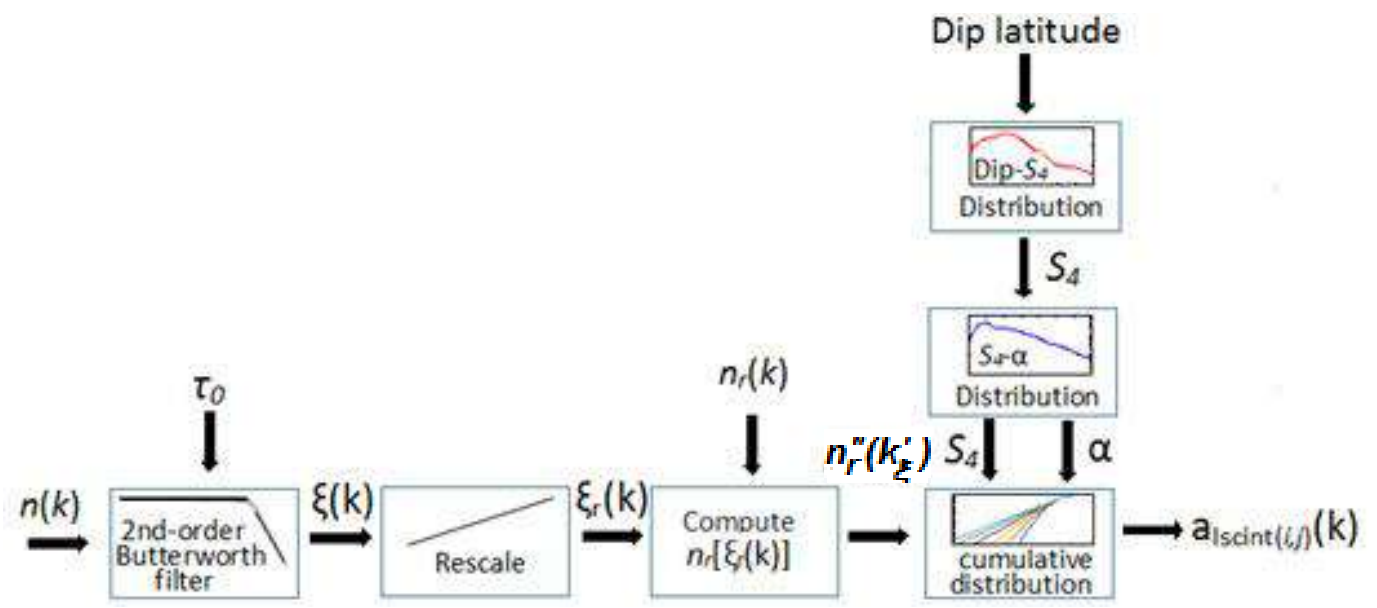

Figure 5.6: Block diagram illustrating a straightforward mechanization of the proposed scintillation model. [Adapted from Humphreys et al. (2009)].

The scintillation simulator is driven by a stationary zero-mean complex white Gaussian noise process $n(k)\left(k=1, \ldots, N_{s}\right.$, where $N_{s}$ is the number of samples) with two-sided power spectral density (PSD) $N o / 2$. In the present work, $N_{s}=3000$ samples that represent a 1-min amplitude scintillation time series at the sampling frequency of $50 \mathrm{~Hz}$. The process $n(k)$ is transmitted through a second-order lowpass Butterworth filter with amplitude response function (Humphreys et al., 2009) that can be represented as

$$
|H(f)|=\frac{1}{\sqrt{1+\left(\frac{f}{f_{n}}\right)^{4}}}
$$


where $f_{n}$ is the filter cut-off frequency

$$
f_{n}=\frac{\beta}{\sqrt{2} \pi \tau_{0}}
$$

where $\beta=1.2396464$ and $\tau_{0}$ is the desired decorrelation time (first delay corresponding to the value of the associated autocorrelation function that equals half of its maximum). According to Humphreys et al. (2009) and Moraes et al. (2014), the Butterworth model also presented good results for long-term analyses, which might be particularly interesting for the estimation of spectral parameters. Figure 5.7 shows a representative power spectral density that is based on data from simulated scintillation $\left(S_{4}=0.6\right.$ and $\left.\tau_{0}=0.2\right)$, and compares this result to the proposed second-order Butterworth model. Note that, for $f \gg f_{n}$, equation (5.36) indicates that $\operatorname{PSD}(f)=|H(f)|^{2} \approx\left(f / f_{n}\right)^{-4}$. The spectral index 4 is higher than values quoted in the literature (Basu et al., 1983; Rino and Carrano, 2011). In principle, it would be possible to generalize equation (5.38) for an arbitrary spectral index if the cutoff frequency $f_{n}$ is additionally adopted as an input variable that substitutes $\tau_{0}$. In this case, equation (5.39), no longer valid, would be unnecessary.

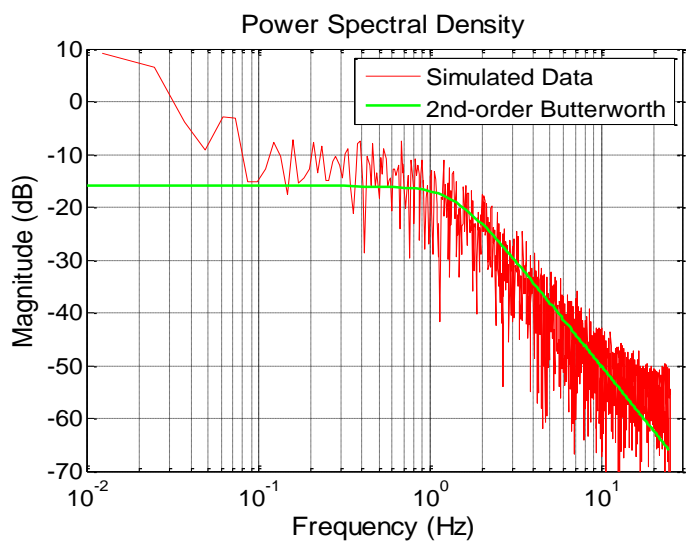

Figure 5.7: Estimated power spectral density from simulated data $\left(S_{4}=0.6\right.$ and $\left.\tau_{0}=0.2\right)$ and the second-order Butterworth model.

After this step, the absolute value of the resulting zero-mean filtered noise process, denoted as $|\xi(k)|$, can be rescaled to the interval $(0,1)$ using the following transformation

$$
\xi_{r}(k)=\frac{|\xi(k)|-\min [|\xi(k)|]}{\max [|\xi(k)|]-\min [|\xi(k)|]}
$$


The obtained rescaled noise $\xi_{r}(k)$ displays values that are not uniformly distributed. Next, sort the elements of the vector $\xi_{r}(k)\left(k=1, \ldots, N_{s}\right)$ in ascending order, simultaneously applying the same operations to the vector of indices $k$. For example, assuming $N_{s}=3$, the vector $\xi_{r}(k)=(0.4,0.6,0.2)$ with vector of indices $k_{\xi}=(1,2,3)$ is transformed into $\xi_{r}^{\prime}(k)=(0.2,0.4,0.6)$ with vector of indices $k_{\xi}^{\prime}$ $=(3,1,2)$ after this procedure. In continuation, generate another array $n_{r}(k)(k=1$, $\left.\ldots, N_{s}\right)$ with uniformly-distributed random numbers in the interval $(0,1)$ and also sort it in ascending order to create the array $n_{r}^{\prime}(k)$. In the final procedure of the central block $n_{r}\left[\xi_{r}(k)\right]$ in Figure 5.6, each element of the resulting filtered noise process is obtained by the equality $n_{r}^{\prime \prime}\left(k_{\xi}^{\prime}\right)=n_{r}^{\prime}(k)$. It should be remarked that the elements of the arrays $n_{r}^{\prime}$ and $n_{r}^{\prime \prime}$ (input to the cumulative distribution block) are also uniformly-distributed random numbers in the interval $(0,1)$.

In the upper block of the vertical branch of the diagram in Figure 5.6, a random value for $S_{4}$ is sorted, according to the probabilities in Table 5.5 for the geomagnetic latitude that is closest to that of the reference station of interest.

Then, for the sorted $S_{4}$, the associated value for $\alpha$ will be selected using the empirical distributions shown in Figure 5.8 for the already selected site. Estimated average values and standard deviations for these distributions and different locations are characterized by Table 5.6.

Table 5.5: Probabilities of $S_{4}$ occurrences in different intervals $\left(S_{4}-0.05\right.$, $\left.\mathrm{S}_{4}+0.05\right)$ and locations for the L1 signal. [Reproduced from Moraes et al., 2018].

\begin{tabular}{|c|c|c|c|c|c|c|c|c|c|}
\hline$S_{4}$ & 0.3 & 0.4 & 0.5 & 0.6 & 0.7 & 0.8 & 0.9 & 1.0 & 1.1 \\
\hline \multicolumn{10}{|c|}{ Fortaleza (Dip latitude $-8.86^{\circ}$ ) } \\
\hline$P_{L 1}$ & 6.27 & 2 & 1 & & 0.31 & 0.12 & 0.05 & 0.04 & 0.0 \\
\hline \multicolumn{10}{|c|}{ Presidente Prudente (Dip latitude $-16.01^{\circ}$ ) } \\
\hline$P_{L 1}($ & 98 & 4.17 & 3.26 & 2.90 & 2.15 & 1.71 & 1.01 & 0.66 & 0.30 \\
\hline \multicolumn{10}{|c|}{ São José dos Campos (Dip latitude $-19.28^{\circ}$ ) } \\
\hline$P_{L 1}(\%)$ & 3.24 & 2.33 & 1.86 & 1.35 & 1.26 & 1.09 & 0.98 & 0.81 & 0.47 \\
\hline \multicolumn{10}{|c|}{ Porto Alegre (Dip latitude $-22.32^{\circ}$ ) } \\
\hline$P_{L 1}(\%)$ & 0.83 & 0.53 & 0.41 & 0.34 & 0.28 & 0.24 & 0.27 & 0.22 & 0.14 \\
\hline
\end{tabular}


Table 5.6: Average values and standard deviations of $\alpha$ in different $S_{4}$ intervals $\left(\mathrm{S}_{4}-0.05, \mathrm{~S}_{4}+0.05\right)$ and locations for the L1 signal measured between 1

November 2014 and 30 March 2015. [Reproduced from Moraes et al., 2018].

\begin{tabular}{|l|l|l|l|l|l|l|l|l|l|}
\hline \multicolumn{1}{|c|}{$\boldsymbol{S}_{\mathbf{4}}$} & $\mathbf{0 . 3}$ & $\mathbf{0 . 4}$ & $\mathbf{0 . 5}$ & $\mathbf{0 . 6}$ & $\mathbf{0 . 7}$ & $\mathbf{0 . 8}$ & $\mathbf{0 . 9}$ & $\mathbf{1 . 0}$ & $\mathbf{1 . 1}$ \\
\hline$E_{L 1}[\alpha] \quad$ Fortaleza & 2.68 & 2.51 & 2.51 & 2.47 & 2.24 & 1.91 & 1.45 & 1.01 & 0.68 \\
\hline$s t d_{L 1}[\alpha]$ Fortaleza & 1.51 & 1.54 & 1.50 & 1.44 & 1.21 & 1.08 & 0.71 & 0.56 & 0.42 \\
\hline$E_{L 1}[\alpha] \quad$ P. Prudente & 2.65 & 2.31 & 2.04 & 2.18 & 2.04 & 1.86 & 1.74 & 1.28 & 1.03 \\
\hline$s t d_{L 1}[\alpha]$ P. Prudente & 1.48 & 1.33 & 1.14 & 1.21 & 1.11 & 0.99 & 0.81 & 0.69 & 0.57 \\
\hline$E_{L 1}[\alpha]$ São José dos C. & 2.78 & 2.44 & 2.48 & 2.52 & 2.61 & 2.17 & 2.25 & 1.49 & 1.02 \\
\hline$s t d_{L 1}[\alpha]$ São José dos C. & 1.63 & 1.60 & 1.42 & 1.40 & 1.28 & 1.19 & 0.92 & 0.80 & 0.61 \\
\hline$E_{L 1}[\alpha] \quad$ Porto Alegre & 3.23 & 2.88 & 2.39 & 2.74 & 2.41 & 2.32 & 1.71 & 1.35 & 1.08 \\
\hline$s t d_{L 1}[\alpha]$ Porto Alegre & 1.78 & 1.63 & 1.38 & 1.23 & 1.41 & 1.01 & 0.80 & 0.72 & 0.51 \\
\hline
\end{tabular}
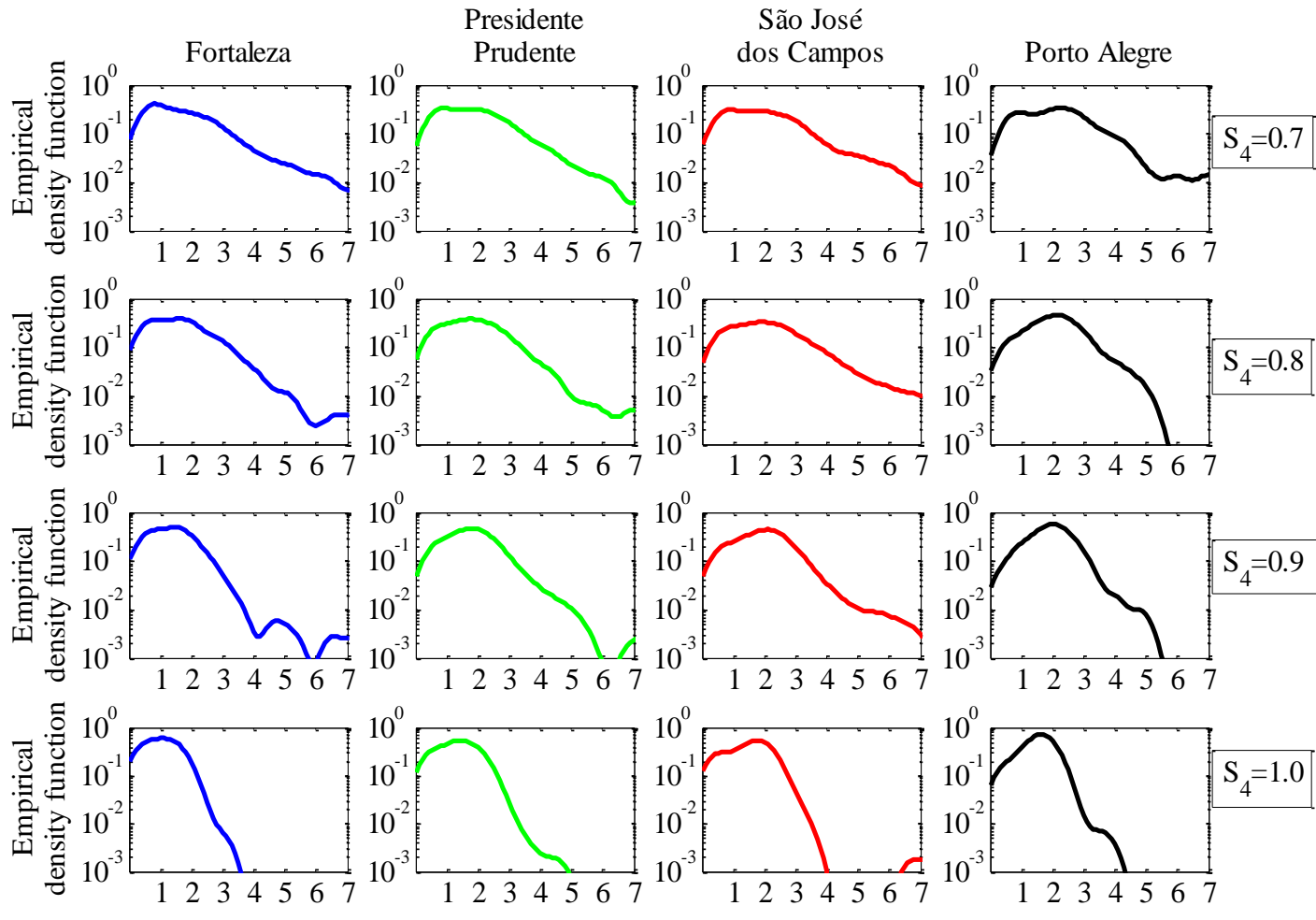

Figure 5.8: Empirical probability density functions of $\alpha$ for different values of $S_{4}$ intervals $\left(S_{4}-0.05, S_{4}+0.05\right)$, locations, and for the GPS L1 signal. [Reproduced from Moraes et al., 2018]

Next, using the sorted values for $S_{4}$ and $\alpha$, the following equation is numerically solved for $\mu$ 
$S_{4}^{2}=\frac{\Gamma(\mu) \Gamma(\mu+4 / \alpha)-\Gamma^{2}(\mu+2 / \alpha)}{\Gamma^{2}(\mu+2 / \alpha)} \rightarrow \frac{\Gamma(\mu) \Gamma(\mu+4 / \alpha)}{\Gamma^{2}(\mu+2 / \alpha)}=S_{4}^{2}+1$

where $\Gamma(x)$ represents the Gamma function.

It is possible to estimate the $\alpha-\mu$ cumulative distribution function, which assumes a normalized envelope $\mathrm{E}[I]=\mathrm{E}\left[r^{2}\right]=1$, using the following expressions

$$
\begin{gathered}
F_{R}(r)=\frac{\gamma\left[\mu,(r / \sqrt{\xi})^{\alpha}\right]}{\Gamma(\mu)}=P\left[\mu,(r / \sqrt{\xi})^{\alpha}\right] \\
\xi=\frac{\Gamma(\mu)}{\Gamma(\mu+2 / \alpha)}
\end{gathered}
$$

where $\gamma(x, y)$ and $P(x, y)$ in expression (5.42) are associated representations of the incomplete Gamma function (Abramowitz and Stegun, 1972) .

Figure 5.9 shows the results of $F_{R}(r)$ as a function of the signal intensity for different values of $\mathrm{S}_{4}$ and $\alpha$ using equation (5.42).
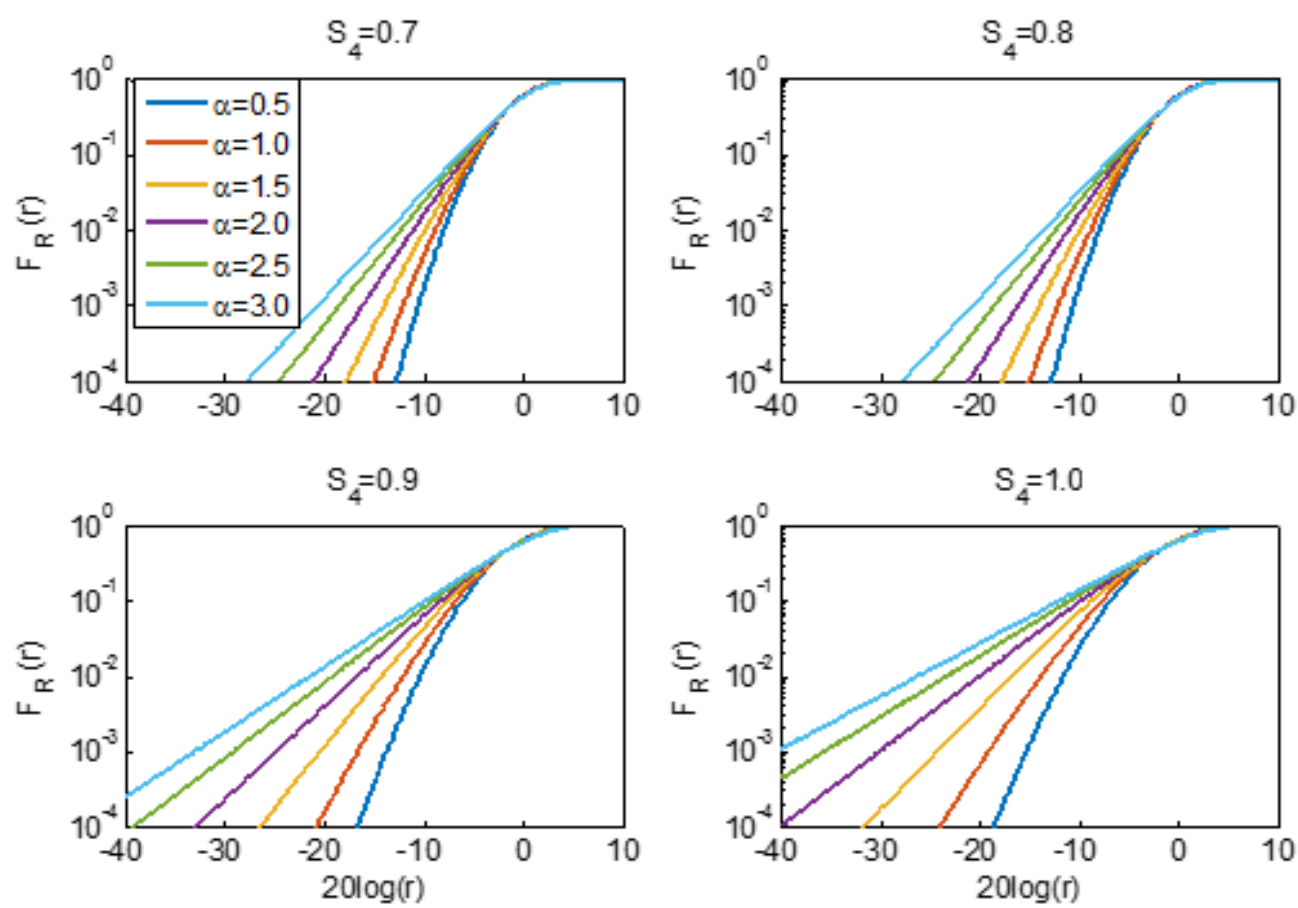

Figure 5.9: Results of $F_{R}(r)$ as a function of the signal intensity, assuming four fixed values of $\mathrm{S}_{4}(0.7,0.8,0.9$ and 1.0$)$ and different $\alpha$ values. 
Obviously, the cumulative distribution function $\mathrm{F}_{\mathrm{R}}(\mathrm{r})$ estimated for each pair $(\alpha, \mu)$ is limited to the interval $(0,1)$. The array of samples $r(k)\left(k=1, \ldots, N_{s}\right)$ representing amplitude scintillation $a_{I S c i n t(i, j)}(k)$ is obtained from

$$
r(k)=F_{R}^{-1}\left[n_{r}^{\prime \prime}(k)\right] \quad k=1, \ldots, N_{s}
$$

Similarly, it is possible to generate time series of $\phi_{\operatorname{Iscint}(i, j)}(k)$ according to the empirical relationships between the sorted the value of $S_{4}$ and $\sigma_{\phi}$ (the standard deviation of phase fluctuations), combined with zero-mean Gaussian cumulative distribution functions displayed in Figure 5.10. Another way is to use the methodology developed by Humphreys et al. (2009).
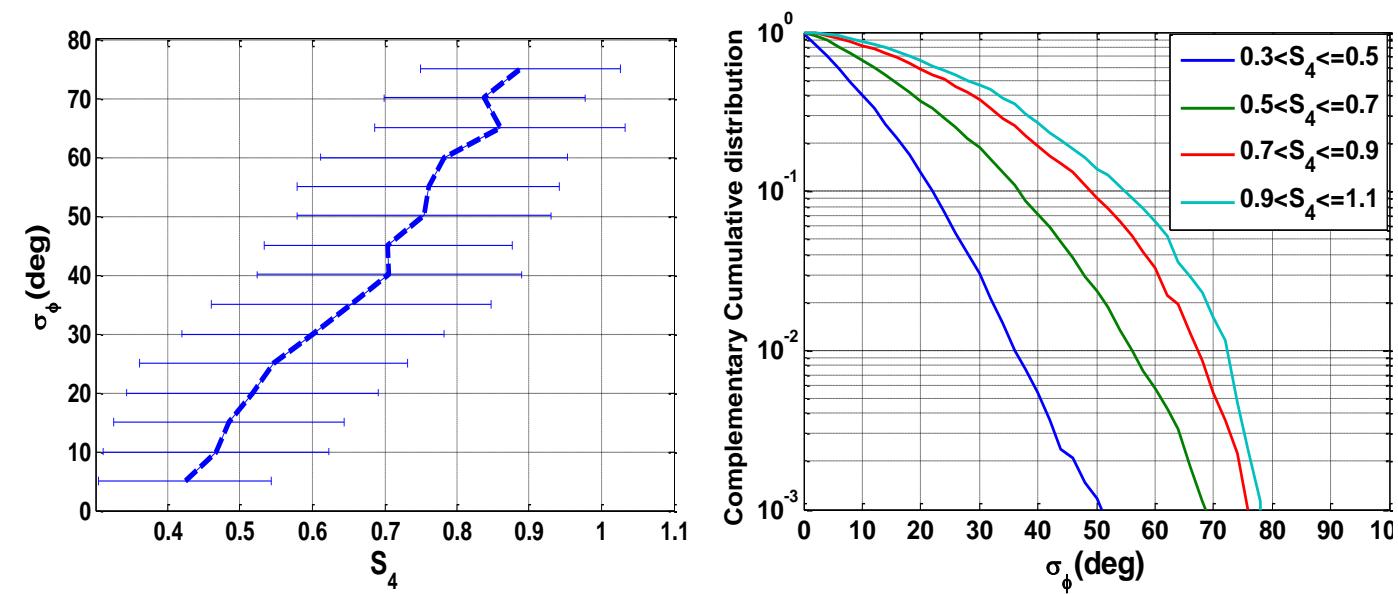

$$
0.3<S_{4}<=0.5 \quad \cdots-0.5<S_{4}<=0.7-0.7<S_{4}<=0.9 \ldots .0 .9<S_{4}<=1.1
$$

Figure 5.10: a) Average values for $\mathrm{S}_{4}$ with standard-deviation bars, as functions of $\sigma_{\varphi}$. b) Complementary cumulative distribution functions of $\sigma_{\varphi}$ for the L1 signal and different $\mathrm{S}_{4}$ ranges. [Reproduced from Moraes et al., 2017]

\subsubsection{Cycle Ambiguity $N_{(i, j)}(k)$}

The carrier phase expression (5.2) displays a cycle ambiguity term $N_{(i, j)}(k)$, which is an integer number. If the signal experiences a loss of lock, the phase measurement has to be reinitiated and a cycle discontinuity may occur. This phenomenon is called cycle slip; that is, the cycle counting has a new beginning due to a signal interruption (Xu, 2007). The consequence of the cycle slips is that 
the adjacent carrier phase observable jumps by an integer number of cycles. Thus, the ambiguity parameter should also be a new one in the related observation model.

The cycle ambiguity can be modeled using statistical results of cycle slips estimated from the residual between the carrier phase and pseudorange measurements from RBMC data. The first step is to detect the cycle slips using the procedure described in the section 3.3.3, then was applied the equation (5.45) to estimate approximate values of ambiguity (assuming that in the previous measurement there is no cycle slip).

$$
N_{1}=\frac{\lambda_{1} \Delta L_{1}-\Delta P_{1}}{\lambda_{1}}
$$

where the operator $\Delta$ indicates the differencing between values associated with the current and the last epochs, $\lambda_{1}$ is the wavelength of the corresponding GPS signal, $L_{1}$ is the received carrier phase observables in units of cycles and $P_{1}$ is the received pseudorange observables in units of meters. Considering the characteristics that the integer ambiguity of each epoch is equal when there is no cycle slip.

The results for each RBMC station can be presented in terms of statistical distributions for the size of the cycle slip and the time interval between consecutive ones. For example, the cumulative distributions (CDFs) and complementary cumulative distributions (CCDFs) of ambiguity value and time between cycle slips for Rio de Janeiro station during the year 2014 are shown in Figure 5.11. This analysis considers different combinations of ranges of the following parameters: (i) EPB season in the Brazilian sector, closely associated with that of ionospheric scintillation; and (ii) solar activity, represented by the F10.7 index described in Table 5.7. Note that September to March means January to March 2014 and September to December 2014. 
Table 5.7: Season and solar activity parameters considered for cycle ambiguity.

\begin{tabular}{|lll|}
\hline Season: & $\begin{array}{l}\text { April }- \text { August } \\
\text { September }- \text { March }\end{array}$ & AA \\
& SM \\
\hline Solar activity (F10.7 index): & F10.7 $<100$ & LO \\
& $100 \leq \mathrm{F} 10.7<150$ & MD \\
& F10.7 $\geq 150$ & HG \\
\hline
\end{tabular}
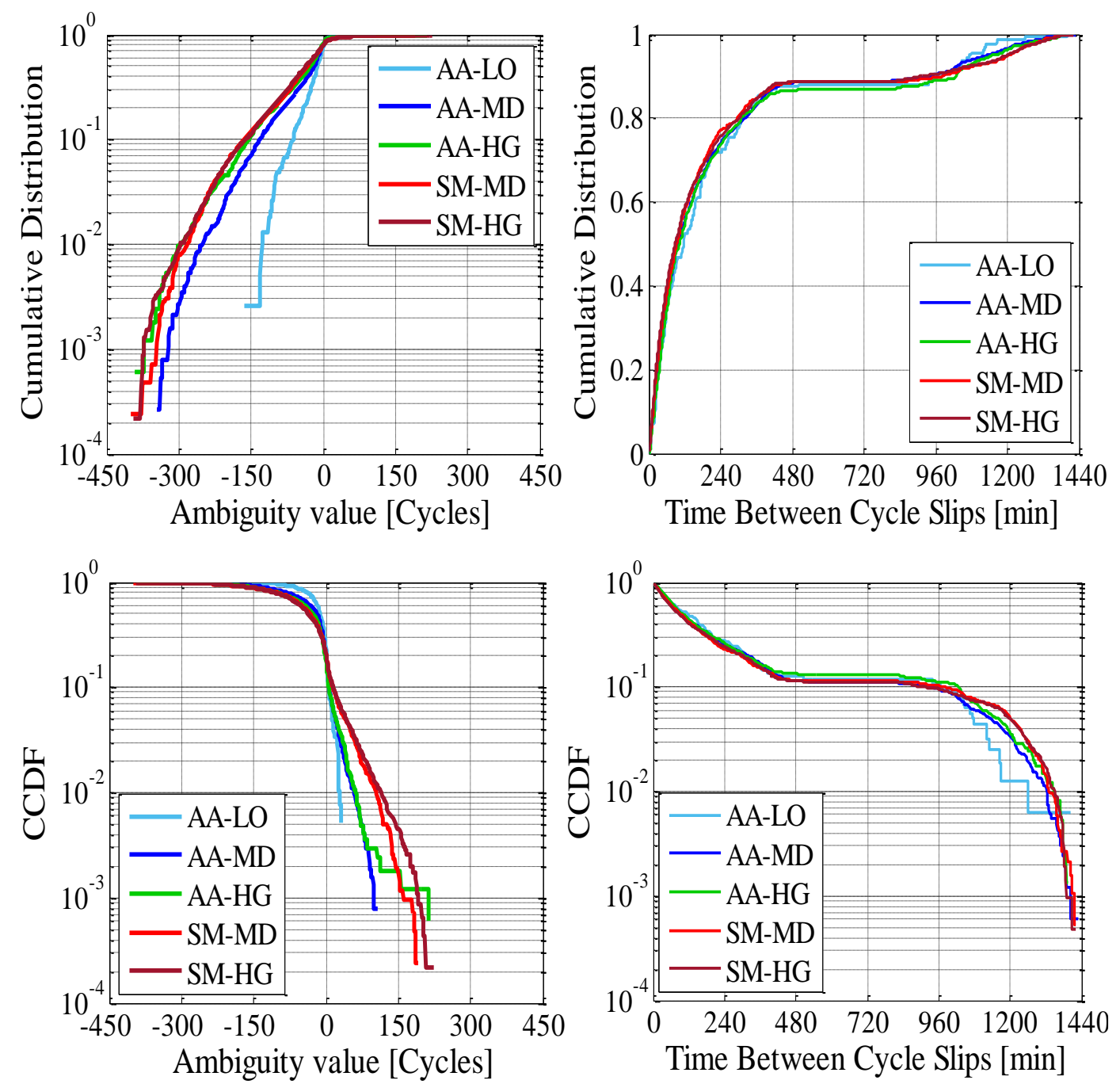

Figure 5.11: Cumulative distribution function and complementary cumulative distribution function of ambiguity value (left); and cumulative distribution function and complementary cumulative distribution function of time between consecutive cycle slips (right) for the Rio de Janeiro RBMC station during the year 2014.

The left panels in Figure 5.11(top and bottom) show that the ambiguity values (size of cycle slips) estimated for days with low activity between April to August 
(AA-LO) are small in comparison to cycle slips estimated for days with high activity between September to March (SM-HG), AA-LO depict values between -159 cycles and 33 cycles; (SM-HG) present values between -398 cycles and 224 cycles. The right panels in Figure 5.11 shows that the cumulative distribution of occurrence time between two consecutives cycle slips are similar because the normalized occurrence values are concentrated in the same interval of time (1 min to $480 \mathrm{~min}$ ). It is important to remark that values of the F10.7 index less than 100 sfu did not occur from September to March during the year 2014. For this reason, this combination is not present in the above statistical results.

The occurrence of the two parameters of the cycle slip model (size and time interval) can be simulated using the estimated cumulative distribution functions for the corresponding season and F10.7 index represented in Figure 5.11, in association with uniformly-distributed random number generators, as described in Section 5.1.6.

\subsubsection{Effective Isotropic Radiated Power $\operatorname{EIRP}_{(i, j)}(k)$}

The effective isotropic radiated power of the GPS L1 signal combines the transmitter power and losses with the satellite antenna gain in the receiver direction, being expressed as

$$
\operatorname{EIRP}_{(i, j)}(k)=P_{t}-l_{t p(i)}+G_{T(i, j)} \quad[\mathrm{dBW}]
$$

where $P_{t}[\mathrm{dBW}]$ is the transmitter power; $l_{t p(i)}[\mathrm{dB}]$ is the power loss along the transmitter chain; and $G_{T(i, j)}[\mathrm{dBi}]$ is the satellite antenna gain in the receiver direction. These parameter values, which vary according to the block of satellites available in space, will be specified in subsection 5.2.

\subsubsection{Receiver Clock Errors $\Delta t_{r(j)}(k)$ and Random Errors $v_{(i, j)}(k)$}

GPS signals are delayed as they propagate through the receiver chain. Additionally, there are oscillators that operate with low resolution, causing further signal delays. The receiver clock error and the random errors are modeled based on error budgets available in the literature, using Gaussian random variables with zero 
mean and standard deviation on the order of $0.5 \mathrm{~m}$ and $3.0 \mathrm{~m}$, respectively (Seeber, 2003; Hoffman-Wellenhof et al., 2008; Monico, 2008).

\subsection{Results from the Pseudorange, Carrier Phase and Received Signal Models}

This section presents estimated results from the developed model for pseudorange $P R_{(i, j)}(k)$, carrier phase $\phi_{(i, j)}(k)$ and received signal $C_{(i, j)}(k)$, considering each component of these observables, described in the previous sections. The results are presented as time series for a specific case.

Case study: Rio de Janeiro, 19 - 20 December 2014 event between 21:00 and 05:00 UTC

These observables were simulated for channels defined by different satellites (i) and the Rio de Janeiro receiver station (j) between 21:00 UTC of 19 December 2014 and 05:00 UTC of 20 December 2014, with time denoted by $(k)$. The positions of each satellite are determined at the receiver time, using the broadcast navigation file. Figure 5.12 shows the position of the Rio de Janeiro station (red dot) and RBMC stations (blue dots), while the red curve represents the geomagnetic equator.

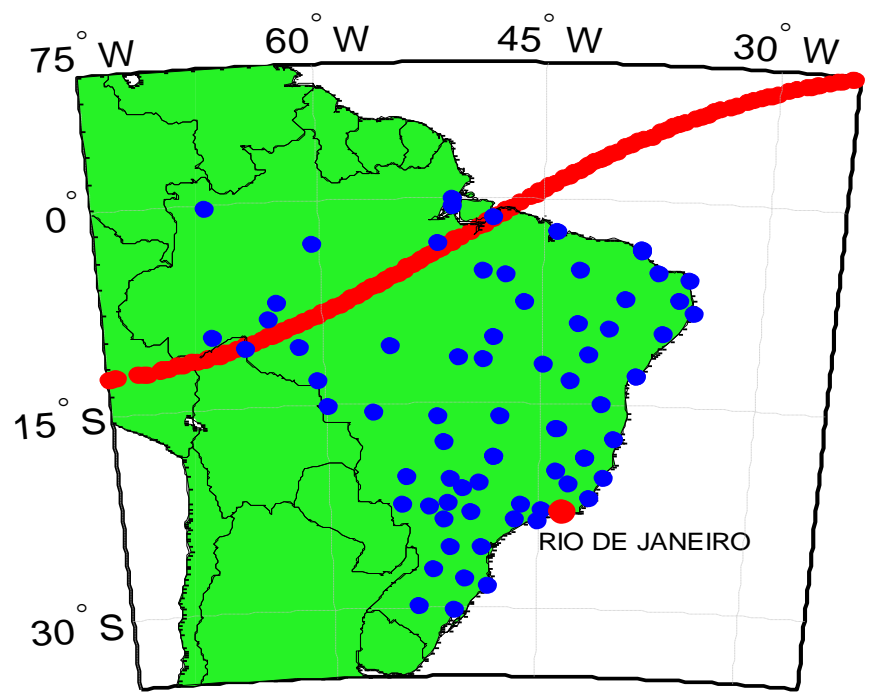

Figure 5.12: Positions of the Rio de Janeiro receiver (red dot) and RBMC stations (blue dots), as well as the geomagnetic equator (red curve). 
The geophysical parameters, description of the Rio de Janeiro station and simulation system parameters used in the simulation are described in Tables 5.8, 5.9, and 5.10.

Table 5.8: Geophysical parameters on 19-20 December 2014.

\begin{tabular}{|c|c|}
\hline Parameter & Value \\
\hline DoY & $353-354$ \\
\hline F10.7 index & $208.9-196.7[\mathrm{sfu}]$ \\
\hline Kp index & $2-2.7-2.3$ \\
\hline
\end{tabular}

Table 5.9: Description of the Rio de Janeiro station.

\begin{tabular}{|c|c|c|}
\hline \multirow{3}{*}{$\begin{array}{c}\text { Location } \\
\text { Description }\end{array}$} & Parameter & Value \\
\cline { 2 - 3 } & Latitude & $-22.82^{\circ}$ \\
\hline \multirow{3}{*}{ Environment } & Longitude & $-43.30^{\circ}$ \\
\hline & Dip latitude & $-20.83^{\circ}$ \\
\cline { 2 - 3 } & Air pressure & $1012[\mathrm{hPa}]$ \\
\cline { 2 - 3 } & Temperature & $25^{\circ} \mathrm{C}$ \\
\cline { 2 - 3 } & Dielectric constant $\varepsilon_{r}($ wet ground) & $30 \%$ \\
\cline { 2 - 3 } & Conductivity $\sigma$ & 30 \\
\cline { 2 - 3 } & Height receiver antenna & $0.20 \mathrm{~S} / \mathrm{m}$ \\
\cline { 2 - 3 } & Humidity \\
\hline
\end{tabular}

Table 5.10: Simulation system parameters.

\begin{tabular}{|c|c|c|}
\hline \multirow{4}{*}{$\begin{array}{c}\text { System } \\
\text { Description }\end{array}$} & Parameter & Value \\
\cline { 2 - 3 } & Losses in transmitter path & $16.25 \mathrm{dBW}$ \\
\cline { 2 - 3 } & Gain of transmitter antenna & $13.25 \mathrm{~dB}$ \\
\cline { 2 - 3 } & Gain of receiver antenna & $3 \mathrm{~dB}$ \\
\cline { 2 - 3 } & GPS C/A code chip length & $293 \mathrm{~m}$ \\
\hline
\end{tabular}




\subsubsection{Geometric Distance $\rho_{(i, j)}(k)$}

The geometric distances between GPS satellites $(i)$ and the Rio de Janeiro station $(j)$ were estimated using equation (5.5), in combination with the satellite position resulting from the iterative method described in Appendix A at receiver time $(k)$. During the described time interval, the Rio de Janeiro receiver tracked 16 satellites listed in the legend of Figure 5.13. The estimated values of geometric distances for each satellite-receiver pair are displayed using continuous or dashed lines of the same color, for satellite elevations (from the station) greater or less than $20^{\circ}$, respectively.

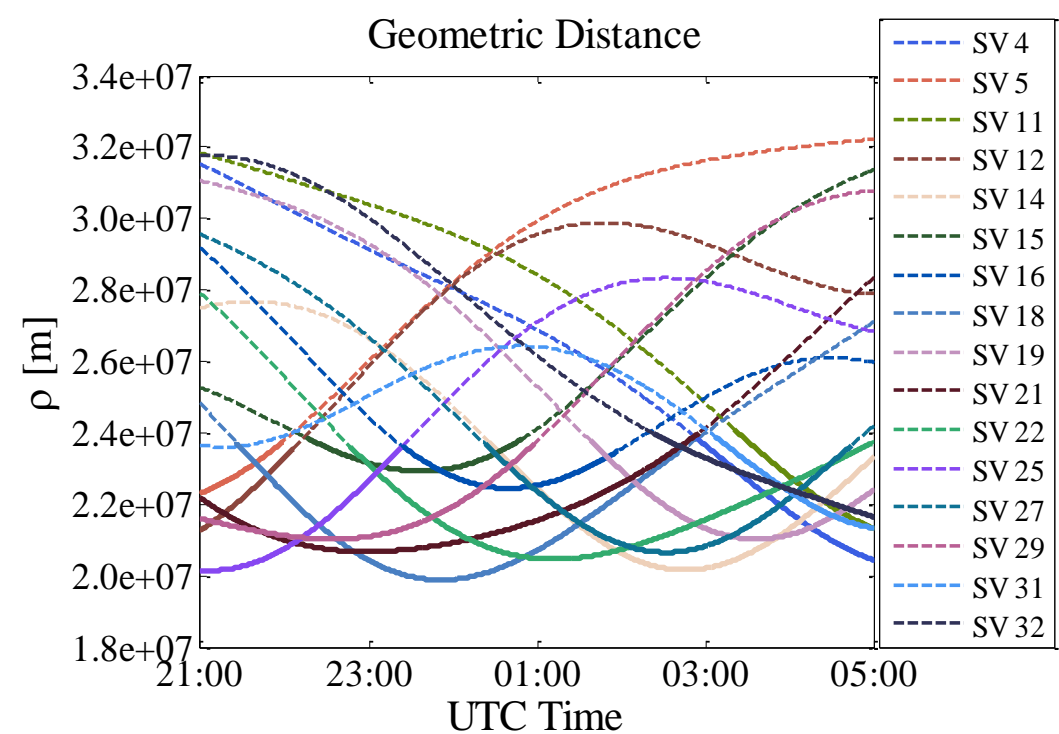

Figure 5.13: Estimated geometric distances between the Rio de Janeiro station and GPS satellites on the night of 19-20 December 2014. Continuous or dashed lines of the same color are used for satellite elevations (from the station) greater or less than $20^{\circ}$, respectively.

The visibility interval of each satellite (for the elevation mask of $20^{\circ}$ ) varies between 2.5 and 5.0 hours approximately, as shown in Figure 5.13. For example, satellites SV15 and SV18 are visible from 22:16:15 to 00:49:30 UTC and from 21:24:00 to 02:40:15 UTC, respectively, considering the elevation mask. For each curve, the minimum value of the geometric distance indicates the closest point of the satellite from the station, the associated Coordinated Universal Time, as well as the position with the maximum elevation angle. 
The elevation angle $\varepsilon_{(i, j)}(k)$ of the satellites from the Rio de Janeiro station is an important parameter used in mapping functions to calculate the multipath, ionospheric and tropospheric delays. It can be calculated using the satellite and receiver position at the reception time. The estimated values of the elevation angle from the Rio de Janeiro station to each visible satellite during the specified interval are shown in Figure 5.14. The horizontal line at $20^{\circ}$ represents the elevation mask used in this work.

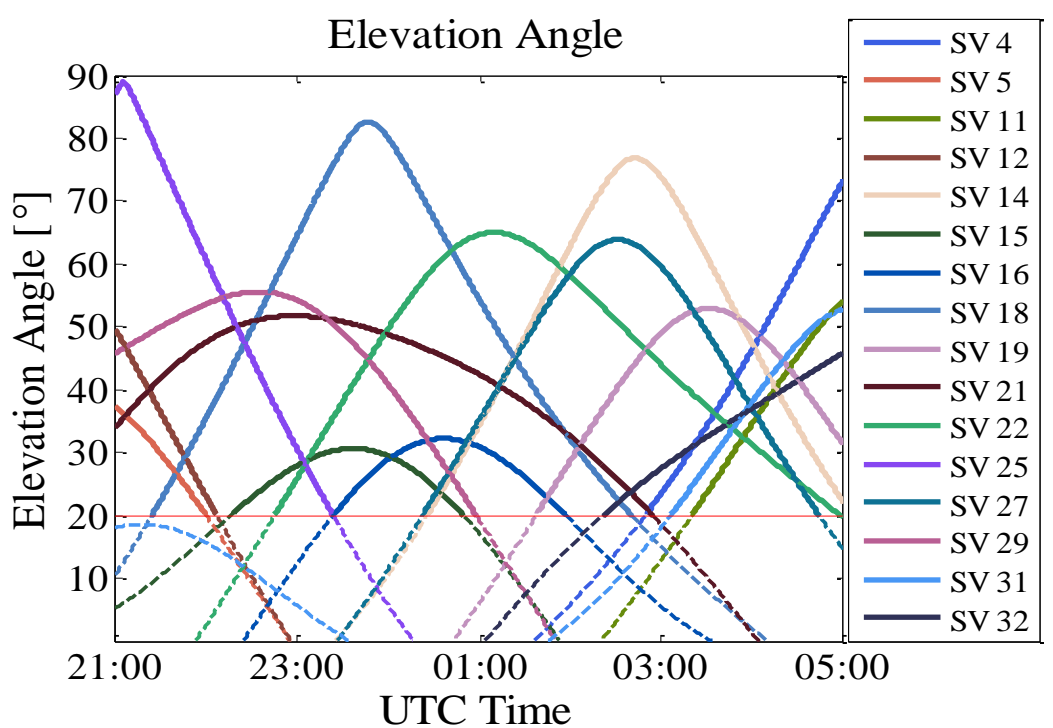

Figure 5.14: Estimated elevation angle from the Rio de Janeiro station to each visible GPS satellites on the night of 19-20 December 2014. The horizontal line at $20^{\circ}$ represents the elevation mask used in this work.

Figures 5.13 and 5.14 indicate that, for the circular GPS orbits during the test case interval, the elevation angle increases when the distance decreases. For example, the violet curves for the estimated SV25 geometric distance and elevation angle reach the respective extreme values 20144655.1 meters and $88.93^{\circ}$ at 21:05:15 UTC. The intersection between each elevation curve and the elevation mask line indicates the interval of time that will be considered for the corresponding satellite in future results in this Section.

\subsubsection{Satellite Clock Error $\Delta t_{S(i)}(k)$}

Since the satellite oscillators are not perfectly synchronized to the GPS time, the satellite clock error is one of the main components that affect the positioning 
accuracy. Using the procedures described in Subsection 5.1.2, the estimated values of the satellite clock error for each visible GPS satellite tracked by the Rio de Janeiro receiver are shown in Figure 5.15.
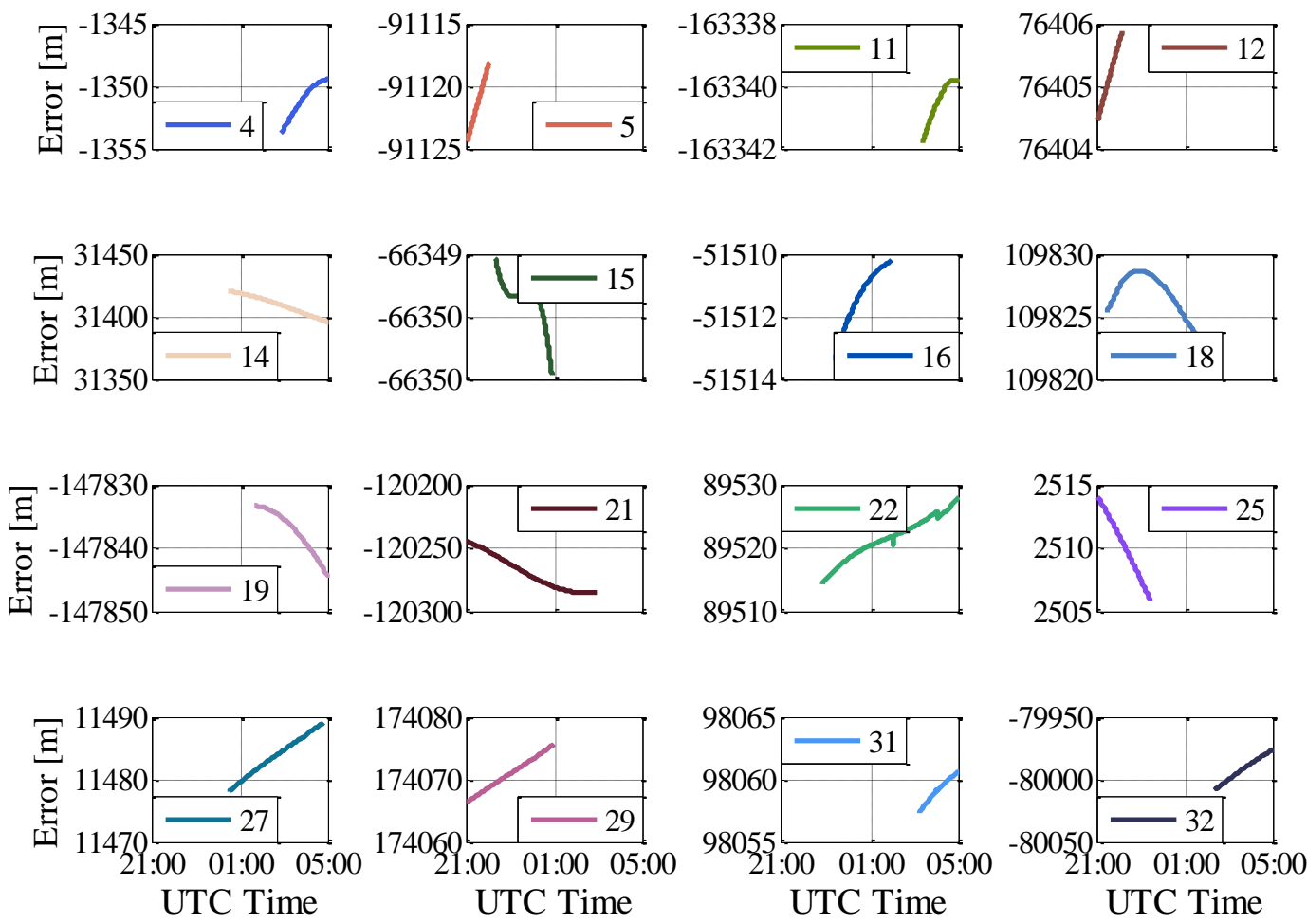

Figure 5.15: Estimated satellite clock error for each GPS satellite tracked by Rio de Janeiro station on the night of 19-20 December 2014.

Positive errors in Figure 5.15 indicate that the satellite clock is advanced with respect to the system time. That is, the signal transmission time from the satellite will be marked later than what it actually is. The Panels in Figure 5.15 also reveal that the satellite clock errors present differences within the selected time interval. For example, SV04 presents clock errors that vary from 109825.3 meters to 109821.5 meters between 21:24:00 to 02:40:15 UTC. From these results it is evident that the satellite clock errors have a major impact on the pseudoranges.

\subsubsection{Ionospheric Delay $I_{(i, j)}(k)$}

The ionospheric delay is estimated using the model that adds IRI model 2016 predictions to the average VTEC residual obtained by RBMC measurements, as described in Subsection 3.6.6, considering the corresponding geophysical 
parameters for each IPP. This result is then combined with the mapping function presented in Subsection 5.1.3. The final results for the satellite-receiver pairs are shown in Figure 5.16.

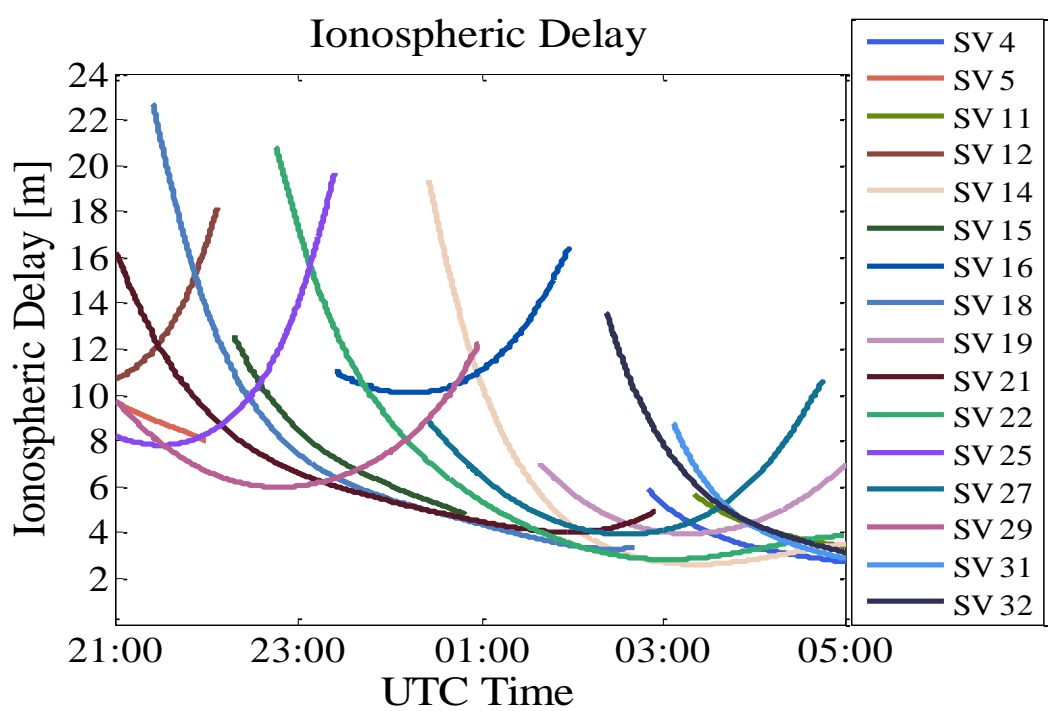

Figure 5.16: Estimated ionospheric delays between GPS satellites and the Rio de Janeiro station on the night of 19-20 December 2014.

The magnitude of these delays is determined by the state of the ionosphere during signal propagation. The ionospheric delay presents highly variable spatial and temporal variations, as described in Section 3.1. For example, the SV14 and SV18 signals present ionospheric delays respectively equal to $2.6 \mathrm{~m}$ around 03:23:00 UTC and to $22.7 \mathrm{~m}$ around 21:24:00 UTC. The variation of the ionospheric delay depends on its state, as well as on the elevation angle. Indeed, the signal path transits through a larger extent of the ionosphere at lower elevation angle. In addition, the ionospheric delay presents variation with seasonal and other geomagnetic parameters. The results for a winter night with low solar activity (night of 21-22 July 2014 , F10.7 = $93.0 \mathrm{sfu}$ ) for the Rio de Janeiro station are shown in Figure 5.17.

Figure 5.17 reveals that the SV17 and SV28 signals present ionospheric delays equal to 15.9 meters about 22:50:00 and 2.6 meters about 05:00:00 UTC, respectively. It is noted that the maximum ionospheric delay observed in Figure 5.16 (for a December night with high solar flux activity) is approximately $6.8 \mathrm{~m}$ greater than that in Figure 5.17 (for a July night with a moderate solar flux activity). 


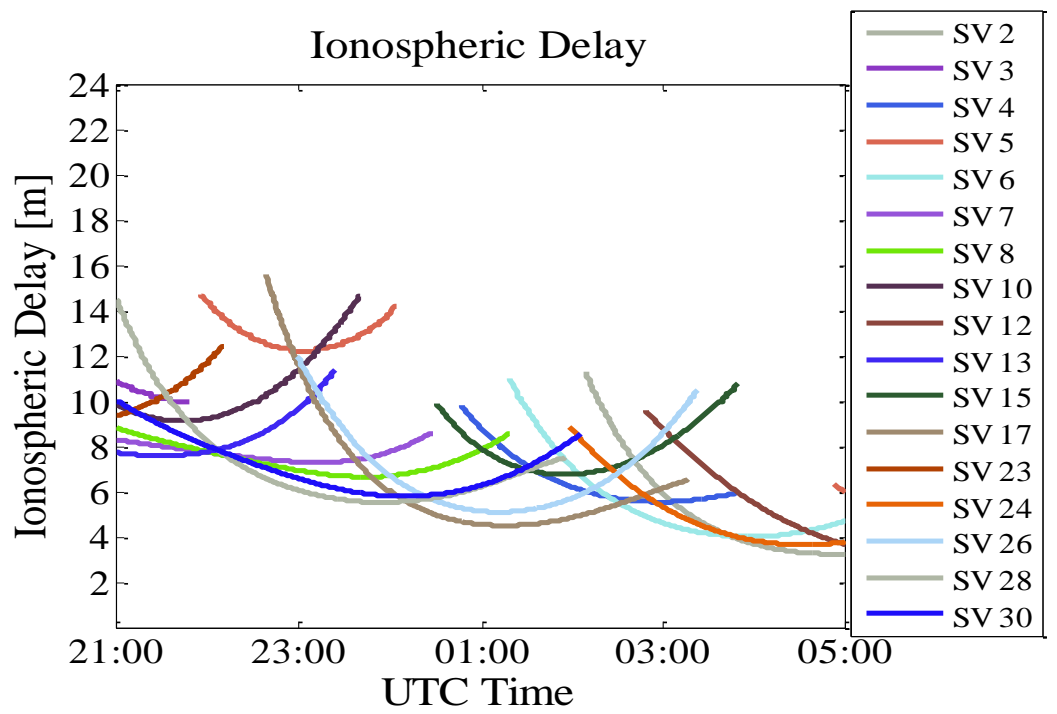

Figure 5.17: Estimated ionospheric delay between GPS satellites and the Rio de Janeiro Station on the night of 21-22 July 2014.

\subsubsection{Tropospheric Delay $T_{(i, j)}(k)$}

Considering the environmental parameters of the Rio de Janeiro station described in Table 5.9, the time interval information of visibility and elevation angle estimated from the Rio de Janeiro station to each GPS satellite, is possible to estimate the corresponding tropospheric delay using the model presented in Subsection 5.1.4. The estimated values for the different satellite-station pairs are shown in Figure 5.18.

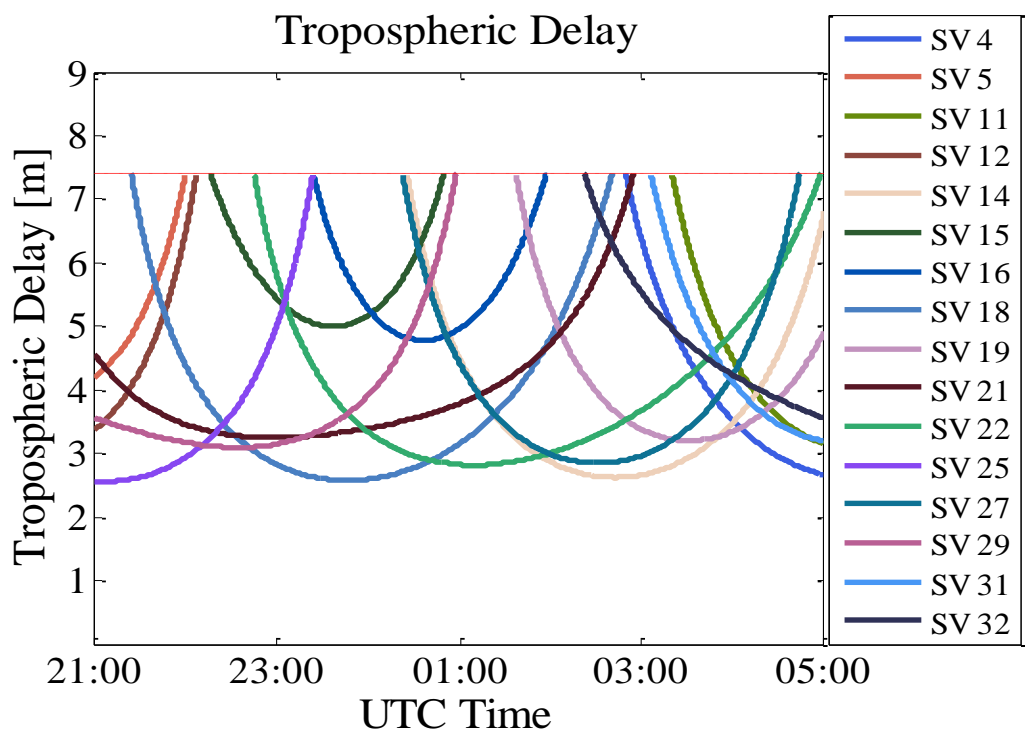

Figure 5.18: Estimated tropospheric delay between GPS satellites and the Rio de Janeiro station on the night of 19-20 December 2014. 
Figure 5.18 reveals that the estimated tropospheric delays for all satellites vary from a minimum of 2.5 meters for SV 25 at 21:05:15 UTC, when the elevation angle is $88.93^{\circ}$, to $7.3 \mathrm{~m}$ for all satellites. This maximum value has been obtained when elevation angles reach the lower threshold of $20^{\circ}$ of the elevation mask. Similarly, the variation of the tropospheric delay depends on elevation angle, which affects the path extension within the neutral atmosphere.

\subsubsection{Multipath $\boldsymbol{m}_{(i, j)}(k)$}

The multipath effects due to ground reflections on the carrier phase $m_{\varphi(i, j)}(k)$, received signal power $m_{C(i, j)}(k)$, and receiver code $m_{P R(i, j)}(k)$ were estimated using equations (5.33) to (5.36) of Subsection 5.1.5. These multipath errors were computed using the surface and system parameters described in Table 5.8 and 5.9, respectively. Figure 5.19 shows the computed code, carrier phase and amplitude multipath errors, respectively.

The first panel of Figure 5.19 shows the pseudorange multipath delay. Fast and relatively small oscillations are observed for each satellite-receiver pair, asymmetrically located with respect to the zero reference. This delay component depends on the elevation angle, reflection coefficient, as well as on the chip length. For example, the maximum absolute error estimated for SV5 is 0.32 meters.

The second and third panels of Figure 5.19 display results of carrier phase multipath delay and multipath effects on the received signal power, respectively. Both present fast and small-amplitude oscillations symmetrically located with respect to the corresponding zero reference. These components also depend on the elevation angle and reflection coefficient. It is important to note that the estimated carrier phase multipath errors are minimal in comparison to those from the pseudorange multipath component. For example, the maximum carrier phase multipath delay estimated for SV5 is $0.48 \mathrm{~mm}$. It is also noted that the maximum estimated fading in the received signal due to multipath for SV5 and SV12 are 1.32 $\mathrm{dB}$ and $0.40 \mathrm{~dB}$, respectively. 

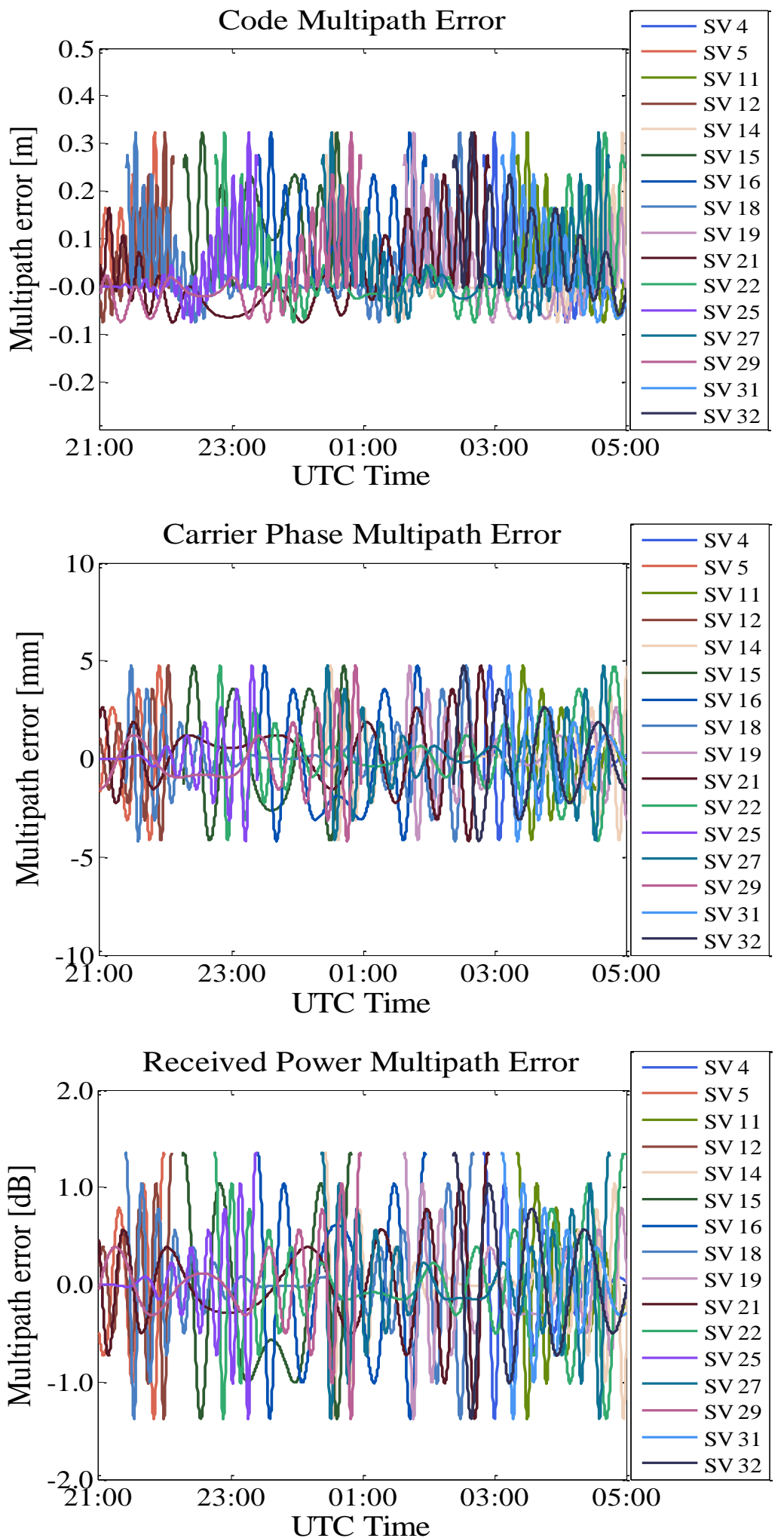

Figure 5.19: Estimated code (top), carrier phase (middle), and received power (bottom) multipath errors due to one rough plane reflector for GPS L1 signals, considering a two-ray model. 


\subsubsection{Amplitude Scintillation $a_{I s c i n t(i, j)}(k)$}

The left Panels of Figure 5.20 show 1-min time series representing simulated amplitude scintillation using the $\alpha-\mu$ distribution model for satellites SV14, SV16, SV18, SV21, SV12 and SV27, assuming the $\left(S_{4}, \alpha, \mu\right)$ values displayed in the associated legends (two-time series in each Panel). These results were simulated for the Rio de Janeiro station (dip latitude $-20.83^{\circ}$ ) using the São José dos Campos statistical distribution, since they are located at close dip-latitude range. The experimental records associated with time interval 01:00 UTC to 01:01 UTC on 20 December 2014 were used to assign the displayed values of $S_{4}$ and the sampling frequency is $50 \mathrm{~Hz}$. The right panels in Figure 5.20 show the associated power spectral density for each channel.
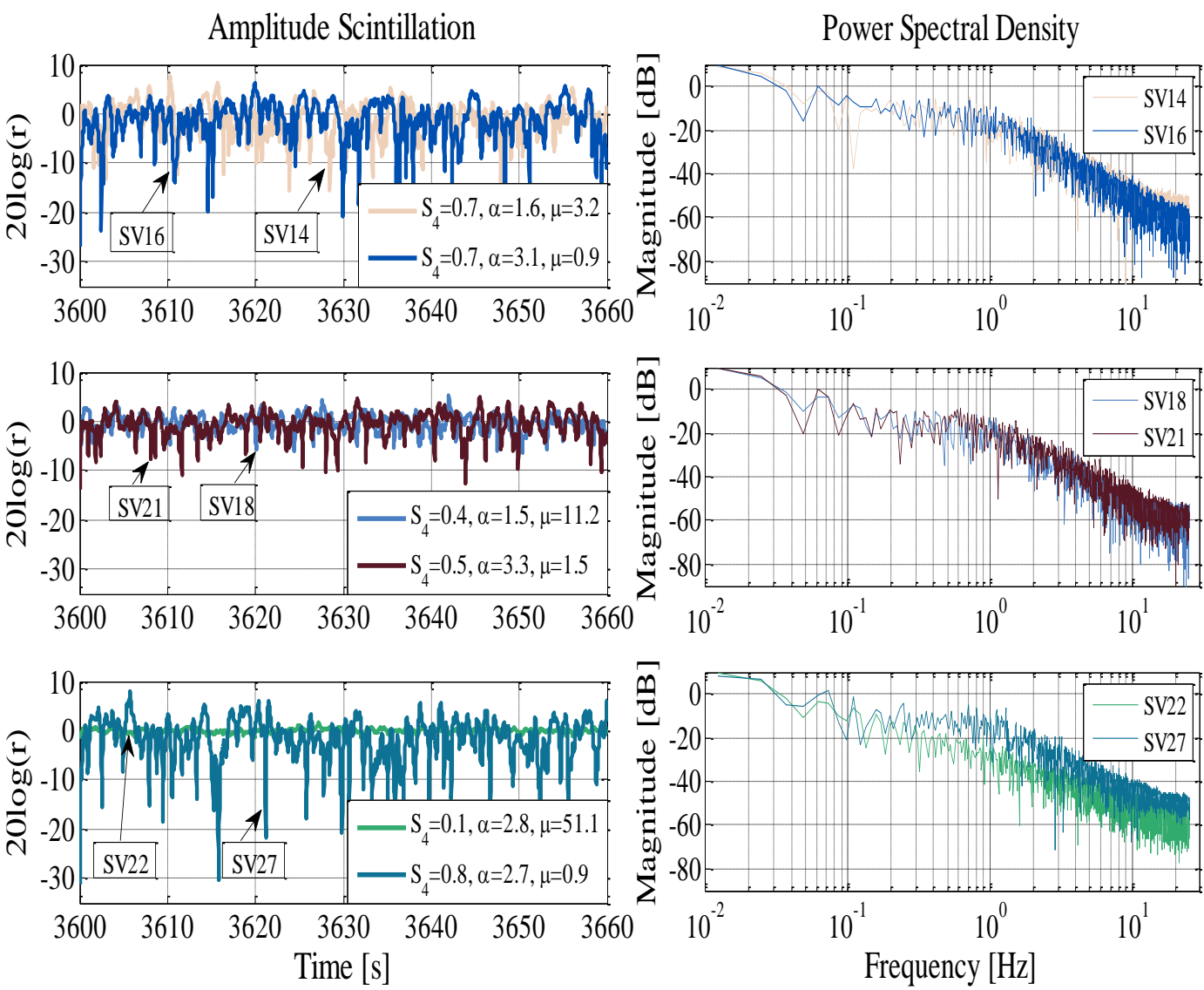

Figure 5.20: (Left column) one-min time series of amplitude scintillation simulated for the Rio de Janeiro station and satellites SV14, SV16, SV18, SV21, SV22, and SV27 during the time interval 01:00 UTC to 01:01 UTC on 20 December 2014, assuming the $\left(\mathrm{S}_{4}, \alpha, \mu\right)$ values displayed in the associated legends; (Right column) power spectral densities of the corresponding amplitude scintillation. 
The three left Panels of Figure 5.20 show that the occurrences and depths of deep fades are very different for the six signals. For example, the first Panel shows that the fading depths for satellites SV14 and SV16 reached $18.5 \mathrm{~dB}$ (for $\mathrm{S}_{4}=0.7$ and $\alpha=1.6$ ) and $24.5 \mathrm{~dB}$ (for $S_{4}=0.7$ and $\alpha=3.1$ ), respectively. This Panel shows two signals with the same $S_{4}$ index, indicating that the increased $\alpha$ value represents a more severe scenario for propagation, with a higher occurrence of deep fading of the received signal. On the other hand, fadings greater than $8.0 \mathrm{~dB}$ and $14.1 \mathrm{~dB}$ are observed for SV18 $\left(S_{4}=0.4\right.$ and $\left.\alpha=1.5\right)$ and SV21 $\left(S_{4}=0.5\right.$ and $\left.\alpha=3.3\right)$ in the second Panel, respectively. Finally, the lower Panel of Figure 5.20 shows two contrasting cases of weak and strong scintillation: fading in the SV22 and SV27 signals reached $1.8 \mathrm{~dB}$ (for $\mathrm{S}_{4}=0.1$ and $\alpha=2.8$ ) and $36.2 \mathrm{~dB}$ (for $\mathrm{S}_{4}=0.8$ and $\alpha=$ $2.7)$, respectively.

The right Panels of Figure 5.20 plot the power spectral densities derived from the corresponding 1-min time series of simulated amplitude scintillation. As expected, the plots of the power spectral density in the selected axes display the decreasing straight line for frequencies in excess of the Fresnel frequency (Singleton, 1962; Banola et al., 2005; Rino, 2011). The results also show that the power spectral densities increase with $\mathrm{S}_{4}$.

The frequency distribution of simulated data and theoretical $\alpha-\mu$ probability density functions corresponding to the simulated time series of amplitude scintillation for each channel are shown in Figure 5.21.

It is noted from Figure 5.21 that differences between the distributions estimated from the simulated data are confirmed by the $\alpha-\mu$ model for the corresponding parameters. Furthermore, it is observed that, for a fixed $\mathrm{S}_{4}$, the deepfading section of the distribution tends to rise as the value of $\alpha$ increases, suggesting that fading events are most likely to occur. 

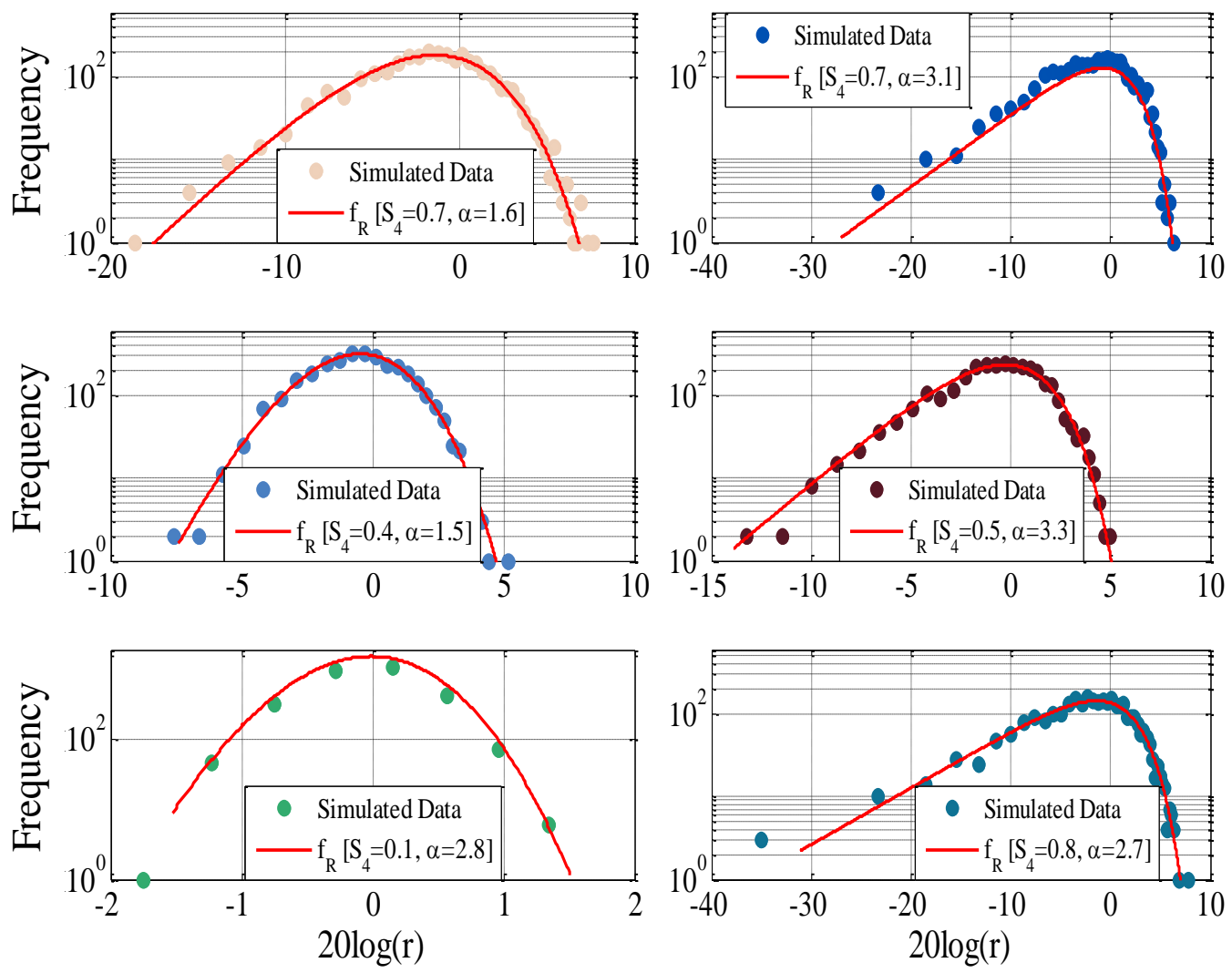

Figure 5.21: Frequency density function of simulated data and theoretical $\alpha$ $\mu$ probability density functions for the simulated time series of amplitude scintillation for each channel specified in association with Figure 5.20.

The next simulation of 1-min time of series of amplitude scintillation is based on the Fortaleza station (dip latitude $-8.86^{\circ}$ ), located at a different latitude than Rio de Janeiro station (dip latitude $-20.83^{\circ}$ ). The results of amplitude scintillation, using the corresponding $\mathrm{S}_{4}$ values for the same time interval, are shown in Figure 5.22.

The left Panels of Figure 5.22 present results amplitude scintillation simulated for the Fortaleza station that display shallower fadings in comparison to the values predicted for the Rio de Janeiro station in Figure 5.20 for most of the satellites. This is mostly due to the different positions of the two stations in relation to the geomagnetic equator, which tend to yield smaller values of $S_{4}$ at the former. For example, fade depths in SV14 transmissions reached $11.9 \mathrm{~dB}$ and $24.5 \mathrm{~dB}$ in Fortaleza and Rio de Janeiro, respectively. 

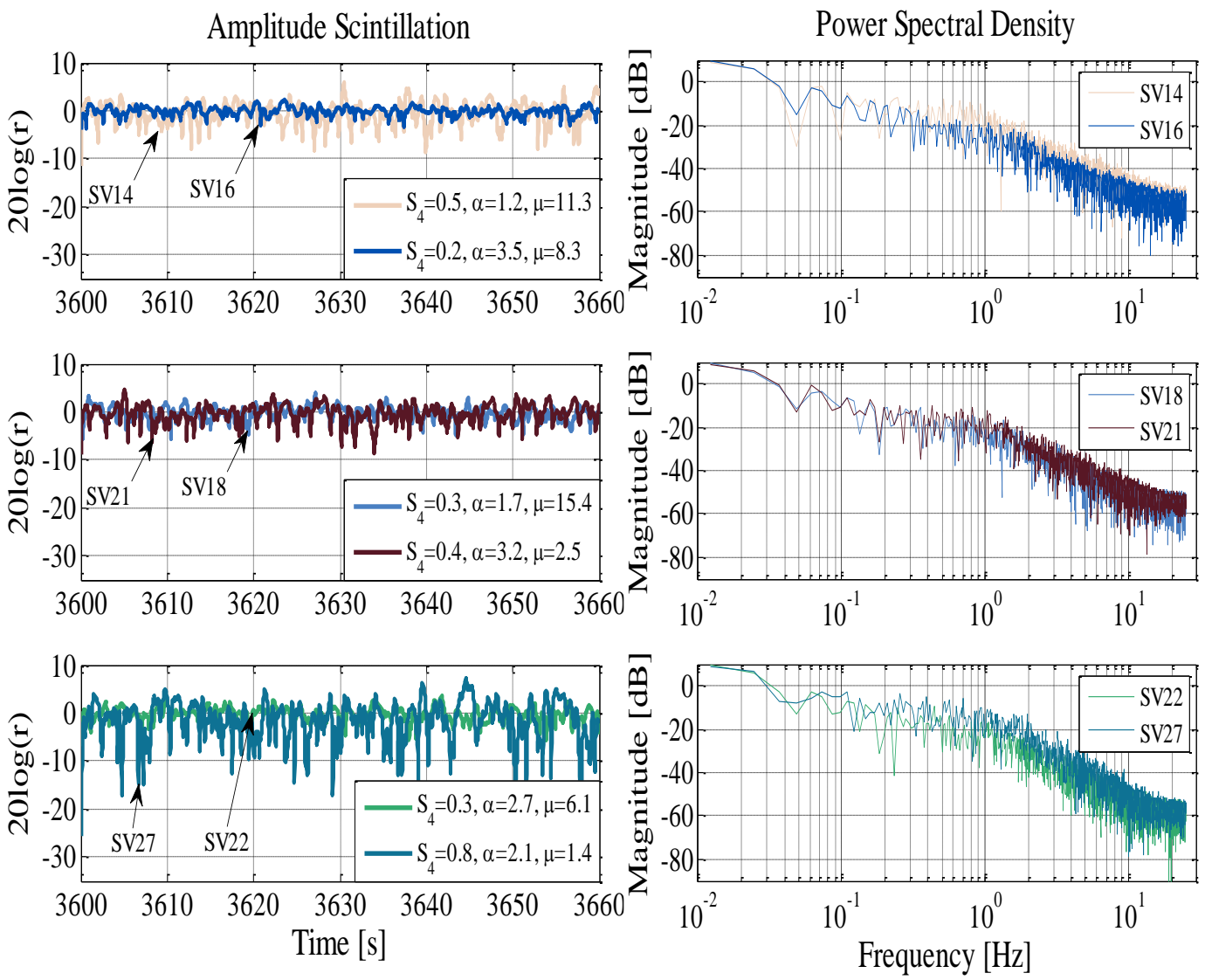

Figure 5.22: (Left column) one-min time series of amplitude scintillation simulated for the Fortaleza station and satellites SV14, SV16, SV18, SV21, SV22, and SV27 during the time interval 01:00 UTC to 01:01 UTC on 20 December 2014, assuming the $\left(\mathrm{S}_{4}, \alpha, \mu\right)$ values displayed in the associated legends; (Right column) power spectral densities of the corresponding amplitude scintillation.

Additionally, 1-min time of series of amplitude scintillation were simulated for Rio de Janeiro station (Dip latitude $-20.83^{\circ}$ ) during the time interval 01:00 UTC to 01:01 UTC, but on 22 July 2014. The amplitude scintillation results are shown in Figure 5.23.

Figure 5.23 shows that all satellite signals present weak scintillation. For example, the deepest fading of SV26 is $3.9 \mathrm{~dB}$. The results displayed in Figure 5.21 and Figure 5.23 reflect the seasonal effect of scintillation for the Rio de Janeiro station, more intense from September to March. 

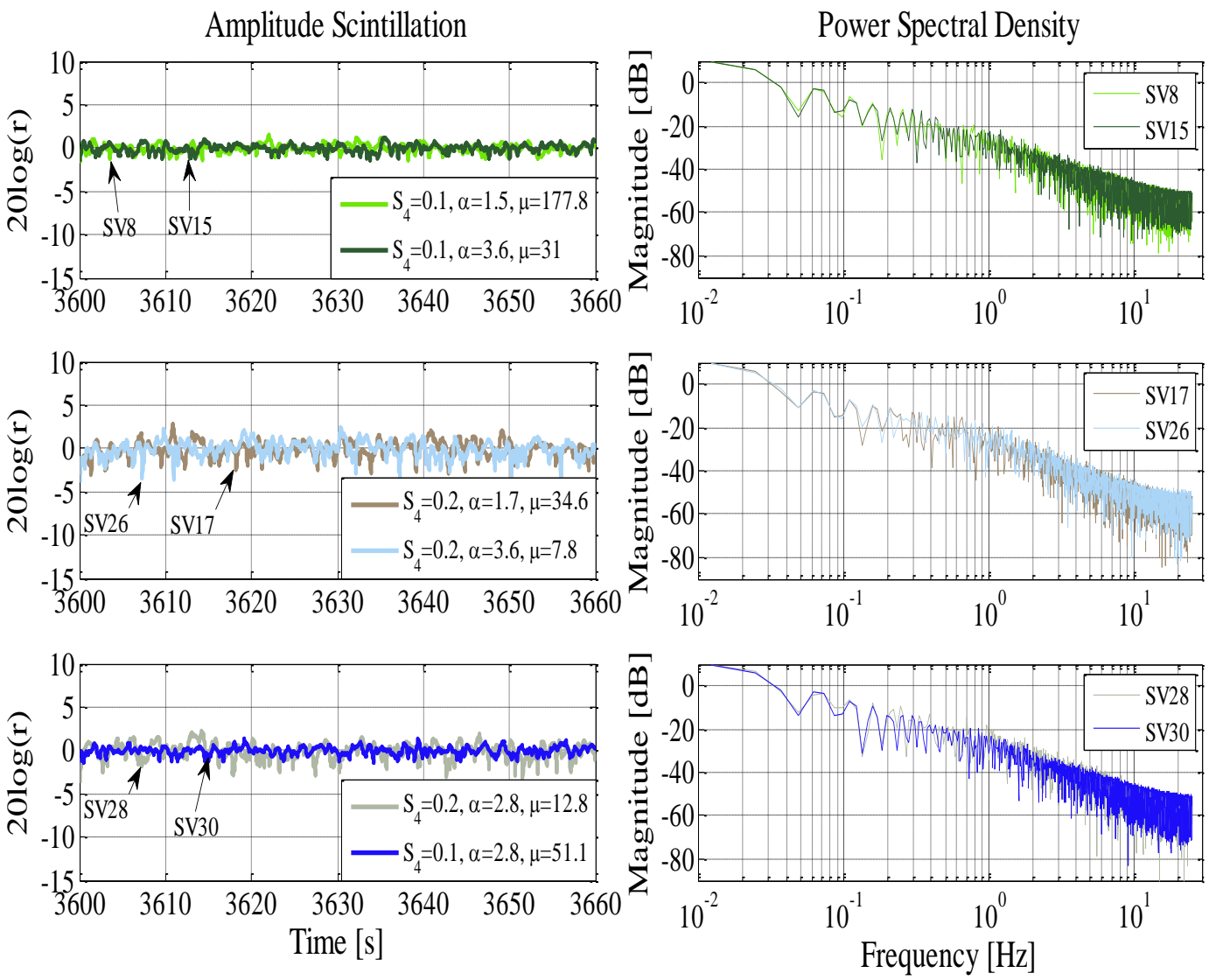

Figure 5.23: (Left column) one-min time series of amplitude scintillation simulated for the Rio de Janeiro station and satellites SV14, SV16, SV18, SV21, SV22, and SV27 during the time interval 01:00 UTC to 01:01 UTC on 22 July 2014, assuming the $\left(\mathrm{S}_{4}, \alpha, \mu\right)$ values displayed in the associated legends; (Right column) power spectral densities of the corresponding amplitude scintillation.

\subsubsection{Phase Scintillation $\phi_{I s c i n t(i, j)}(k)$}

In response to the $\mathrm{S}_{4}$ values observed at the Rio de Janeiro station during the time interval 01:00 UTC to 01:01 UTC on 20 December 2014 for transmissions from satellites SV14, SV16, SV18, SV21, SV22, and SV27 (0.5, 0.2, 0.3, 0.4, 0.3, and $\mathrm{S}_{4}=0.8$, respectively), the procedures described at the end of Subsection 5.1.6 yielded the following associated values of $\sigma_{\varphi}: 0.28 \mathrm{rad}, 0.08 \mathrm{rad}, 0.14 \mathrm{rad}, 0.19 \mathrm{rad}$, $0.15 \mathrm{rad}$, and $0.93 \mathrm{rad}$. The procedures were then applied to these $\sigma_{\varphi}$ values to generate 1-min time series of phase scintillation, as observed in Figure 5.24. Again, the statistical distribution of São José dos Campos station was used, because both stations are located in the same dip latitude range. 

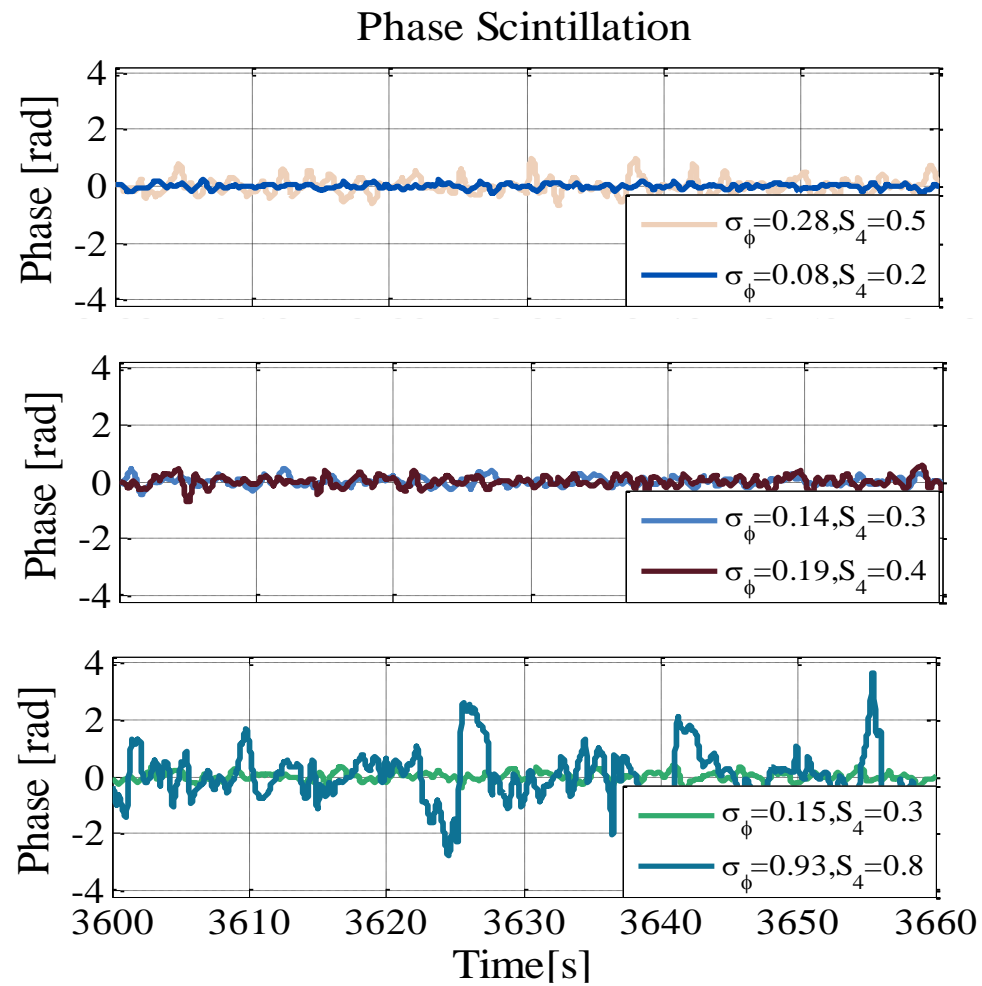

Figure 5.24: One-min time series of phase scintillation simulated for the Rio de Janeiro station and satellites SV14, SV16, SV18, SV21, SV22, and SV27 during the time interval 01:00 UTC to 01:01 UTC on 20 December 2014, assuming the $\left(\mathrm{S}_{4}, \sigma_{\varphi}\right)$ values displayed in the associated legends.

The phase deviation can reach 3.85 rad for SV27 in response to $\sigma_{\varphi}=0.93$ rad, as shown in the lower Panel of Figure 5.24. Large and rapid phase changes such as the simulated ones stress the receiver's phase lock loop and may result in cycle slips or loss of phase lock on the carrier phase signal. The simulated phase scintillation of the remaining satellites displays lower phase changes, due to small values of $\sigma_{\varphi}$.

\subsubsection{Time Series of the Pseudorange $P R_{(i, j)}(k)$}

The last Subsections presented individual results for all components of the pseudorange observable. The main objective of this section is to summarize and present results for the combined pseudorange model $P R_{(i, j)}(k)$ using all terms of equation (5.1). Figure 5.25 presents simulated pseudorange results for the Rio de Janeiro station and several visible satellites between 21:00 UTC on 19 December 2014 and 05:00 UTC on 20 December 2014. 


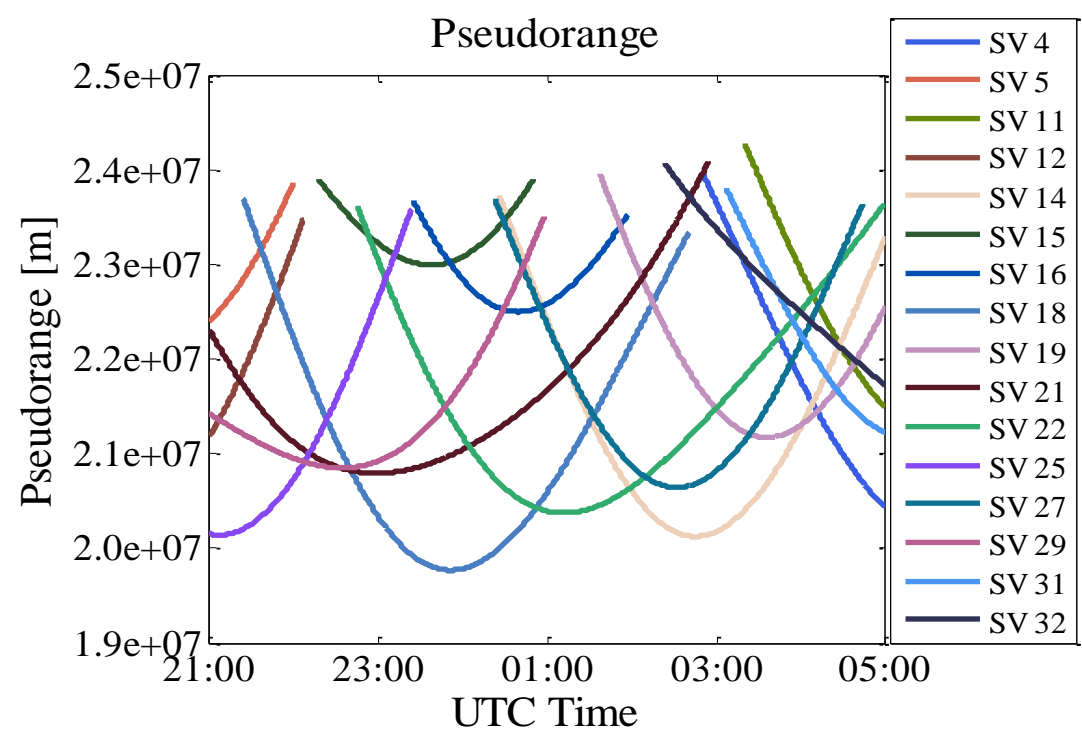

Figure 5.25: Time series for the pseudorange simulated for the Rio de Janeiro Station and several satellites on the night of 19-20 December 2014.

In Figure 5.25, it is possible to identify the magnitude of the pseudorange for

each visible GPS satellite and Rio de Janeiro station. However, these curves are dominated by the large contributions from the geometric distance and satellite clock error of each satellite-receiver pair. To highlight the contributions from the ionospheric and tropospheric delays, as well from the receiver clock, multipath, and random errors, only the combinations of their results are shown in Figure 5.26 for of each satellite-receiver pair.

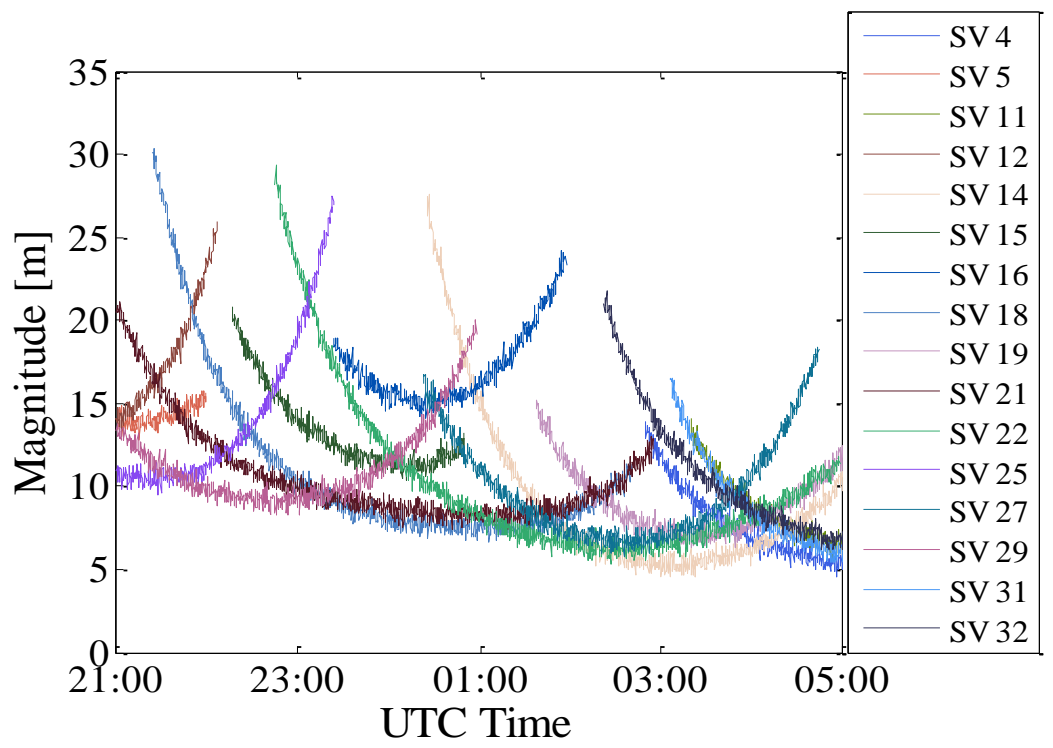

Figure 5.26: Time series of associated effects (ionospheric and tropospheric delays, receiver clock, multipath, and random errors) simulated for Rio de Janeiro station and different satellites on the night of 19-20 December 2014. 
The magnitude of the combined delays and errors present spatial and temporal variations as described in this Chapter. The results from the combined delays and errors are depicted for each satellite-receiver pair in Figure 5.26. For example, the Rio de Janeiro-SV14 and Rio de Janeiro-SV18 signals present combined delays equal to 4.5 meters around 03:23:00 UTC and 30.4 meters around 21:24:00 UTC, respectively. The combined delays for the other satellite-receiver pairs are limited by these minimum and maximum values. In addition to the effects from the status of the different media, the variations of the combined effects are also dependent on the elevation angle.

\subsubsection{Time Series of the Carrier Phase $\phi_{(i, j)}(k)$}

Similarly, the time series of carrier phase $\phi_{(i, j)}(k)$ were simulated using equation (5.2). Figure 5.27 presents simulated results from the carrier phase model for Rio de Janeiro station and different satellites between 21:00 UTC on 19 December 2014 and 05:00 UTC on 20 December 2014.

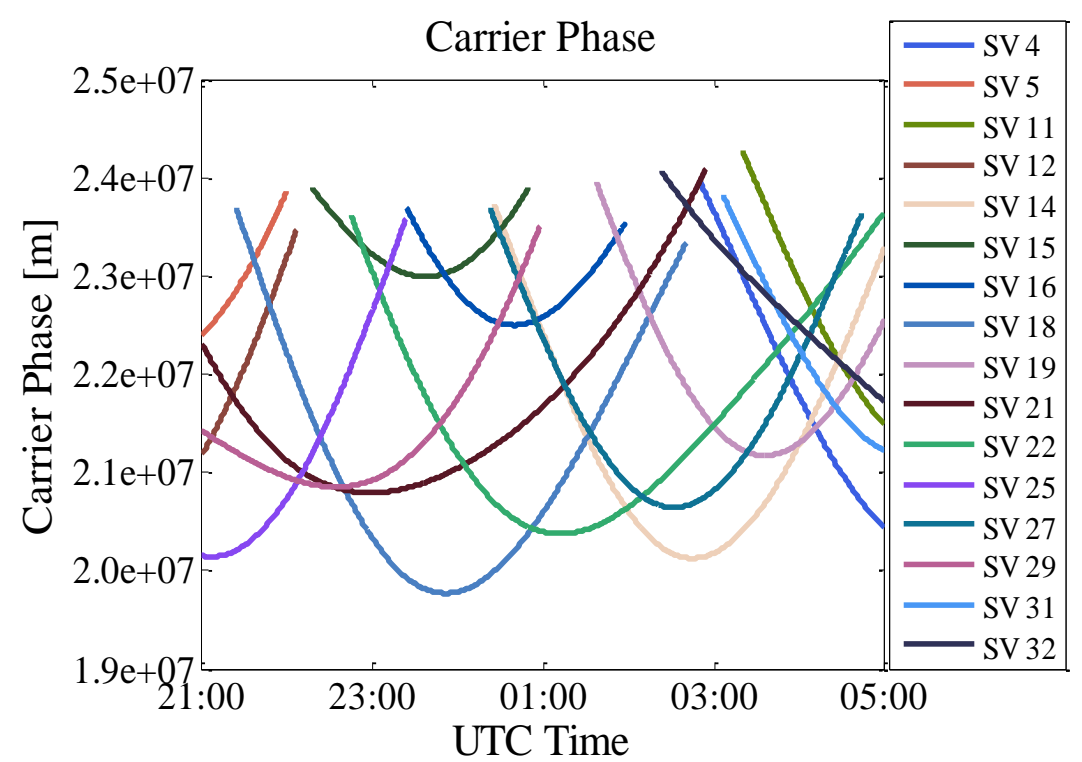

Figure 5.27: Time series of carrier phase simulated for the Rio de Janeiro station and several satellites on the night of 19-20 December 2014.

For the same reasons stated in the previous Subsection, the dominating contributions of the geometric distance and satellite clock errors will be subtracted from the results displayed in Figure 5.27. It is also important to remember that the 
carrier phase model includes the cycle ambiguity and phase scintillation. The results from the contributions of the remaining components after the subtraction is performed are shown in Figure 5.28.

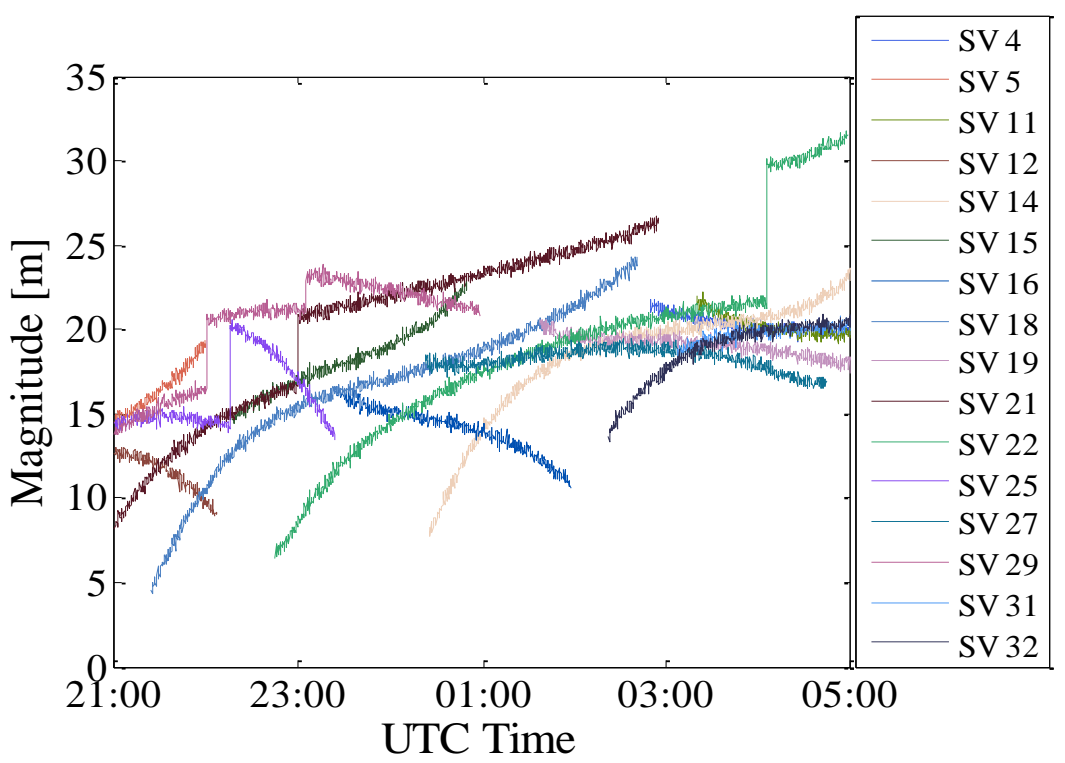

Figure 5.28: Time series of associated effects (ionospheric and tropospheric delays, cycle ambiguity, receiver clock, multipath, and random errors) simulated for Rio de Janeiro station and different satellites on the night of 19-20 December 2014.

As observed, the combined magnitude of the combined delays, ambiguity and errors, as described in this chapter for all effects, presents spatial and temporal variations. For example, the phase of the SV22 signal presents a combined delay equal to 31.7 meters around 05:00:00 UTC and that of the SV18 signal presents a combined delay equal to 4.6 meters around 21:24:00 UTC. Additionally, it is possible to identify simulated cycle slips in Figure 5.28. In this particular case study, SV21, SV22, SV25, and SV29 present simulated discontinuities in the interval between 4 to 8 meters, according the statistical distribution presented in Subsection 5.1.7. Again, in addition to the effects from the status of the different media, the variations of the combined effects are also dependent on the elevation angle.

The comparison between Figure 5.26 and Figure 5.28 can be summarized in the following: pseudorange observables are noisy but unambiguous and carrier phase measurements are smooth but subject to ambiguities. 


\subsubsection{Time Series of the Received Power $C_{(i, j)}(k)$}

Finally, this section presents 10-min time series of simulated received signal power for the Rio de Janeiro station and satellites SV14, SV16, SV18, SV21, SV22 and SV27 during the time interval 01:00 UTC to 01:10 UTC on the night of 20 December 2014, using equation (5.3), the system parameters described in Table 5.10 , and the associated empirical values of $\mathrm{S}_{4}$ (discussed in more detail in the next paragraph). The results are observed in the Panels of Figure 5.29, which clearly show that the probabilities of deep fades are very different for the six signals. Indeed, 10 realizations of the 10-min signals based on $\alpha-\mu$ distribution were performed. These results were also simulated using the statistical distribution of São José dos Campos, for the reason previously stated.
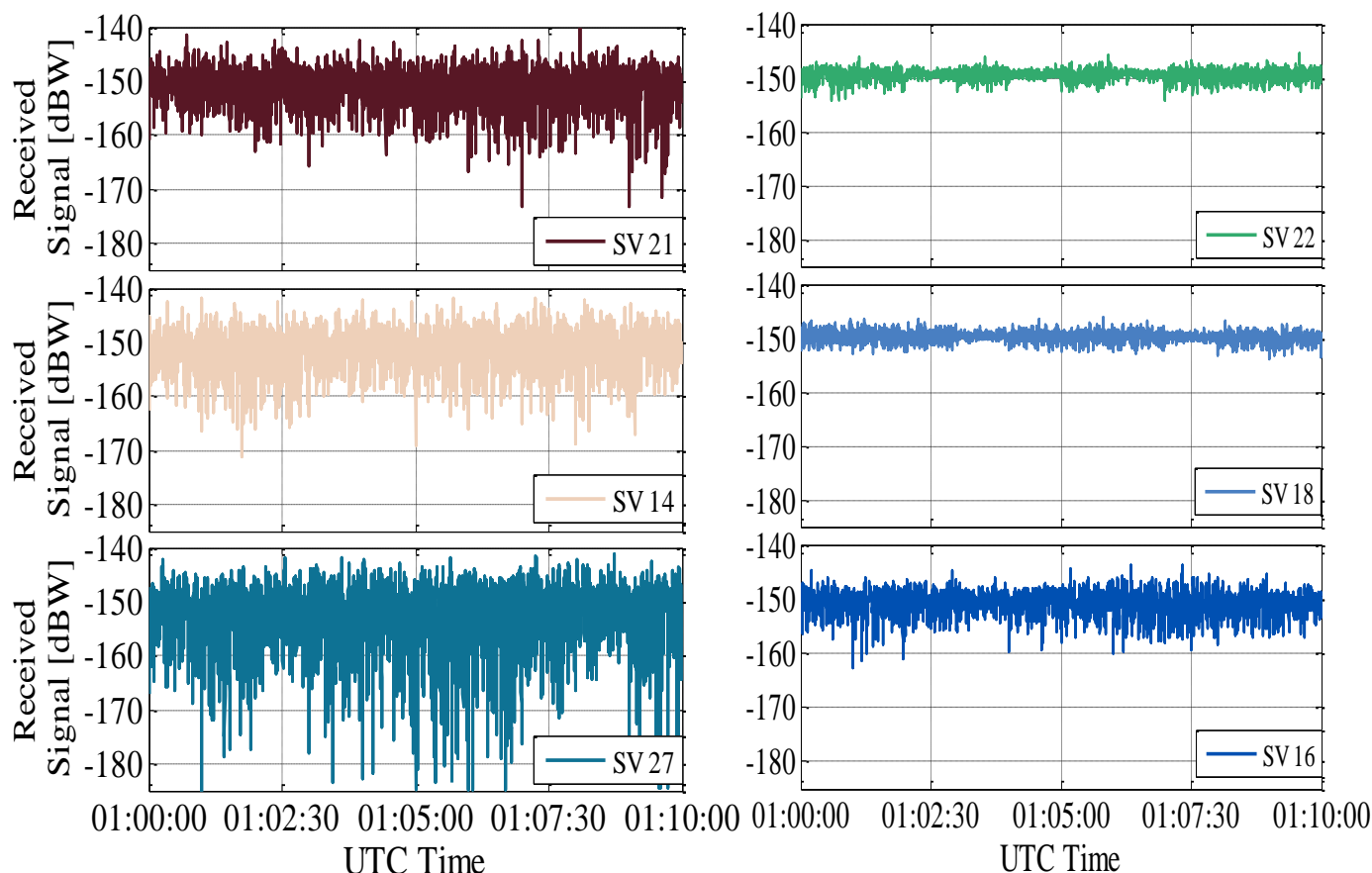
UTC Time

Figure 5.29: Time series of the received signal power for the Rio de Janeiro station and satellites SV14, SV16, SV18, SV21, SV22 and SV27 during the time interval 01:00 UTC to 01:10 UTC on the night of 20 December 2014.

The empirical values of $\mathrm{S}_{4}$ associated with the associated time interval for each satellite vary in the intervals: SV14 $(0.6,0.7)$; SV16 $(0.4,0.5)$; SV18 $(0.1,0.3)$; SV21 $(0.6,0.7)$; SV22 $(0.1,0.3)$; and SV27 $(0.8,0.9)$. Considering the relation between $\alpha-\mu$ coefficients and the fundamental role of the index $S_{4}$, it is possible to 
note that the minimum received signal powers for the satellite-receiver pairs are -171.2 dBW, -161.2 dBW, -153.7 dBW, -173.1 dBW, -153.9 dBW and -184.7.0 $\mathrm{dBW}$, respectively. Note that one objective is to simulate the signal in space for the worse case in order to evaluate the GBAS system.

The minimum received signal for GPS L1 is $-164.5 \mathrm{dBW}$ and $-158.5 \mathrm{dBW}$ for P and C/A code, respectively (SPS, 2018), if one satellite presents a received signal power below to the threshold, the corresponding channel is not available. A fading occurrence is defined when the received power decrease below the minimum operational value of the receiver. In this event, the low received signal power condition stresses the receiver, causing loss of lock.

\subsection{Definition of Active Channels Based on the CALIBRA/CIGALA Network}

The main objective of this section is to discuss the probability distribution of occurrences of the number of active and visible satellites that transmit GPS L1 signals and are received in different Brazilian stations, as well as the associated values of $\mathrm{S}_{4}$ indices. The data analyzed in this section were acquired by four CIGALA/CALIBRA stations located at different geomagnetic latitudes during the time interval: 01 January 2012 to 31 December 2016. The $S_{4}$ indices were estimated at every 60 seconds. Table 5.11 shows the name and location of the stations that contributed with data. To position these stations with respect to the EIA, where the most intense scintillation events are observed, it should be remembered that its southern crest is generally located within the geomagnetic latitude interval $\left(-15^{\circ}\right.$, $20^{\circ}$ ). Thus, Figure 5.30 shows that Palmas (PALM) is the station closest to the geomagnetic equator, where EPBs are generated. Presidente Prudente (PRU1 and PRU2 station) and São José dos Campos (SJCI, SJCE and SJCU station), on the other hand, are located near the southern crest of EIA. Finally, Porto Alegre (POAL station) is in opposite side of the EIA crest, away from the geomagnetic equator. 
Table 5.11: Description of the CIGALA/CALIBRA receiver stations.

\begin{tabular}{|c|l|c|c|c|}
\hline $\begin{array}{c}\text { Receiver } \\
\text { Station }\end{array}$ & \multicolumn{1}{|c|}{ City } & $\begin{array}{c}\text { Latitude } \\
\mathbf{(}^{\circ}\end{array}$ & $\begin{array}{c}\text { Longitude } \\
\mathbf{(}^{\circ} \mathbf{)}\end{array}$ & $\begin{array}{c}\text { Dip latitude } \\
\mathbf{(}^{\circ}\end{array}$ \\
\hline PALM & Palmas & -10.1996 & -48.3113 & -7.7832 \\
\hline PRU1 & Presidente Prudente & -22.1200 & -51.4087 & -15.8552 \\
\hline PRU2 & Presidente Prudente & -22.1220 & -51.4071 & -15.8576 \\
\hline SJCI & São José dos Campos & -23.2076 & -45.8596 & -19.8248 \\
\hline SJCE & São José dos Campos & -23.2075 & -45.8597 & -19.8247 \\
\hline SJCU & São José dos Campos & -23.2106 & -45.9566 & -19.7695 \\
\hline POAL & Porto Alegre & -30.0739 & -51.1197 & -22.0104 \\
\hline
\end{tabular}

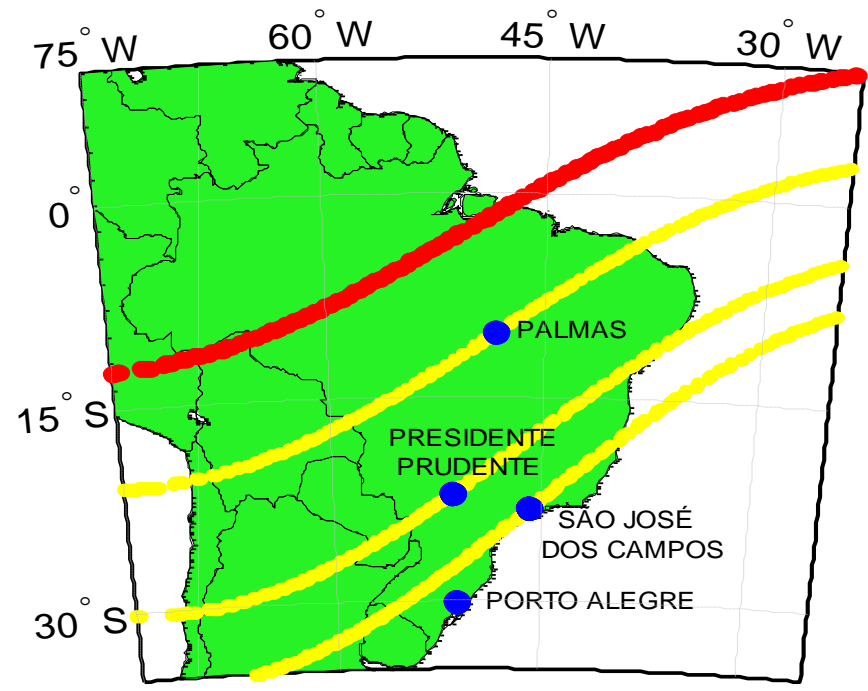

Figure 5.30: Positions of Palmas, Presidente Prudente, São José dos Campos and Porto Alegre receiver stations, with respect to the geomagnetic equator and the southern crest of the EIA.

This analysis considers different combinations of ranges of the following parameters: (i) EPB season in the Brazilian sector, closely associated with that of ionospheric scintillation; (ii) solar activity, represented by the F10.7 index.

Table 5.12: Season and solar activity parameters.

\begin{tabular}{|c|c|c|}
\hline $\begin{array}{l}\text { April-Aug }- \text { g } \\
\text { September }\end{array}$ & $\begin{array}{l}\text { ust } \\
\text { - March }\end{array}$ & $\begin{array}{l}\text { AA } \\
\text { SM }\end{array}$ \\
\hline Solar activity (F10.7 index): & $\begin{array}{l}\mathrm{F} 10.7<100 \\
100 \leq \mathrm{F} 10.7<150 \\
\mathrm{~F} 10.7 \geq 150\end{array}$ & $\begin{array}{l}\mathrm{LO} \\
\mathrm{MD} \\
\mathrm{HG}\end{array}$ \\
\hline
\end{tabular}


Figure 5.31 to Figure 5.34 show empirical distribution of occurrence for visible (above the $20^{\circ}$ elevation mask) and active GPS satellites tracked by Palmas, Presidente Prudente, São José dos Campos and Porto Alegre stations, considering the different combinations of season and solar activity parameters described in Table 5.12, using different color bars between 01 January 2012 to 31 December 2016.

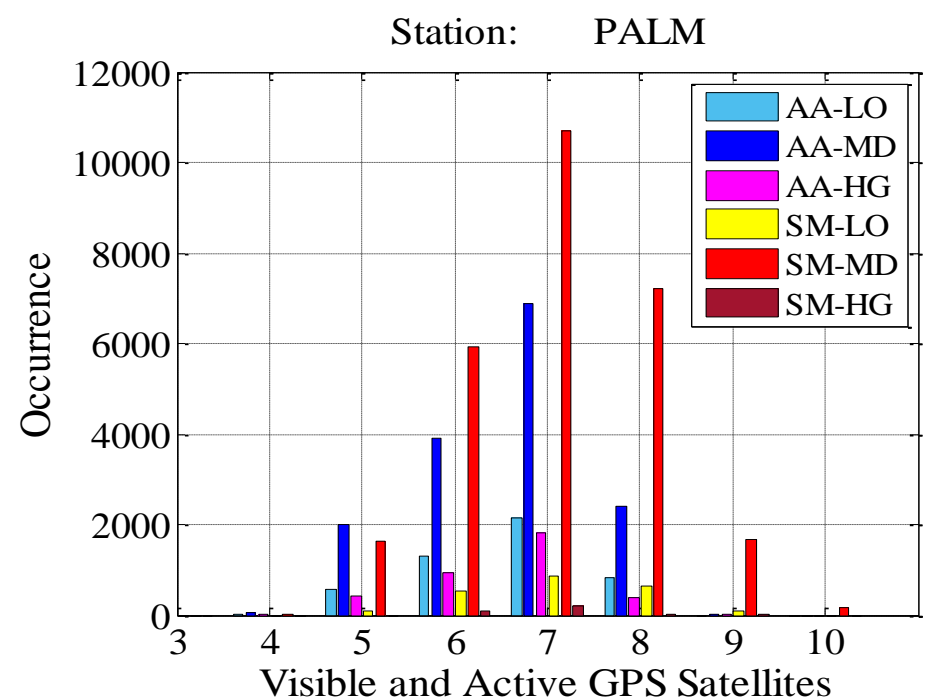

Figure 5.31: Empirical distributions of occurrences of visible and active GPS satellites tracked by the PALM station (Palmas, dip latitude: $-7.78^{\circ}$ ) for different combinations of seasons and solar activities.

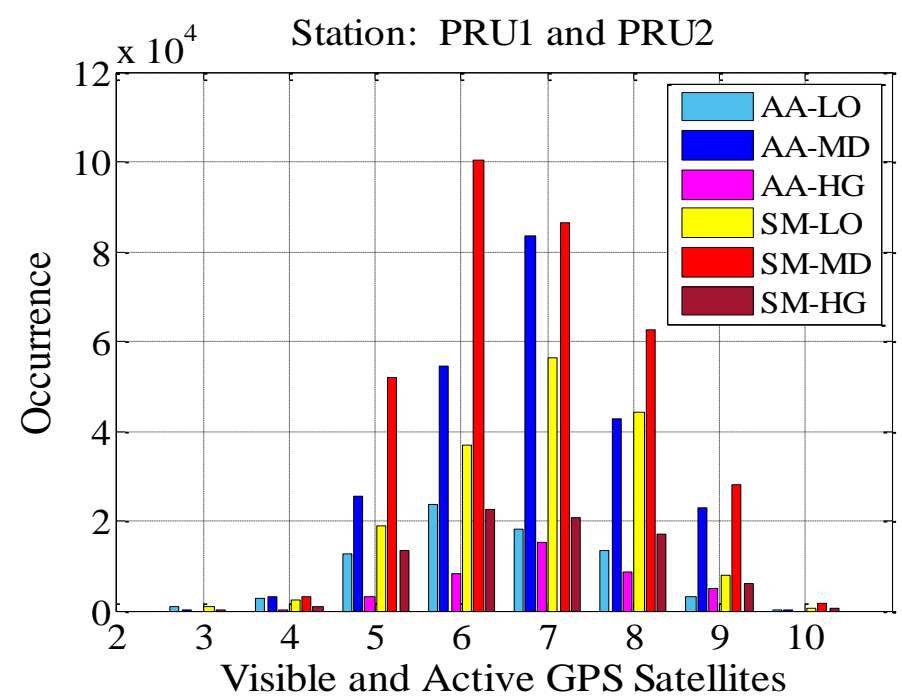

Figure 5.32: Empirical distributions of occurrences of visible and active GPS satellites tracked by the PRU1 and PRU2 stations (Presidente Prudente, dip latitude: $-15.86^{\circ}$ ) for different combinations of seasons and solar activities. 


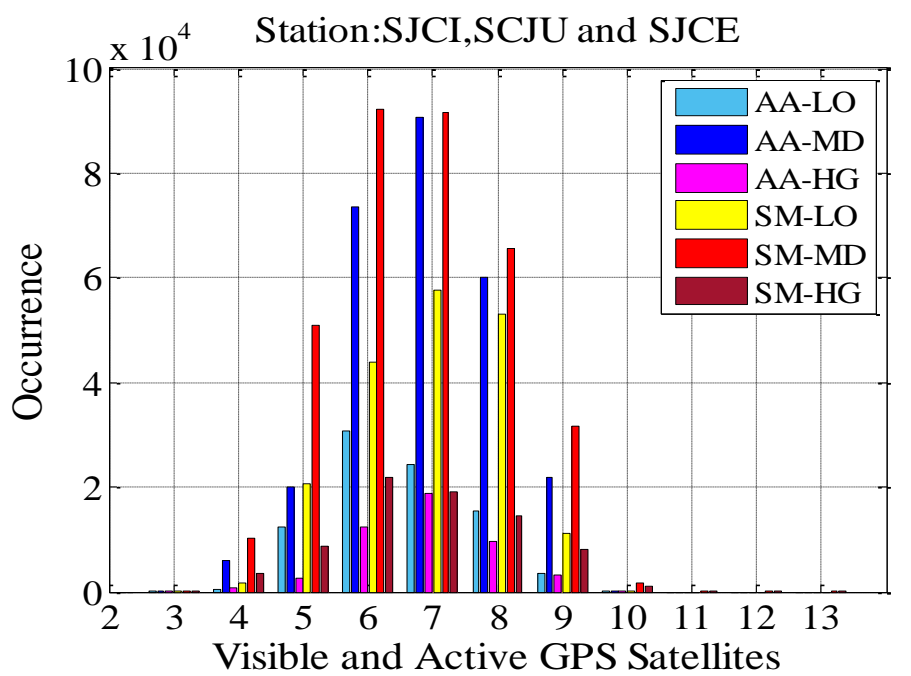

Figure 5.33: Empirical distributions of occurrences of visible and active GPS satellites tracked by the SJCI, SCJU and SJCE stations (São José dos Campos, dip latitude: $-19.82^{\circ}$ ) for different combinations of seasons and solar activities.

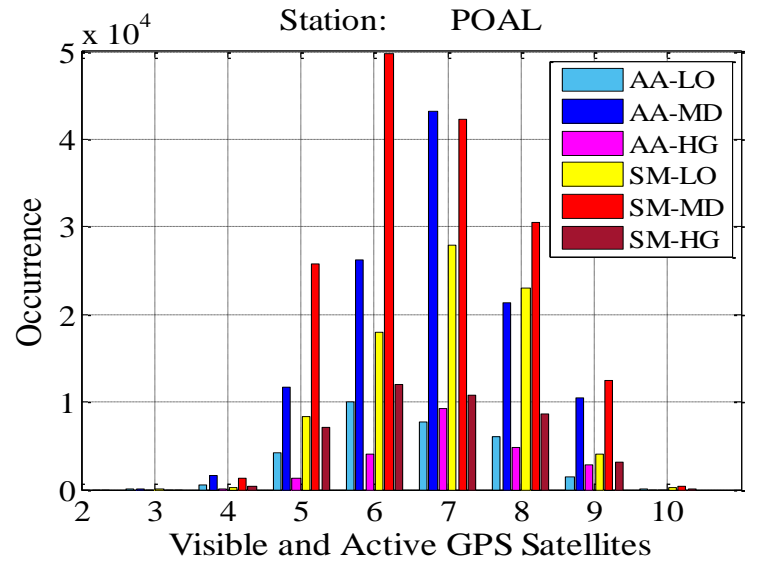

Figure 5.34: Empirical distributions of occurrences of visible and active GPS satellites tracked by the POAL station (Porto Alegre, dip latitude: $-22.01^{\circ}$ ) for different combinations of seasons and solar activities.

The probabilities density functions based on the occurrences displayed in Figures 5.31 to 5.34 are estimated for each station, season and solar activity parameters, and can be seen in Table 5.13. In combination with Figures 5.31 to 5.34, this Table shows that the majority of occurrences are associated with 6 or 7 tracked satellites from September to March, in the presence of medium solar activity $(100 \leq \mathrm{F} 10.7<150)$ for all receiver locations. In addition, the minimum of 3 tracked satellites is observed for the combinations of season and solar activity ranges for most stations. The exception is the PALM station, which tracked the 
minimum of 4 satellites. On the other hand, the maximum number of visible and active GPS satellites tracked by Palmas, Presidente Prudente and Porto Alegre stations are 10 satellites. It should be noted that the São José dos Campos stations also tracked from 11 to 13 satellites, but in a very small number of cases.

Table 5.13: Probabilities of the number of visible GPS satellites for each of the four receiver stations of the CIGALA/CALIBRA network.

\begin{tabular}{|c|c|c|c|c|c|c|c|c|c|c|c|}
\hline \multicolumn{12}{|c|}{ Number of visible GPS satellites } \\
\hline & 3 & 4 & 5 & 6 & 7 & 8 & 9 & 10 & 11 & 12 & 13 \\
\hline \multicolumn{12}{|c|}{ Palmas (PALM) dip latitude: $\mathbf{- 7 . 7 8 ^ { \circ }}$} \\
\hline AA-LO (\%) & - & 0.14 & 12.07 & 26.57 & 44.16 & 17.05 & - & - & - & - & - \\
\hline AA-MD (\%) & - & 0.34 & 12.97 & 25.70 & 45.10 & 15.79 & 0.10 & - & - & - & - \\
\hline AA-HG (\%) & - & 0.96 & 11.71 & 26.20 & 49.96 & 10.92 & 0.25 & - & - & - & - \\
\hline SM-LO (\%) & - & - & 4.70 & 23.80 & 38.30 & 29.17 & 4.03 & - & - & - & - \\
\hline SM-MD (\%) & - & 0.10 & 5.93 & 21.65 & 39.22 & 26.39 & 6.17 & 0.54 & - & - & - \\
\hline SM-HG (\%) & - & - & - & 23.36 & 61.68 & 10.28 & 4.67 & - & - & - & - \\
\hline \multicolumn{12}{|c|}{ Presidente Prudente (PRU1 and PRU2) } \\
\hline AA-LO (\%) & 1.21 & 3.58 & 16.92 & 31.72 & 24.37 & 17.78 & 4.40 & - & - & - & - \\
\hline AA-MD (\%) & 0.08 & 1.32 & 10.99 & 23.49 & 35.85 & 18.37 & 9.87 & 0.02 & - & - & - \\
\hline AA-HG (\%) & - & 0.24 & 7.36 & 20.37 & 38.17 & 21.70 & 12.17 & - & - & - & - \\
\hline SM-LO (\%) & 0.65 & 1.34 & 11.27 & 21.93 & 33.54 & 26.24 & 4.73 & 0.29 & - & - & - \\
\hline SM-MD (\%) & 0.01 & 0.92 & 15.58 & 29.98 & 25.82 & 18.78 & 8.45 & 0.45 & - & - & - \\
\hline SM-HG (\%) & - & 1.15 & 16.59 & 27.86 & 25.62 & 20.77 & 7.50 & 0.51 & - & - & - \\
\hline \multicolumn{12}{|c|}{ São José dos Campos (SJCI, SCJU and SJCE) dip latitude: $\mathbf{- 1 9 . 8 2 ^ { \circ }}$} \\
\hline AA-LO (\%) & 0.01 & 0.56 & 14.28 & 35.47 & 27.98 & 17.79 & 3.92 & - & - & - & - \\
\hline AA-MD (\%) & - & 2.17 & 7.35 & 26.99 & 33.32 & 22.13 & 7.99 & 0.05 & - & - & - \\
\hline AA-HG (\%) & 0.03 & 1.76 & 5.51 & 26.22 & 39.32 & 20.32 & 6.82 & 0.01 & - & - & - \\
\hline SM-LO (\%) & 0.05 & 0.93 & 10.91 & 23.25 & 30.65 & 28.23 & 5.91 & 0.08 & - & - & - \\
\hline SM-MD (\%) & 0.04 & 2.97 & 14.76 & 26.79 & 26.59 & 19.06 & 9.19 & 0.48 & 0.05 & 0.05 & 0.01 \\
\hline SM-HG (\%) & 0.11 & 4.38 & 11.21 & 28.10 & 24.81 & 18.84 & 10.39 & 1.43 & 0.37 & 0.30 & 0.05 \\
\hline \multicolumn{12}{|c|}{ Porto Alegre (POAL) dip latitude: $-22.01^{\circ}$} \\
\hline AA-LO (\%) & 0.24 & 1.72 & 14.23 & 33.38 & 25.76 & 20.03 & 4.63 & 0.01 & - & - & - \\
\hline $\mathrm{AA}-\mathrm{MD}(\%)$ & 0.01 & 1.34 & 10.22 & 22.94 & 37.72 & 18.62 & 9.16 & - & - & - & - \\
\hline AA-HG $(\%)$ & - & 0.23 & 5.63 & 18.10 & 41.81 & 21.78 & 12.46 & - & - & - & - \\
\hline SM-LO (\%) & 0.02 & 0.31 & 10.18 & 22.01 & 34.16 & 28.10 & 4.99 & 0.23 & - & - & - \\
\hline SM-MD (\%) & - & 0.81 & 15.87 & 30.62 & 26.03 & 18.77 & 7.68 & 0.23 & - & - & - \\
\hline SM-HG (\%) & - & 1.05 & 16.90 & 28.37 & 25.46 & 20.48 & 7.40 & 0.35 & - & - & - \\
\hline
\end{tabular}


For the sake of completeness, the cumulative distribution of the number of visible and active GPS satellites tracked by the four receiver stations of the CIGALA/CALIBRA network described in Table 5.11, according to season and solar activity ranges, are shown in Figures 5.35 to 5.38.

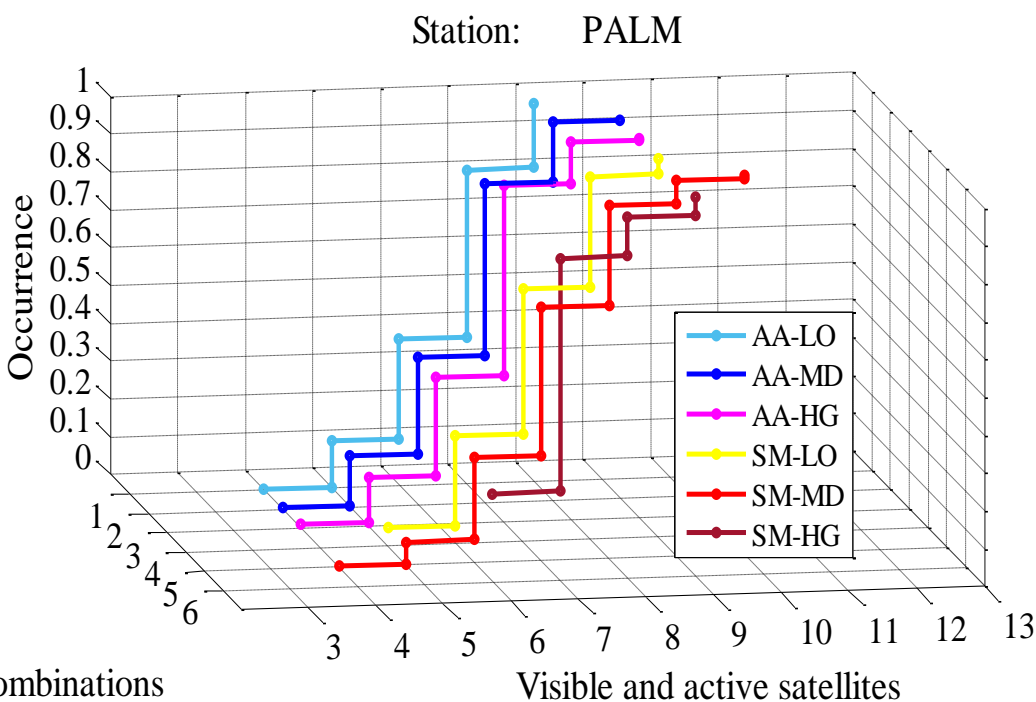

Figure 5.35: Empirical cumulative distribution of the number of visible and active GPS satellites tracked by the PALM station (Palmas, dip latitude: $-7.78^{\circ}$ ), according to combinations of season and solar activity ranges.

Station: PRU1 and PRU2

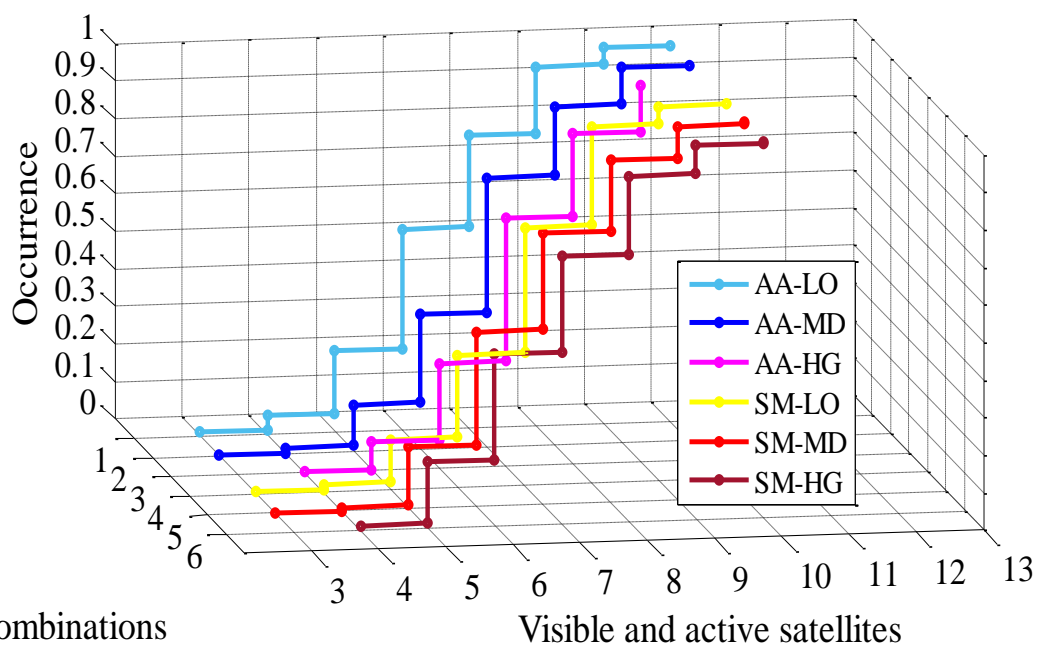

Figure 5.36: Empirical cumulative distribution of the number of visible and active GPS satellites tracked by the PRU1 and PRU2 station (Presidente Prudente, dip latitude: $-15.82^{\circ}$ ), according to combinations of season and solar activity ranges. 


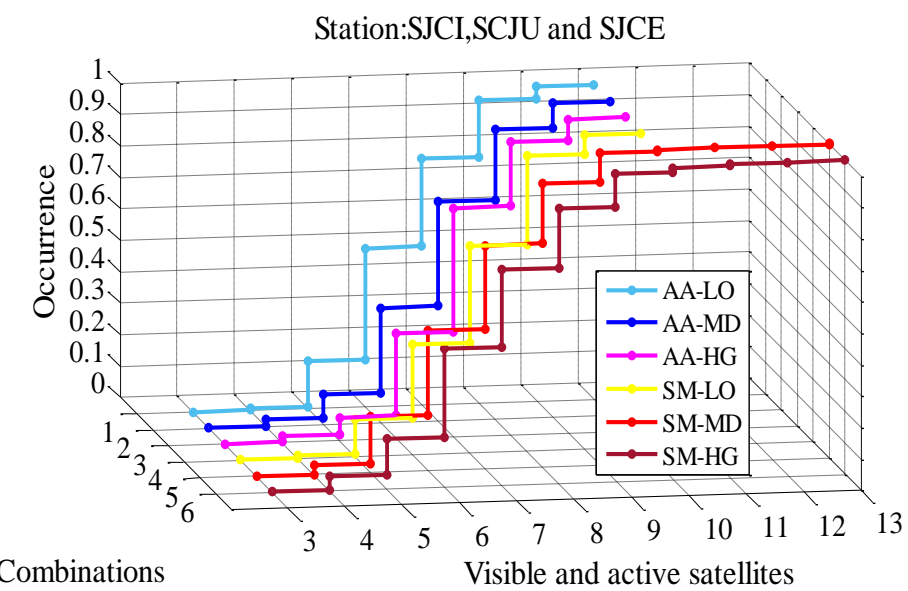

Figure 5.37: Empirical cumulative distribution of the number of visible and active GPS satellites tracked by the SJCI, SCJU and SJCE station (São José dos Campos, Dip latitude: $-19.82^{\circ}$ ) according to combinations of season and solar activity ranges.

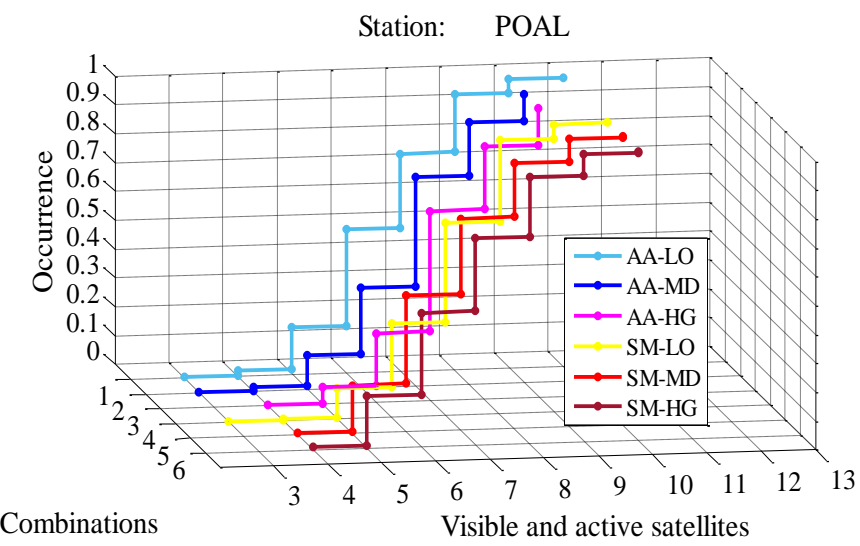

Figure 5.38: Empirical cumulative distribution of the number of visible and active GPS satellites tracked by the POAL station (Porto Alegre, Dip latitude: $-22.01^{\circ}$ ), according to combinations of season and solar activity ranges.

\section{Combinations of numbers of GPS satellites according $S_{4}$ intervals}

This section presents statistical distributions of combinations of $\mathrm{S}_{4}$ index intervals in the active satellite-receiver pairs, simultaneously observed at each time instant. For the present thesis, the experimental data used to estimate the $\mathrm{S}_{4}$ index were recorded by the receiver stations listed in Table 5.11, selected by their dip latitudes. The data were measured by Septentrio PolaRxS scintillation monitors with ultralow noise oven-controlled crystal oscillators for GPS L1 (1575.42 MHz) signals at the sampling frequency of $50 \mathrm{~Hz}$. For each satellite-receiver pair, sixty seconds of data (corresponding to 3000 samples) are used to estimate the associated 
$\mathrm{S}_{4}$ value. Thus, consecutive data records are spaced by $1 \mathrm{~min}$. Each data record available for the present analysis, corresponding to a single time instant, lists ( $\mathrm{S}_{4 \mathrm{n}}$, $\left.\mathrm{SV}_{\mathrm{n}}\right), \mathrm{n}=1, \ldots, \mathrm{N}$, where $\mathrm{N}$ is the number of active satellite-receiver pairs at that time instant.

The $\mathrm{S}_{4}$-index intervals adopted for the present analysis are summarized in Table 5.14. Each of the integers $\mathrm{m}, \mathrm{n}, \mathrm{p}, \mathrm{q}, \mathrm{r}$ indicates the number of satellitereceiver pairs with $S_{4}$ values in the associated interval, represented in the Table element immediately above, at each time instant. Naturally, $m+n+p+q+r$ is equal to $\mathrm{N}$, for the corresponding time instant. For example, the combination 20310 indicates that, at a particular time instant, six satellite were active and visible, two satellite-receiver pairs experienced $S_{4} \leq 0.3$, three experienced $0.5<S_{4} \leq 0.7$, and one experienced $0.7<\mathrm{S}_{4} \leq 0.9$.

Table 5.14 Amplitude scintillation intervals and their representations.

\begin{tabular}{|c|c|c|c|c|c|}
\hline $\mathrm{S}_{4}$ Interval & $\mathrm{S}_{4} \leq 0.3$ & $0.3<\mathrm{S}_{4} \leq 0.5$ & $0.5<\mathrm{S}_{4} \leq 0.7$ & $0.7<\mathrm{S}_{4} \leq 0.9$ & $\mathrm{~S}_{4}>0.9$ \\
\hline $\begin{array}{c}\text { Represen- } \\
\text { tation }\end{array}$ & $\mathrm{m} 0000$ & $0 \mathrm{n} 000$ & $00 \mathrm{p} 00$ & $000 \mathrm{q} 0$ & $0000 \mathrm{r}$ \\
\hline
\end{tabular}

As an example of the results from the current processing, the Panels of Figure 5.39 present the occurrences (numbers of cases) of mnpqr, for the combination of $\mathrm{N}=6$ active satellite-receiver pairs, season and solar activity, estimated from the three São José dos Campos stations between 01 January 2012 to 31 December 2016. It should be remembered that these stations presented between 3 to 13 active and visible satellites, according to Table 5.13.

The upper Panel of Figure 5.39 indicates that the histograms associated with the combinations AA-LO, AA-MD, and AA-HG yield classes (60000, 51000, 50100, 50001, and 42000) dominated by weak to moderate scintillation in all satellite-receiver pairs, with initially high numbers of cases that decrease very fast. It is evident that the classes associated with the above combinations are not present in most of the upper Panel, as well as in the center and lower Panels of Figure 5.39, that display occurrences of strong scintillation. 

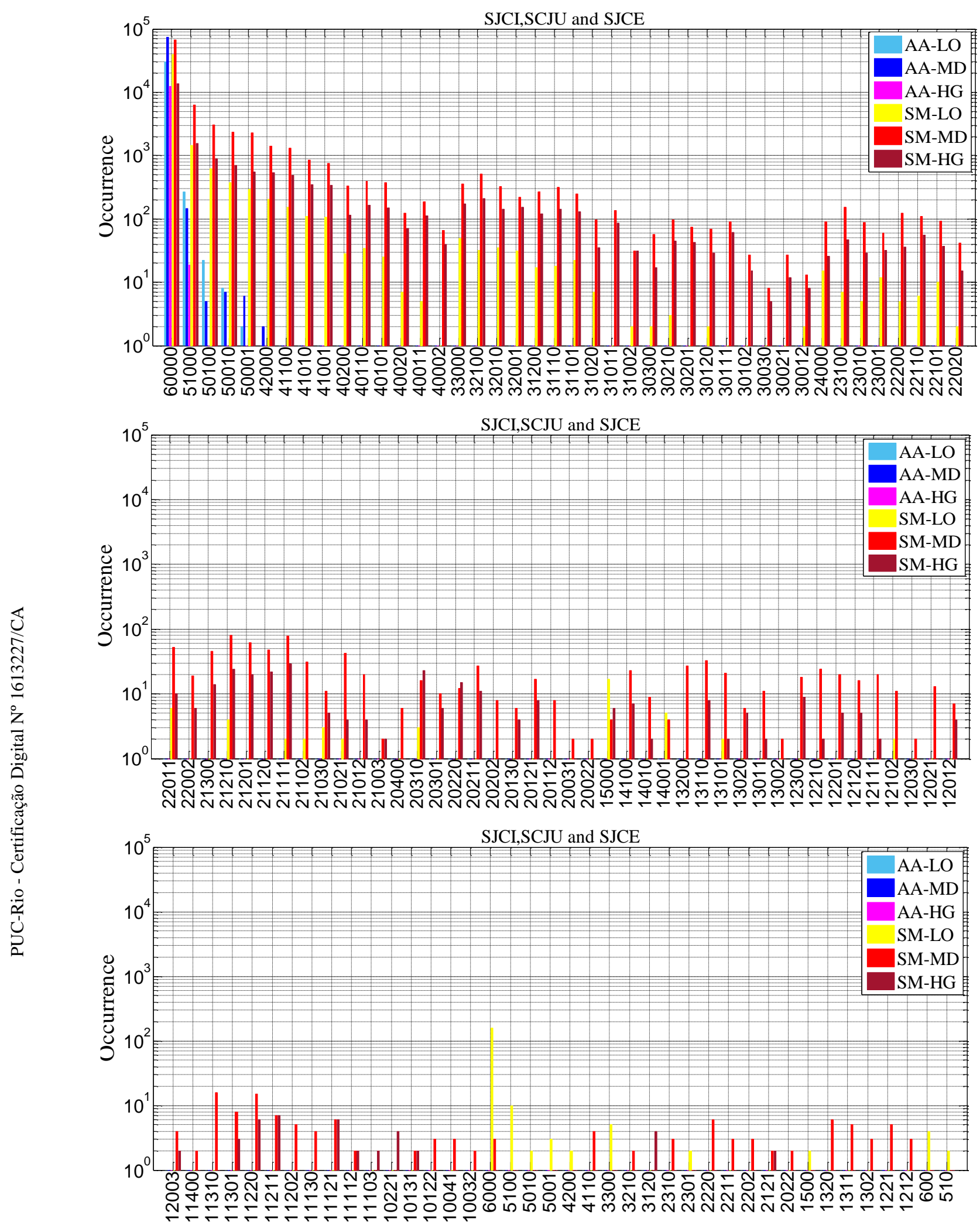

Figure 5.39: Empirical distribution of classes of satellite-receiver pairs with different intervals of $\mathrm{S}_{4}$ values, for different combinations of season and solar activity, considering 6 visible and active satellites, based on measurements from the three stations located in São José dos Campos (Top, middle and bottom panels). 
It should be remembered that September to March (SM) is the season of EPB and scintillation occurrence in the Brazilian region (Moraes et al., 2017). The upper Panel of Figure 5.39 additionally indicates that the histograms associated with the combinations SM-LO, SM-MD, and SM-HG also yield classes (60000, 51000, 50100, 50001, and 42000) dominated by weak to moderate scintillation in all satellite-receiver pairs, with initially high numbers of cases. In opposition to the observations in the previous paragraph, the same Panel clearly shows the significant occurrence of classes associated with moderate to strong values of $S_{4}\left(0.5<S_{4} \leq\right.$ $0.7,0.7<S_{4} \leq 0.9$ and $\left.S_{4}>0.9\right)$ in several satellite-receiver pairs. Actually, occurrences of classes associated with moderate to strong values of $\mathrm{S}_{4}$ extend to the center and lower Panels of Figure 5.39. However, the numbers of cases associated with these classes are relatively small. Indeed, there are more than $10^{4}$ cases of class 60000 for the combinations SM-LO, SM-MD, and SM-HG. On the other hand, most of the classes in the center and lower Panels are associated with occurrences less than $10^{2}$ and $10^{1}$, respectively. 


\section{GROUND BASED AUGMENTATION SYSTEM - SIMULATION MODEL}

A Ground Based Augmentation Systems (GBAS) is designed to provide enhanced levels of service over that of the primary GNSS constellation, in such a way as to support all phases of airport runaway approaches, being responsible for generating corrections and creating alerts over protection levels. In this context, a GBAS must additionally apply a comprehensive set of monitoring algorithms to detect possible failures in the GPS signals and to isolate detected failures. An Integrity Monitor Testbed (IMT) of the GBAS Ground Facility has been developed to evaluate whether a GBAS can meet a list of requirements (Skidmore and the LAAS KTA Group, 1998; Pullen, 2011; Xie, 2004; Lee, 2005). Figure 6.1 shows the functional blocks of the GBAS Ground Facility coded and all its functions will be described in this chapter. The description closely follows the ones by Skidmore and the LAAS KTA Group (1998), as well as by Xie (2004) and Lee (2005), with the main purpose of simulating the effects from the equatorial and low-latitude ionosphere (and secondarily, from the troposphere and multipath) on the performance of a GBAS. Note that each of its blocks is indicated by an alphabetical symbol and their interconnections by numbers described in Table 6.1.

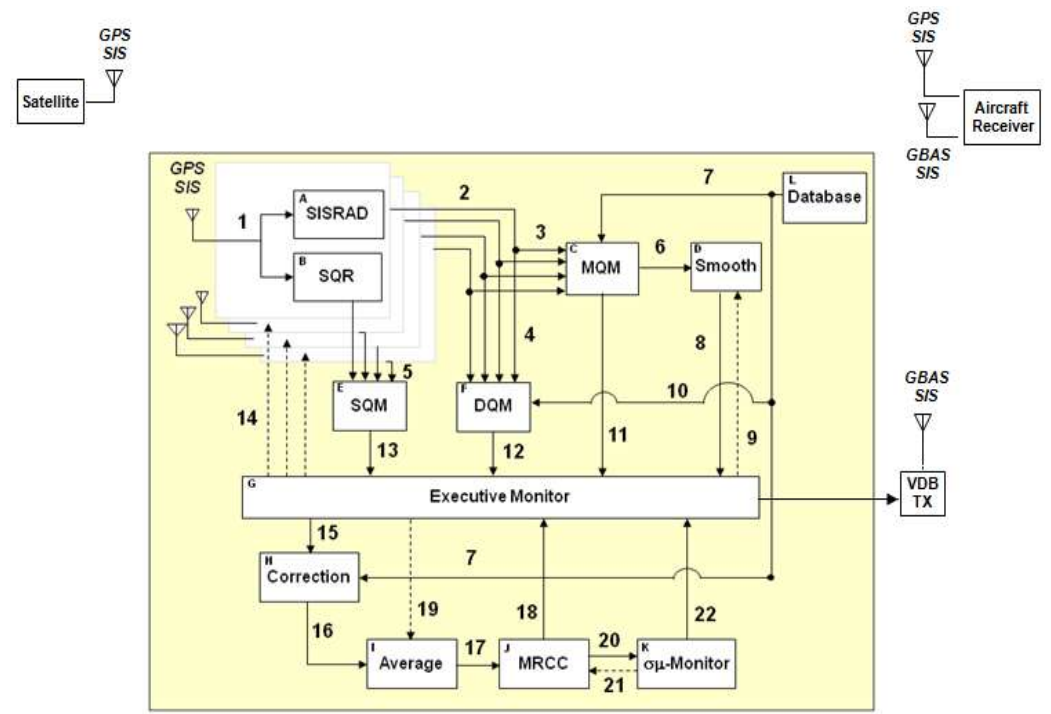

Figure 6.1: Block Diagram of the GBAS Ground Facility model.

[Adapted from Skidmore and the LAAS KTA Group (1998)]. 
Table 6.1: GBAS data interfaces.

\begin{tabular}{|c|c|}
\hline Interface & Data \\
\hline 1 & $\begin{array}{l}\text { Pseudorange } P R_{(i, j)}(k) \text {, carrier phase } \phi_{(i, j)}(k) \text {, received signal } \\
C_{(i, j)}(k) \text { and navigation data }\end{array}$ \\
\hline 2 & $\begin{array}{l}\text { Pseudorange } P R_{(i, j)}(k) \text { and carrier phase } \phi_{(i, j)}(k) \text {, ECEF satellite } \\
\text { coordinates } x_{i}(k), y_{i}(k), z_{i}(k) \text { and elevation angle } \varepsilon_{(i, j)}(k)\end{array}$ \\
\hline 3 & Pseudorange $P R_{(i, j)}(k)$ and carrier phase $\phi_{(i, j)}(k)$ \\
\hline 4 & ECEF satellite coordinates $x_{i}(k), y_{i}(k), z_{i}(k)$ and navigation data \\
\hline 5 & Pseudorange $P R_{(i, j)}(k)$, carrier phase $\phi_{(i, j)}(k)$ and $C / N_{o(i, j)}(k)$ \\
\hline 6 & Pseudorange $P R_{(i, j)}(k)$ and carrier phase $\phi_{(i, j)}(k)$ \\
\hline 7 & Reference antenna locations $x_{j}(k), y_{j}(k)$ and $z_{j}(k)$ \\
\hline 8 & Smoothed pseudorange $P R_{s m(i, j)}(k)$ and carrier phase $\phi_{(i, j)}(k)$ \\
\hline 9 & Filter reset command \\
\hline 10 & ECEF satellite coordinates $x_{i(Y e)}(k), y_{i(Y e)}(k), z_{i(Y e)}(k)$ \\
\hline 11 & Measurement Quality Monitor flags and Reference antenna locations \\
\hline 12 & $\begin{array}{l}\text { Data Quality Monitor flags and ECEF satellite coordinates } x_{i}(k) \text {, } \\
y_{i}(k), z_{i}(k)\end{array}$ \\
\hline 13 & Signal Quality Monitor flags \\
\hline 14 & Receiver reset flags \\
\hline 15 & Smoothed pseudorange $P R_{s m(i, j)}(k)$ and carrier phase $\phi_{(i, j)}(k)$ \\
\hline 16 & $\begin{array}{l}\text { Smoothed pseudorange corrections } P R_{s m c(n, m)}(k) \text { and carrier phase } \\
\text { corrections } \phi_{c(n, m)}(k)\end{array}$ \\
\hline 17 & $\begin{array}{l}\text { Pseudorange smoothed clock adjustment } P R_{s c a(n, m)}(k) \text {, the carrier } \\
\text { phase clock adjustment } \phi_{c a(n, m)}(k) \text {, the pseudorange corrections } \\
P R_{\operatorname{corr}(n)}(k) \text { and carrier phase corrections } \phi_{\operatorname{corr}(n)}(k) \text {. }\end{array}$ \\
\hline 18 & $\begin{array}{l}\text { Pseudorange corrections } P R_{\operatorname{corr}(n)}(k), \mathrm{B} \text {-values } B_{P R(n, m)}(k) \text { and } \\
\text { MRCC flags }\end{array}$ \\
\hline 19 & EXM-II/MRCC feedback \\
\hline 20 & B-values $B_{P R(n, m)}(k)$ \\
\hline 21 & $\sigma_{n\left(p r n \_g n d\right)}(k)$ \\
\hline 22 & $\sigma_{n\left(p r n \_g n d\right)}(k)$ and $\sigma-\mu$ Monitor flags \\
\hline
\end{tabular}




\subsection{Signal-in-Space Receive and Decode (SISRAD)}

The Signal-in-Space Receive and Decode (SISRAD) function is shown as block A in Figure 6.1. The input data to this block are all the GPS L1 received signals. For the purpose of the present implementation, this set will be restricted to the parameters: pseudoranges $P R_{(i, j)}(k)$ and carrier phases $\phi_{(i, j)}(k)$ from of all GPS satellite ( $i$, representing the satellite Pseudo Random Number PRN) that are detected by each receiver $(j)$ (typically four) at receiver time $(k)$, as well as navigation data. In this work, the set of L1 signal parameters were simulated using the models in Chapter 5. SISRAD converts raw receiver data into an internal data structure suitable for integrity, coherence, and other tests. Also, this monitor includes and recovers the decoded navigation data from the receiver stations to estimate the position of each satellite $x_{i}(k), y_{i}(k)$ and $z_{i}(k)$. Consequently, the elevation angle $\varepsilon_{(i, j)}(k)$ can be estimated using the positions of the receivers.

Each receiver samples GPS signals and provides receiver measurements every $T_{S}=0.5$ seconds in the current GBAS monitor architecture. In other words, the sampling rate is equal to $2 \mathrm{~Hz}$. These GPS measurements are fed into the other GBAS functional blocks for further processing. The output data from this function are navigation data, satellite positions, elevation angles, decoded satellite clock correction data, and lock times, as well as the sets of arranged pseudoranges and carrier phases, referenced to the indices $(i, j, k)$, as appropriate.

\subsection{Signal Quality Receiver (SQR)}

Signal Quality Receiver (SQR) estimates the received signal using $50 \mathrm{~Hz}$ as sampling rate and generates time series of observables to use in Signal Quality Monitoring (SQM) Tests. Also, this monitor estimates the signal power to noise density ratios $C / N_{o(i, j)}(k)$. This monitor is depicted as Block B in Figure 6.1. The input data to this block are received signal powers $C_{(i, j)}(k)$ and the sets of GPS L1 observables described in the previous section. The output data of this block are sets of pseudoranges $P R_{(i, j)}(k)$, carrier phases $\phi_{(i, j)}(k)$, and signal power to noise density ratios $C / N_{o(i, j)}(k)$, referenced to the indices $(i, j, k)$, as appropriate. Additionally, this block can estimate the received signals using a different 
correlator spacing. The functions performed by blocks A and B are carried out by the reference receiver stations located near the runways in the airport.

\subsection{Smooth}

The smoothing function is performed on pseudorange measurements to reduce errors due to noise and high-frequency multipath and is represented as Block D in Figure 6.1. The smoothed pseudorange $P R_{s m(i, j)}(k)$ is obtained for each channel between a pair of satellite $i$ and receiver $j$ at epoch $k$ through a Hatch filter, defined by the following equation

$$
\begin{aligned}
& P R_{\text {sm }(i, j)}(k)=\left(\frac{1}{W_{H}}\right) P R_{(i, j)}(k)+\left(\frac{W_{H}-1}{W_{H}}\right)\left[P R_{s m(i, j)}(k-1)\right. \\
& \left.+\left(\phi_{(i, j)}(k)-\phi_{(i, j)}(k-1)\right)\right]
\end{aligned}
$$

where $P R_{(i, j)}(k)$ is the current raw pseudorange measurement, $P R_{s m(i, j)}(k-1)$ is the previous smoothed pseudorange, $\phi_{(i, j)}(k)$ and $\phi_{(i, j)}(k-1)$ are the current and previous signal carrier phase data. In the above expression, $W_{H}$ is the filter weighting function

$$
W_{H}=\frac{T}{T_{S}}
$$

where $T$ is the filter time constant $(T=100 \mathrm{~s}), T_{S}$ is the sample interval $\left(T_{S}=0.5\right.$ s) of the measurements and it assumed that the satellite is continuously tracked on the receiver. If this is not the case, the filter needs to be reset when receiver $j$ loses phase lock on satellite $i$. In this case, a filter reset flag is sent by the Executive Monitor (EXM). The output data of this monitor are the smoothed pseudoranges $P R_{s m(i, j)}(k)$ and carrier phases $\phi_{(i, j)}(k)$ for each epoch $k$.

\subsection{Measurement Quality Monitoring (MQM)}

The Measurement Quality Monitoring (MQM) function is designed to detect step and impulsive errors on pseudorange $P R_{(i, j)}(k)$, and carrier-phase 
measurements $\phi_{(i, j)}(k)$, due to GPS clock anomalies on the GBAS Ground Facility (GGF) over the last few epochs (LAAS KTA group, 1998; Xie, 2004). This monitor is shown as Block $\mathrm{C}$ in Figure 6.1 and its implementation performs three functions: receiver lock time, carrier-smoothed code innovation, and carrier acceleration-step tests. The matrix $q_{M Q M(i, 3 \cdot(j-1)+m)}(k)$ represents the output data of this monitor. It stores the flags ' $1 / 0$ ' for each test $(m=1,2,3)$, satellite $i$ and receiver $j$ at each epoch $k$. The flag ' 1 ' indicates that the configuration did not pass the corresponding test. Otherwise, the flag marks ' 0 '.

\section{Receiver Lock Time Test}

This test estimates continuous receiver phase lock by computing the residual value between the pseudorange and the carrier-phase observables in comparison with the previous mean value of this difference over a sliding window of $N=10$ previous samples. This test generates a one-bit flag for the Executive Monitor (EXM-I), which will reject data already flagged as invalid. The condition for evaluating the ' $1 / 0$ ' flag is shown in equation (6.3). This prevents the system from continuity loss.

$$
q_{M Q M(i, 3 \cdot(j-1)+1)}(k)=\left\{\begin{array}{l}
1,\left|d_{(i, j)}(k)-m_{d_{(i, j)}}(k-1)\right|>6 S_{d_{(i, j)}}(k-1) \\
0, \quad \text { otherwise }
\end{array}\right.
$$

where $d_{(i, j)}(k)=P R_{(i, j)}(k)-\phi_{(i, j)}(k), m_{d_{(i, j)}}(k-1)$ and $S_{d_{(i, j)}}(k-1)$ are the mean and standard deviation of $d_{(i, j)}(k-1)$ over a sliding window of $N=10$ previous samples, respectively.

It should be advanced that the GBAS monitor re-initializes its carrier smoothing filter described above when the flag is activated, because receiver $j$ loses phase lock on satellite $i$.

\section{Carrier Acceleration-Step Test}

The aim of the carrier acceleration-step test is to detect persistent high acceleration, as well as impulsive, step or other rapid changes in carrier phase measurements. The last 10 continuous epochs (i.e. from epoch $k-9$ to epoch $k$ ) of 
$\phi_{c a(i, j)}(k)$ are calculated for each channel $(i, j)$ at each epoch $\mathrm{k}$, following the next equation

$$
\phi_{c a(i, j)}(k)=\phi_{c(i, j)}(k)-\frac{1}{N_{c}(k)} \sum_{n \in S_{c}(k)} \phi_{c(i, j)}(k)
$$

where $S_{c}(k)$ is the set of $N_{c}(k)$ satellites tracked on receiver $j$ at epoch $k$. The subtraction in Equation 6.4 cancels any possible receiver clock drifts. Additionally, $\phi_{c(i, j)}(k)$ is the carrier phase correction

$$
\phi_{c(i, j)}(k)=\rho_{(i, j)}(k)-\phi_{(i, j)}(k)-c \cdot \Delta t_{s}(k)-\phi_{c i(i, j)}(0)
$$

where $\rho_{(i, j)}(k)$ is the geometric distance between the satellite $i$ and the reference receiver $j$, obtained by equation $(5.4) ; \phi_{(i, j)}(k)$ is the current carrier-phase measurement, $c$ is the speed of light, $\Delta t_{s}(k)$ is the satellite clock error estimate and $\phi_{c i(i, j)}(0)$ is the initial carrier-phase correction.

The ten fitting points estimated from equation (6.5) are used to fit the following quadratic model using the least-squares method (Xie, 2004; Lee, 2005; Koenig, 2010)

$$
\widehat{\phi}_{c a(i, j)}(k, t)=\widehat{\phi}_{0, c a(i, j)}(k, t)+\frac{d \hat{\phi}_{c a(i, j)}(k, t)}{d t} t+\frac{d^{2} \widehat{\phi}_{c a(i, j)}(k, t)}{d t^{2}} \frac{t^{2}}{2}
$$

In the current IMT monitor, $t=0 \mathrm{~s}$ is set at epoch $k-9, t=T_{S}$ is set at epoch $k-8$, consecutively. The least-square method is used to estimate the coefficients of this model. The ramp and acceleration are defined by

$$
\begin{gathered}
\operatorname{Ramp}_{(i, j)}(k)=\frac{d \hat{\phi}_{c a(i, j)}(k, t)}{d t} \\
\operatorname{Acceleration}_{(i, j)}(k)=\frac{d^{2} \hat{\phi}_{c a(i, j)}(k, t)}{d t^{2}}
\end{gathered}
$$


The third test parameter is called step and exposes the apparent change in the latest measurements, being defined by

$$
\operatorname{Step}_{(i, j)}(k)=\phi_{c a(i, j)}(k)-\widehat{\phi}_{c a(i, j)}(k, t)
$$

where $\phi_{c a(i, j)}(k)$ is the value at current epoch $k$ resulting from equation (6.4) and $\widehat{\phi}_{c a(i, j)}(k, t)$ is the predicted value at epoch $k$, estimated from equation (6.6).

If acceleration and step values at the current epoch exceed the associated threshold $E_{\text {th_acce }}=2.6 \mathrm{~cm} / \mathrm{s}^{2}$ or $E_{\text {th_step }}=10 \mathrm{~cm}$, according to the LAAS KTA Group (1998) and Xie (2004), a flag '1' will be issued to the Executive Monitor (EXM-I) as output data of this function. The condition for evaluating the ' $1 / 0$ ' flag is shown in expression (6.10).

$$
q_{M Q M(i, 3 \cdot(j-1)+2)}(k)=\left\{\begin{array}{c}
1,\left|\operatorname{Acce}_{(i, j)}(k)\right|>E_{t_{\text {acce }}} \\
\left|\operatorname{Step}_{(i, j)}(k)\right|>E_{\text {th_step }} \\
0, \quad \text { otherwise }
\end{array}\right.
$$

\section{Carrier-Smoothed Code Innovations Test}

This test is designed to detect impulsive and step errors on raw pseudorange measurements due to anomalies in the signal transmitted by satellite $i$ and detected by receiver $j$. The innovation test is specified by (FAA, 2002; Xie, 2004)

$$
\operatorname{inno}_{(i, j)}(k)=P R_{(i, j)}(k)-\left[P R_{s m(i, j)}(k-1)+\phi_{(i, j)}(k)-\phi_{(i, j)}(k-1)\right]
$$

where $P R_{(i, j)}(k)$ is the current raw pseudorange measurement; $P R_{S m(i, j)}(k-$ 1) is the previous smoothed pseudorange, estimated according to expression (6.1); $\phi_{(i, j)}(k)$ and $\phi_{(i, j)}(k-1)$ are the current and previous carrier phase observable. All these parameters are the input data to this function.

If two or more of the innovation values at three successive epochs exceed associated threshold $E_{t h \_i n}=3.4 \mathrm{~m}$, according to Skidmore and the LAAS KTA Group (1998) and Xie (2004), a flag '1' will be issued to the Executive Monitor 
(EXM-I) as output data of this function. The condition for evaluating the ' $1 / 0$ ' flag is shown in expression (6.12).

$$
q_{M Q M(i, 3 \cdot(j-1)+3)}(k)=\left\{\begin{array}{cc}
1, & \mid \text { inno }_{(i, j)}(k) \mid>E_{t h_{i n}} \\
& \mid \text { inno }_{(i, j)}(k-1) \mid>E_{t h_{i n}} \\
0, & \text { otherwise }
\end{array}\right.
$$

The erroneous code measurement is rejected, but the smoothing filter does not need to be reset.

\subsection{Signal Quality Monitoring (SQM)}

The Signal Quality Monitoring (SQM) function detects and identifies anomalies in the received GPS signal from each satellite, monitor signal power levels and ensure interoperability between different types of receivers in the GBAS operational environment (that is, different ground receivers) (Mitelman, 2000; Xie, 2004). This monitor is represented as Block E in Figure 6.1. The input data to this Block are the set of pseudorange $P R_{(i, j)}(k)$, carrier phase $\phi_{(i, j)}(k)$ and signal power to noise density ratio $C / N_{o(i, j)}(k)$ for each channel $(i, j)$ at each epoch $k$. That is, they coincide with the output data of the SQR Block. This monitor performs the following operations: correlation peak symmetry test, codecarrier divergence test and received signal power test. The matrix $q_{S Q M(i, 3 \cdot(j-1)+m)}(k)$ represents the output data of this monitor, storing the flags ' $1 / 0$ ' for each test $(m=1,2,3)$, satellite $i$ and receiver $j$ at epoch $k$.

\section{Correlation Peak Symmetry Test}

The focus of this test is to ensure that the correlation peak has sufficient symmetry to ensure that the maximum variation in the responses of ground receivers that report $\mathrm{C} / \mathrm{A}$ code correlation measurements at several different correlator spacings is small. These measurements are processed by the SQM monitor to determine if the ideal triangular $\mathrm{C} / \mathrm{A}$ code correlation shape has been appreciably altered by the presence of signal deformations failures (Skidmore and the LAAS KTA Group, 1998). The algorithm starts by defining the residual between pseudorange observables with different correlator spacing described as 


$$
D_{r(i, j)}(k)=P R_{(i, j)}(k)-P R_{N(i, j)}(k)
$$

where $P R_{(i, j)}(k)$ and $P R_{N(i, j)}(k)$ are the pseudorange observables estimated where the correlator spacings are spaced by 1 chip and 0.5 chip, respectively. $P R_{N(i, j)}(k)$ is simulated based on a different contribution of code multipath, using a chip length $\left(\mathrm{T}_{\mathrm{N}}=0.5 \mathrm{~T}_{\mathrm{C} / \mathrm{A}}\right)$ where $\mathrm{T}_{\mathrm{C} / \mathrm{A}}$ is the chip length equivalent to $293 \mathrm{~m}$.

After estimating the residue, it is possible to filter the values of $D_{r(i, j)}(k)$ obtained using the following expression (Skidmore and the LAAS KTA Group, 1998)

$$
D_{s(i, j)}(k)=\left(\frac{1}{W_{H}}\right) D_{r(i, j)}(k)+\left(\frac{W_{H}-1}{W_{H}}\right) D_{s(i, j)}(k-1)
$$

where $W_{H}$ is the filter weighting function described in equation (6.2) and $D_{s(i, j)}(k-1)$ is the previous filtered residue.

If the value of $D_{s(i, j)}(k)$ exceeds the threshold value $E_{t h \_c o}=0.3 \mathrm{~m}$, the flag marks ' 1 ' for this test (that is, it does not pass the test). Otherwise, the flag marks ' 0 ' for each epoch $k$, as indicated by the following expression

$$
q_{S Q M(i, 3 \cdot(j-1)+1)}(k)= \begin{cases}1, & \left|D_{s(i, j)}(k)\right|>E_{t h \_c o} \\ 0, & \text { otherwise }\end{cases}
$$

\section{Code-Carrier Divergence Test}

This test is necessary to facilitate interoperability of receivers from different manufacturers on the ground and in the air. Particularly, the concern is the possibility of different time constants (and filter implementations) used on the ground and in the air. While, it is assumed that these will be standardized, differences in tracking loop implementations within the ground and airborne receivers justify the need for the Code-Carrier Divergence Test. The threshold 4 $\mathrm{m} / \mathrm{min}$ of this algorithm is the basis for air/ground receiver interoperability.

The Code-Carrier Divergence Test uses a Geometric Moving Averaging (GMA) method to estimate the code-carrier divergence (Xie, 2004) described as 


$$
D v g c_{(i, j)}(k)=\frac{\tau_{d}-T_{s}}{\tau_{d}} D v g c_{(i, j)}(k-1)+\frac{1}{\tau_{d}} d z_{(i, j)}(k)
$$

where the time constant for averaging $\tau_{d}$ equals $200 \mathrm{~s}$, the GPS measurement update rate $T_{s}=0.5 \mathrm{~s}$, and $z_{(i, j)}(k)$ is defined as the raw code-minus-carrier measurement

$$
\begin{gathered}
z_{(i, j)}(k)=P R_{(i, j)}(k)-\phi_{(i, j)}(k)=2 I_{(i, j)}(k)+M_{(i, j)}(k)-N_{(i, j)}(k) \lambda \\
d z_{(i, j)}(k)=z_{(i, j)}(k)-z_{(i, j)}(k-1) \\
=2\left[I_{(i, j)}(k)-I_{(i, j)}(k-1)\right]+\left[M_{(i, j)}(k)-M_{(i, j)}(k-1)\right] \\
=\dot{I}_{(i, j)}(k)+\left[M_{(i, j)}(k)-M_{(i, j)}(k-1)\right]
\end{gathered}
$$

where $N_{(i, j)}(k)$ is the integer ambiguity, $M_{(i, j)}(k)$ and $M_{(i, j)}(k-1)$ are the current and previous multipath error. The estimated ionospheric gradient is equal to

$$
\dot{I}_{(i, j)}(k)=\frac{I_{(i, j)}(k)-I_{(i, j)}(k-1)}{T_{S}}
$$

where $I_{(i, j)}(k-1)$ and $I_{(i, j)}(k)$ are the previous and current ionospheric delay. Since $T_{S}=0.5 \mathrm{~s}$, the second and third lines in equation (6.18) numerically coincide. The integer ambiguity $N_{(i, j)}(k)$, is cancelled in equation (6.18), assuming no cycle slips between consecutive samples during the test. If a cycle slip is detected, the GMA method is reset by changing $\operatorname{Dvgc}_{(i, j)}(k-1)=$ $d z_{(i, j)}(k)$ in equation (6.16). If $D v g c_{(i, j)}(k)$ exceeds the threshold value $E_{t h \_c c}=4$ $\mathrm{m} / \mathrm{min}$, the flag marks ' 1 ' for this test (that is, it does not pass the test). Otherwise, the flag marks ' 0 ' for each epoch $k$, as indicated by the following expression

$$
q_{S Q M(i, 3 \cdot(j-1)+2)}(k)=\left\{\begin{array}{cc}
1, & \left|D v g c_{(i, j)}(k)\right|>E_{t h \_c c} \\
0, & \text { otherwise }
\end{array}\right.
$$




\section{Received Signal Power Test}

This test examines the received signal power of each of the GPS satellites. The GPS Standard Positioning Service (SPS) Signal Specification states that the minimum received signal power is $-160 \mathrm{dBW}$ measured at the output of receiving antenna. Assuming a noise density of $-204 \mathrm{dBW}-\mathrm{Hz}$, the received $\mathrm{C} / \mathrm{N}_{0}$ ratio is 44 $\mathrm{dB}-\mathrm{Hz}$ (Lee, 2005). The associated threshold is set at $35 \mathrm{~dB}-\mathrm{Hz}$ to detect when the received signal power is less than one tenth of the minimum power. More specifically, the test is applied to

$$
\left(\frac{C}{N_{0}}\right)_{\operatorname{avg}(i, j)}(k)=\frac{1}{2}\left[\left(\frac{C}{N_{0}}\right)_{(i, j)}(k-1)+\left(\frac{C}{N_{0}}\right)_{(i, j)}(k)\right]
$$

where $\left(C / N_{0}\right)_{(i, j)}(k-1)$ and $\left(C / N_{0}\right)_{(i, j)}(k)$ are the previous and current signal power to noise density ratio. If $\left(C / N_{0}\right)_{\operatorname{avg}(i, j)}(k)$ exceeds the threshold value $E_{t h \_R S P}=35 \mathrm{dBHz}$, the flag marks ' 0 ' for this test (that is, it passed the test). In the opposite case, the flag marks ' 1 ', as summarized by the following expression

$$
q_{S Q M(i, 3 \cdot(j-1)+3)}(k)=\left\{\begin{array}{lc}
1, & \left(C / N_{0}\right)_{\text {avg }(i, j)}(k)<E_{t h \_R S P} \\
0, & \text { otherwise }
\end{array}\right.
$$

Basically, this test evaluates the fading effects due to ionospheric amplitude scintillation in the received signal, simulated according to the statistical model for different geomagnetic latitudes described in Chapter 5. A more restrictive test applies the above test to all 25 consecutive values of: (1) amplitude scintillation records; and (2) phase scintillation records exceeding the threshold $\left(\pi \cdot \mathrm{B}_{\mathrm{w}} / 50\right) \mathrm{rad}$, both generated at the $50 \mathrm{~Hz}$ sampling rate, during each $T_{S}=0.5 \mathrm{~s}$ interval. $\mathrm{B}_{\mathrm{w}}$ is the filter bandwidth.

\subsection{Data Quality Monitoring (DQM)}

Data Quality Monitoring (DQM) verifies that the navigation data for each satellite presents sufficient fidelity. This monitor is represented as Block F in Figure 6.1. The input data to this test are the current satellite ephemerides translated as satellite positions $x_{i}(k), y_{i}(k)$, and $z_{i}(k)$ for each satellite $i$ at each 
epoch $k$ and positions $x_{i(Y e)}(k), y_{i(Y e)}(k)$, and $z_{i(Y e)}(k)$ at the same epoch using ephemeris 24 hours earlier. These data are provided by SISRAD (Block A) and Database (Block L), respectively. In addition, IODE and IODC messages from navigation data are required as input data. To account for possible errors in navigation data, two tests (the Yesterday Ephemerides Minus Today Ephemerides Test and Data Parity Test) have been developed. The matrix $q_{D Q M(i, 2 \cdot(j-1)+m)}(k)$ is the output data from this monitor, which stores the flags ' $1 / 0$ ' for each test $(m=1,2)$, satellite $i$ and receiver $j$ at epoch $k$.

\section{Yesterday Ephemerides minus Today Ephemerides (Ye-Te) Test}

This test is a precise validation of ephemerides for newly risen satellites. It confirms that today's broadcast ephemerides data for each satellite is reliable in comparison with the most recently validated ephemerides data saved as yesterday's ephemerides data in Database. When navigation messages are updated, DQM compares satellite position $x_{i}(k), y_{i}(k), z_{i}(k)$ based on new ephemeris and the satellite positions $x_{i(Y e)}(k), y_{i(Y e)}(k), z_{i(Y e)}(k)$ based on old ephemeris to insure that the new ephemeris is consistent with the old validated over the time-of-ephemeris (toe) 24 hours earlier. (Pullen et al. 2001; LAAS KTA Group,1998)

This test presents flags for each satellite $i$ and epoch $k$. If the residual positions using today's ephemerides and yesterday ephemerides exceeds the error threshold limited to $E_{\text {th_YETE }}=7000 \mathrm{~m}$ for any ECEF coordinate (Skidmore and the LAAS KTA Group, 1998) for each component, the flag ' 1 ' indicates that the configuration did not pass the test. Otherwise, the flag marks ' 0 ', as described in the expression (6.23). Remembering that the index Ye represent the estimations calculated using old ephemerides 24 hours earlier.

$$
q_{D Q M(i, 2 \cdot(j-1)+1)}(k)=\left\{\begin{array}{cc}
1, & \left|x_{i}(k)-x_{i(Y e)}(k)\right|>E_{t h_{-} Y E T E} \\
& \left|y_{i}(k)-y_{i(Y e)}(k)\right|>E_{t h_{-} Y E T E} \\
& \left|z_{i}(k)-z_{i(Y e)}(k)\right|>E_{t h_{-} Y E T E} \\
0, \quad \text { otherwise }
\end{array}\right.
$$


where $x_{i}(k), y_{i}(k), z_{i}(k)$ and $x_{i(Y e)}(k), y_{i(Y e)}(k), z_{i(Y e)}(k)$ are the ECEF satellite positions based on the new ephemeris and old ephemeris.

\section{Data Parity Test}

Data parity test confirms that the IODE and IODC messages of navigation file match and that the data message is consistent across all reference receivers. The IODE broadcast in message subframe 2, the IODE broadcast in message subframe 3, and the IODC in message subframe 1 should match among themselves. This test presents flags for each satellite and epoch $k$. If there is any inconsistency between the described subframes, the flag marks ' 1 ', indicating that the input data did not pass the test. In the opposite case, the flag marks ' 0 ', according to

$$
q_{D Q M(i, 2 \cdot(j-1)+2)}(k)=\left\{\begin{array}{lc}
1, & I O D E_{\text {subframe } 1} \neq I O D E_{\text {subframe } 2} \neq I O D C \\
0, & \text { otherwise }
\end{array}\right.
$$

The output data from this monitor are the satellite positions $x_{i}(k), y_{i}(k)$, and $z_{i}(k)$, in addition to the corresponding DQM flags for each described test, satellite $i$ and epoch $k$.

\subsection{Executive Monitor (EXM)}

The previous sections described each Quality Monitor (QM) algorithm. Each monitor is developed to detect particular failures and generate flags for each channel between satellite $i$ and receiver $j$. The Executive Monitor (EXM) determines which measurements are healthy and is used to generate the GBAS data broadcast, using the flags generated by the QMs. This monitor is represented as Block G in Figure 6.1.

This monitor is divided into two stages. The first (EXM-I), described in the next Section, is designed to select the available channels and observables for the generation of corrections. The second (EXM-II) remove observables based on the interactions with the Multiple Reference Consistency Check (MRCC) Block, the $\sigma-\mu$ Monitor, and Message Field Range Test (MFRT) flags that will be 
characterized in sequence. After their specifications, as well as those of other Blocks, EXM-II will be described.

\subsection{First Stage of the Executive Monitor (EXM-I)}

Each integrity monitor is targeted to detect certain failures and may generate one flag per receiver-satellite pair at each epoch. Once these monitors flag questionable measurements, EXM-I execute several steps of logical reasoning and trial removals determine which failed system elements are the source of the problem (LAAS KTA Group, 1998; Xie et al., 2001). Basically, the input data to the first stage EXM-I are the navigation data, ECEF satellite position, smoothed pseudorange $P R_{s m(i, j)}(k)$, carrier-phase observables $\phi_{(i, j)}(k)$ and the flag matrices $q_{S Q M}(k), q_{D Q M}(k)$ and $q_{M Q M}(k)$ corresponding to each QM monitor.

The first step is to construct two matrices at each epoch to support EXM-I. The first is called tracking matrix $\mathrm{T}(k)$ and the second is called decision matrix $D(\mathrm{k})$. The tracking matrix $\mathrm{T}(k)$ indicates which receivers are tracking which satellites, using the elevation angle mask greater than $20^{\circ}$ to generate flags. The dimension of matrix $\mathrm{T}(k)$ is $\mathrm{N}$ (number of satellites) x M (number of receivers) and each element of this matrix is denoted by a single bit variable 0 or 1 . Its element $t_{(i, j)}(k)=0$ or 1 indicates that satellite $i(i=1, \ldots, \mathrm{N})$ is being tracked by receiver $j(j=1, \ldots, \mathrm{M})$ at epoch $k$ or not, respectively. For the example in Table 6.2, all satellites are tracked by the receivers, with the exception of the case $t_{(2,3)}=1$, where satellite $i=2$ is not tracked by receiver $j=3$.

Table 6.2: Tracking matrix $\mathrm{T}(k)$.

\begin{tabular}{|c|c|c|c|}
\hline & Rec.1 & Rec.2 & Rec.3 \\
\hline Sat 1 & 0 & 0 & 0 \\
\hline Sat 2 & 0 & 0 & 1 \\
\hline$\vdots$ & 0 & 0 & 0 \\
\hline Sat N & 0 & 0 & 0 \\
\hline
\end{tabular}

The decision matrix $\mathrm{D}(k)$ results from operations within the matrix $\mathrm{Q}(k)$ that combines all flags from $q_{S Q M}(k), q_{D Q M}(k)$ and $q_{M Q M}(k)$. One example using 
three receivers is presented in Table 6.3, where QM tests reported faults in a few channels, using ' 1 ' as flag in these cases. Briefly, the SQM table shows two ' $0 / 1$ ' flags for each receiver to indicate results from the Correlation Peak Symmetry, Code-Carrier Divergence and Received Signal Power Tests (' 0 ' represents the regular operation; ' 1 ' represents a failure). The MQM table shows three ' $0 / 1$ ' flags for each receiver to denote results from the Receiver Lock Time, Carrier Acceleration Step and Carrier-Smoothed Code Innovations Tests. The DQM table shows two ' $0 / 1$ ' bits to indicate the results from the Ephemerides Ye-Te and Data Parity Tests.

Table 6.3 shows a failure in the Data Parity Test for channel $(i=1, j=3)$, Correlation Peak Symmetry Test for channel $(i=\mathrm{N}, j=1)$, Code-Carrier Divergence Test for channel ( $i=\mathrm{N}, j=2$ ), and Receiver Lock Time Test for channel $(i=\mathrm{N}, j=1)$.

Table 6.3: Quality matrix $\mathrm{Q}(k)$.

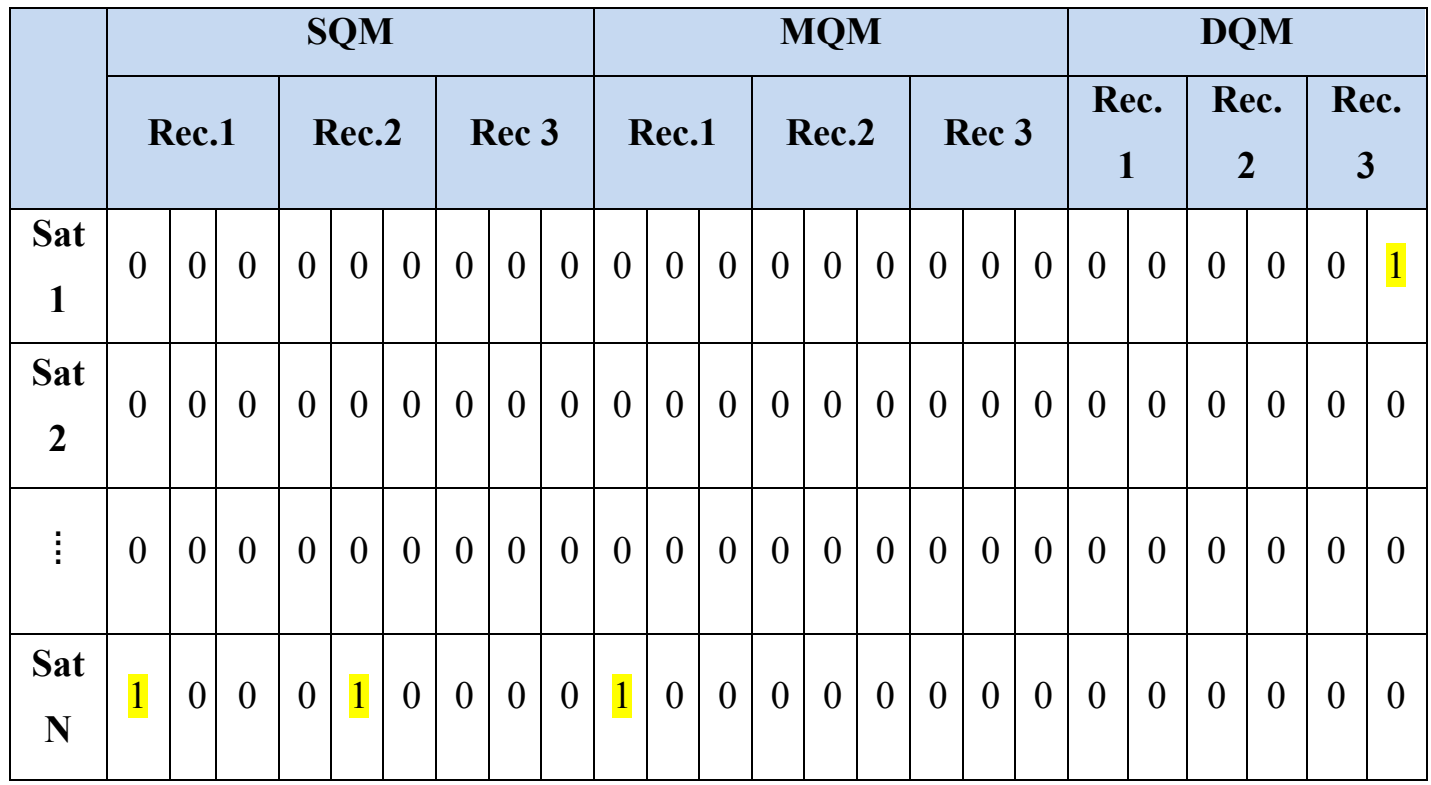

Matrix $\mathrm{Q}(k)$ needs to be stored in memory (and archived if any flags occur), because external maintenance diagnoses of errors will depend on records of exactly which $\mathrm{QM}$ checks were flagged. Matrix $\mathrm{Q}(k)$ is represented by the following mathematical expression 


$$
\begin{aligned}
& Q(k)=\left[q_{S Q M(i, 3 \cdot(j-1)+m)}(k) \cup q_{M Q M(i, 3 \cdot(j-1)+m)}(k)\right. \\
& \left.\cup q_{D Q M(i, 2 \cdot(j-1)+m)}(k)\right]
\end{aligned}
$$

where $q_{S Q M(i, 3 \cdot(j-1)+m)}(k), q_{M Q M(i, 3 \cdot(j-1)+m)}(k)$ and $q_{D Q M(i, 2 \cdot(j-1)+m)}(k)$ are the QM matrix composed by flags for each satellite $i$, receiver $j$ and each test $m$ at epoch $k$.

The next step is construct matrix Q' $(k)$ using the logical OR operation on all ' $0 / 1$ ' bits between each test ( $m=1, m=2$ and $m=3)$ in each matrix $q_{S Q M(i, 3 \cdot(j-1)+m)}(k), \quad q_{M Q M(i, 3 \cdot(j-1)+m)}(k)$ and $q_{D Q M(i, 2 \cdot(j-1)+m)}(k)$, for each fixed pair $(i, j)$. For example, Table 6.4 presents the results using the logical OR operation inside each QM matrix of the previous Table 6.3.

Table 6.4: Matrix Q'(k).

\begin{tabular}{|c|c|c|c|c|c|c|c|c|c|}
\hline & \multicolumn{3}{|c|}{ SQM } & \multicolumn{3}{c|}{ MQM } & \multicolumn{3}{c|}{ DQM } \\
\cline { 2 - 10 } & Rec.1 & Rec.2 & Rec.3 & Rec.1 & Rec.2 & Rec.3 & Rec.1 & Rec.2 & Rec.3 \\
\hline Sat 1 & 0 & 0 & 0 & 0 & 0 & 0 & 0 & 0 & 1 \\
\hline Sat 2 & 0 & 0 & 0 & 0 & 0 & 0 & 0 & 0 & 0 \\
\hline$\vdots$ & 0 & 0 & 0 & 0 & 0 & 0 & 0 & 0 & 0 \\
\hline Sat N & 1 & 1 & 0 & 1 & 0 & 0 & 0 & 0 & 0 \\
\hline
\end{tabular}

Matrix Q' $(k)$ can be represented using the mathematical expression

$$
Q^{\prime}(k)=\left[q_{S Q M(i, j)}^{\prime}(k) \cup q_{M Q M(i, j)}^{\prime}(k) \cup q_{D Q M(i, j)}^{\prime}(k)\right]
$$

where $q_{S Q M(i, j)}^{\prime}(k), q_{M Q M(i, j)}^{\prime}(k)$ and $q_{D Q M(i, j)}^{\prime}(k)$ are the QM matrix composed by flags for each satellite $i$ and receiver $j$ at epoch $k$.

For example, the flags ' 1 ' associated with channels $(N, 1),(N, 2),(N, 4)$ and $(1,3)$ in Table 6.4 represent failures. 
Then, the decision matrix $\mathrm{D}(k)$ is constructed by the application of the logical OR operation to the combination of components of $q_{\operatorname{SQM}(i, j)}^{\prime}(k)$, $q_{M Q M(i, j)}^{\prime}(k)$ and $q_{D Q M(i, j)}^{\prime}(k)$ on matrix Q' $(k)$ for each satellite $i$ and receiver $j$, denoted as ' $1 / 0$ ' flags. The dimension of matrix $\mathrm{D}(k)$ is $\mathrm{N}$ (number of satellites) $\mathrm{x}$ $\mathrm{M}$ (number of receivers). It summarizes the status of each channel, where $d_{(i, j)}(k)=0$ or 1 indicates that the channel between satellite $i(i=1, \ldots, \mathrm{N})$ and receiver $j(j=1, \ldots, \mathrm{M})$ is available or not, respectively. For example, Table 6.5 presents the results from the logical OR operation for each channel from the previous Table 6.4.

Table 6.5: Decision matrix $\mathrm{D}(k)$.

\begin{tabular}{|c|c|c|c|}
\hline & Rec.1 & Rec.2 & Rec.3 \\
\hline Sat 1 & 0 & 0 & 1 \\
\hline Sat 2 & 0 & 0 & 0 \\
\hline$\vdots$ & 0 & 0 & 0 \\
\hline Sat N & 1 & 1 & 0 \\
\hline
\end{tabular}

Consequently, it is possible to construct the $\operatorname{EXM}$ Decision matrix $\operatorname{ED}(k)$ by the application of the logical OR operation to each corresponding component of tracking matrix $\mathrm{T}(k)$ and decision matrix $\mathrm{D}(k)$ described in Table 6.2 and Table 6.5 , respectively. The result of the logical operation can be observed in Table 6.6.

Table 6.6: Decision matrix $\operatorname{ED}(k)$.

\begin{tabular}{|c|c|c|c|}
\hline & Rec.1 & Rec.2 & Rec.3 \\
\hline Sat 1 & 0 & 0 & 1 \\
\hline Sat 2 & 0 & 0 & 1 \\
\hline$\vdots$ & 0 & 0 & 0 \\
\hline Sat N & 1 & 1 & 0 \\
\hline
\end{tabular}

After this process, EXM-I works to isolate cases, by taking the actions described in Table 6.7. This Table summarizes eleven EXM-I isolation cases. It should be observed that the actions are taken sequentially, in the order presented in Table 6.7. That is, isolate case 1 is analyzed first, and so on. 
Table 6.7: Isolate cases and actions.

\begin{tabular}{|c|c|c|}
\hline $\begin{array}{l}\text { Isolate } \\
\text { Cases }\end{array}$ & Description & Action \\
\hline 1 & $\begin{array}{l}\text { one receiver flag on a single } \\
\text { satellite }\end{array}$ & $\begin{array}{l}\text { remove affected satellite on } \\
\text { affected reference receiver only }\end{array}$ \\
\hline 2 & $\begin{array}{l}\text { one satellite flag on more than one } \\
\text { receiver }\end{array}$ & remove affected satellite \\
\hline 3 & $\begin{array}{l}\text { one receiver flag on more than } \\
\text { one satellite }\end{array}$ & remove affected receiver \\
\hline 4 & $\begin{array}{l}\text { one satellite flag on two or more } \\
\text { receivers; one of these receivers } \\
\text { flags on one or more additional } \\
\text { satellites }\end{array}$ & $\begin{array}{l}\text { remove affected receiver and } \\
\text { affected satellite }\end{array}$ \\
\hline 5 & $\begin{array}{l}\text { two or more satellites flag across } \\
\text { three receiver; one receiver } \\
\text { flagging on both satellite }\end{array}$ & 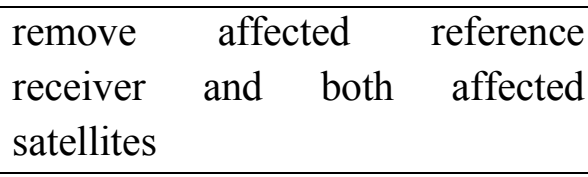 \\
\hline 6 & $\begin{array}{l}\text { two receivers both flag on two or } \\
\text { more satellites }\end{array}$ & remove two reference receivers \\
\hline 7 & $\begin{array}{l}\text { one satellite not tracked by one } \\
\text { receiver }\end{array}$ & no removal is necessary \\
\hline 8 & $\begin{array}{l}\text { satellite not tracked by two or } \\
\text { more receivers }\end{array}$ & remove affected satellite \\
\hline 9 & $\begin{array}{l}\text { satellite not tracked by one } \\
\text { receiver and flagged on another } \\
\text { receiver }\end{array}$ & remove affected satellite \\
\hline 10 & $\begin{array}{l}\text { receiver tracking }<4 \text { common } \\
\text { satellites }\end{array}$ & $\begin{array}{l}\text { receiver is automatically removed } \\
\text { because affects correction } \\
\text { averaging and clock adjustment }\end{array}$ \\
\hline 11 & $\begin{array}{l}\text { receiver tracking }<3 \text { fewer than } \\
\text { total number of satellites tracked }\end{array}$ & $\begin{array}{l}\text { no additional removals are } \\
\text { needed, but receiver behavior is } \\
\text { suspicious }\end{array}$ \\
\hline
\end{tabular}

Basically, EXM-I selects the pseudorange and carrier phase measurements that will be used to generate correction for each available channel, using the above processing of the QM tests.

The next sections of the present work will adopt the indices $(n, m)$ instead of indices $(i, j)$ to indicate available channels after the isolation procedures carried out by the EXM-I monitor. 


\subsection{Correction}

The purpose of the Correction monitor is to generate differential corrections for the smoothed pseudorange $P R_{s m(n, m)}(k)$ and carrier phase $\phi_{(n, m)}(k)$ for each available channel validated by the EXM-I monitor. The correction function is shown as Block $\mathrm{H}$ in Figure 6.1. This and all following functions implicitly assumes that QM tests and EXM-I has extracted all measurements failing QM checks. Thus, it should be stressed that the indices $n$ and $m$ respectively indicate the combinations of all satellites tracked by reference receivers validated by EXM-I at epoch $k$.

The smoothed pseudorange correction $P R_{s m c(n, m)}(k)$ and the carrier phase correction $\phi_{c(n, m)}(k)$ are estimated for each channel associated with satellite $n$ and receiver $m$ at epoch $k$ using

$$
\begin{aligned}
& P R_{s m c(n, m)}(k)=P R_{s m(n, m)}(k)-\rho_{(n, m)}(k)+c \cdot \Delta t_{s}(k) \\
& \phi_{c(n, m)}(k)=\phi_{(n, m)}(k)-\rho_{(n, m)}(k)+c \cdot \Delta t_{s}(k)-\phi_{c i(n, m)}(0)
\end{aligned}
$$

where $P R_{s m(n, m)}(k)$ is the smoothed pseudorange estimated according to Section 6.3, $c$ is the speed of light, $\Delta t_{s}(k)$ is the satellite clock error estimate and $\phi_{c i(n, m)}(0)$ is the initial carrier-phase correction at the first epoch, while $\rho_{(n, m)}(k)$ is the geometric distance between the satellite and the reference receiver, obtained by

$$
\rho_{(n, m)}(k)=\sqrt{\left(x_{n}(k)-x_{m}(k)\right)^{2}+\left(y_{n}(k)-y_{m}(k)\right)^{2}+\left(z_{n}(k)-z_{m}(k)\right)^{2}}
$$

In the above expression, the terms $x, y$ and $z$ with the subscripts $n$ and $m$ correspond, respectively, to the satellite and receiver position in Earth-centered, Earth-fixed (ECEF) coordinates referred to the reception time $k$ (that is, $t_{k}$ ). These input data are extracted from the available channels or the Database (Block P of Figure 6.1) by the EXM-I monitor. It should be noted that the satellite transmit 
time to be used in the estimation of the position $\left(x_{n}(k), y_{n}(k), z_{n}(k)\right)$ is $t_{k}-$ $P R_{\text {sm }(n, m)}(k) / c$.

After the correction procedure, the output data from this monitor are the smoothed pseudorange $P R_{s m c(n, m)}(k)$ and carrier-phase $\phi_{c(n, m)}(k)$ corrections for each available channel $(n, m)$ and epoch $k$ validated by the EXM-I monitor. Recall from Table 2.1 that CAT I has a Vertical Alert Limit (VAL) of 10 meters. Consequently, carrier phase corrections are not required. However, they may be needed for CAT II and III because require VAL of 5.3 meters.

\subsection{Average}

After generating the individual corrections for the available channels validated by EXM-I, receiver clock biases are adjusted to allow measurements to be compared across receivers. The Average monitor, represented by Block I in Figure 6.1, estimates the pseudorange smoothed clock adjustment $P R_{s c a(n, m)}(k)$ and the carrier phase clock adjustment $\phi_{c a(n, m)}(k)$ for each channel $(n, m)$ and epoch $k$ using the following equations:

$$
\begin{gathered}
P R_{s c a(n, m)}(k)=P R_{s m c(n, m)}(k)-\frac{1}{N_{c}(k)} \sum_{n \in S_{c}(k)} P R_{s m c(n, m)}(k) \\
\phi_{c a(n, m)}(k)=\phi_{c(n, m)}(k)-\frac{1}{N_{c}(k)} \sum_{n \in S_{c}(k)} \phi_{c(n, m)}(k)
\end{gathered}
$$

where $P R_{s m c(n, m)}(k)$ represents the smoothed pseudorange correction, $\phi_{c(n, m)}(k)$ is the carrier phase correction, while the indices $n$ and $m$ respectively indicate the combinations of all satellites tracked by reference receivers validated by EXM-I at epoch $k$. Additionally, $S_{c}(k)$ designates the maximum set of $N_{c}(k)$ common satellites tracked by all EXM-I validated reference receivers at epoch $k$. It is assumed that the number of validated reference receivers is larger or equal to 2 . Otherwise, all measurements should be excluded and the system reset. 
In sequence, the averaged broadcast corrections are computed for each satellite using the following equations

$$
\begin{gathered}
P R_{\operatorname{corr}(n)}(k)=\frac{1}{M_{n}(k)} \sum_{m \in S_{m}(k)} P R_{s c a(n, m)}(k) \\
\phi_{\operatorname{corr}(n)}(k)=\frac{1}{M_{n}(k)} \sum_{m \in S_{m}(k)}\left(\phi_{c a(n, m)}(k)-\phi_{c a(n, m)}(0)\right)
\end{gathered}
$$

where $S_{m}(k)$ represents the set of $M_{n}(k)$ reference receivers validated by EXM-I for satellite $n$ and $\phi_{c a(n, m)}(0)$ is evaluated at the first measurement epoch for channel $(n, m)$ to cancel the integer ambiguity.

After these estimations, the output data of the Average monitor are: (1) $P R_{s c a(n, m)}(k)$ and $\phi_{s c a(n, m)}(k)$, the pseudorange smoothed clock and carrierphase clock adjustments for each channel $(n, m)$ and epoch $k$, respectively; and (2) $P R_{\operatorname{corr}(n)}(k)$ and $\phi_{\operatorname{corr}(n)}(k)$, the pseudorange and carrier phase corrections for each satellite $n$ and epoch $k$, respectively. The estimation of the pseudorange corrections are very important for the estimation of the corrected position of the aircraft. The following tests are responsible for validating the pseudorange corrections.

\subsection{Multiple Reference Consistency Check (MRCC)}

The MRCC function is designed to isolate an anomalous receiver that creates large errors in the candidate corrections, being represented as block $\mathrm{J}$ in Figure 6.1 (Lee, 2005). This is accomplished by examining the consistency of corrections for each satellite across all reference receivers by computing B-values, which represent the pseudorange or carrier-phase correction errors for satellite $n$ if reference receiver $m$ has failed. For each satellite and reference receiver, each Bvalue is calculated as the difference between the transmitted pseudorange or carrier-phase correction for that satellite and the same correction calculated excluding that reference receiver (Pullen, 2011; Ferreira, 2007). The B-values can be represented as 


$$
\begin{gathered}
B_{P R(n, m)}(k)=P R_{\operatorname{corr}(n)}(k)-\frac{1}{M_{n}(k)-1} \sum_{\substack{j \in S_{m}(k) \\
j \neq m}} P R_{s c a(n, j)}(k) \\
B_{\phi(n, m)}(k)=\phi_{\operatorname{corr}(n)}(k)-\frac{1}{M_{n}(k)-1} \sum_{\substack{j \in S_{m}(k) \\
j \neq m}}\left(\phi_{c a(n, j)}(k)-\phi_{0(n, j)}(0)\right)
\end{gathered}
$$

where $P R_{\text {corr (n) }}(k)$ represents the broadcast pseudorange correction for the satellite $n, P R_{s c a(n, j)}(k)$ is the pseudorange correction, $\phi_{c a(n, j)}(k)$ is the broadcast carrier-phase correction for the channel $(n, m)$ and $\phi_{0}(n, j)(0)$ is equal to $\phi_{c a(n, j)}(k)$ evaluated at the first measurement epoch obtained using the measurements of reference receiver $m$. Furthermore, $S_{m}(k)$ represents the set of reference receivers that provided valid pseudorange measurements for that satellite and $M_{n}(k)$ is the number of elements of $S_{m}(k)$. It should be remarked that the sum of all B-values from a satellite is equal to zero (FAA, 2002; Xie, 2004). $B_{\phi(n, m)}(k)$ based on carrier-phase measurements is required in CAT II and III, since CAT-I GBAS does not broadcast carrier phase corrections.

Another purpose of this monitor is to report flags to EXM-II, which determines the corrections it will attempt to isolate. First, the MRCC function compares each B-value to its threshold, described in detail by Skidmore and the LAAS KTA Group (1998) and Xie (2004). The following rules can be used to determine whether a channel has a B-value: (1) if $S_{m}(k)$ consists of three receivers, all channels in $S_{m}(k)$ have B-values (with thresholds $\mathrm{D}_{\mathrm{PR}}=0.900 \mathrm{~m}$ and $\left.\mathrm{D}_{\varphi}=0.020 \mathrm{~m}\right)$ and the same holds for satellites tracked by two receivers; (2) if $S_{m}(k)$ consists of two receivers, only channels in $S_{m}(k)$ have B-values (with thresholds $\mathrm{D}_{\mathrm{PR}}=1.600 \mathrm{~m}$ and $\mathrm{D}_{\varphi}=0.035 \mathrm{~m}$ ); and (3) if $S_{m}(k)$ is empty, no pseudorange and carrier phase corrections, as well as B-values exist. In the last case, no GBAS service can be provided at the corresponding epoch.

Results are transmitted in terms of the following MRCC status matrix (for the pseudorange) in the example in the Table 6.8: 
Table 6.8: Matrix $D_{p r}(k)$.

\begin{tabular}{|c|c|c|c|}
\hline & Rec.1 & Rec.2 & Rec.3 \\
\hline Sat 1 & 00 & 00 & 11 \\
\hline Sat 2 & 00 & 00 & 00 \\
\hline$\vdots$ & 00 & 00 & 00 \\
\hline Sat N & 10 & 01 & 00 \\
\hline
\end{tabular}

In Table 6.8, each channel has a combination of two bits: $D_{\operatorname{pr}(n, m)}(k)=$ 00 ; $01 ; 10$; or 11 , indicating $B_{P R(n, m)}(k)$ below threshold; no $B_{P R(n, m)}(k)$ present; $B_{P R(n, m)}(k)$ above threshold; or $B_{P R(n, m)}(k)$ above threshold and maximum for that satellite, respectively. The $D_{p r}(k)$ matrix for pseudorange is used by EXM-II to execute the MRCC isolation procedure. The last three cases in the above list are the first to be removed by the EXM-II isolation procedure. The output data of the MRCC monitor are the sets of $B_{P R(n, m)}(k)$ and $B_{\phi(n, m)}(k)$ values, as well as the $D_{p r}(k)$ and $D_{\phi}(k)$ matrices at each epoch $k$.

\subsection{2. $\sigma-\mu$ Monitor}

Block $\mathrm{K}$ in Figure 6.1 represents the $\sigma-\mu$ Monitor. The inputs of this function are B-values associated to pseudoranges. The purpose of this Block is to estimate the broadcast value for $\sigma_{n\left(p r n \_g n d\right)}(k)$, specified by (Lee, 2005)

$$
\sigma_{n(\text { prn_gnd })}(k)=\sqrt{\frac{\left(a_{0}+a_{1} \cdot e^{-\varepsilon_{n}(k) / \theta_{c}}\right)^{2}}{M_{n}(k)}+\left(a_{2}\right)^{2}}
$$

where $M_{n}(k)$ is the number of reference receivers that are averaged to obtain a differential correction; $\varepsilon_{n}(k)$ is the elevation angle for the $n^{\text {th }}$ satellite; and the parameters $a_{0}, a_{1}, a_{2}$ and $\theta_{c}$ for the applicable Ground Accuracy Designators (GADs) are defined in Table 6.9. The GADs reflect different performance levels of GPS receiver technologies. GAD-A presents a level of performance achievable with low cost installations using a single-aperture antenna and standard correlator. 
GAD-C represent the performance realizable with a narrow correlator and multipath limiting antenna. In addition, GAD-C support GBAS categories II/III precision approaches. GAD-B represents an intermediate level between GAD-A and GAD-C, and it is similar to GAD-C but with a single-aperture antenna instead of multipath-limiting antenna.

Table 6.9: Ground Facility Error allocation model.

\begin{tabular}{|c|c|c|c|c|c|}
\hline \multicolumn{2}{|c|}{$\begin{array}{c}\text { Ground Accuracy } \\
\text { Designator }\end{array}$} & $\boldsymbol{a}_{\mathbf{0}}[\mathrm{m}]$ & $\boldsymbol{a}_{\mathbf{1}}[\mathbf{m}]$ & $\boldsymbol{a}_{\mathbf{2}}[\mathbf{m}]$ & $\boldsymbol{\theta}_{\boldsymbol{c}}\left[{ }^{\circ}\right]$ \\
\hline \multicolumn{2}{|c|}{ GAD-A } & 0.50 & 1.65 & 0.08 & 14.3 \\
\hline \multicolumn{2}{|c|}{ GAD-B } & 0.16 & 1.07 & 0.08 & 15.5 \\
\hline \multirow{2}{*}{ GAD-C } & $\theta_{n} \geq 35^{\circ}$ & 0.15 & 0.84 & 0.04 & 15.5 \\
\cline { 2 - 6 } & $\theta_{n}<35^{\circ}$ & 0.24 & 0 & 0.04 & - \\
\hline
\end{tabular}

Additionally, this Block estimates the ranging error statistics in real time, to ensure that the zero-mean Gaussian distribution defined with the broadcast $\sigma_{n\left(p r n \_g n d\right)}(k)$ overbounds the true error distribution of broadcast differential corrections (Lee, 2005).

The normalized values of $B_{P R(n, m)}(k)$ (that is, B-values divided by their theoretical sigmas $\left.\sigma_{B_{P R}(n)}(k)\right)$ can be estimated by ( Lee et al., 2001)

$$
\begin{gathered}
B_{P R_{\_} n o r m(n, m)}(k)=\frac{B_{P R(n, m)}(k)}{\sigma_{B_{P R}(n)}(k)} \\
\sigma_{B_{P R}(n)}(k)=\frac{\sigma_{n\left(p r n \_g n d\right)}(k)}{\sqrt{M_{n}(k)-1}}
\end{gathered}
$$

where $B_{P R(n, m)}(k)$ are the B-values; $\sigma_{B_{P R}(n)}(k)$ is the standard deviation of the Bvalues; and $M_{n}(k)$ is the number of reference receivers for the satellite $n$. From the normalized $B$-values, it is possible to estimate the sample mean $\hat{\mu}_{P R_{-} n o r m(n, m)}(k)$ and sigma $\hat{\sigma}_{P R_{\_} n o r m(n, m)}(k)$, using the following equations 


$$
\begin{gathered}
\hat{\mu}_{P R_{-} n o r m(n, m)}(k)=\frac{1}{N_{I}(k)-1} \sum_{i=1}^{N_{I}(k)} B_{P R_{-} n o r m(n, m)}(k-i) \\
\hat{\sigma}_{P R_{-} n \operatorname{norm}(n, m)}^{2}=\frac{1}{N_{I}(k)-1} \sum_{i=1}^{N_{I}(k)}\left[B_{P R_{\text {norm }(n, m)}}(k-i)-\hat{\mu}_{P R_{n o r m(n, m)}}(k)\right]^{2}
\end{gathered}
$$

where $N_{I}(k)$ is the number of independent samples used in the estimation at epoch $k$.

According to the Gaussian error model, the estimated sigma has a chi-square distribution with $N_{I}(k)-1$ degrees of freedom and can be described as (Lee, 2005)

$$
\left(N_{I}(k)-1\right) \frac{\hat{\sigma}_{P R_{n o r m}(n, m)}^{2}(k)}{\sigma_{P R_{n o r m}(n, m)}^{2}(k)} \sim \chi^{2}\left\{N_{I}(k)-1\right\}
$$

The interval between independent B-values is expected to be equal to 200 seconds. Consequently, it takes at least one hour to collect 18 independent Bvalues.

The estimated sigma is then compared to this time-dependent threshold, which is lowered as more independent samples are collected. In the same way to MRCC, the $\sigma-\mu$ Monitor builds its own decision matrix $D_{\sigma-\mu}(k)$, generating logic flags for each satellite and reference receiver if the channel passed or failed the test. Then, the $\sigma-\mu$ Monitor flags are resolved in combination with the $D_{\mathrm{PR}, \varphi}(k)$ matrices that exists after MRCC update using logic-OR operations. The output data of this function are $\sigma_{n\left(p r n \_g n d\right)}(k)$ for each satellite $n$ at epoch $k$ and the matrix $D_{\sigma-\mu}(k)$ indicating test pass/fail for B-values.

\subsection{Message Field Range Test (MFRT)}

The function of the Message Field Range Test (MRFT) is to verify that the computed average pseudorange corrections and correction rates fit within confidence bounds (Pullen, 2000; Xie, 2004). This test is executed as the last step 
in EXM before corrections are approved for broadcast. The estimated pseudorange corrections $P R_{\operatorname{corr}(n)}(k)$ from equation (6.36) should be within a threshold of \pm 125 meters and the correction rates $R_{P R_{\operatorname{corr}(n)}}(k)$ should be within a threshold of $\pm 3.4 \mathrm{~m} / \mathrm{s}$ (LAAS KTA group, 1998). The rate of change of $P R_{\operatorname{corr}(n)}(k)$ can be expressed as

$$
R_{P R_{\operatorname{corr}(n)}}(k)=\frac{P R_{\operatorname{corr}(n)}(k)-P R_{\operatorname{corr}(n)}(k-1)}{T_{S}}
$$

where $T_{S}$ is the sample interval $\left(T_{S}=0.5 s\right) ; P R_{\operatorname{corr}(n)}(k)$ and $P R_{\operatorname{corr}(n)}(k-1)$ are the current and previous pseudorange corrections.

If the value obtained of $R_{P R_{\operatorname{corr}(n)}}(k)$ is below of the threshold, the flag marks ' 0 ' and this corresponding correction $P R_{\operatorname{corr}(n)}(k)$ is approved and forwarded to Very High Frequency Data Broadcast (VDB) transmitter. If an MRFT flag ' 1 ' occurs for a given satellite $n$, EXM is alerted, and the satellite is automatically removed for the duration of its overhead pass, due to potential ephemeris fault.

\subsection{Second Stage of the Executive Monitor (EXM-II)}

This section describes the second stage of the Executive Monitor (EXM-II), which performs a series of exclusion steps based on the input data (Pullen, 2000; Xie, 2004): the $D_{p r}(k)$ and $D_{\Phi}(k)$ matrices corresponding to MRCC tests, $\sigma-\mu$ Monitor flags, and MFRT flags. EXM-II generates a $(N x M)$ MRCC decision matrix $\mathrm{D}_{\mathrm{MRCC}}(k)$ based on a logical OR operation between $D_{P R}(k)$; and MRCC flow chart resolution that is described in detail in LAAS KTA Group (1998). It is similar to the decision matrix $\operatorname{EID}(k)$ of the first stage EXM-I. It should be remembered that the first $\operatorname{EID}(k)$ elements result from logic-OR operations. Hence, this monitor assesses an updated $\operatorname{EIID}(k)$ matrix that will represent the isolate procedure for the EXM-II monitor.

In other words, the single matrix $D_{M R C C}(k)$ is conformed using logical OR operations between $D_{P R}(k)$ and $D_{\Phi}(k)$ for CAT II and III. In case of CAT I the 
matrix $D_{M R C C}(k)$ is represented as $D_{P R}(k)$. After this operation, it is possible to repeat this procedure by the application of the logical OR to $D_{M R C C}(k)$ and $D_{\sigma-\mu}(k)$ resulting in a $\operatorname{EXM-II~matrix} \operatorname{EIID}(k)$. An example of the result is described in Table 6.10.

Table 6.10: Matrix $\operatorname{EIID}(k)$.

\begin{tabular}{|c|c|c|c|}
\hline & Rec.1 & Rec.2 & Rec.3 \\
\hline Sat 1 & 0 & 0 & 1 \\
\hline Sat 2 & 0 & 0 & 0 \\
\hline$\vdots$ & 0 & 0 & 0 \\
\hline Sat N & 1 & 1 & 0 \\
\hline
\end{tabular}

Accordingly, each $\operatorname{EIID}(k)$ flag marks ' 1 ' indicates that the pseudorange correction for the corresponding satellite should be excluded. As an example, Table 6.10 shows that the satellite $n=1$ and satellite $n=\mathrm{N}$ are excluded and there are no pseudorange corrections for these satellites. Summarizing, EXM has the role of assessing the failures in measurements performed for satellite-receiver channels. Based on ground system algorithms, only healthy results contribute to corrections that will be transmitted to aircrafts.

\subsection{Protection Levels}

Section 2.3.2 presented a brief introduction to the horizontal and vertical protection levels HPL and VPL, which bounds the horizontal and vertical position errors, respectively, with a confidence level derived from the integrity risk requirement (RTCA, 2008). These protection levels are estimated in the second stage of the EXM monitor. The present Section closely follows the description provided by (ICAO, 1999).

This section specifies algorithms for the calculation of the protection levels for aircraft landing that, basically, depend on the position of the aircraft at each epoch $k$. They will be subdivided into vertical and horizontal protection levels $\operatorname{VPL}(k)$ and $\operatorname{HPL}(k)$, using the following equations 


$$
\begin{aligned}
& V P L(k)=K_{f f m d} \sqrt{\sum_{i=1}^{N} S_{i(\text { vert })}^{2}(k) \cdot \sigma_{i}^{2}(k)} \\
& H P L(k)=K_{f f m d} \sqrt{\sum_{i=1}^{N} S_{i(\text { horiz })}^{2}(k) \cdot \sigma_{i}^{2}(k)}
\end{aligned}
$$

where $K_{f f m d}$ is the quantile of a unit Gaussian distribution corresponding to $\gamma$ ( $\gamma$ differs by application and is on the order of $10^{-7}$ to $10^{-10}$ ). The values of $K_{f f m d}$ calculated for CAT I precision approach and approach procedures with vertical guidance (APV) are given in Table 6.11 for different numbers of valid reference receivers.

Table 6.11: Value of the multiplier $K_{f f m d}$.

\begin{tabular}{|c|c|c|c|}
\hline & \multicolumn{3}{|c|}{$M_{j}$ reference receivers } \\
\hline Multiplier & $\mathbf{2}$ & $\mathbf{3}$ & $\mathbf{4}$ \\
\hline $\boldsymbol{K}_{\text {ffmd }}$ & 5.762 & 5.810 & 5.847 \\
\hline
\end{tabular}

In the above equation, $i$ is the index for one of the satellites used in the position solution; $N$ is the number of satellites used in the position solution; $S_{i(v e r t)}(k)$ and $S_{i(\text { horiz) }}(k)$ are contributions of satellite $i$ to the vertical and horizontal position estimates to the approach track, expressed as

$$
\begin{gathered}
S_{i(\text { vert })}(k)=S_{3, i}(k)+S_{1, i}(k) \cdot \tan \theta_{G P A} \\
S_{i(\text { horiz })}(k)=\sqrt{S_{1, i}^{2}(k)+S_{2, i}^{2}(k)}
\end{gathered}
$$

In expressions (6.49) and (6.50), $\theta_{G P A}$ is the glide path angle (typically $3^{\circ}$ ); $S_{1, i}$, $S_{2, i}(k)$ and $S_{3, i}(k)$ are the $i^{\text {th }}$ element (satellite) of the $1^{\text {st }}, 2^{\text {nd }}$ and $3^{\text {rd }}$ row of the matrix of protection $(\mathrm{S})$, defined as

$$
S(k)=\left(G^{T}(k) W(k) G(k)\right)^{-1} G^{T}(k) W(k)
$$


where $G(k)$ is the observation matrix consisting of $N$ rows of unit line-of-sight vectors from each satellite to the receiver antenna, augmented by a ' 1 ' for the clock. That is, the $i^{\text {th }}$ row of $G$ corresponds to the $i^{\text {th }}$ satellite in view and can be written in terms of the azimuth and elevation angles $A z_{i}(k)$ and $\varepsilon_{i}(k)$, respectively, calculated from the aircraft position at epoch $k$. This matrix is nondimensional, being defined as

$$
G(k)=\left[\begin{array}{cccc}
-\cos \varepsilon_{1} \cos A z_{1}(k) & -\cos \varepsilon_{1} \operatorname{sen} A z_{1}(k) & -\operatorname{sen} \varepsilon_{1}(k) & 1 \\
-\cos \varepsilon_{2} \cos A z_{2}(k) & -\cos \varepsilon_{2} \operatorname{sen} A z_{2}(k) & -\operatorname{sen} \varepsilon_{2}(k) & 1 \\
\vdots & \vdots & \vdots & 1 \\
-\cos \varepsilon_{N} \cos A z_{N}(k) & -\cos \varepsilon_{N} \operatorname{sen} A z_{N}(k) & -\operatorname{sen} \varepsilon_{N}(k) & 1
\end{array}\right]
$$

Additionally, $W(k)$ is the weighting matrix, expressed as

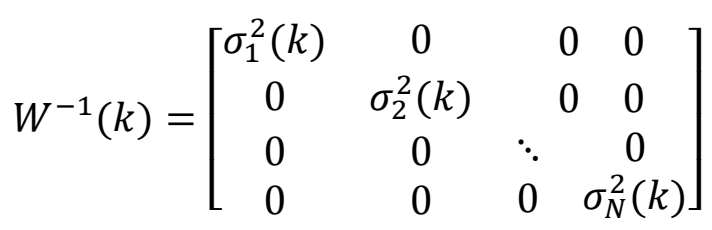

Implicit in the specification of the weighting matrix $W(k)$ is the assumption that the measurement errors from different satellites are zero-mean, uncorrelated, and Gaussian-distributed random variables; otherwise, such a characterization would be very difficult, in general. For each measurement, the error model is given by

$$
\sigma_{i}(k)=\sqrt{\sigma_{i(\text { air })}^{2}(k)+\sigma_{i(t r o p o)}^{2}(k)+\sigma_{i(\text { iono })}^{2}(k)+\sigma_{i\left(p r n \_g n d\right)}^{2}(k)}
$$

where $i$ is the index for one of the satellites used in the position solution; $\sigma_{i \text { (air) }}(k)$ is the airborne error, determined from the receiver noise estimate and the specified multipath model; $\sigma_{i(\text { tropo })}(k)$ and $\sigma_{i(\text { iono })}(k)$ are introduced by the residual tropospheric and ionospheric errors respectively; and $\sigma_{i \text { (prn_gnd) }}(k)$ is the ground error, which includes the estimation of ground station receiver noise and multipath error. The last term in equation (6.54) has 
already been characterized by expression (6.40) (see section 6.12). Each of the other terms will be specified next.

\subsubsection{Airborne Pseudorange Error}

The overall airborne accuracy model is computed as follows

$$
\sigma_{i(\text { air })}(k)=\sqrt{\sigma_{i(\text { noise })}^{2}(k)+\sigma_{i(\text { multipath })}^{2}(k)}
$$

In this expression, $\sigma_{i(n o i s e)}(k)$ is the error due to wideband noise and interference, including receiver and thermal noise, inter-channel biases, extrapolation and processing errors, being modeled as

$$
\sigma_{i(\text { noise })}(k)=a_{0 n}+a_{1 n} \cdot e^{-\varepsilon_{i}(k) / \theta_{c n}}
$$

where $\varepsilon_{i}(k)$ is the elevation angle for the $i^{t h}$ satellite at epoch $k$, while $a_{0 n}, a_{1 n}$ and $\theta_{c n}$ for the applicable Airborne Accuracy Designator (AAD) are defined in Table 6.12. The AADs were designed to indicate different performance levels of GPS receiver technologies (McGraw et al., 2000).

Table 6.12: Airborne error model parameters.

\begin{tabular}{|c|c|c|c|}
\hline $\begin{array}{c}\text { Airbone } \\
\text { Accurancy Designator }\end{array}$ & $\boldsymbol{a}_{\mathbf{0 n}}[\mathbf{m}]$ & $\boldsymbol{a}_{\mathbf{1} \boldsymbol{n}}[\mathrm{m}]$ & $\boldsymbol{\theta}_{\boldsymbol{c n}}{ }{ }^{{ } \mathbf{]}}$ \\
\hline AAD-A & 0.15 & 0.43 & 6.9 \\
\hline AAD-B & 0.11 & 0.13 & 4.0 \\
\hline
\end{tabular}

Additionally, $\sigma_{i \text { (multipath) }}(k)$ is the error due to airframe multipath, being estimated by

$$
\sigma_{i \text { (multipath })}(k)=0.13+0.53 e^{-\varepsilon_{i}(k) / 10^{\circ}}
$$




\subsubsection{Differential Tropospheric Delay Error}

The differential tropospheric delay error is estimated from the expression

$$
\sigma_{i(\text { tropo })}(k)=\sigma_{N}(k) \cdot h_{o}(k) \frac{10^{-6}}{\sqrt{0.002+\sin ^{2} \varepsilon_{i}(k)}}\left(1-e^{-\Delta h(k) / h_{o}(k)}\right)
$$

where $\sigma_{N}(k)$ is the (unitless) refractivity uncertainty and $h_{o}(k)$ is the tropospheric scale height, both available from the GBAS Type 2 message; $\Delta h(k)$ is the height of the aircraft above the GBAS reference point (m); and $\varepsilon_{i}(k)$ is the elevation angle for the $i^{\text {th }}$ satellite at epoch $k$.

The GBAS Ground Facility (GGF) is responsible for providing broadcast parameters $\left(\sigma_{N}, h_{o}\right.$ and $\left.\Delta h\right)$ that are consistent with prevailing conditions at the site. If this real time estimation process is to be avoided, then these parameters must be set to constant values that cover the worst case conditions expected during operations.

\subsubsection{Ionospheric Residual Uncertainty}

The ionospheric residual uncertainty is estimated from the expression

$$
\sigma_{i(\text { iono })}(k)=F_{p p}(k) \cdot \sigma_{\text {vert_iono_grad }}(k) \cdot\left(X_{\text {air }}(k)+2 \tau v_{\text {air }}(k)\right)
$$

where $F_{p p}(k)$ is the vertical-to-slant obliquity factor; $\sigma_{\text {vert_iono_grad }}(k)$ is the standard deviation of a Gaussian distribution associated with the residual ionospheric uncertainty due to spatial decorrelation (a parameter provided by the ground subsystem in the GBAS Type 2 message); $X_{\text {air }}(k)$ is the distance (slant range) between the aircraft and the GBAS reference point $(\mathrm{m}) ; \tau$ is the time constant of the smoothing filter $(100 \mathrm{~s})$; and $v_{\text {air }}(k)$ is the horizontal speed of the aircraft $(\mathrm{m} / \mathrm{s})$.

In expression (6.59), $\sigma_{\text {vert_iono_grad }}(k)$ is an important parameter that depends on the variation of the ionospheric delay. This delay is affected by 
irregular ionospheric disturbances that presents spatial variation. In Chapter 4 were estimated vertical ionospheric gradients and the corresponding standard deviation $\sigma_{\text {vert_iono_grad }}$ using the station-pair method in order to identify periods with high activity. In this work are used these estimations to evaluate the extended spatial ionospheric gradient, each value will be used for all satellites estimations.

\subsection{Very High Frequency (VHF) Data Broadcast (VDB) Transmitter}

The VDB formats and transmits GBAS correction messages to the landing aircraft in the protected aeronautical radionavigation band $(108.000 \mathrm{MHz}-$ 117.950 MHz). The information that is received and is transmitted from this block are the epoch, correction to the pseudorange $P R_{\operatorname{corr}(n)}(k)$ and B-value $B_{P R(n, m)}(k)$ for each satellite; aircraft protection levels, $\sigma_{n\left(p r n \_g n d\right)}(k)$, reference location, local tropospheric data, power, and identification of the station levels.

In the present implementation, the aircraft will use the standalone GPS information to estimate its position. Next, the GBAS pseudorange corrections $P R_{\operatorname{corr}(n)}(k)$ associated with the satellites (when available) will be used by the aircraft to refine its position estimation. Since the true position of the aircraft is assumed to be known, the two positioning errors (with or without the GBAS corrections) may be compared.

\subsection{Estimation of the Aircraft Position}

The aircraft position in ECEF coordinates $\left(x_{j}(k), y_{j}(k), z_{j}(k)\right)$ and the clock offset $\tau_{j}(k)$ at each epoch $k$ is estimated using the iterative point positioning method. Following Kaplan and Hegarty (1996), this procedure requires the linearization of the pseudorange measurements from at least four satellites to estimate the aircraft coordinates. This process is based on the following expression for different channels

$$
P R_{i}(k)=\left\|\vec{s}_{i}(k)-\vec{r}_{a}(k)\right\|+c \tau(k)
$$

$$
P R_{i}(k)=\sqrt{\left[x_{i}(k)-x(k)\right]^{2}+\left[y_{i}(k)-y(k)\right]^{2}+\left[z_{i}(k)-z(k)\right]^{2}}+c \tau(k)(6.61)
$$


where $\vec{r}_{a}(k)=(x(k), \mathrm{y}(k), \mathrm{z}(k))$ represent the aircraft position and its ECEF coordinates; $\tau(k)$ is the aircraft clock offset; $\vec{s}_{i}(k)=\left(x_{i}(k), \mathrm{y}_{i}(k), \mathrm{z}_{i}(k)\right)$ indicate satellite $i$ position and its ECEF coordinates; and $i$ ranges from 1 to the number of available satellites. As before, epoch $k$ is the receiver time, while $\vec{s}_{i}(k)$ is estimated at the associated transmission time. Basically, the left-hand sides of equations (6.60) and (6.61) are compact versions of equation (5.1). In the current equations (6.60) and (6.61), the pseudorange can be compactly represented as a nonlinear function of the coordinates and clock offset of the aircraft receiver

$$
P R_{i}(k)=f_{i}(x(k), y(k), z(k), \tau(k)) .
$$

These variables that are the objective of the estimation, considering the factors that affect the signal propagation: ionospheric delay and scintillation, tropospheric delay, multipath, clock offsets and random errors, included in the left-hand side of equation (6.62), which is modeled as described in Chapter 5.

Let the estimated receiver position $(x(k), \mathrm{y}(k), \mathrm{z}(k))$ differ from an approximate position $(\hat{x}(k), \hat{y}(k), \hat{z}(k))$ by a displacement $(\Delta x(k), \Delta y(k), \Delta z(k))$.

Using the approximate position $(\hat{x}(k), \hat{y}(k), \hat{z}(k))$ and time bias $\hat{\tau}(k)$, an approximate pseudorange can be calculated

$$
\widehat{P R}_{i}(k)=f_{i}(\hat{x}(k), \hat{y}(k), \hat{\mathrm{z}}(k), \hat{\tau}(k))
$$

$\widehat{P R}_{i}(k)=\sqrt{\left[x_{i}(k)-\hat{x}(k)\right]^{2}+\left[y_{i}(k)-\hat{y}(k)\right]^{2}+\left[z_{i}(k)-\hat{z}(k)\right]^{2}}+c \hat{\tau}(k)(6.64)$

The unknown aircraft position and receiver clock offset to be estimate consist of the sum of the approximate and incremental components, which be represented by

$$
\begin{aligned}
x(k) & =\hat{x}(k)+\Delta x(k) \\
y(k) & =\hat{y}(k)+\Delta y(k) \\
z(k) & =\hat{z}(k)+\Delta z(k) \\
\tau(k) & =\hat{\tau}(k)+\Delta \tau(k)
\end{aligned}
$$


Combining equations (6.62) and (6.65), it is possible to write

$$
\begin{gathered}
P R_{i}(k) f_{i}(x(k), \mathrm{y}(k), \mathrm{z}(k), \tau(k))=f_{i}(\hat{x}(k)+\Delta x(k), \hat{y}(k)+\Delta y(k), \\
\hat{z}(k)+\Delta z(k), \hat{\tau}(k)+\Delta \tau(k))
\end{gathered}
$$

This function can be expanded about the approximate point and associated receiver clock offset $(\hat{x}(k), \hat{y}(k), \hat{z}(k), \hat{\tau}(k))$ through Taylor's theorem, ignoring the second and higher order terms, to obtain

$$
\begin{aligned}
& f_{i}(\hat{x}(k)+\Delta x(k), \hat{y}(k)+\Delta y(k), \hat{z}(k)+\Delta z(k), \hat{\tau}(k)+\Delta \tau(k)) \\
& =f_{i}(\hat{x}(k), \hat{y}(k), \hat{z}(k), \hat{\tau}(k))+\frac{\partial f_{i}(\hat{x}(k), \hat{y}(k), \hat{z}(k), \hat{\tau}(k))}{\partial \hat{x}(k)} \Delta x(k) \\
& +\frac{\partial f_{i}(\hat{x}(k), \hat{y}(k), \hat{z}(k), \hat{\tau}(k))}{\partial \hat{y}(k)} \Delta y(k)+\frac{\partial f_{i}(\hat{x}(k), \hat{y}(k), \hat{z}(k), \hat{\tau}(k))}{\partial \hat{z}(k)} \Delta z(k) \\
& +\frac{\partial f_{i}(\hat{x}(k), \hat{y}(k), \hat{z}(k), \hat{\tau}(k))}{\partial \hat{\tau}(k)} \Delta \tau(k)
\end{aligned}
$$

Respectively substituting the first two terms in equation (6.67) by the left-hand sides of equations (6.62) and (6.63), estimating the partial derivatives in equation (6.67) with the help of equation (6.64), one obtains

$$
\begin{aligned}
P R_{i}(k) & =\widehat{P R}_{i}(k)-\frac{x_{i}(k)-\hat{x}(k)}{\hat{\rho}_{i}(k)} \Delta x(k)-\frac{y_{i}(k)-\hat{y}(k)}{\hat{\rho}_{i}(k)} \Delta y(k) \\
& -\frac{z_{i}(k)-\hat{z}(k)}{\hat{\rho}_{i}(k)} \Delta z(k)+c \Delta \tau(k)
\end{aligned}
$$

where $\hat{\rho}_{i}(k)$ is the first term in the right-hand side of equation (6.64).

The resulting system of linear equations (for $N$ available satellites) can be written in matrix form

$$
A(k)\left[\begin{array}{c}
\Delta x(k) \\
\Delta y(k) \\
\Delta z(k) \\
c \Delta \tau(k)
\end{array}\right]=\left[\begin{array}{c}
\Delta P R_{1}(k) \\
\Delta P R_{2}(k) \\
\vdots \\
\Delta P R_{N}(k)
\end{array}\right]=\left[\begin{array}{c}
P R_{1}(k)-\widehat{P R}_{1}(k) \\
P R_{2}(k)-\widehat{P R}_{2}(k) \\
\vdots \\
P R_{N}(k)-\widehat{P R}_{N}(k)
\end{array}\right]
$$


where the design matrix $A(k)$ is

$$
A(k)=\left[\begin{array}{ccccc}
\frac{-\left(x_{1}(k)-\hat{x}(k)\right)}{\hat{\rho}_{1}(k)} & \frac{-\left(y_{1}(k)-\hat{y}(k)\right)}{\hat{\rho}_{1}(k)} & \frac{-\left(z_{1}(k)-\hat{z}(k)\right)}{\hat{\rho}_{1}(k)} & 1 \\
\frac{-\left(x_{2}(k)-\hat{x}(k)\right)}{\hat{\rho}_{2}(k)} & \frac{-\left(y_{2}(k)-\hat{y}(k)\right)}{\hat{\rho}_{2}(k)} & \frac{-\left(z_{2}(k)-\hat{z}(k)\right)}{\hat{\rho}_{2}(k)} & 1 \\
\vdots & \vdots & \vdots & \vdots \\
\frac{-\left(x_{N}(k)-\hat{x}(k)\right)}{\hat{\rho}_{N}(k)} & \frac{-\left(y_{N}(k)-\hat{y}(k)\right)}{\hat{\rho}_{N}(k)} & \frac{-\left(z_{N}(k)-\hat{z}(k)\right)}{\hat{\rho}_{N}(k)} & 1
\end{array}\right]
$$

The above system of "linearized observation equations" is solved by the leastsquares method to estimate the incremental components

$$
\left[\begin{array}{c}
\Delta x(k) \\
\Delta y(k) \\
\Delta z(k) \\
c \Delta \tau(k)
\end{array}\right]=\left(A^{T} A\right)^{-1} A^{T}\left[\begin{array}{c}
P R_{1}(k)-\widehat{P R}_{1}(k) \\
P R_{2}(k)-\widehat{P R}_{2}(k) \\
\vdots \\
P R_{N}(k)-\widehat{P R}_{N}(k)
\end{array}\right]
$$

The ECEF coordinates of the aircraft position are estimated by

$$
\left[\begin{array}{c}
x(k) \\
y(k) \\
z(k) \\
c \tau(k)
\end{array}\right]=\left[\begin{array}{c}
\hat{x}(k)+\Delta x(k) \\
\hat{y}(k)+\Delta y(k) \\
\hat{z}(k)+\Delta z(k) \\
c \hat{\tau}(k)+c \Delta \tau(k)
\end{array}\right]
$$

This method is iterative, as described next. Each value in the set $\left\{P R_{i}(k)\right\}$ is modeled according to the procedures in Chapter 5, assuming that the aircraft reference position $\vec{r}_{r e f}(k)=\left(x_{r e f}(k), y_{r e f}(k), z_{r e f}(k)\right)$ along its approach path at epoch $k$. This set of pseudodistances remains fixed through all iterations. The initial approximate position $(\hat{x}(k), \hat{y}(k), \hat{z}(k))$ could be any point close to the corresponding airport. For example, the position of a GBAS reference receiver. The initial approximate time bias $\hat{\tau}(k)$ is set to zero. This initial approximate parameters $(\hat{x}(k), \hat{y}(k), \hat{z}(k), \hat{\tau}(k))$ are used in equation (6.64) to determine the corresponding sets of values $\left\{\hat{\rho}_{i}(k)\right\}$ and $\left\{\widehat{P R}_{i}(k)\right\}$. 
Substituting the values in the previous paragraph into equations (6.69) to (6.72), current estimations for the position coordinates $(x(k), \mathrm{y}(k), \mathrm{z}(k))$ and receiver clock offset $\tau(k)$ are obtained. The currently estimated vector $(x(k), \mathrm{y}(k), \mathrm{z}(k), \tau(k))$ is substituted for $(\hat{x}(k), \hat{y}(k), \hat{\mathrm{z}}(k), \hat{\tau}(k))$ in: (1) equation (6.64), to update the corresponding sets of values $\left\{\hat{\rho}_{i}(k)\right\}$ and $\left\{\widehat{P R}_{i}(k)\right\}$; and (2) equations (6.69) to (6.71) and in the right-hand side of equation (6.72) to provide the next vector of estimates.

The purpose of the iterations is to achieve better approximations in the estimation of the above parameters. For this reason, all increments should simultaneously become less than $0.01 \mathrm{~m}$ for convergence to be achieved in the present work

$$
\Delta x(k), \Delta y(k), \Delta z(k), c \Delta \tau(k)<0.01 m
$$

To estimate GBAS effects on the aircraft position estimation, the left-hand side of

$$
P R_{\text {corrected }(i)}(k)=P R_{\text {smoothed }(i)}(k)-P R_{\text {corr }(i)}(k)
$$

is substituted for the pseudorange $P R_{\text {smoothed }(i)}(k)$ associated with the path between available GPS satellite $i$ and the aircraft in the above iterative procedure. In equation (6.74), the pseudorange correction $P R_{\operatorname{corr}(i)}(k)$ is estimated by GBAS using equation (6.36).

In both cases, the aircraft position errors are estimated by the difference between the final estimated position and its reference position along the flight path

$$
\vec{E}_{p o s}(k)=\left[\begin{array}{l}
E_{x}(k) \\
E_{y}(k) \\
E_{z}(k)
\end{array}\right]=\left[\begin{array}{l}
x(k)-x_{r e f}(k) \\
y(k)-y_{r e f}(k) \\
z(k)-z_{r e f}(k)
\end{array}\right]
$$


where $(x(k), \mathrm{y}(k), \mathrm{z}(k))$ are the ECEF coordinates of the aircraft estimated position; $\left(x_{r e f}(k), y_{r e f}(k), z_{r e f}(k)\right)$ are the ECEF coordinates of the aircraft reference position, both at epoch $k$. Naturally, the error vector can also be expressed in different reference frames, such as (north, east, down), by proper transformations.

$$
\vec{E}_{\text {pos }}(k)=\left[\begin{array}{c}
E_{\text {north }}(k) \\
E_{\text {east }}(k) \\
E_{\text {down }}(k)
\end{array}\right]=R^{T}\left[\begin{array}{c}
E_{X}(k) \\
E_{Y}(k) \\
E_{Z}(k)
\end{array}\right]
$$

where $\mathrm{R}$ is the rotation matrix and can be expressed as

$$
R=\left[\begin{array}{ccc}
-\sin (\Phi(k)) \cos (\lambda(k)) & -\sin (\lambda(k)) & -\cos (\Phi(k)) \cos (\lambda(k)) \\
-\sin (\Phi(k)) \sin (\lambda(k)) & \cos (\lambda(k)) & -\cos (\Phi(k)) \sin (\lambda(k)) \\
\cos (\Phi(k)) & 0 & -\sin (\Phi(k))
\end{array}\right]
$$

where the $\Phi(k)$ and $\lambda(k)$ are the geodetic latitude and longitude of the aircraft position at each epoch $k$, respectively.

Also, these errors can be presented in horizontal and vertical positions errors (HPE and VPE) because are more intuitive and practical for navigation than ECEF and Geodetic coordinates. The HPE and VPE are estimated from the position error in North, East and Down components using the following equations

$$
\begin{aligned}
& \operatorname{HPE}(k)=\sqrt{E_{\text {east }}^{2}(k)+E_{\text {north }}^{2}(k)} \\
& \operatorname{VPE}(k)=E_{\text {down }}(k)
\end{aligned}
$$

The described methodology will be applied to different cases of interest characterized by the geomagnetic latitude (scenario and flight data), season and solar activity. 


\section{Results from the GBAS Simulation Model}

Chapter 5 introduced the methodology for the simulation of time series of the signals in space, represented by the pseudorange, carrier phase, and received signal power. These observables were generated for particular case studies, to evaluate the magnitude of each component (due to the ionosphere, troposphere, multipath, etc.) and their time variations. Chapter 6 introduced processes for monitoring different aspects of quality of the signal in space for each channel and generating corrections and additional parameters, using a GBAS simulation model. Also, the positioning algorithm was described.

Chapter 7 presents results from simulation studies based on the Rio de Janeiro and Fortaleza Airports for days with low and high solar activity. These airports were selected because they are located at different geomagnetic latitudes. The next Sections describe, for each scenario, the positions of the GBAS receivers, the flight data and the differential corrections estimated for each channel using the GBAS monitor algorithms. The horizontal and vertical positioning errors are calculated based on uncorrected and smoothed/corrected pseudoranges (combination of uncorrected pseudoranges and differential corrections).

\subsection{Aircraft Positioning at the Rio de Janeiro/Galeão Antonio Carlos Jobim International Airport, Brazil}

In this section, the positions of approach and landing of one aircraft to the Rio de Janeiro/Galeão Antonio Carlos Jobim International Airport (SBGL; in short, Rio de Janeiro Airport) located in Rio de Janeiro, Brazil, are estimated during a commercial flight, to evaluate the performance of the GBAS simulation model under different geophysical conditions. This airport was selected due to its location in the poleward side of the southern crest of the equatorial ionization anomaly (EIA), generally located within the geomagnetic latitude interval $\left(-12^{\circ},-20^{\circ}\right)$. 
To analyze the ionospheric effects during a particular flight, results from two case studies are reported. The prevailing ionospheric conditions during the case studies can be summarized by analyzed periods of time: (1) 00:00:00 to 00:10:00 UTC on 20 December 2014 (high solar activity during the summer solstice); and (2) 00:00:00 to 00:10:00 UTC on 22 July 2009 (low solar activity during the winter solstice). More detailed descriptions on the environment will be provided in the corresponding Sections.

\subsubsection{Scenario}

Since 2011, the Brazilian Departmento de Controle do Espaço Aéreo (DECEA), in cooperation with the Federal Aviation Administration (FAA), has been making efforts to evaluate the performance of GBAS in the Brazilian airspace. The behavior of a GBAS station in Rio de Janeiro is of great interest, considering that it is located in a region of low latitude, subject to intense ionospheric activity, with the occurrence of phenomena such as severe gradients, plasma bubbles and scintillation. For these reasons, a Honeywell SLS-4000 SmartPath station was acquired and installed at the Rio de Janeiro Airport, to evaluate the performance of system already certified by the FAA for unrestricted use in mid-latitude environments.

The GBAS installed at the Rio de Janeiro Airport is composed by the main GBAS station and four reference receivers. The receivers RSMU1, RSMU2 RSMU3 and RSMU4 are deployed around the runway of the Rio de Janeiro Airport, as shown in Figure 7.1. The geodetic coordinates and DIP latitude of each receiver antenna are indicated in Table 7.1.

Table 7.1: Positions of receiver antennas at the Rio de Janeiro Airport.

\begin{tabular}{|c|c|c|c|}
\hline Receiver Station & Latitude & Longitude & DIP latitude \\
\hline RSMU1 & $22.7991^{\circ} \mathrm{S}$ & $43.2502^{\circ} \mathrm{W}$ & $20.9021^{\circ} \mathrm{S}$ \\
\hline RSMU2 & $22.8017^{\circ} \mathrm{S}$ & $43.2497^{\circ} \mathrm{W}$ & $20.9043^{\circ} \mathrm{S}$ \\
\hline RSMU3 & $22.8011^{\circ} \mathrm{S}$ & $43.2483^{\circ} \mathrm{W}$ & $20.9047^{\circ} \mathrm{S}$ \\
\hline RSMU4 & $22.7988^{\circ} \mathrm{S}$ & $43.2488^{\circ} \mathrm{W}$ & $20.9027^{\circ} \mathrm{S}$ \\
\hline
\end{tabular}




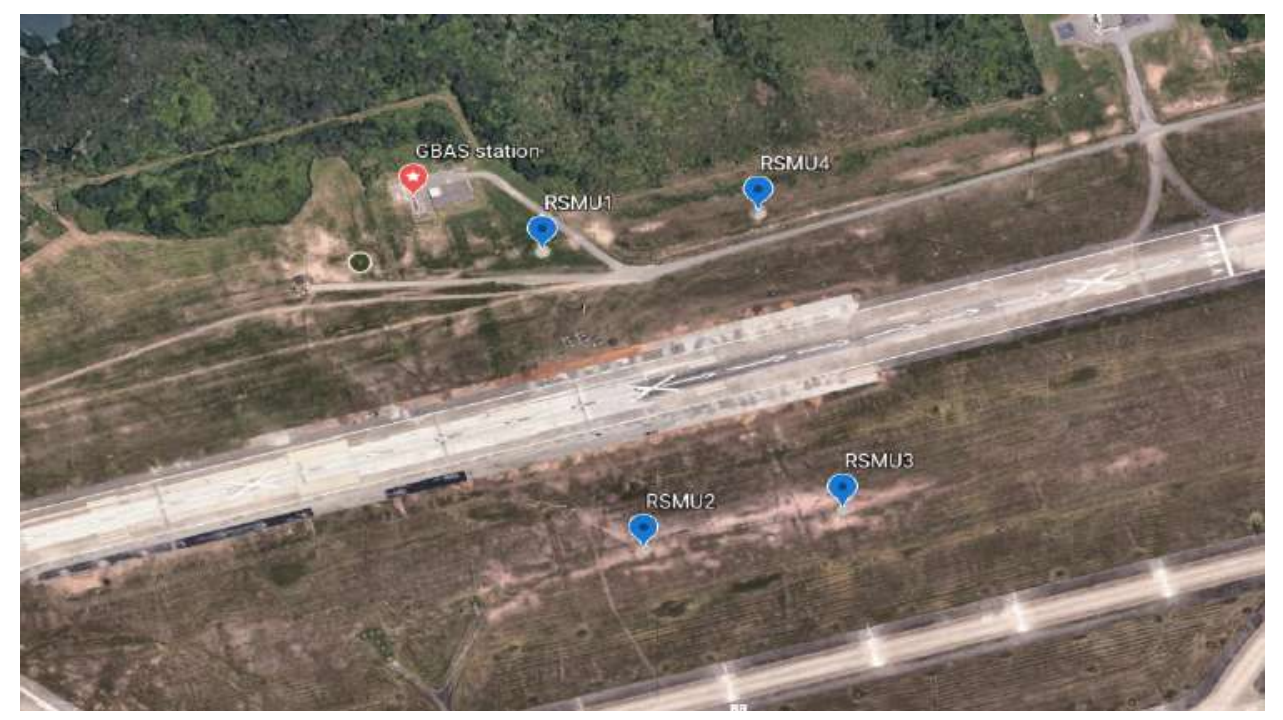

Figure 7.1: Positions of the GBAS station and reference antennas at the Rio de Janeiro Airport [adapted from Google Earth].

\subsubsection{Flight Data}

This work uses the approach and landing information of one aircraft to the Rio de Janeiro Airport. The latitude, longitude, altitude and speed data of a commercial flight (TAM/JJ3839) operated by LATAM airlines were used and transformed into ECEF coordinates. This flight occurred on 12 March 2018, departing from the Recife International Airport (SBRF). Table 7.2 provides more information on the selected flight and Figure 7.2 shows the last section of the flight path. The flight data (positions and velocities), previously explored by Pereira (2018), can be accessed on the FlightAware website (https:/pt.flightaware.com).

Table 7.2: Information on the TAM/JJ3839 flight.

\begin{tabular}{|c|c|}
\hline Flight & TAM/JJ3839 \\
\hline Date & 12 March 2018 \\
\hline Aircraft & Airbus A321 \\
\hline Departure Airport & Recife International Airport \\
\hline Actual Departure Time & $06: 28: 23$ UTC \\
\hline Arrival Airport & Rio de Janeiro International Airport \\
\hline Actual Arrival Time & $08: 57: 34$ UTC \\
\hline
\end{tabular}




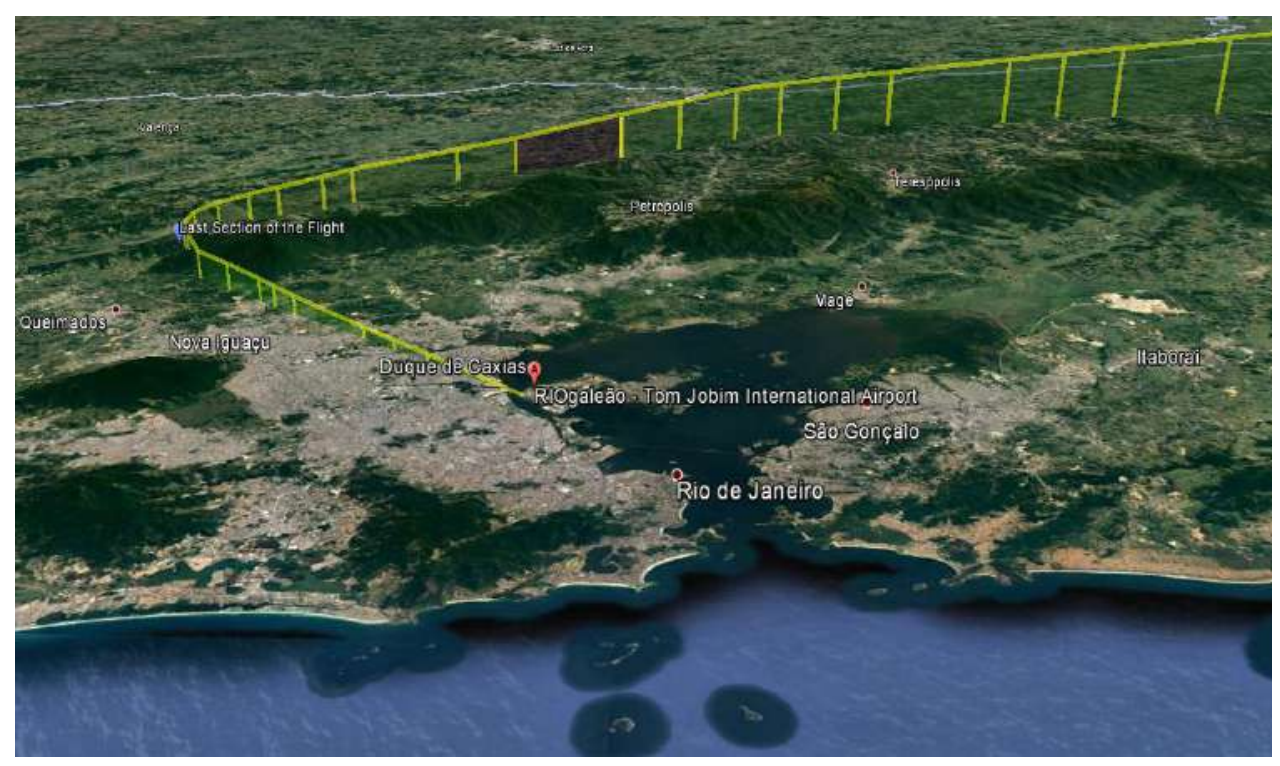

Figure 7.2: Last section of TAM/JJ3839 flight path [adapted from Google Earth].

Figure 7.2 identifies the tridimensional last path section of the TAM/JJ3839 flight (approximately 28 kilometers), before the aircraft landing at the Rio de Janeiro Airport by the yellow line limited between the blue and red markers. The last section of the flight path presents a time interval between 08:52:34 UTC and 08:57:34 UTC. Thus, the positioning results will be based on pseudoranges, the combination of pseudoranges and GBAS corrections, as well as on information on the true position of the aircraft during the last 5 minutes of the flight path that corresponds to the precision approach categories: APV I, CAT-I, CAT-II and CATIII, according to ICAO. The present work will simulate two aircraft landings, each one with 5 minutes, representing a total interval of 10 minutes, emphasizing CATI requirements.

In addition, the aircraft flight profiles have been characterized by parameters such as aircraft speed and altitude for the last section of the TAM/JJ3839 flight, as illustrated in Figure 7.3, which shows how both parameters decrease during the last section of the flight, as expected. The information on the aircraft speed is used to estimate the ionospheric residual uncertainty presented in equation (6.59) and the height information is used to estimate the tropospheric delay error in equation (6.58). 

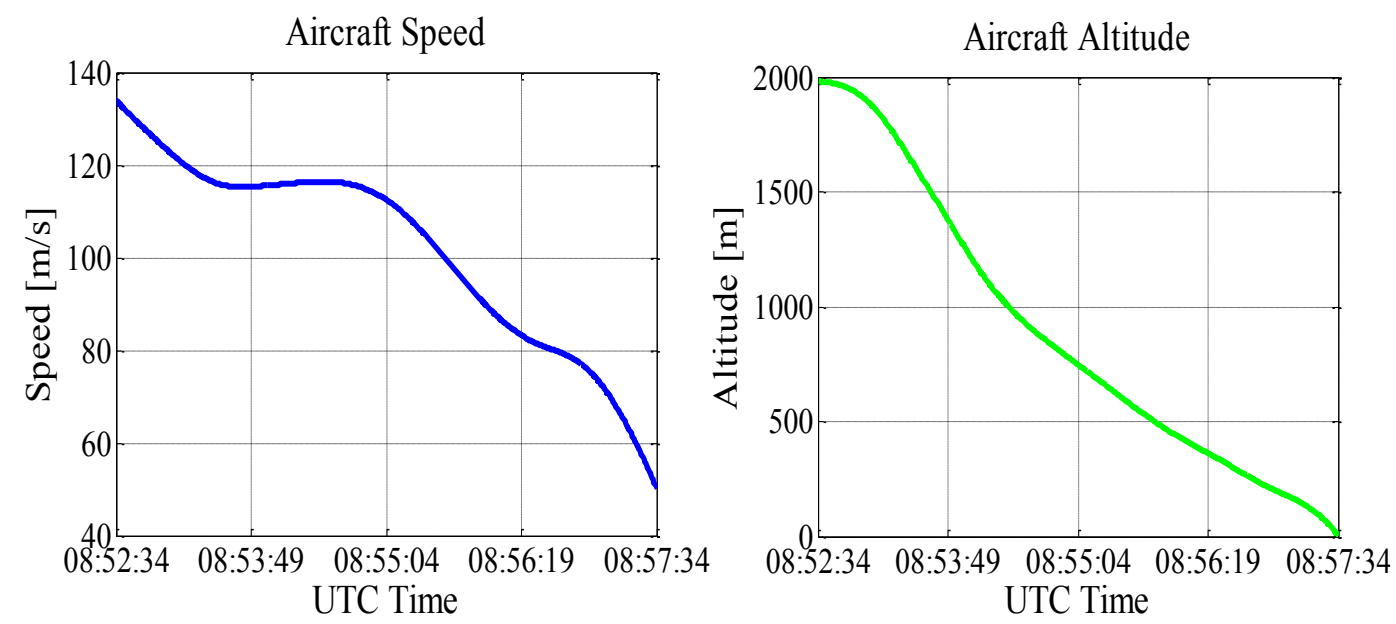

Figure 7.3: Aircraft speed (Left) and aircraft altitude (Right) of Flight

TAM/JJ3839 between 08:52:34 UTC and 08:57:34 UTC.

\subsubsection{Case study: Rio de Janeiro Airport, 20 December 2014}

The sequence of implemented algorithms is briefly described using equations from Chapter 5 and Chapter 6 as references. The main program is composed by algorithms that were developed in MATLAB and the subroutine for the adapted IRI Model was programmed in FORTRAN. The input data for the main program are the time interval, GPS navigation and flight data, reference receiver positions, as well as environmental and system parameters.

The first step is the estimation of ECEF satellite coordinates $x_{i}(k)$, $y_{i}(k), z_{i}(k)$ and the satellite clock error $\Delta t_{s(i)}(k)$ for all available GPS satellites $i$ between 00:00:00 UTC and 00:10:00 UTC on 20 December 2014 at the associated transmission time, using the GPS navigation data, following the method described in Annex A to Chapter 6. In this study, only satellites with elevation angles greater than $20^{\circ}$ are taken into account for the estimations, for consistency with the associated limitations of the amplitude scintillation measurements. For example, in the current case study, the aircraft tracked 6 GPS satellites during the described flight time interval (SV 15, SV 16, SV 18, SV 21, SV 22 and SV 29). The estimated azimuth and elevation of each satellite can be observed in the skyplot in the left panel of Figure 7.4. The right panel of the same Figure shows the ground projections of the GPS satellite position, using the same color code and the corresponding IPPs, using red dots. 

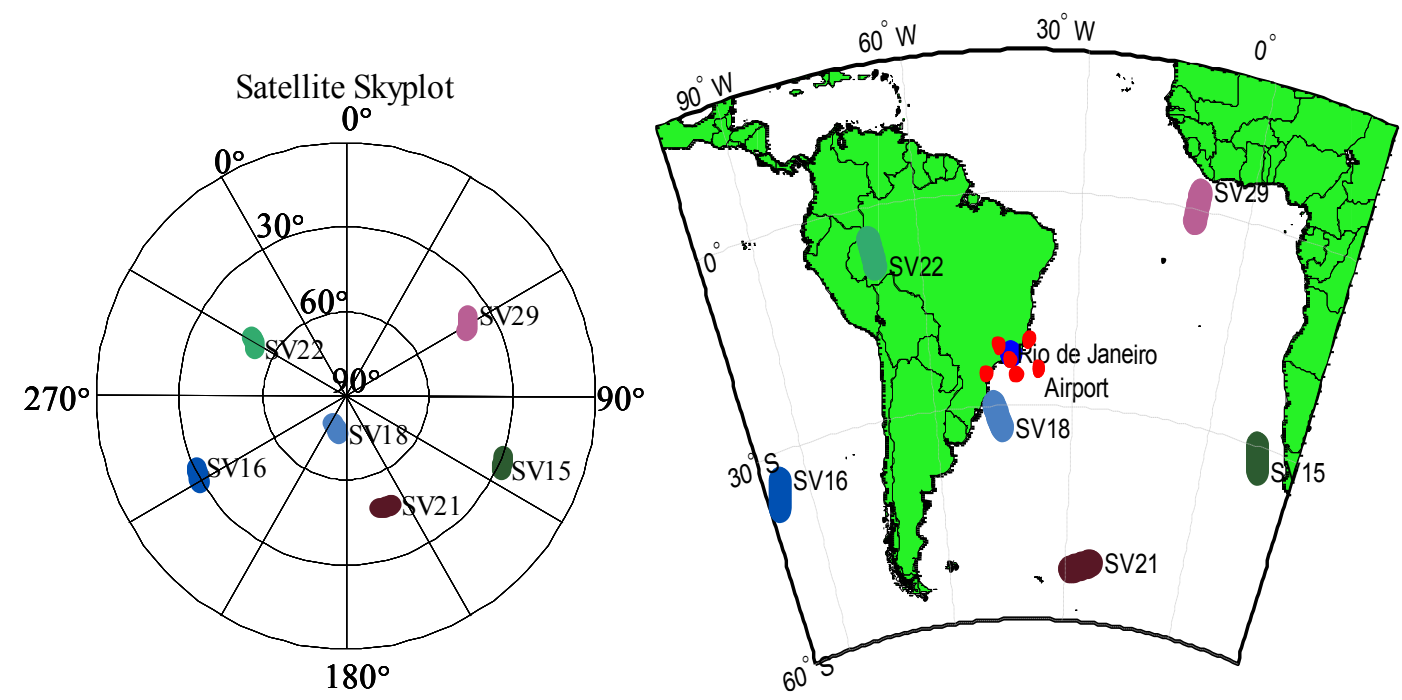

Figure 7.4: GPS satellite skyplot for TAM/JJ3839 flight from 00:00:00 UTC to 00:10:00 UTC on 20 December 2014 (Left); ground projections of the GPS satellite positions using the same color code and the corresponding IPPs using red dots (Right).

Then, time series of pseudorange $P R_{(i, j)}(k)$, carrier-phase $\phi_{(i, j)}(k)$, and received signal power $C_{(i, j)}(k)$ observables were generated for channels defined between tracked GPS satellites ( $i=\mathrm{SV} 15, \mathrm{SV} 16, \mathrm{SV} 18, \mathrm{SV} 21, \mathrm{SV} 22$ and SV 29) and the GBAS reference receiver antenna and aircraft positions $(j)$ between 00:00:00 UTC and 00:10:00 UTC on 20 December 2014, with time denoted by $(k)$. These observables were simulated based on the satellite positions using the models described in equations (5.1) to (5.3), respectively. The sampling rate for the pseudorange and the carrier phase observables are $T_{s}=0.5 \mathrm{~s}$, while that for the received signal power observables is $T_{s}=0.02 \mathrm{~s}$, corresponding to the sampling frequency $50 \mathrm{~Hz}$, for a high time-resolution consideration of ionospheric amplitude and phase scintillation effects.

Modeling of the ionospheric delay $I_{(i, j)}(k)$; tropospheric delay $T_{(i, j)}(k)$; multipath effects $m_{\mathrm{PR}(i, j)}(k), m_{\phi(i, j)}(k)$, and $m_{\mathrm{C}(i, j)}(k)$ associated with pseudorange, carrier phase and amplitude, as well as the effective isotropic radiated power of each satellite transmitter $\operatorname{EIRP}_{(i, j)}(k)$ are based on environmental, geophysical and system parameters listed in Tables 7.3 to 7.5 , respectively, as well as on the formulation described in Chapter 5. 
Table 7.3: Geophysical parameters on 20 December 2014.

\begin{tabular}{|c|c|c|}
\hline & Parameter & Value \\
\hline \multirow{3}{*}{$\begin{array}{l}\text { Geophysical } \\
\text { parameters }\end{array}$} & DoY & 354 \\
\cline { 2 - 3 } & F10.7 index & $196.7[\mathrm{sfu}]$ \\
\cline { 2 - 3 } & Kp index & 1.7 \\
\hline
\end{tabular}

Table 7.4: Environment parameters at Rio de Janeiro Airport on 20 December 2014.

\begin{tabular}{|c|c|c|}
\hline \multirow{4}{*}{ Environment } & Parameter & Value \\
\hline & Air pressure & $1010[\mathrm{hPa}]$ \\
\cline { 2 - 3 } & Temperature & $26^{\circ} \mathrm{C}$ \\
\cline { 2 - 3 } & Humidity & $83 \%$ \\
\cline { 2 - 3 } & Dielectric constant $\varepsilon_{r}$ (wet ground) & 30 \\
\cline { 2 - 3 } & Conductivity $\sigma$ & $0.20 \mathrm{~S} / \mathrm{m}$ \\
\cline { 2 - 3 } & Refractivity $N$ & 370 \\
\cline { 2 - 3 } & Refractivity Uncertainy & 15 \\
\cline { 2 - 3 } & Tropospheric scale height & $12900 \mathrm{~m}$ \\
\hline
\end{tabular}

Table 7.5: Simulation system parameters.

\begin{tabular}{|c|c|c|}
\hline \multirow{4}{*}{$\begin{array}{c}\text { Parameter } \\
\text { System } \\
\text { Description }\end{array}$} & Value \\
\cline { 2 - 3 } & Gosses in the transmitter path & $16.25 \mathrm{dBW}$ \\
\cline { 2 - 3 } & Gain of the transmitter antenna & $13.50 \mathrm{~dB}$ \\
\cline { 2 - 3 } & Gain of the receiver antenna & $3 \mathrm{~dB}$ \\
\cline { 2 - 3 } & Time smoothing filter & $293 \mathrm{~m}$ \\
\cline { 2 - 3 } & GBAS Accuracy Designator & $100 \mathrm{~s}$ \\
\cline { 2 - 3 } & Airbone Accuracy Designator & 'C' \\
\hline & Glide Slope Angle & $3^{\circ}$ \\
\hline
\end{tabular}

At every integer minute, the vector of amplitude scintillation indices $\mathrm{S}_{4}$ for the available satellites were sorted according to the distribution associated with the 
label SM-HG (scintillation season from September to March, for high solar activity) displayed in Figure 5.39. Each vector remained constant during the corresponding minute, as shown in Figure 7.5. All the simulated scintillation indices $\mathrm{S}_{4}$ are limited to the interval $0.1 \leq \mathrm{S}_{4} \leq 0.9$. The corresponding vectors of standard deviation of phase scintillation $\sigma_{\varphi}$, are sorted from the corresponding values of $\mathrm{S}_{4}$ as prescribed at the end of Section 5.1.6, in association with Figure 5.10. The corresponding time series of $\sigma_{\varphi}$ vectors are represented in Figure 7.6. It should be observed that the highest values observed for SV 18 in Figure 7.6 exceed those reported by Moraes et al. (2017), reproduced in Figure 5.10.

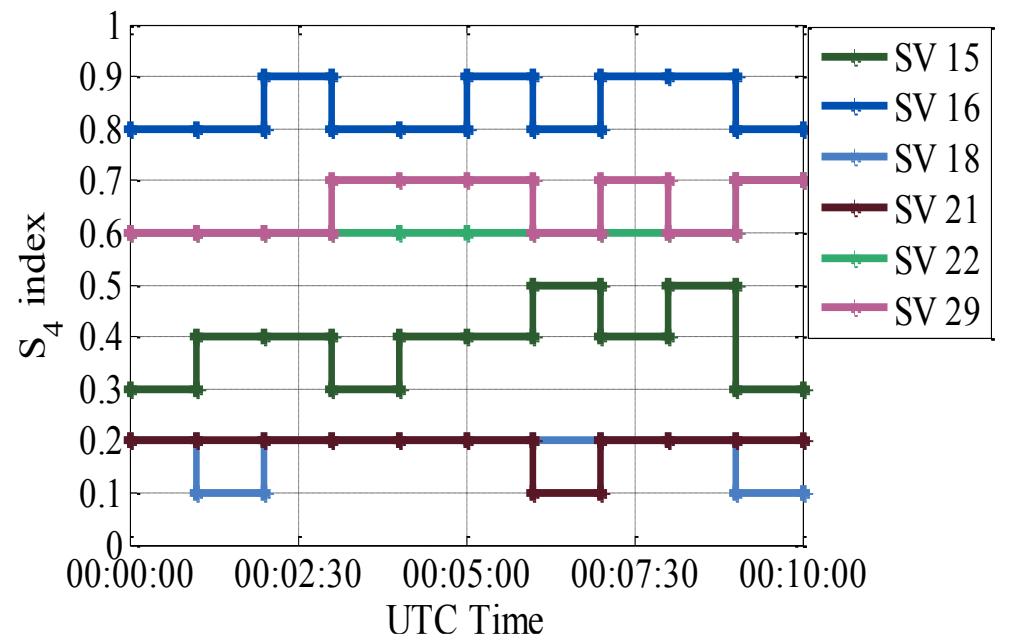

Figure 7.5: Time series of amplitude scintillation index $\mathrm{S}_{4}$ for each GPS satellite, with 1-min resolution.

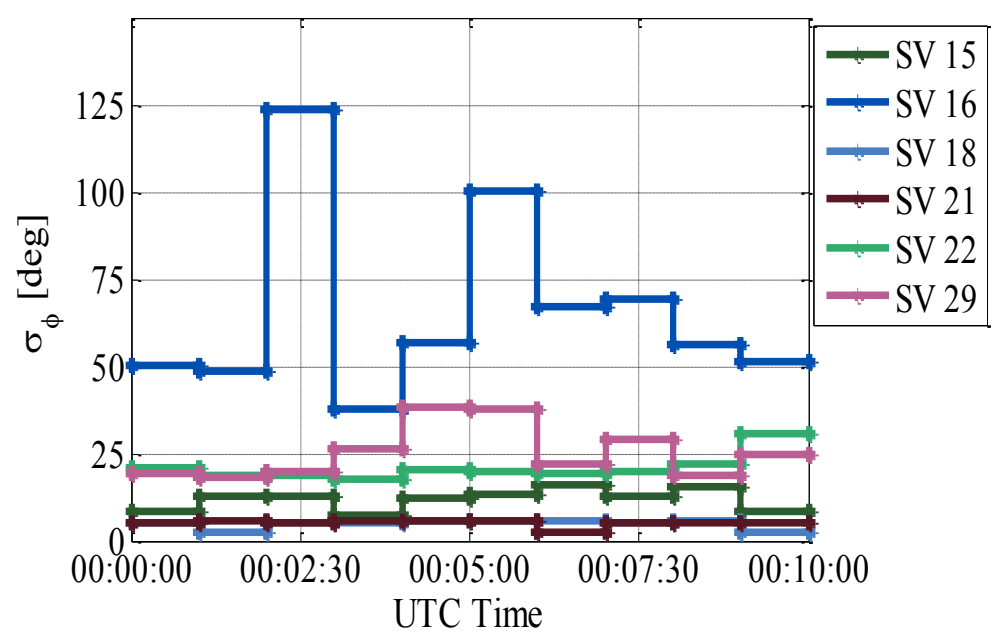

Figure 7.6: Time series of standard deviation of phase scintillation $\sigma_{\varphi}$ for each GPS satellite, with 1-min resolution. 
It is important to note that the present work also used ionospheric gradients $\left(\Delta I / d_{I P P}\right)$ to represent spatial variations of the ionosphere. Series of gradients are extracted from the estimated ionospheric delays carried out between RIOD and ONRJ using the Station-Pair method $\left(g^{s}\right)$ during the same interval of time, as presented in Section 4.3. These gradients are added to the ionospheric delay model $I_{(i, j)}(k)$ for the corresponding channel between the aircraft and GPS satellites, considering the distance between IPP aircraft $_{\text {and IPP }}$ RSMU1.

Figures 7.7 to 7.9 display individual 10-min (subdivided into two 5-min intervals, for the first and second approaches) time series of pseudorange, carrierphase and received signal power effects due to the parameters listed above (that is, ionospheric and tropospheric delays, multipath, etc.), respectively, generated for two aircraft receivers during two landings, as described above. Each time series (for each tracked satellite) is represented by different line color.

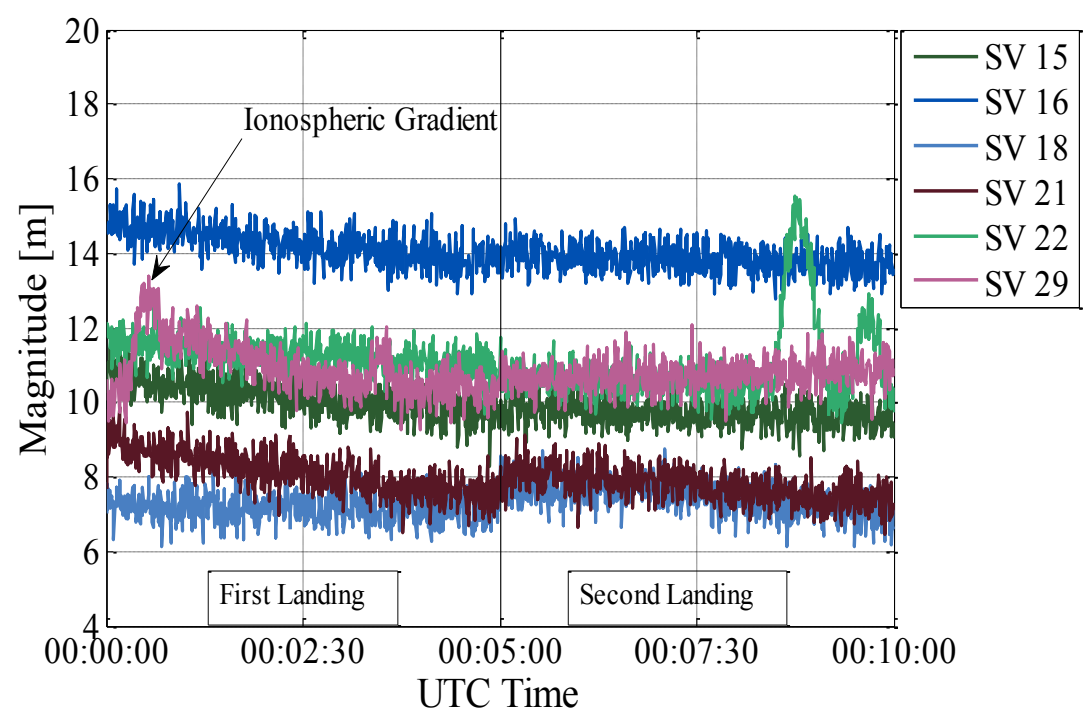

Figure 7.7: Time series of the effects due to ionospheric and tropospheric delays, multipath and random errors on the pseudorange, generated for TAM/JJ3839 flight from 00:00:00 UTC and 00:10:00 UTC on 20 December 2014. 


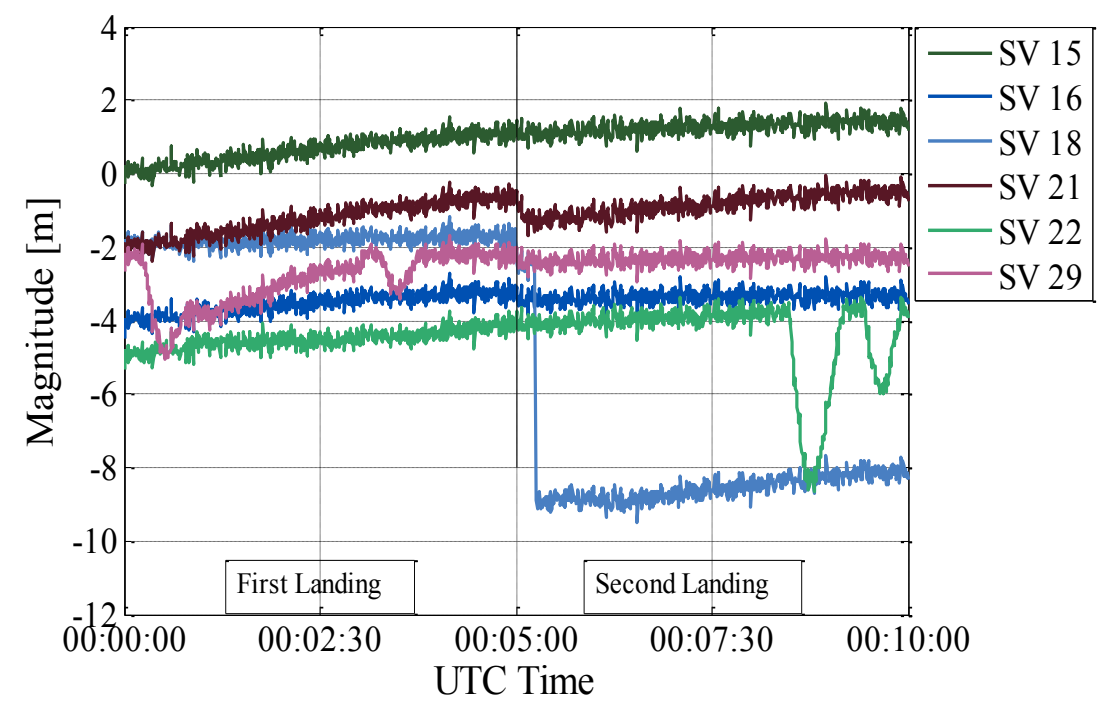

Figure 7.8: Time series of the effects due to ionospheric and tropospheric delays, multipath, phase ambiguity, phase scintillation and random errors on the carrier phase, generated for TAM/JJ3839 flight from 00:00:00 UTC and 00:10:00 UTC on 20 December 2014.

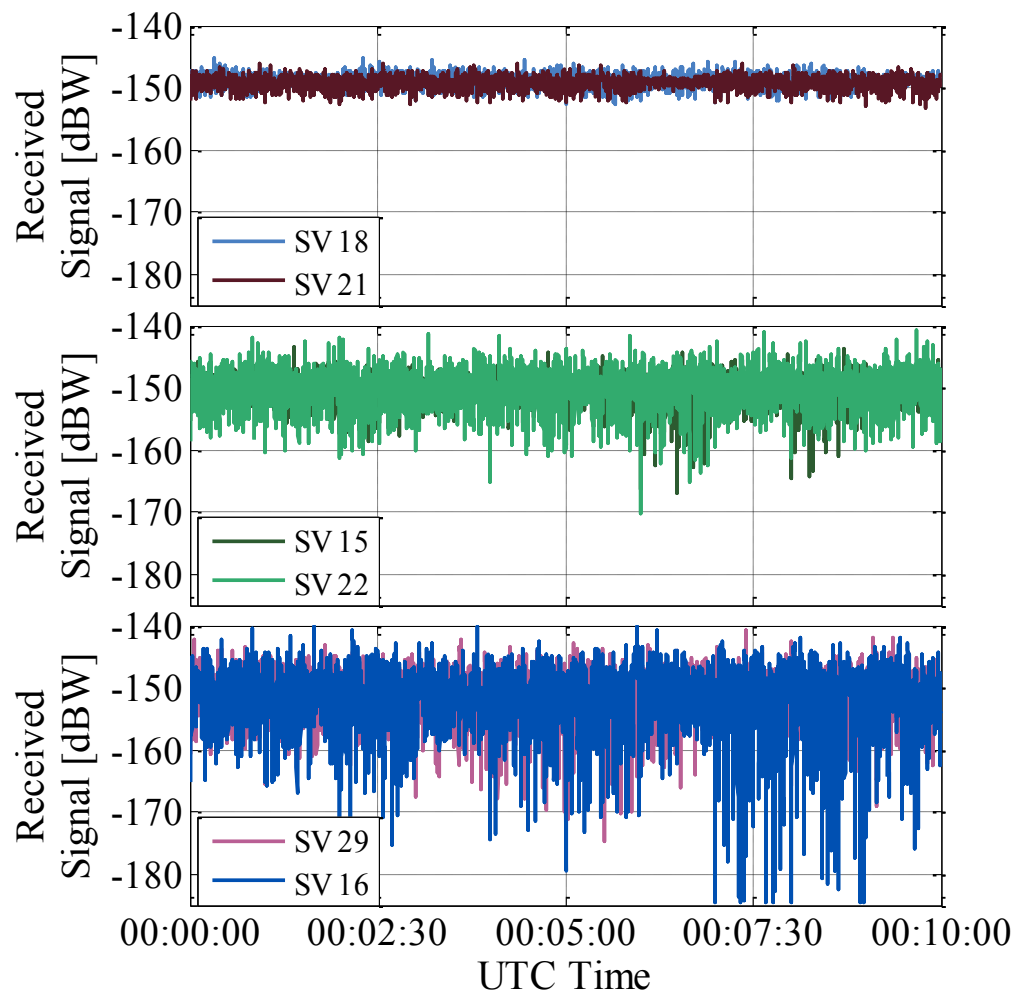

Figure 7.9: Time series of the received signal power, generated for TAM/JJ3839 flight from 00:00:00 UTC and 00:10:00 UTC on 20 December 2014, considering the effects due to amplitude scintillation, multipath, and random errors. 
The magnitude of the combined effects on the pseudorange during the selected period of high solar activity reaches the absolute maximum of $15.84 \mathrm{~m}$ for the first landing, as shown by Figure 7.7. On the other hand, this maximum value for the second landing is approximately $15.54 \mathrm{~m}$. These maximum delays are associated with SV 16 and SV 22, respectively. Indeed, this satellite presented higher delays than the others, due to its lower elevation angles (approximately $29^{\circ}$ ) from the aircraft position, as depicted by skyplot in Figure 7.4. Also, SV 15 presents a lower elevation angle (approximately $29^{\circ}$ ), but the time delay of propagation is less than the SV 16 due to the least ionospheric delay. The minimum delay in the high solar activity day for this scenario, observed in association with SV 18, is 6.14 $\mathrm{m}$, due to the high value of the corresponding elevation angle (approximately $80^{\circ}$ ). Typically, SV 16 presents 2 times the delay of SV 18. For short periods of time, it can vary depend of the presence of ionospheric gradients.

It is important to remember that ionospheric spatial gradients estimated between RIOD and ONRJ stations were added to the ionospheric delay for each satellite. For example, SV 29 and SV 22 present large gradients that reach 220 $\mathrm{mm} / \mathrm{km}$ and $604.5 \mathrm{~mm} / \mathrm{km}$, as depicted in Figure 7.7 and 7.8, respectively. In Figure 7.8 , it is possible to observe the negative values of the combined effects on the carrier-phase observables, due to the advancement provided by the ionospheric delay term, represented by the negative signal in equation (5.2). The large ionospheric delay and ionospheric gradients in SV 16, SV 22 and SV 29 are due the position of their corresponding IPPs in relation to the EIA where large concentrations of electrons (TEC) are estimated as is shown in Figure 7.10. Note that the red line depicts the geomagnetic equator, and orange dashed lines represent the $-8^{\circ}$ and $-20^{\circ}$ geomagnetic latitudes. In addition, large cycle slip is present in the curve corresponding to SV 18. 


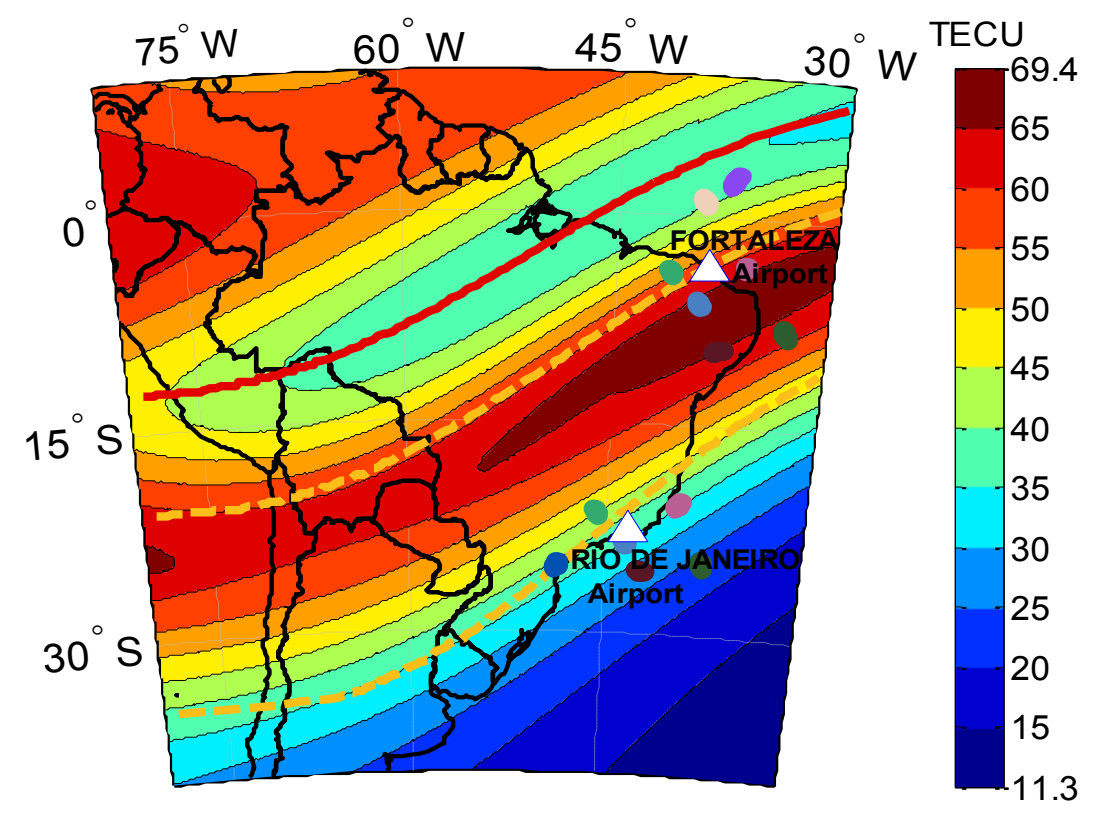

Figure 7.10: IRI+Residual TEC Map and ground projections of the corresponding IPPs of each satellite at 00:10:00 UTC on 20 December 2014.

Each curve in Figure 7.9 displays the results from a simulation designed to generate 10-min time series of received signal power for the six tracked GPS satellites. Amplitude scintillation is one of the ionospheric effects of greater variability in the received signal model. The 3 panels of Figure 7.7 clearly show that the magnitude of deep fades are different for the six GPS satellites, in response to the $\mathrm{S}_{4}$ values indicated in Figure 7.5. That is, the times series of received signal for satellites SV 15, SV 16, SV 18, SV 21, SV 22 and SV 29 can reach values as low as $-168.20 \mathrm{dBW} ;-184.80 \mathrm{dBW} ;-153.70 \mathrm{dBW} ;-153.30 \mathrm{dBW} ;-170.10 \mathrm{dBW}$ and $-175.20 \mathrm{dBW}$, respectively. The minimum received signal for GPS L1 is -158.5 dBW (SPS, 2018), if one satellite presents a received signal power below to the threshold, the corresponding channel is not available. In the same way, the time series of phase scintillation generated for each channel are shown in Figure 7.11. 


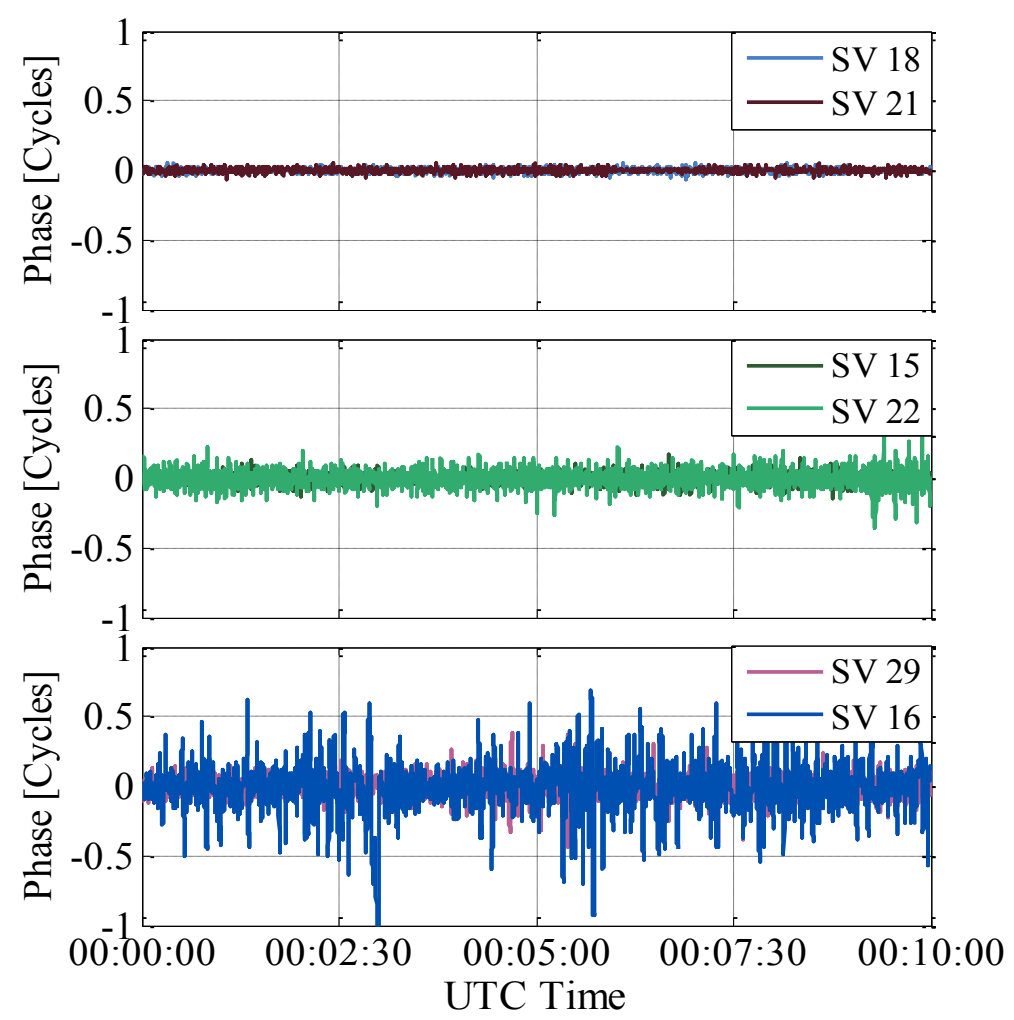

Figure 7.11: Time series of the phase scintillation, generated for TAM/JJ3839 flight from 00:00:00 UTC and 00:10:00 UTC on 20 December 2014.

Figure 7.11 depicts the times series of phase scintillation simulated according to the $\mathrm{S}_{4}$ and the standard deviation of phase scintillation $\sigma_{\varphi .}$ SV16, V 22 and SV 29 present a large fluctuation in comparison to the SV 15, SV 18 and SV 21.

\section{Aircraft positioning errors based on pseudorange observables}

The aircraft positions in ECEF coordinates $\left(x_{j}(k), y_{j}(k), z_{j}(k)\right)$ are estimated using the generated pseudorange observables from channels with received signal power above the reception threshold for each epoch, according the formulation presented in section 6.17. After that, the residual values between the estimated position based on GPS observables and the reference position along the flight path provides the aircraft positioning errors. These errors are estimated using equation (6.75). It is important to note that the estimated errors are due to associated effects (ionospheric and tropospheric delays, ionospheric scintillation, multipath and random errors). 
Many tracking and navigation applications adopt local coordinates North, East and Down (NED) to represent state positions. The results of the position errors expressed in NED coordinates are shown in the left plot of Figure 7.11. Also, horizontal and vertical positioning errors (HPE and VPE) are represented in the right plot of the same Figure, since they are more intuitive and practical for navigation applications than ECEF and Geodetic coordinates. HPE and VPE are estimated applying equations (6.78) and (6.79) to errors expressed in ECEF coordinates, respectively.
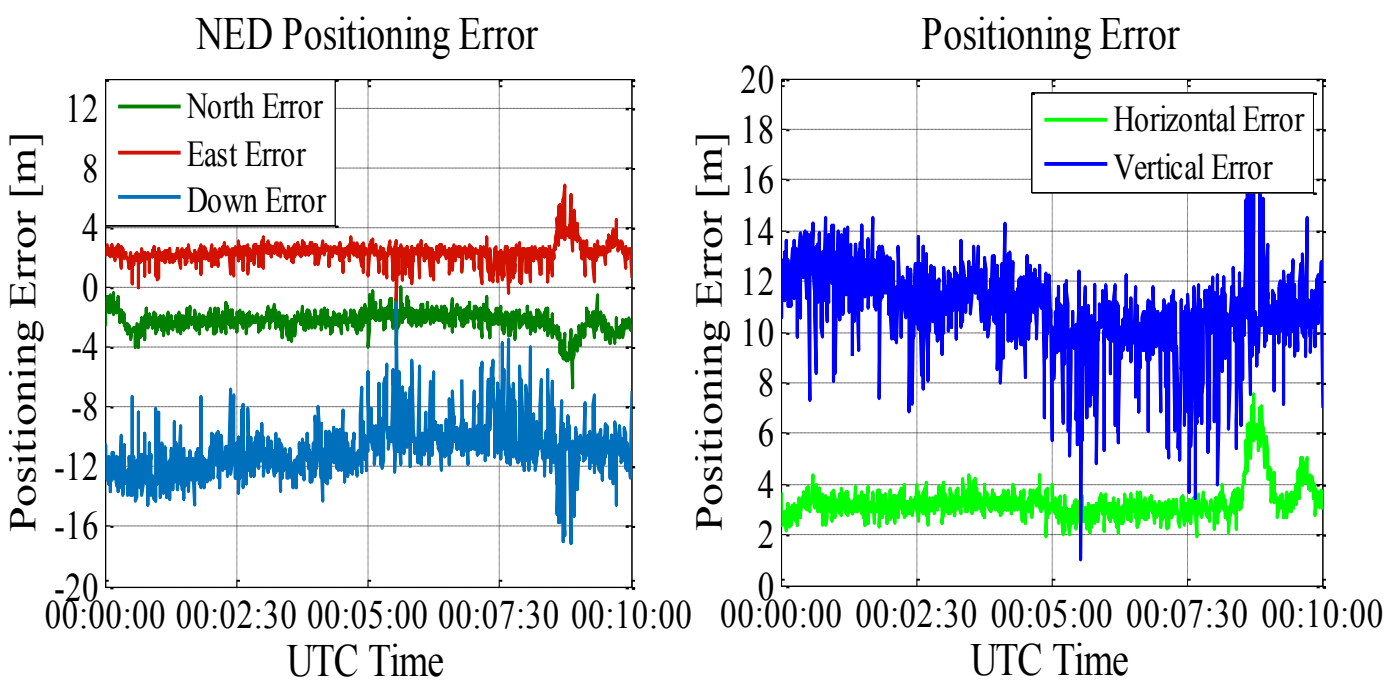

Figure 7.12: NED positioning errors (Left); Horizontal and Vertical positioning errors (Right); all based on pseudoranges generated during TAM/JJ3839 flight from 00:00:00 UTC to 00:10:00 UTC on 20 December 2014

Both plots of Figure 7.12 show that the down/vertical coordinate presents large errors in comparison with those associated with the east, north and horizontal coordinates, due to the listed effects and to the user-satellite geometry. The right plot of Figure 7.12 reveals Vertical Position Errors that exceed the Vertical Alert Limit (VAL) of $10 \mathrm{~m}$ for CAT-I during most of the time interval, reaching the maximum value of $17.5 \mathrm{~m}$.

The aim of a GBAS is to reduce the positioning errors due to the ionospher ic, tropospheric effects and random errors using differential corrections. The next step is to test the signal in space and generate pseudorange corrections. 


\section{GBAS monitor results}

The aircraft positioning error will be estimated with basis on corrected pseudoranges (combining direct GPS observables and differential corrections estimated from GBAS), using the point positioning method. Thus, time series of pseudorange $P R_{(i, j)}(k)$, carrier-phase $\phi_{(i, j)}(k)$ and received signal power $C_{(i, j)}(k)$ are generated for all the combinations of six tracked GPS satellites $(i=\mathrm{SV} 15, \mathrm{SV}$ 16, SV 18, SV 21, SV 22 and SV 29) and GBAS receivers located at the Rio de Janeiro Airport ( $j=$ RSMU1, RSMU2, RSMU3 and RSMU4) from 00:00:00 UTC and 00:10:00 UTC on 20 December 2014, with time denoted by $(k)$.

The generated GPS observables are injected into the Integrity Monitor Testbed (IMT) of the GBAS Ground Facility, as shown in Figure 6.1. As previously discussed, the main function of this monitor is to detect a varied array of possible anomalies or failures in the signal in space and generation of differential corrections for each epoch. The differential corrections are estimated using pseudorange and carrier-phase observables of channels $(i, j)$ that not presents large signal fadings or phase deviations induced by ionospheric scintillation. Additionally, it is necessary to identify and isolate potential anomalies and failures in the Signal in Space, through the tests performed by Quality Monitors described in Chapter 6. Only the observables and the corresponding received signal power of each satellite that pass the tests are used to generate time series of differential corrections for pseudorange observable from each available channel. The results of the applied tests and differential corrections are shown in the next paragraphs.

A variety of algorithms are grouped into the Signal Quality Monitor (SQM), Data Quality Monitor (DQM), Measurement Quality Monitor (MQM), Multiple Reference Consistency Check (MRCC) and Message Field Range Test (MFRT). The purpose of these monitors is detect a wide range of possible failures in the GPS Signal in Space or in the GBAS Ground Facility model itself. Each monitor is explained in detail in Chapter 6. Results for the RSMU1 station are presented in this section, Recall that the same procedure is repeated for each GBAS receiver. 
The Measurement Quality Monitor (MQM) detects step and impulsive errors in the signal in space. MQM includes three monitors that are combined into a single flag ' $0 / 1$ ' by the logical 'or' operator to indicate if the corresponding channel passed the tests or not. The carrier step and acceleration tests were applied to each available channel between GPS satellites tracked by GBAS receivers. The $\operatorname{Acceleration}_{(i, j)}(k)$ and $\operatorname{Step}_{(i, j)}(k)$ time series estimated for each available channel (between $i=\mathrm{SV} 15, \mathrm{SV} 16, \mathrm{SV} 18, \mathrm{SV} 21, \mathrm{SV} 22$, SV 29 and $j=\mathrm{RSMU1}$ ) from 00:00:00 UTC to 00:10:00 UTC on 20 December 2014 are shown in Figures 7.13 and 7.14, using the same color code of the previous plots and red lines to indicate the respective threshold.

The results of the carrier acceleration and carrier step tests in Figures 7.13 and 7.14 are used to validate (or not) the consistency of carrier phase measureme nts over the epochs to detect rapid changes due to phase scintillation and satellite clock anomalies that could cause errors in the pseudorange corrections. Phase scintillation is directly affected by ionospheric irregularities and intense events of this phenomenon increases the probability of signal loss of lock, as described by Forte (2007) and Moraes et al. (2017).

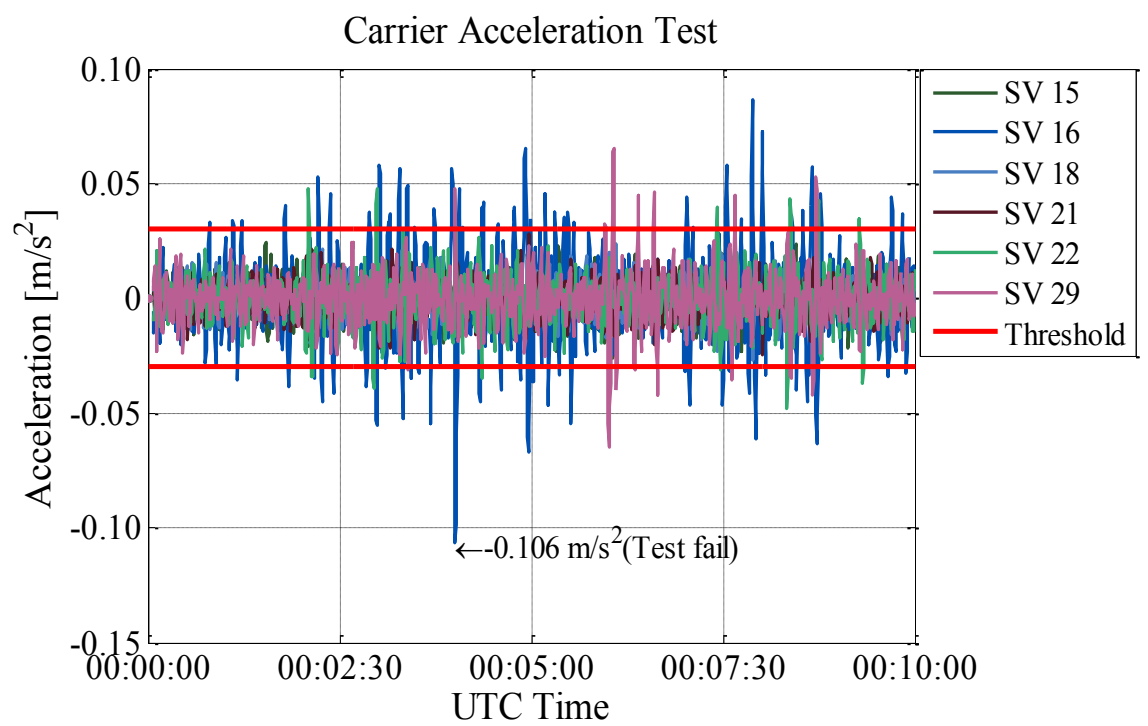

Figure 7.13: MQM Carrier Acceleration test results for the RSMU1 GBAS receiver during TAM/JJ3839 flight from 00:00:00 UTC to 00:10:00 UTC on 20 December 2014. 


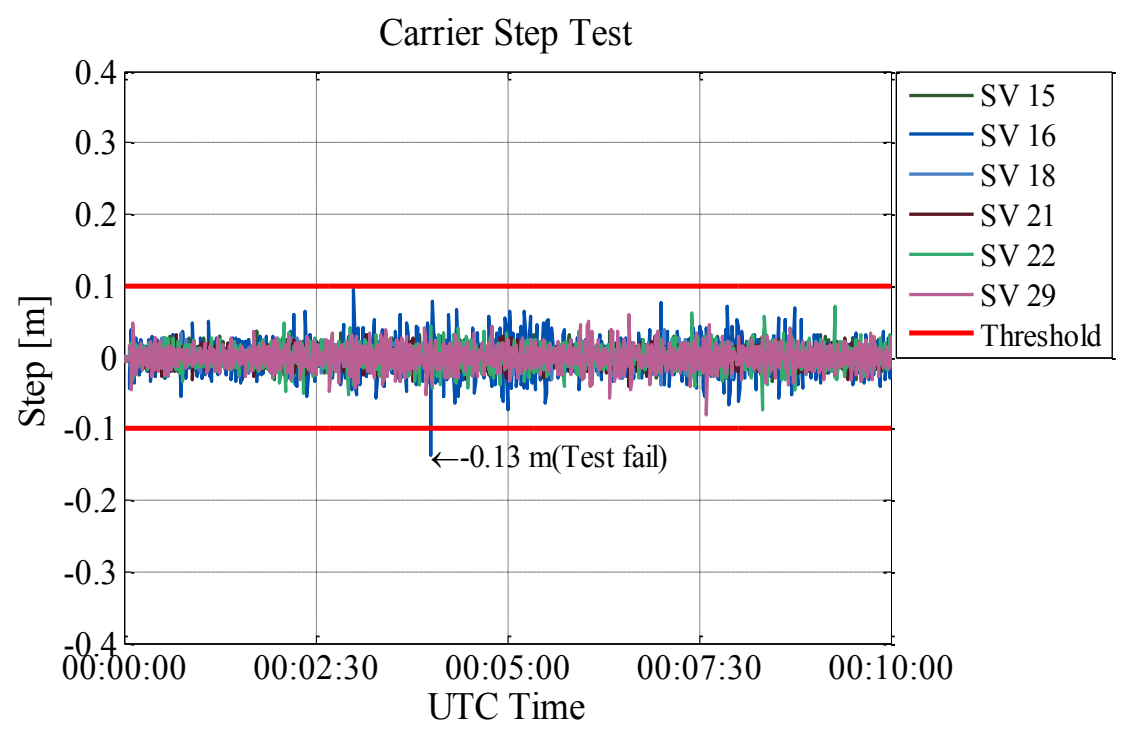

Figure 7.14: MQM Carrier Step test results for the RSMU1 GBAS receiver during TAM/JJ3839 flight from 00:00:00 UTC to 00:10:00 UTC on 20 December 2014.

In the current case, most channels passed both tests during the time interval,

since the estimated results are within the respective thresholds $E_{\text {th_acce }}=2.6 \mathrm{~cm} / \mathrm{s}^{2}$ and $E_{\text {th_step }}=0.1 \mathrm{~m}$. The most notable exception is the SV 16 channel, which presented an acceleration of $|-0.106| \mathrm{cm} / \mathrm{s}^{2}$ and step of $0.13 \mathrm{~m}$, values that exceed the corresponding threshold (and consequently not pass the tests). SV 16 presents a large acceleration many epochs. These results are consistent with the rapid phase changes presented by the time series of phase scintillation generated for each channel, shown in Figure 7.11. On the other hand, SV 18, SV 22, and SV 29 channels present moderate to strong phase scintillation characterized by the $\sigma_{\varphi}$ index. It is important to note that the performance of the carrier smoothing algorithms could be affected by the presence of moderate to strong phase scintillation.

The innovation test used the carrier-phase times series to smooth the pseudorange observable. The associated algorithms decrease random errors, considering that the pseudorange observable is noisier than the carrier phase observable. Figure 7.15 shows that pseudoranges $P R_{(i, j)}(k)$ (between $i=\mathrm{SV} 15$, SV 16, SV 18, SV 21, SV 22, SV 29 and $j=$ RSMU1) passed the test $\operatorname{Inno}_{(i, j)}(k)$ for all available channels from 00:00:00 UTC and 00:10:00 UTC on 20 December 2014. However, SV 16, SV 22 and SV 29 channels present relatively large values within the threshold, due the magnitude of the associated delay effects that can be 
observed in Figure 7.7. The large jump of $1.4 \mathrm{~m}$ in the estimated values for channel SV 16 is produced by a filter reset operation.

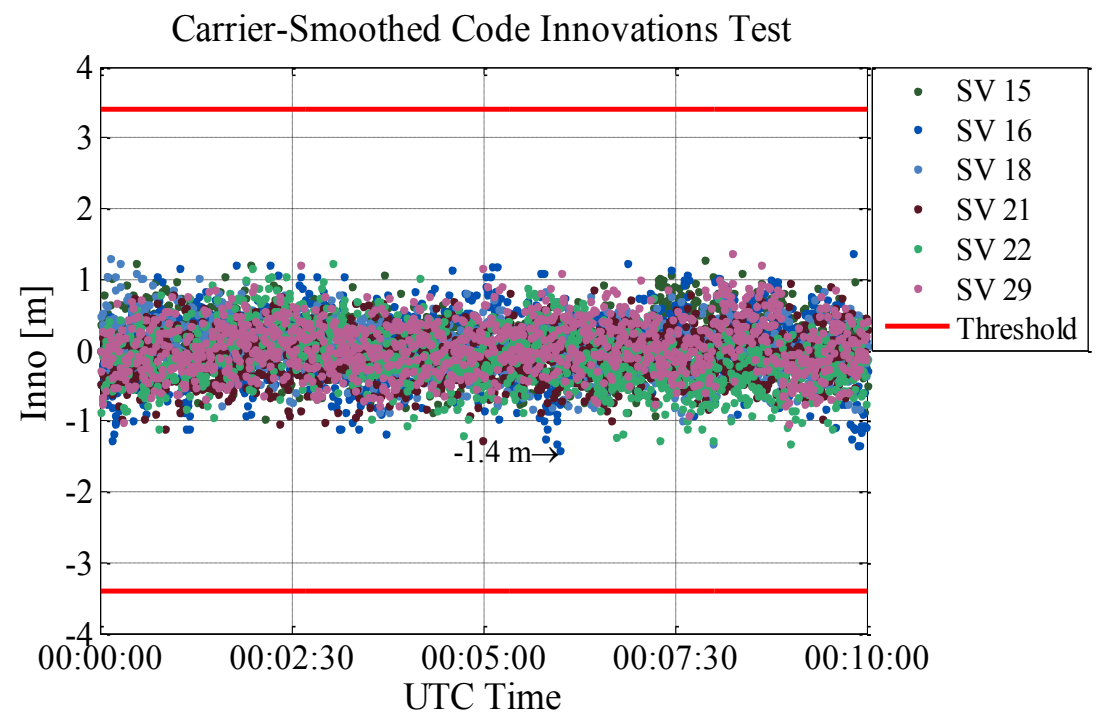

Figure 7.15: MQM Carrier-Smoothed Code Innovations test results for the RSMU1 GBAS receiver during TAM/JJ3839 flight from 00:00:00 UTC to 00:10:00 UTC on 20 December 2014.

The Signal Quality Monitor (SQM) detects and identifies anomalies in the received GPS signals. SQM includes three monitors: the correlation peak symmetry, the received signal power, and code-carrier divergence tests, that are combined into a single flag ' $1 / 0$ ' (by logical the 'or' operator).

The correlation peak symmetry test was applied to each available channel. Therefore, $D_{s(i, j)}(k)$ values were estimated using equation (6.14). The estimated results can be observed in Figure 7.16, using the same color code of the previous plots and red lines to indicate the respective threshold. The correlation peak symmetry test was implemented in the SQM to detect GPS multipath distortions by incorporating correlators. To evaluate multipath effects, two correlator spacings equal to 1 chip and 0.5 chip, where $\mathrm{T}_{\mathrm{N}}=0.5 \mathrm{~T}_{\mathrm{C} / \mathrm{A}}$. Recall that multipath and its effects were described in Chapter 5 considering different chip widths. In Figure 7.15, it is possible to observe that the values obtained for $D_{s(i, j)}(k)$ are within the threshold $\left(E_{\text {th_co }}=0.3 \mathrm{~m}\right)$, for all actives channel. 


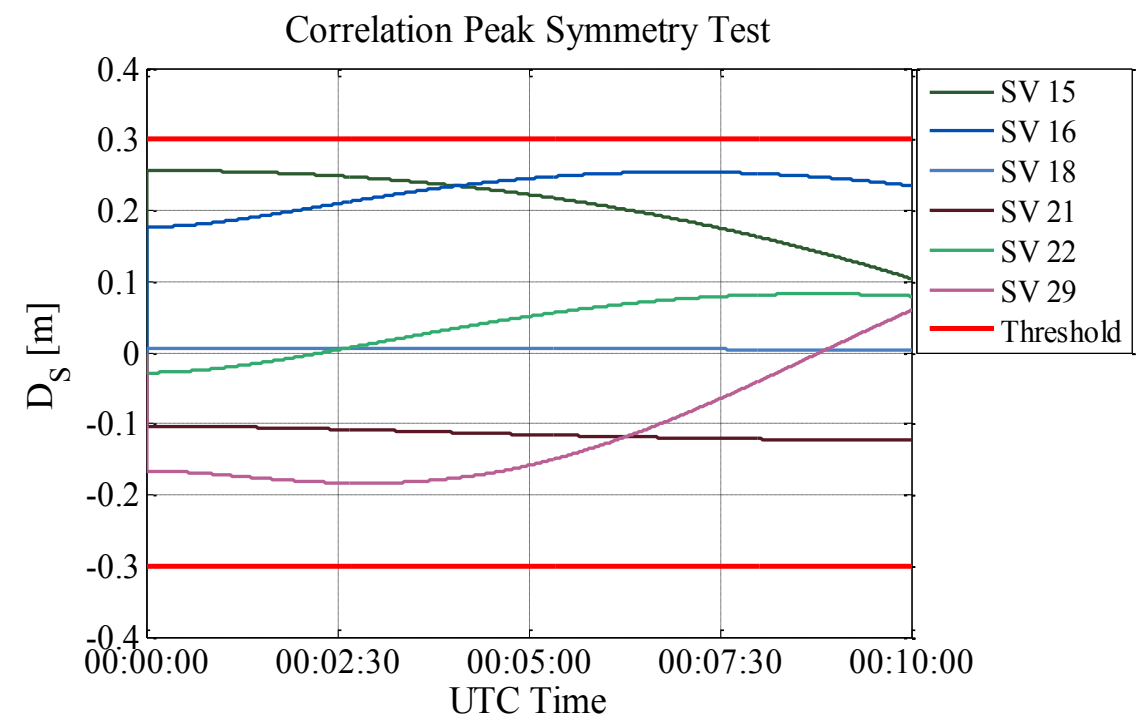

Figure 7.16: SQM Correlation Peak Symmetry test results for the RSMU1 GBAS receiver during TAM/JJ3839 flight from 00:00:00 UTC to 00:10:00 UTC on 20

December 2014.

The largest observed values $(0.26 \mathrm{~m}$ and $0.25 \mathrm{~m})$ are obtained for SV 15 and SV 16, respectively. These results are coherent with the geometry and environment, since these satellites display low elevation angles with respect to the RSMU1 receiver. Therefore, increased multipath effects are expected, as indicated by the skyplot in Figure 7.4.

The code-carrier divergence test facilitates the interoperability of receivers from different manufacturers on the ground and in the air. It is also used to detect temporal ionospheric gradients. This test was applied to each available channel between GPS satellites tracked by GBAS receivers. Figure 7.17 shows the results of $D v g c_{(i, j)}(k)$ estimated for each channel at the RMSU1 receiver, represented by color dots (using the same color code of the previous plots) and indicating the threshold by red dots. Figure 7.17 shows that the six channels passed the test: indeed, the corresponding results are within the area limited by the threshold, defined as $0.015 \mathrm{~m} / \mathrm{s}$. The maximum observed value is $0.0097 \mathrm{~m} / \mathrm{s}$, which occurs for the channel SV 29 - RSMU1. It is relevant to mention, GPS satellites that presents large magnitude of effects associated in the pseudorange observable (ionospheric and tropospheric delays, multipath and random errors), also present large values of $\operatorname{Dvgc}_{(i, j)}(k)$, as is show for the channel of SV29, SV22 and SV16. 


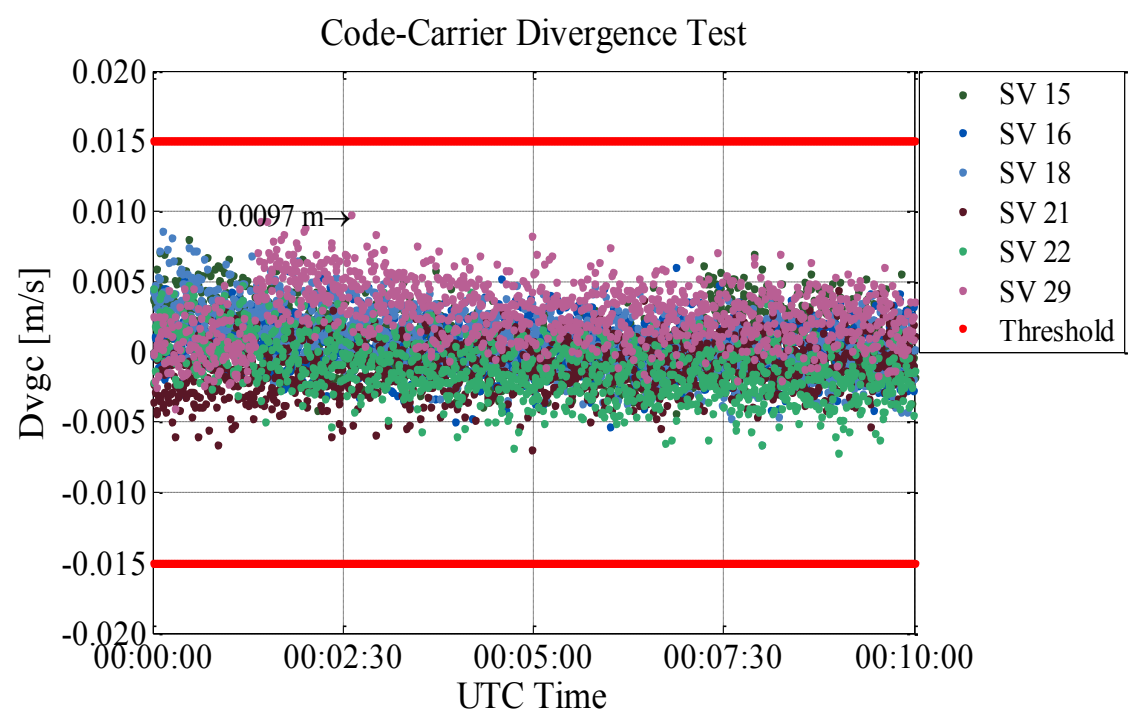

Figure 7.17: SQM Code-Carrier Divergence test results for the RSMU1 GBAS receiver during TAM/JJ3839 flight from 00:00:00 UTC to 00:10:00 UTC on 20

December 2014.

The received signal power test is the third applied to each available channel between GPS satellites tracked by GBAS receivers within the MQM monitor. Figure 7.18 shows the time series of received signal power to noise density ratio $\left(C / N_{0}\right)_{(i, j)}(k)$ estimated for all GPS satellites tracked by the RSMU1 receiver between 00:00:00 and 00:10:00 UTC on 20 December 2014, using the same color code of the previous plots and red lines to indicate the respective lower threshold. The received signal power to noise density ratio estimated for SV 15, SV 18, and SV 21 are above the threshold value $E_{t h_{-} R S P}=35 \mathrm{dBHz}$ during all the time interval. On the other hand, SV 16, SV 22. and SV 29 channels display deep fadings that take the ratio $C / N_{0}$ to values below the reception threshold at some epochs, as shown by the lower plot of Figure 7.18. Therefore, these satellites do not pass the corresponding test at the respective epoch. SV 16 is the most affected channel, which presents a lower value of $C / N_{0}=24.5 \mathrm{dBHz}$, associated with a deep signal fading due to amplitude ionospheric scintillation characterized by the $\mathrm{S}_{4}$ index observed in Figure 7.5. 


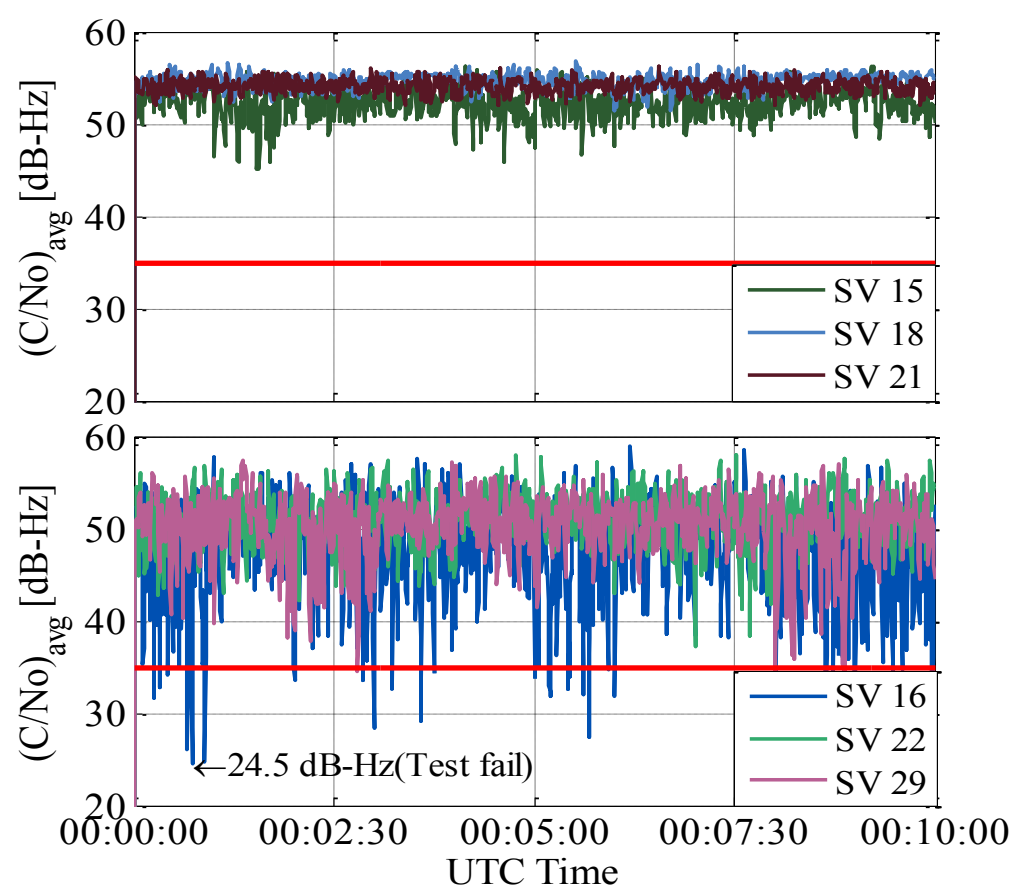

Figure 7.18: SQM Received Signal Power test results for the RSMU1 GBAS receiver during TAM/JJ3839 flight from 00:00:00 UTC to 00:10:00 UTC on 20

December 2014.

It is important to recall that the ionospheric scintillation degrades the signal propagation and induces the unavailability of service in receivers of the Global Positioning System. This scintillation affects irregularly according temporal variation, seasonal variation, observation locations, geophysical conditions. As mentioned in the Chapter $5, \alpha-\mu$ statistical distributions are used to model the scintillation amplitude based on location distributed in different geomagnetic latitudes. The aim of this work is try to analyze different scenarios and examine the functionality of the GBAS system in these scenarios.

The Data Quality Monitor (DQM) verifies that the navigation data present sufficient fidelity. DQM includes two test: Yesterday Ephemerides Minus Today Ephemerides test and Data Parity test, that are combined into a single flag ' $1 / 0$ ' (by the logical 'or' operator). The Yesterday Ephemeris-Today Ephemeris test compares the ECEF satellite position based on the received new and old ephemeris message, validated over the past 24 hours. This test was executed to each available channel. Figure 7.19 illustrates the residual value estimated by the differences between the coordinates of the satellite positions $x_{i}(k), y_{i}(k)$, and $z_{i}(k)$ obtained 
from the ephemerides received on 20 December 2014 at the Rio de Janeiro Station and those corresponding to a time-of-ephemeris (toe) 24 hours earlier.

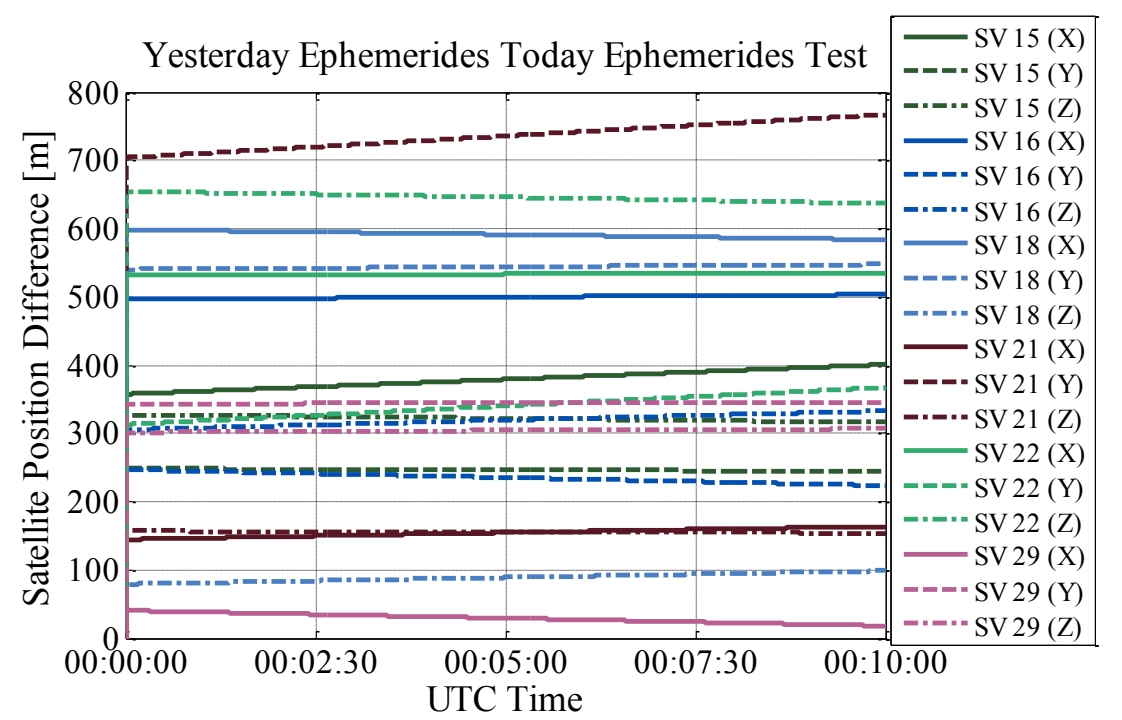

Figure 7.19: DQM Yesterday Ephemerides minus Today Ephemerides (Ye-Te)

test results for the RSMU1 GBAS receiver during TAM/JJ3839 flight from 00:00:00 UTC to 00:10:00 UTC on 20 December 2014.

The position differences in ECEF coordinates shown in Figure 7.19 are due error in satellite clocks or small variation in Keplerian elements that take place during the period from toe(YE) to toe(TE), since it is known that nominal ephemeris messages are very accurate within their fitting intervals. The magnitude of differences is located in the interval $(18 \mathrm{~m}, 766 \mathrm{~m})$. The threshold for this test is $7000 \mathrm{~m}$. Since the differences are within this threshold, these ephemerides are validated. These differences can be transformed into crosstrack, intrack and radial components, as described by Pullen (2001).

The data parity test confirms that the IODE and IODC messages of navigation files match. This test compares information that do not allow graphical representations. For this reason, it will not be further discussed.

According to the methodology described in Chapter 6, all the QM results are analyzed by the EXM monitor, where the matrix $\mathrm{Q}(k)$ is constructed for each channel $(i, j)(i=\mathrm{SV} 15, \mathrm{SV} 16, \mathrm{SV} 18, \mathrm{SV} 21, \mathrm{SV} 22, \mathrm{SV} 29$ and $j=\mathrm{RSMU} 1$, RSMU2, RSMU3 and RSMU4) with basis on the test results. Next, the decision 
matrix $\mathrm{D}(k)$ is conformed using the logical 'or' operation based on the matrices $\mathrm{Q}(k)$. Then, the 'or' operation is applied to the tracking matrix $\mathrm{T}(k)$ and decision $\mathrm{D}(k)$, yielding the $\mathrm{EXM}$ matrix $\mathrm{ED}(k)$. The isolation process separates the unavailable GPS satellites from the process of correction generation. As an example, Figure 7.20 shows the satellites that have been isolated after analysis of the EXM monitor for the RSMU1 receiver.

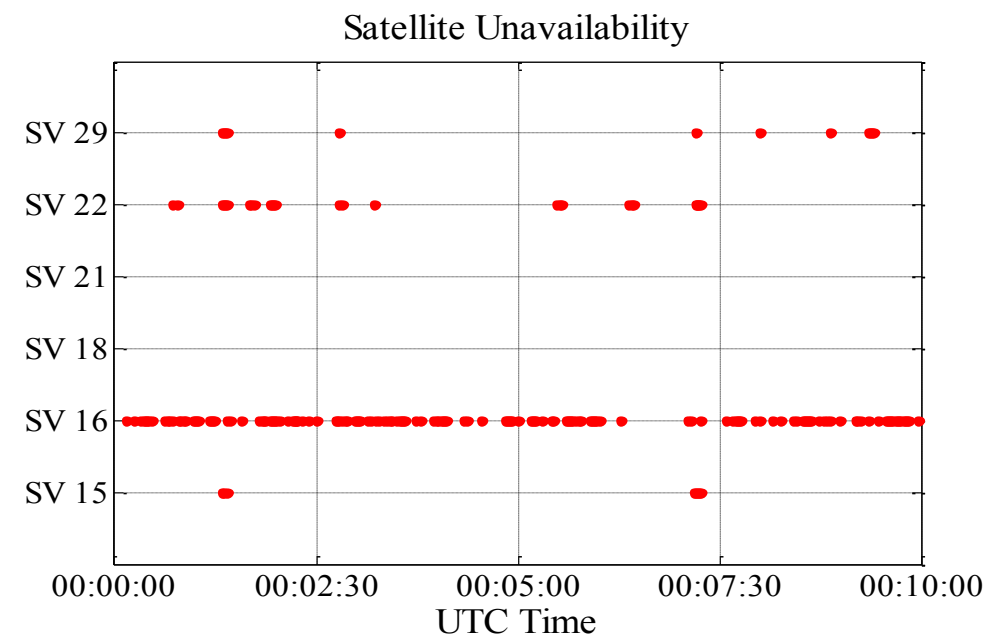

Figure 7.20: Unavailable GPS satellites for the RSMU1 GBAS receiver during TAM/JJ3839 flight from 00:00:00 UTC to 00:10:00 UTC on 20 December 2014.

Figure 7.20 indicates that the EXM monitor isolated SV 16 during many epochs. Reviewing the results of the QM tests, it is possible to define that SV 16 was unavailable because it presented deep signal fading and phase acceleration occasioned by scintillation due to the GPS signal propagation through the equatorial ionospheric anomaly crest. This analysis is consistent with the work by de Paula et al. (2003), who reported that strong scintillation with $\mathrm{S}_{4}$ exceeding 0.5 has only been observed under the EIA crest.

The Multiple Receiver Consistency Check (MRCC) expresses the consistency of the corrections produced for each satellite across all reference stations. To accomplish this task, this test computes the B-values $B_{P R(n, m)}(k)$ using equations (6.38). B-values are broadcast by the ground system for the aircraft subsystem to determine the number of reference receivers that produce valid pseudorange measurements for a given ranging source. The B-values were 
estimated for each available channel. Figure 7.21 shows the estimated B-values for receiver RSMU1 (color dots) and the corresponding threshold (red dots).

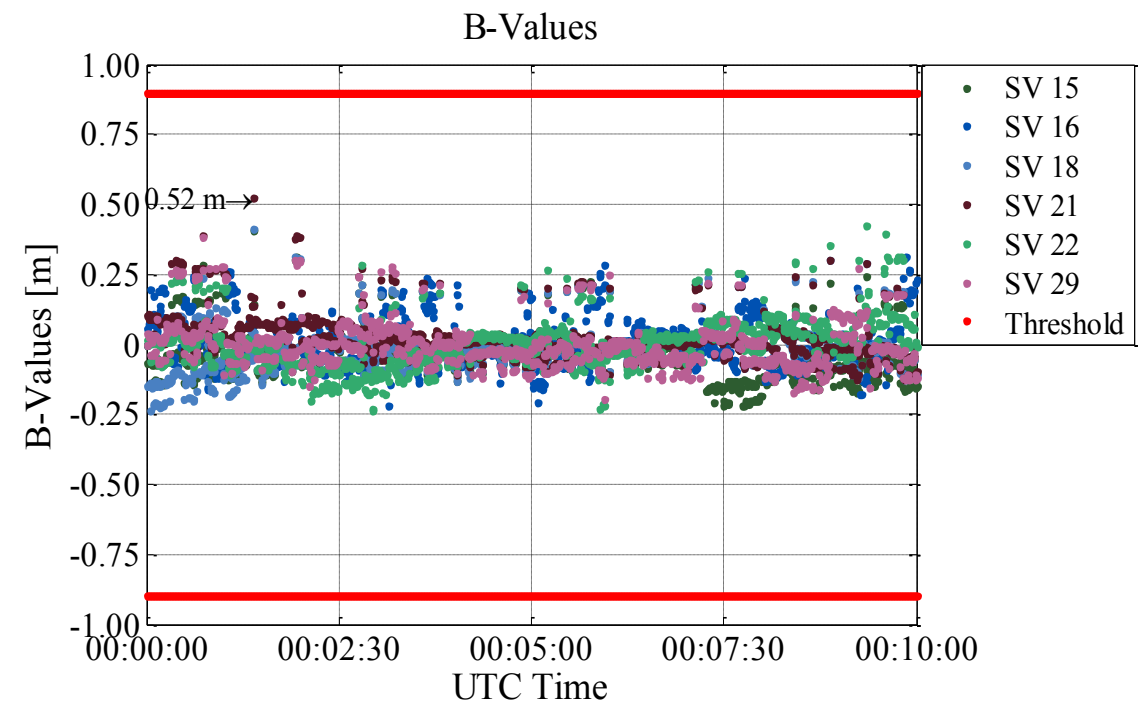

Figure 7.21: MRCC B-values test results for the RSMU1 GBAS receiver during

TAM/JJ3839 flight from 00:00:00 UTC to 00:10:00 UTC on 20 December 2014.

In Figure 7.21, the results of the B-values estimated for SV 15, SV 16, SV 18, SV 21, SV 22 and SV 29 at RSMU1 reveal variations between $|-0.25| \mathrm{m}$ and 0.52 $\mathrm{m}$. For example, SV 21 presents a peak value of $0.52 \mathrm{~m}$. Generally, the peaks occur at epochs of greater magnitude of the differential corrections. All the estimated Bvalues do not exceed the associated threshold.

The Message Field Range Test (MFRT) is the last one performed before corrections are approved for broadcast. It estimates pseudorange corrections rates $R_{P R_{\text {corr }(n)}}(k)$ for each validated satellite $n$, using equation (6.46). It ensures that the correction rates are less that the threshold $(3.4 \mathrm{~m} / \mathrm{s})$. Figure 7.22 depicts the values of $R_{P R_{\text {corr }(n)}}(k)$ estimated for ( $n=\mathrm{SV} 15, \mathrm{SV} 16, \mathrm{SV} 18, \mathrm{SV} 21, \mathrm{SV} 22$ and SV 29) (color lines), bounded by the threshold (red line). 


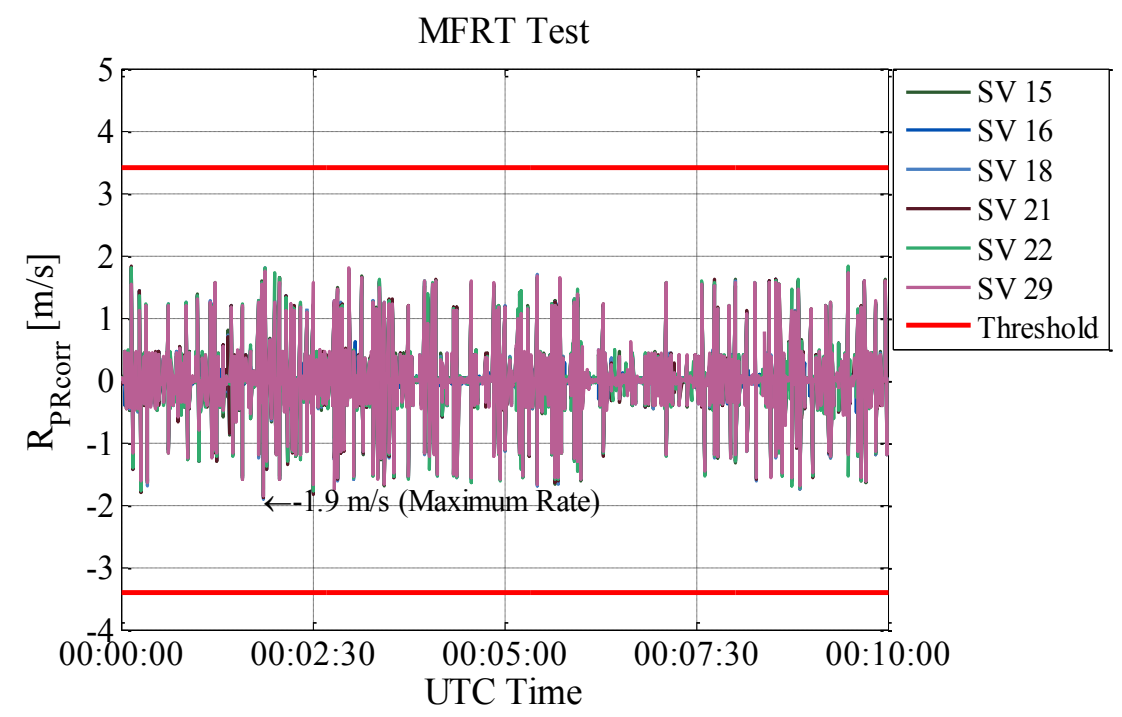

Figure 7.22: MFRT test result for the GBAS system during TAM/JJ3839 flight from 00:00:00 UTC to 00:10:00 UTC on 20 December 2014.

It is possible to analyze that the values of $R_{P R_{\text {corr }(n)}}(k)$ estimated for each satellite present peak variations between $|-1.90|$ and $1.85 \mathrm{~m} / \mathrm{s}$, as shows Figure 7.22. However, most variations are closer to $0 \mathrm{~m} / \mathrm{s}$. SV 29 presents a peak value of $|-1.90|$ $\mathrm{m} / \mathrm{s}$. All the pseudorange correction rates estimated for available channels do not exceed the threshold. Consequently, all the corrections from available channels are approved and transmitted to the aircraft.

\section{Pseudorange corrections}

One of the main goals of the present work is the estimation of the differential correction $P R_{\operatorname{corr}(n)}(k)$ for each tracked and validated GPS satellite $n$. The pseudorange corrections are computed using equations (6.36), that depend on the smooth and clock adjustment processes. The smoothing of the pseudorange using the carrier phase observable is relevant to this process. The pseudorange observables (color lines) and smoothed pseudoranges (red lines) for channels $(i, j)$ ( $i=\mathrm{SV} 15, \mathrm{SV} 16, \mathrm{SV} 18, \mathrm{SV} 21, \mathrm{SV} 22, \mathrm{SV} 29$ and $j=\mathrm{RSMU} 1)$ for the 10-minute period under discussion are shown in Figure 7.23, after the subtraction of the respective contributions of the geometric distance and satellite clock error. 


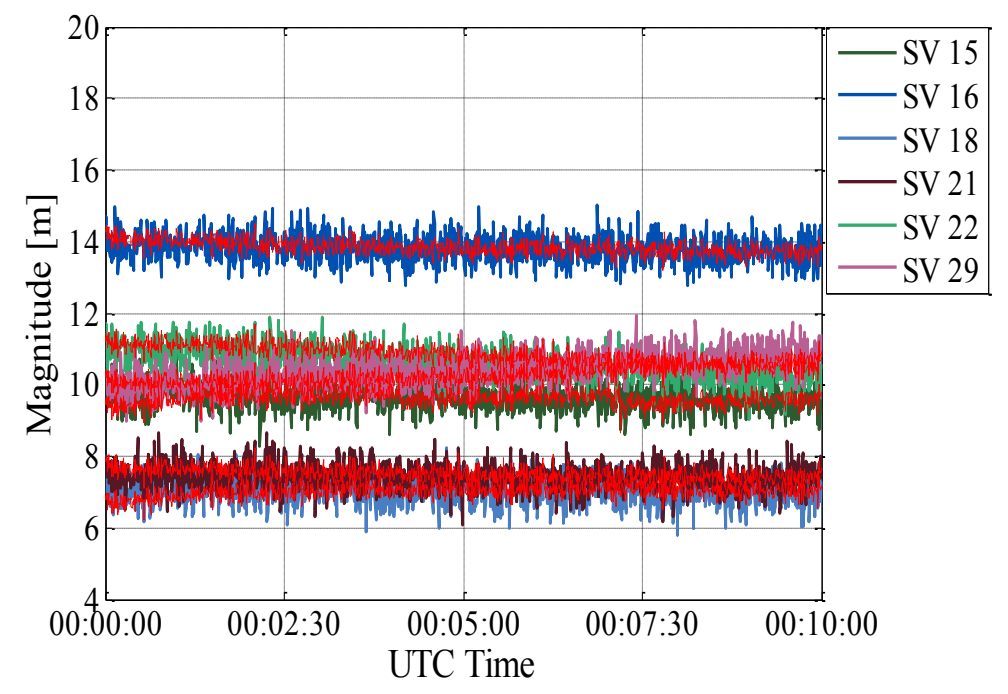

Figure 7.23: Time series of the pseudoranges (color) and smoothed pseudoranges (red), excluding the respective geometric distances and satellite clock errors, generated for TAM/JJ3839 flight from 00:00:00 UTC to 00:10:00 UTC on 20 December 2014.

The estimated $P R_{\operatorname{corr}(n)}(k)$ results for $n=\mathrm{SV} 15, \mathrm{SV} 16, \mathrm{SV} 18, \mathrm{SV} 21, \mathrm{SV}$ 22 and SV 29 are illustrated in Figure 7.24. Recall that the indices $(n, m)$ are adopted instead of indices $(i, j)$, to indicate validated channels after the isolation procedures.

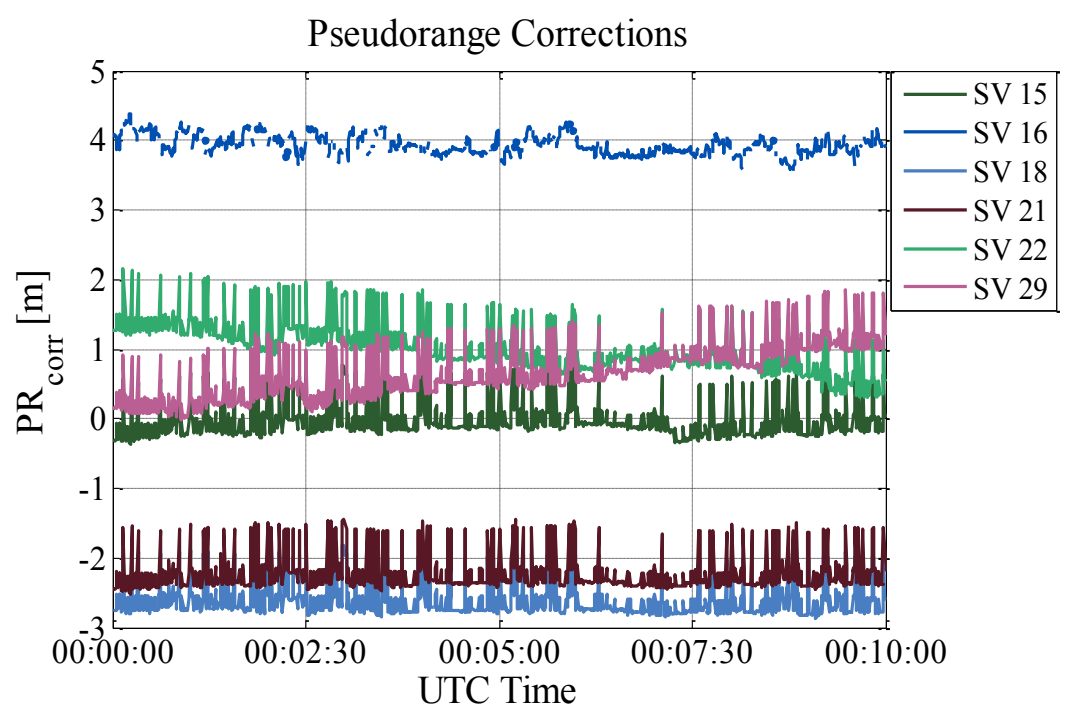

Figure 7.24: Pseudorange corrections estimated by the GBAS during TAM/JJ3839 flight from 00:00:00 UTC to 00:10:00 UTC on 20 December 2014.

The large correction values are registered for satellites that are closer to the horizon, with low elevation angle (considering that a $20^{\circ}$ satellite elevation mask 
was used). For example, the magnitude of the SV 16 correction reached $4.4 \mathrm{~m}$. The corrections are reduced when satellites ascend toward the zenith direction. The $P R_{\operatorname{corr}(n)}(k)$ results are consistent with the literature (Seeber, 2003; Monico, 2008), which indicates that GNSS observations carried out by satellites close to the horizon generally present a higher occurrence of systematic errors due to the ionosphere, troposphere, multipath, and other sources.

\section{Aircraft position based on corrected pseudoranges using GBAS}

After the process of monitoring and generation of corrections, the GBAS station transmit the differential corrections to the aircraft in GBAS messages through the Very High Frequency Data Broadcast (VDB) subsystem. Then, the aircraft recalculate the pseudorange for each satellite and estimates its corrected position. The estimation of the corrected pseudorange is based on smoothed pseudorange $P R_{\text {smoothed }(i, j)}(k)$ and pseudorange correction $P R_{\operatorname{corr}(i)}(k)$ estimated from GBAS system using equation (6.74) for each available channel $(i$, j) $(i=\mathrm{SV} 15, \mathrm{SV} 16, \mathrm{SV} 18, \mathrm{SV} 21, \mathrm{SV} 22, \mathrm{SV} 29$ and $j=$ aircraft $)$.

To evaluate the aviation navigation performance for approaches using the GBAS at the Rio de Janeiro Airport, this subsection shows the estimated positioning errors of the aircraft approach in NED coordinates in the left panel of Figure 7.25. The positioning errors can also be presented in Horizontal and Vertical components, shown in the right panel of Figure 7.25.

Recall that the 10-min simulation, using statistical data, was subdivided into two 5-min intervals (for the first and second approaches): 1) 00:00:00 UTC to 00:05:00 UTC; 2) 00:05:00 UTC to 00:10:00 UTC. Each landing has 2 phases: APV and CAT-I. The durations of these phases are $3 \mathrm{~min}$ and $2 \mathrm{~min}$, respectively.

After the aircraft applied the GBAS corrections, both plots of Figure 7.25 show that the positioning errors in down/vertical coordinate, as well as the east, north and horizontal coordinates present reduced values at many epochs, in comparison with the errors estimated in the associated coordinates based on uncorrected pseudoranges (Figure 7.12). This favorable result occurs when the 
observables are available and validated for GBAS and aircraft receiver, improving positioning. In the current scenario, the GBAS reduced the positioning errors from uncorrected and corrected positioning for each epoch between 11\% and 98\%, approximately, for example, before and after the correction if a vertical component presents an error of $10.5 \mathrm{~m}$ and $2.9 \mathrm{~m}$, the GBAS reduced the error in $72.4 \%$. On the other hand, the epochs with still large errors agree with those associated with greater numbers of unavailable channels to generate corrections, as shown by Figure 7.20, which depicts the unavailability of SV 16 during most of the period under investigation. It should be remembered that Figure 7.20 only refers to the RSMU1 GBAS receiver and geometry also contributes to the remaining errors.
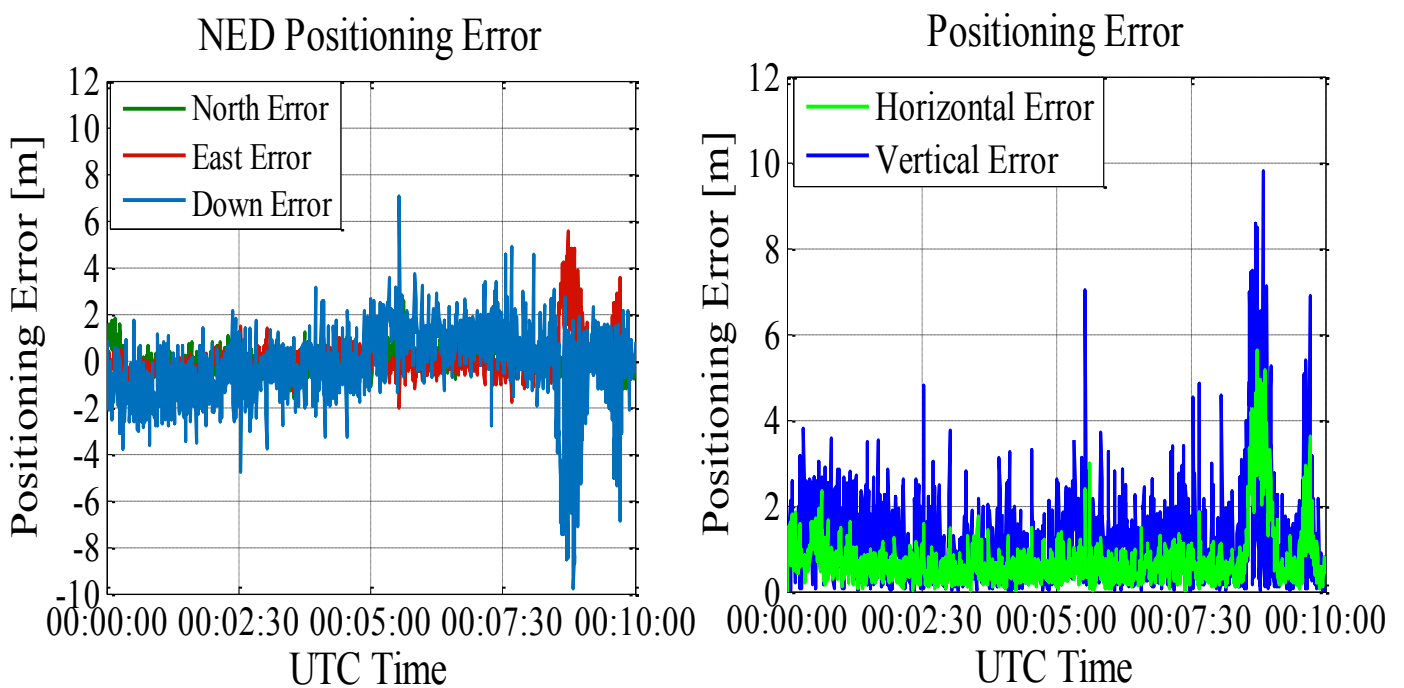

Figure 7.25: NED positioning errors (Left); Horizontal and Vertical positions errors of TAM/JJ3839 flight (Right) based on corrected pseudoranges from 00:00:00 UTC to 00:10:00 UTC on 20 December 2014.

The right plot of Figure 7.25 reveals that vertical position errors (blue line) present peak values due to the reduced number of satellites that generated corrections from the GBAS, reaching the maximum value of $9.8 \mathrm{~m}$ for CAT-I and 7.1 $\mathrm{m}$ for APV. Also, the spatial gradient analyzed between 00:08:30 UTC and 00:10:00 UTC presents larger errors of positioning in the horizontal and vertical component, depicted in a crest form as is shown in both panels of Figure 7.25. The generated spatial gradient $604.5 \mathrm{~mm} / \mathrm{km}$ is considered by Mirus (2015) as a relevant feature in the ionosphere, that degrades the position accuracy. This spatial gradient, due to ionospheric irregularities, are responsible for horizontal and vertical position 
errors equal to $5.7 \mathrm{~m}$ and $9.8 \mathrm{~m}$, respectively. This simulation confirms that ionospheric gradients, which are not directly detected by the GBAS monitor located at the Rio de Janeiro Airport, affects the positioning accuracy.

This case presents results of one scenario with a strong ionospheric impact, represented by spatial gradients and scintillation. For this reason, statistical parameters were estimated to evaluate the accuracy for CAT-I and APV in this unfavorable scenario, as Table 7.6 indicates. Recall that the error estimates based on uncorrected pseudoranges are due to ionospheric and tropospheric delays, multipath and random errors.

Table 7.6: Accuracy precision approach.

\begin{tabular}{|c|c|c|c|c|c|c|}
\hline \multirow[b]{2}{*}{ Positioning } & \multicolumn{3}{|c|}{ Vertical Positioning Error } & \multicolumn{3}{|c|}{ Horizontal Positioning Error } \\
\hline & $\begin{array}{c}\text { Mean } \\
{[\mathrm{m}]}\end{array}$ & $\begin{array}{c}\text { Standard } \\
\text { deviation } \\
{[\mathrm{m}]}\end{array}$ & $\begin{array}{l}\text { Accuracy } \\
95 \%[\mathrm{~m}]\end{array}$ & $\begin{array}{c}\text { Mean } \\
{[\mathrm{m}]}\end{array}$ & $\begin{array}{c}\text { Standard } \\
\text { de viation } \\
\text { [m] }\end{array}$ & $\begin{array}{l}\text { Accuracy } \\
95 \%[\mathrm{~m}]\end{array}$ \\
\hline $\begin{array}{l}\text { Uncorrected } \\
\text { (GPS) CAT-I }\end{array}$ & 11.05 & 1.51 & 12.99 & 3.67 & 0.89 & 5.85 \\
\hline $\begin{array}{c}\text { Corrected } \\
\text { (GPS+GBAS) } \\
\text { CAT-I }\end{array}$ & 1.20 & 1.49 & 4.03 & 0.87 & 0.97 & 2.88 \\
\hline $\begin{array}{l}\text { Uncorrected } \\
\text { (GPS) APV }\end{array}$ & 10.70 & 1.96 & 13.37 & 3.05 & 0.37 & 3.05 \\
\hline $\begin{array}{c}\text { Corrected } \\
\text { (GPS+GBAS) } \\
\text { APV }\end{array}$ & 1.21 & 0.85 & 2.67 & 0.61 & 0.36 & 1.30 \\
\hline
\end{tabular}

The mean and standard deviation for each category are reduced when the differential corrections are applied, as indicated. Additionally, the accuracy requirement for GBAS is expressed as a 95th-percentile bound on navigation system error (NSE). The estimated values for this scenario and time interval for the vertical component were 4.03 and $2.67 \mathrm{~m}$ for CAT-I and APV, respectively, while the minimum requirement for GBAS CAT-I is $4 \mathrm{~m}$ and for APV is $8 \mathrm{~m}$ (Pullen, 2017). For horizontal component were $2.88 \mathrm{~m}$ and $1.30 \mathrm{~m}$ for CAT-I and APV, respectively, while the minimum requirement for GBAS CAT-I and APV is $16 \mathrm{~m}$. In this case, the vertical component exceeds the minimum requirement, influe nced 
by the spatial ionospheric gradient. This exemplifies how spatial gradients degrade positioning accuracy.

\section{Protection level results}

The sigma vertical ionospheric gradient $\left(\sigma_{\text {vert_iono_grad }}\right)$ is an ionospheric parameter evaluated for the computation of the protection levels (PL) at the aircraft. It is required to design and determine an upper bound for ionospheric spatial gradients which should be considered by the protection levels. It is relevant to mention that large spatial gradients often produce significant range errors through the carrier smoothing processing performed by a GBAS.

In this scenario, a sigma vertical ionospheric gradient $\sigma_{\text {vert_iono_grad }}=20$ $\mathrm{mm} / \mathrm{km}$ was applied, because this value was estimated from the Station-Pair method between the RIOD and ONRJ RBMC network stations on 20 December 2014. The $H P L(k)$ and $V P L(k)$ were estimated between 00:00:00 UTC and 00:10:00 UTC on 20 December 2014, based on the simulation system parameters described in Table 7.5. The protection levels were estimated using the mathematical formulation presented in Section 6.15 and the results are shown in Figures 7.26 and 7.27, for both components. In addition, the horizontal and vertical positioning error are also shown in each plot, respectively.

Figures 7.26 and 7.27 indicate that the horizontal and vertical protection level (green lines) decrease as the aircraft approaches the Rio de Janeiro Airport. This occurs due to the decrease of the ionospheric uncertainty, resulting from the simultaneous decreases of the distance to the ground station and the aircraft velocity (from $\mathrm{V}_{\text {air }}=100 \mathrm{~m} / \mathrm{s}$ to $\mathrm{V}_{\text {air }}=40 \mathrm{~m} / \mathrm{s}$, approximately), as shown in Figure 7.3.

For the horizontal component, HPL (green line) in Figure 7.27 always displays lower values than HAL for this scenario in both cases: APV and CAT-I (HAL $=40 \mathrm{~m}$ for CAT-I and APV). HPE (GPS with GBAS corrections) does not exceed the estimated HPL. In summary, this scenario can be represented by HPE(GPS+GBAS) $<$ HPL $<$ VPL. 


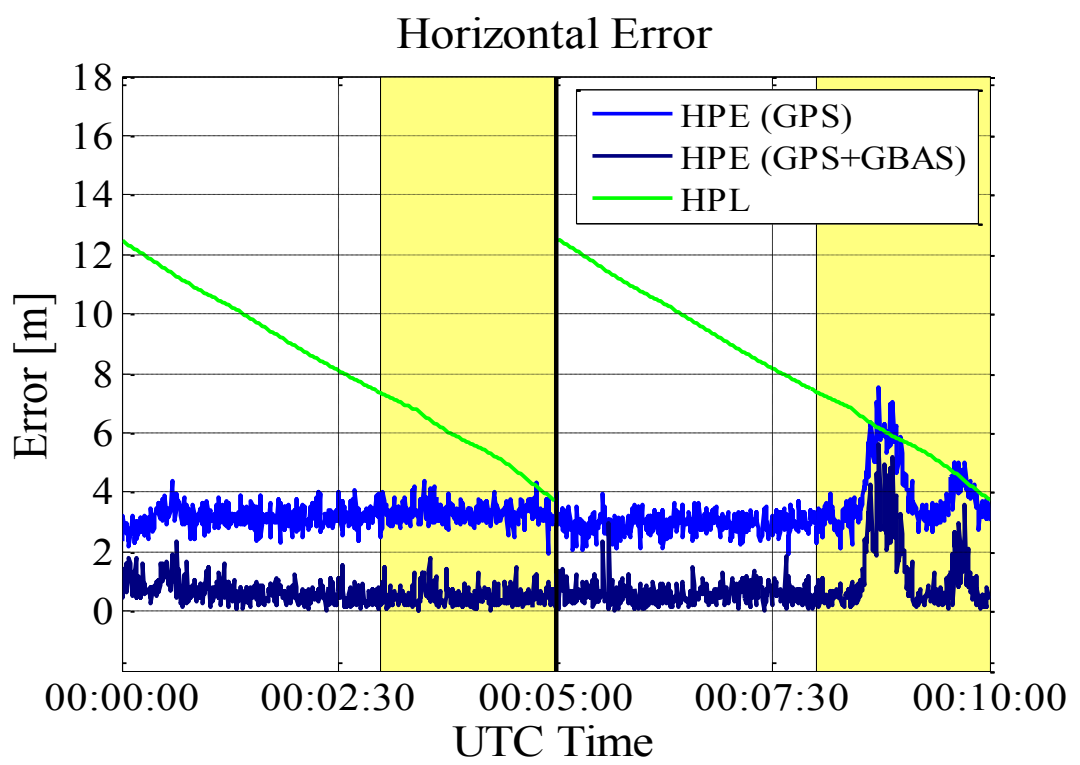

Figure 7.26: Horizontal positioning error and horizontal protection level of TAM/JJ3839 flight from 00:00:00 UTC to 00:10:00 UTC on 20 December 2014.

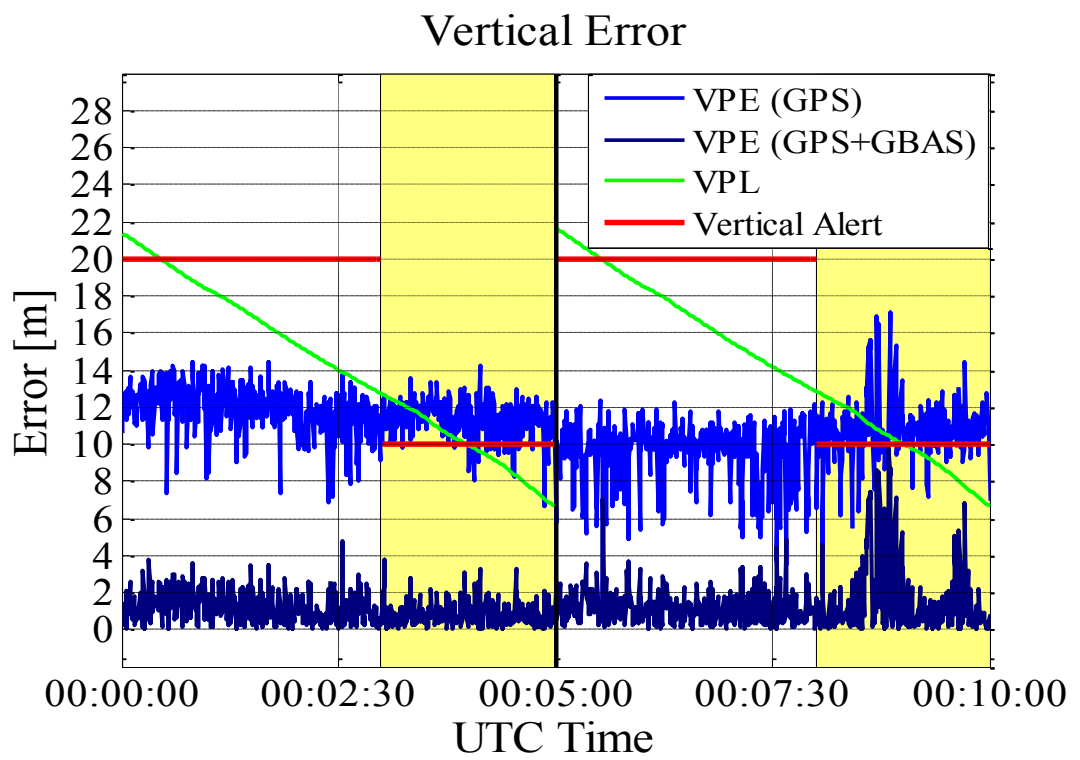

Figure 7.27: Vertical positioning error and vertical protection level of TAM/JJ3839 flight from 00:00:00 UTC to 00:10:00 UTC on 20 December 2014.

The vertical component VPL (green line), shown in Figure 7.27, exceeds the Vertical Alert Limit (VAL) of $10 \mathrm{~m}$ for CAT-I and $20 \mathrm{~m}$ for APV during large time intervals in both landings, reaching the maximum value of $21.6 \mathrm{~m}$ and $12.8 \mathrm{~m}$ for APV and CAT-I, respectively. It is observed that the VPE does not exceed the VPL during $10 \mathrm{~min}$. However, it is possible to identify during the second landing that the VPE values are near those of the VPL curve, induced by the spatial gradients. 
If the VPL is larger than the VAL, an integrity alert should be generated. The VPL is supposed to exceed the real unknown Vertical Position Error (VPE). If the VPE is larger than the VPL but smaller than the VAL, the information is misleading. If the VPE is larger than the VPL and also larger than the VAL, the information is hazardously misleading.

A correct estimation of $\sigma_{\text {vert_iono_grad }}$ using dense networks is very important, because the protection levels increase with this parameter. The impact of the scenario considered in this work was strong enough to limit the system availability. In this context, an inflated $\sigma_{\text {vert_iono_grad }}$ and its performance in a GBAS was carried out by Lee (2008). Additionally, an analysis and evaluation of $\sigma_{\text {vert_iono_grad }}$ in a static case using the Pegasus software was developed by Silva (2020).

\section{Integrity results}

This subsection estimates the integrity parameters. Integrity is defined by ICAO (1999) as a measure of the trust that can be placed in the correctness of the information supplied by a navigation system. The integrity parameters are characterized by Horizontal and Vertical Protection Levels, detailed in section 6.15.

An integrity plot is a visual form to represent the horizontal and vertical performance. This plot depicts the performance and integrity of the GBAS, indicating the number of times with adequate integrity to meet the categories of precision approximation. An integrity plot expresses the number of epochs of corresponding (PE, PL) values using color pixels, with $\mathrm{PE}$ in the $\mathrm{X}$-axis and $\mathrm{PL}$ in the Y-axis. The use of integrity plots is advantageous to verify the frequencies of misleading information (MI) and Hazardously Misleading Information (HMI) occurrence, which can be intuitively checked as is shown in Figure 7.28.

- A misleading information event occurs when, being the system declared available, the position error exceeds the protection level but not the alert limit.

- A hazardously misleading information event occurs when, being the system declared available, the position error exceeds the alert limit. 


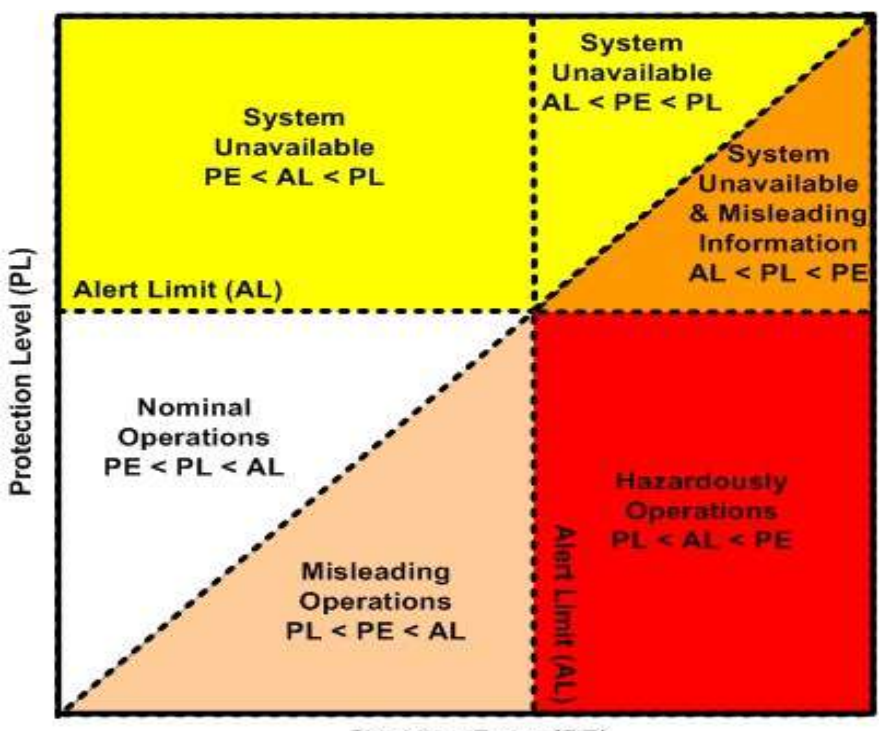

Position Error (PE)

Figure 7.28: Integrity plot

The results obtained and analyzed for the horizontal and vertical components in Figures 7.26 and 7.27 are presented in the integrity plots displayed in Figures 7.29 and 7.30. These plots show the performance of the GBAS at the Rio de Janeiro Airport for CAT-I during a period with high solar activity.

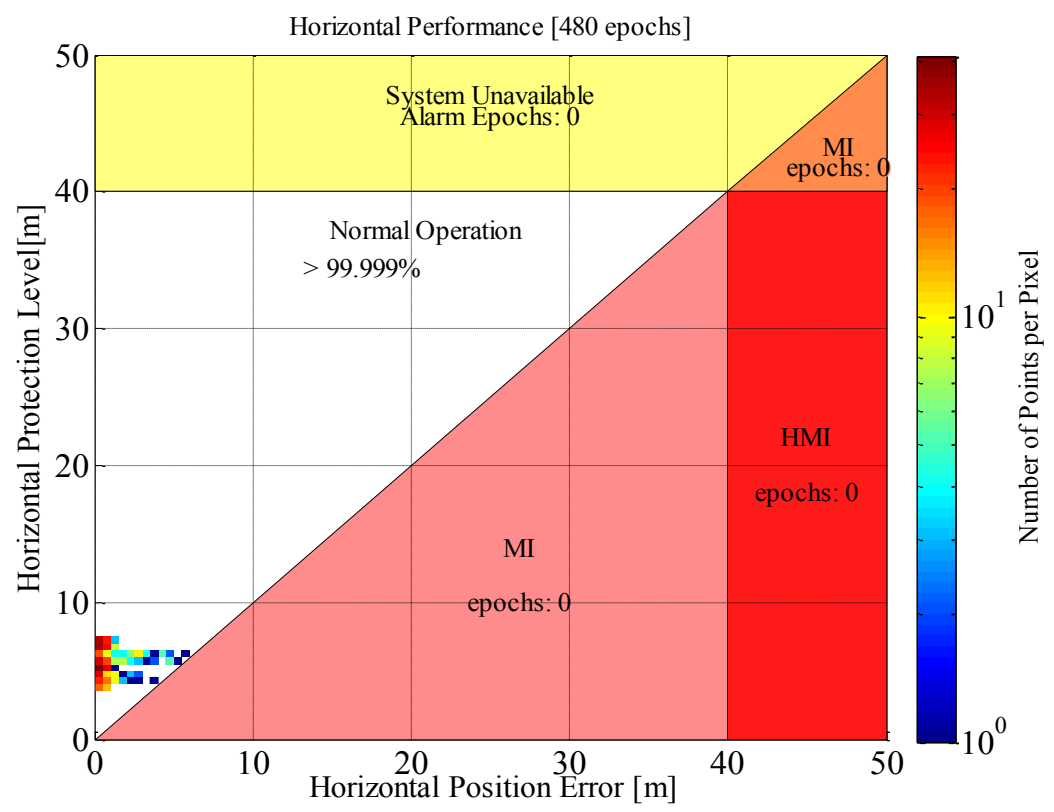

Figure 7.29: Integrity plot of the horizontal performance of TAM/JJ3839 flight for CAT-I from 00:00:00 UTC to 00:10:00 UTC on 20 December 2014. 


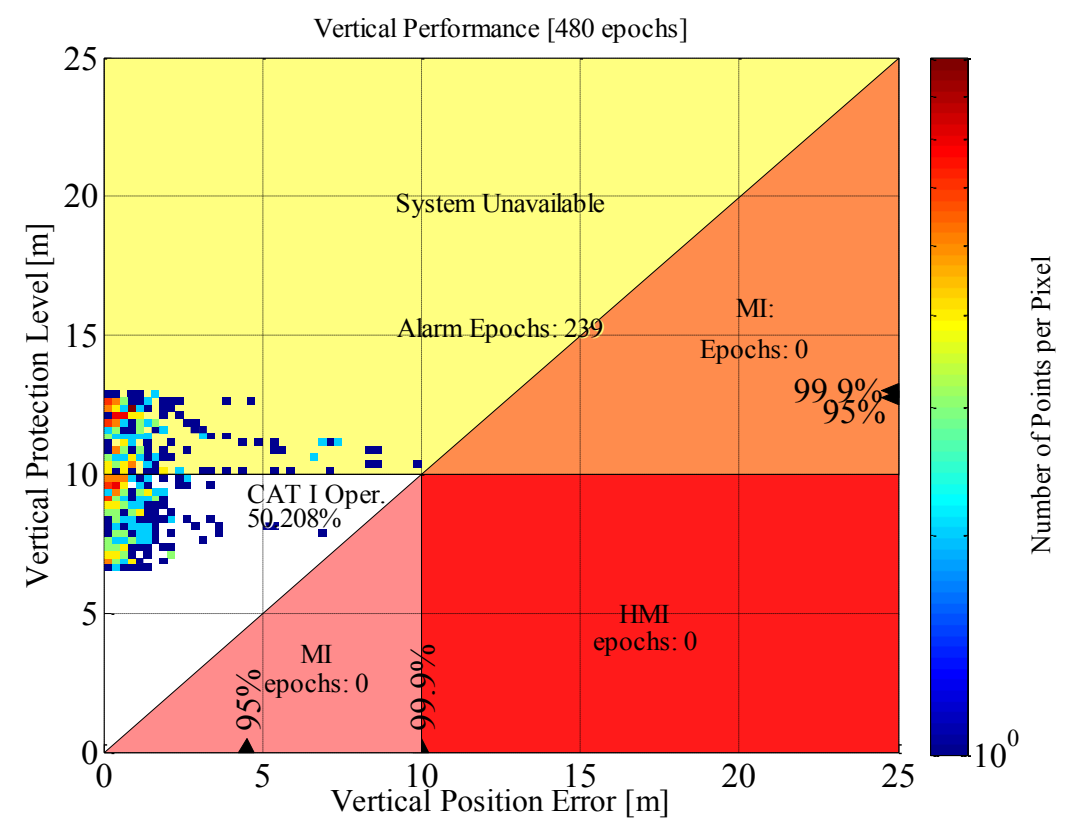

Figure 7.30: Integrity plot of the vertical performance of TAM/JJ3839 flight for

CAT-I from 00:00:00 UTC to 00:10:00 UTC on 20 December 2014.

For the horizontal component, Figure 7.29 shows that all the pairs (HPE, HPL) are located in the Normal Operation region, above the diagonal line HPL = HPE and below the horizontal line HPL $=40 \mathrm{~m}=\mathrm{HAL}$, even during the intervals of large spatial gradients. This plot reveals that the percentage of operation in CATI is higher than $99.999 \%$, due that all the 480 epochs corresponding to 2 landings and 2 min within the operation area for CAT-I (total of 4 minutes at 2 samples per second). Systems having this property are conservative.

For the vertical component, Figure 7.30 indicates that results from only 241 epochs $(50.208 \%)$ are within the Normal Operation area for CAT-I. This indicates a loss of the integrity condition for the approach category in the vertical component. Although the VPE did not exceed the VAL and VPL, the fact that for many VPL values are greater than the VAL reduces the GBAS availability for the precision approach category. Additionally, the integrity plot for the vertical component for CAT-I and APV is presented in Figure 7.31, considering that the corresponding requirements are more stringent than those for the horizontal component. 


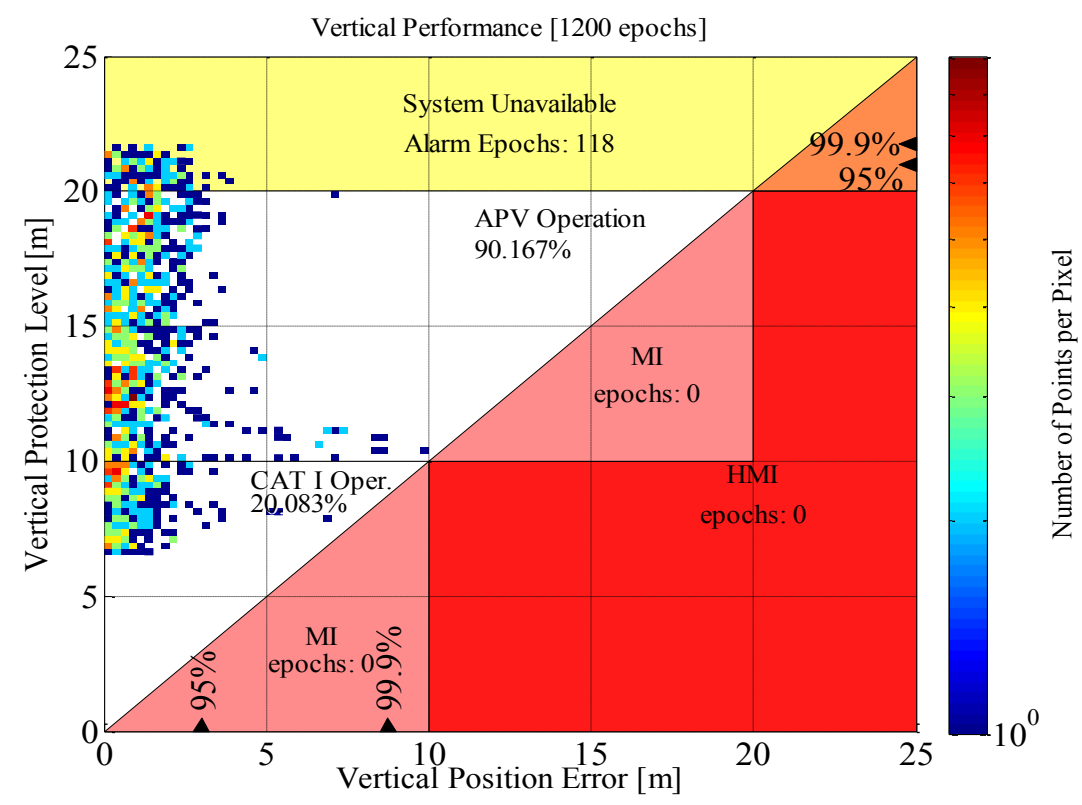

Figure 7.31: Integrity plot of vertical performance of TAM/JJ3839 flight for CATI and APV from 00:00:00 UTC to 00:10:00 UTC on 20 December 2014.

The values estimated for the VPL exceeded the VAL at 118 epochs, for a total of 1200 . The system has $90.167 \%$ of operability, indicating a loss of the integrity condition and becoming an unavailable system during $9.833 \%$ of the analyzed interval.

To summarize, the Rio de Janeiro Airport presents degradation of the accuracy and loss of the integrity condition during periods of high solar activity, reducing the availability of the GBAS. The parameter $\sigma_{\text {vert_iono_grad }}$ has a high influence in the results. Thus, a precise estimation of this parameter is important.

\subsubsection{Case study: Rio de Janeiro Airport, 22 July 2009}

In this section, results from the positioning performance are presented for the Rio de Janeiro Airport during the time interval from 00:00:00 UTC to 00:10:00 UTC on 22 July 2009 (a period of low solar activity during the winter solstice; thus, due to the combination of these two factors, strong scintillation is rarely expected). In the current study case, the aircraft tracked seven GPS satellites (SV 7, SV 13, SV 16, SV 20, SV 23, SV 25 and SV 32) during the described time interval. The skyplot of the GPS satellites is shown in the left panel of Figure 7.32. The right panel of the same Figure shows the ground projections of the GPS satellite positions, using the same color code and the corresponding IPPs, using red dots. 

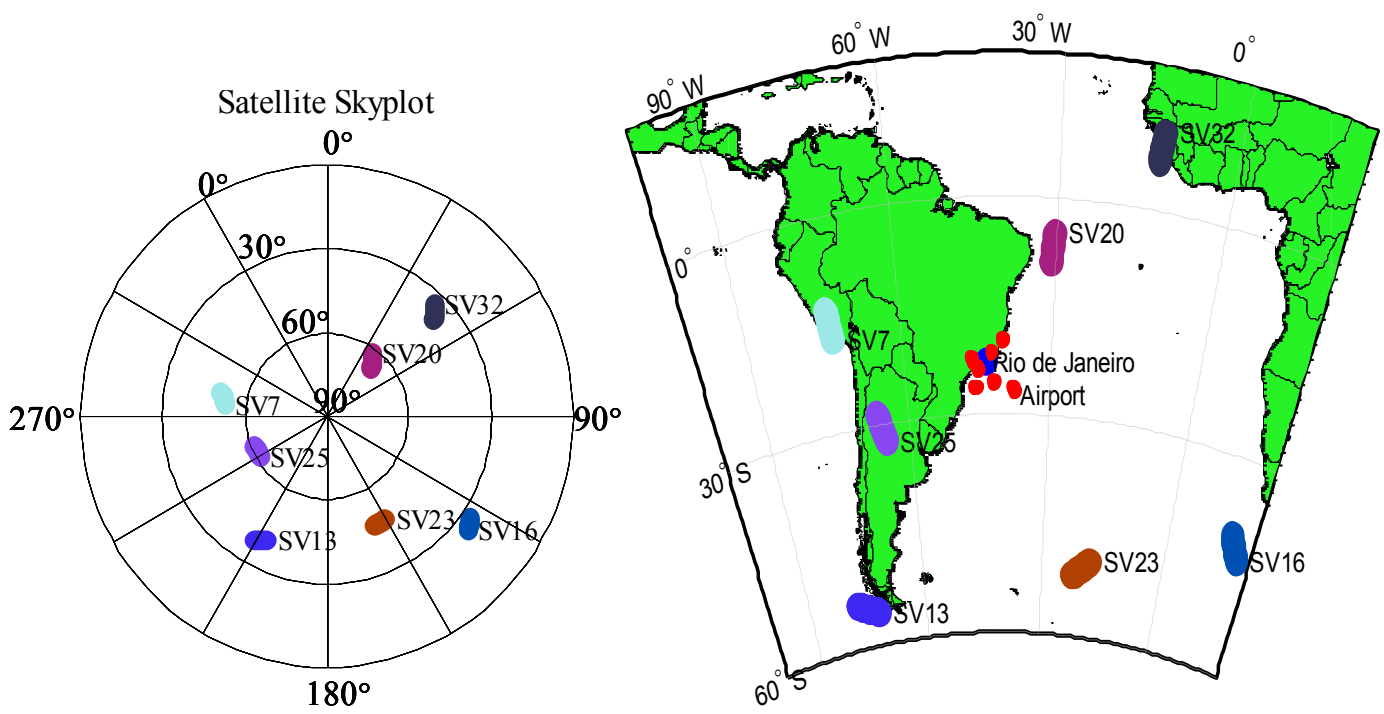

Figure 7.32: GPS satellite skyplot for TAM/JJ3839 flight from 00:00:00 UTC to 00:10:00 UTC on 22 July 2009 (Left); ground projections of the GPS satellite positions using the same color code and the corresponding IPPs using red dots (Right).

Following the same procedure of Section 7.1.3, time series of pseudorange $P R_{(i, j)}(k)$, carrier-phase $\phi_{(i, j)}(k)$ and received signal power $C_{(i, j)}(k)$ observables are generated for channels $(i, j)$ defined between GPS tracked satellites $(i=\mathrm{SV} 7$, SV 13, SV 16, SV 20, SV 23, SV 25 and SV 32) and the aircraft (j) between 00:00:00 UTC and 00:10:00 UTC on 22 July 2009, with time denoted by $(k)$. The effects associated with the observables were simulated based on the satellite positions, using the geophysical and environmental parameters described in Tables 7.7 and 7.8 , respectively.

Table 7.7: Geophysical parameters on 22 July 2009.

\begin{tabular}{|l|c|c|}
\hline \multirow{3}{*}{$\begin{array}{l}\text { Geophysical } \\
\text { parameters }\end{array}$} & Parameter & Value \\
\cline { 2 - 3 } & F10.7 index & 203 \\
\cline { 2 - 3 } & Kp index & $70[\mathrm{sfu}]$ \\
\hline
\end{tabular}


Table 7.8: Environmental parameters at the Rio de Janeiro Airport on 22 July 2009.

\begin{tabular}{|c|c|c|}
\hline & Parameter & Value \\
\hline \multirow{4}{*}{ Environment } & Air pressure & $1021[\mathrm{hPa}]$ \\
\cline { 2 - 3 } & Temperature & $22^{\circ} \mathrm{C}$ \\
\cline { 2 - 3 } & Humidity & $70 \%$ \\
\cline { 2 - 3 } & Refractivity $N$ & 370 \\
\cline { 2 - 3 } & Refractivity Uncertainy & 15 \\
\cline { 2 - 3 } & Tropospheric scale height & $12900 \mathrm{~m}$ \\
\hline
\end{tabular}

Additionally, ionospheric gradients $\left(\Delta I / d_{I P P}\right)$ are added to the ionospheric delay term to represent spatial variations of the ionosphere. Series of spatial gradients are extracted from RIOD and ONRJ RBMC stations using the StationPair method $\left(g^{s}\right)$ during the present time interval, as reported in Section 4.3. Note that these gradients are added to the ionospheric delay term $I_{(i, j)}(k)$ of the pseudorange and carrier-phase observable simulated for the aircraft corresponding to each channel, considering the distance between IPP aircraft and IPPRSMU1.

At every integer minute, the vector of amplitude scintillation indices $\mathrm{S}_{4}$ for the available satellites were sorted according to the distribution associated with the label AA-LO (scintillation season from April to August, for low solar activity) displayed by Figure 5.39. The corresponding time series are shown in Figure 7.33. The corresponding vectors of standard deviation of phase scintillation $\sigma_{\varphi}$, sorted from the corresponding values of $\mathrm{S}_{4}$ as prescribed at the end of Section 5.1.6, in association with Figure 5.10. The corresponding time series of $\sigma_{\varphi}$ vectors are represented in Figure 7.34. 


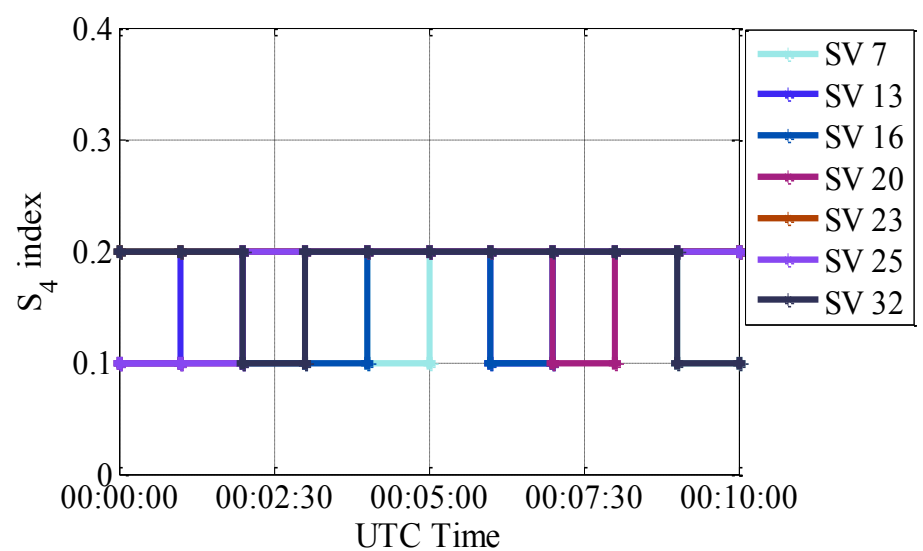

Figure 7.33: Time series of amplitude scintillation index $\mathrm{S}_{4}$ for each GPS satellite, with 1-min resolution.

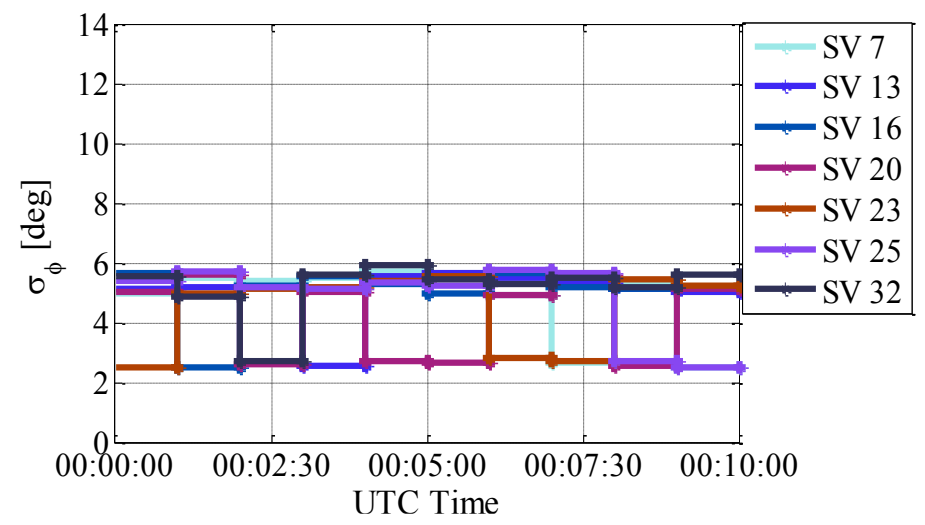

Figure 7.34: Time series of standard deviation of phase scintillation $\sigma_{\varphi}$ for each GPS satellite, with 1-min resolution.

Next, time series of amplitude scintillation and phase scintillation are generated using the index $\mathrm{S}_{4}$ and $\sigma_{\varphi}$; and added to the received signal model and carrier-phase model, respectively. Figure 7.35 and 7.36 show pseudorange $P R_{(i, j)}(k)$ and received signal power $C_{(i, j)}(k)$ observables. 


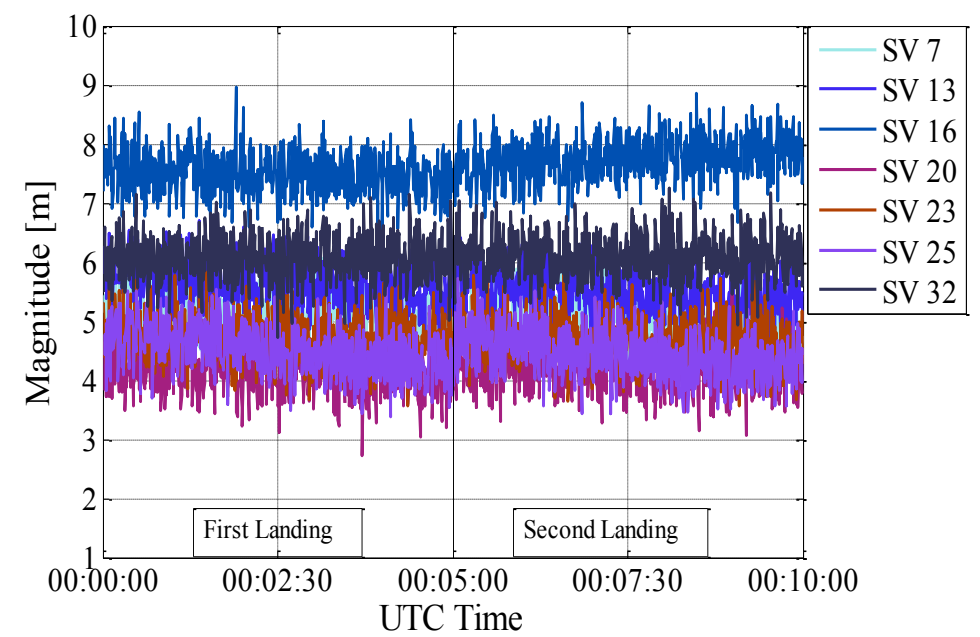

Figure 7.35: Time series of the effects due to ionospheric and tropospheric delays, multipath and random errors on the pseudorange, generated for TAM/JJ3839 flight from 00:00:00 UTC to 00:10:00 UTC on 22 July 2009.

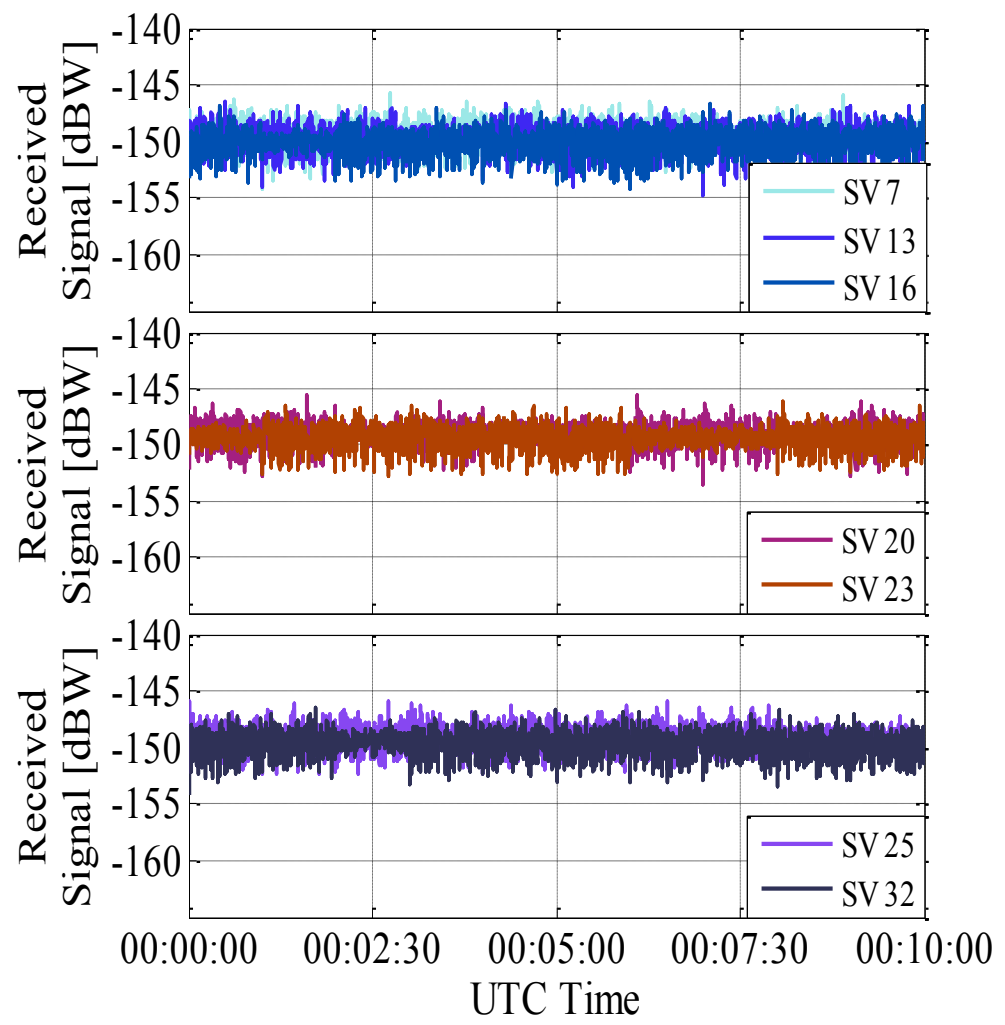

Figure 7.36: Time series of the received signal power, generated for TAM/JJ3839 flight from 00:00:00 UTC to 00:10:00 UTC on 22 July 2009, considering the effects due to amplitude scintillation, multipath, and random errors.

The magnitudes of the effects associated with the signal delays of GPS satellites during the period of low solar activity do not exceed $9 \mathrm{~m}$, as shown by 
Figure 7.35. The maximum delay is found for SV 16, observed from the aircraft position with low elevation angles (approximately $28^{\circ}$ ), as displayed by the skyplot of Figure 7.32. Analyzing Figures 7.7 and 7.35, the differences between the magnitudes of the delay due to the associated effects are evident. The maximum delay observed during the high solar activity interval is $15.84 \mathrm{~m}$, which is 1.75 times greater than the one associated with the present low solar activity conditions. All the ionospheric delay estimated at each IPP corresponding to the GPS satellite are related to the TEC as is depicted in Figure 7.37.

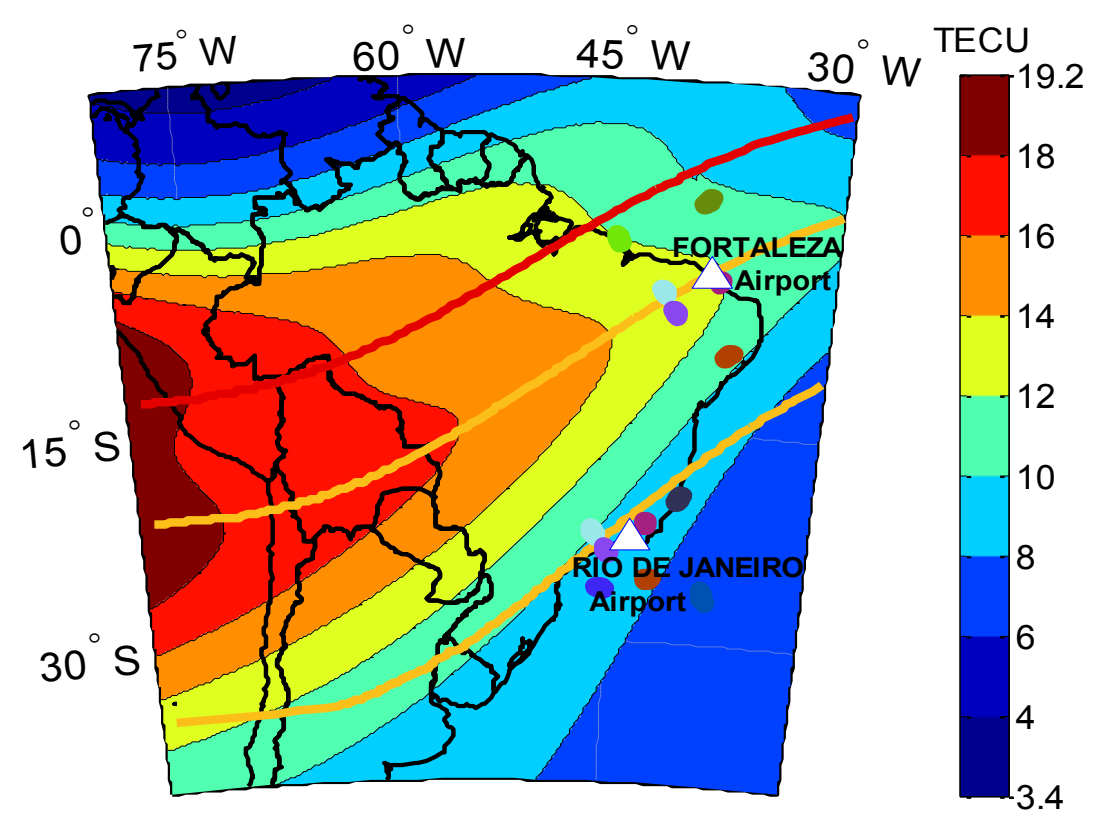

Figure 7.37: IRI+Residual TEC Map and ground projections of the corresponding IPPs at 00:10:00 UTC on 22 July 2009.

Each subplot in Figure 7.36 displays 10-min time series of received signal power generated for seven tracked GPS satellites. The amplitude scintillation was simulated with basis on the $\alpha-\mu$ statistical distribution associated to the $\mathrm{S}_{4}$ index estimated by Moraes et al. (2017). All the simulated signals kept the same range of $\mathrm{S}_{4}$ indices $\left(\mathrm{S}_{4} \leq 0.3\right)$. The three panels of Figure 7.36 show that the magnitude of deep fades are very similar for the seven GPS satellites. The 10-min times series of received signal power simulated in Figure 7.36 present shallower scintillation fadings in comparison with the ones generated during the period with high solar activity, displayed in Figure 7.9. 
Similarly, time series of pseudorange, carrier phase and received signal power were generated for the four GBAS receivers located at the Rio de Janeiro Airport (depicted in Figure 7.1) during the present time interval. In sequence, The GBAS monitor performed the same tests and generated differential corrections, as discussed in more detail by Section 7.1.3. The results from the pseudorange corrections $P R_{\operatorname{corr}(n)}(k)$ estimated for each validated GPS satellite in the current study case are shown in Figure 7.38.

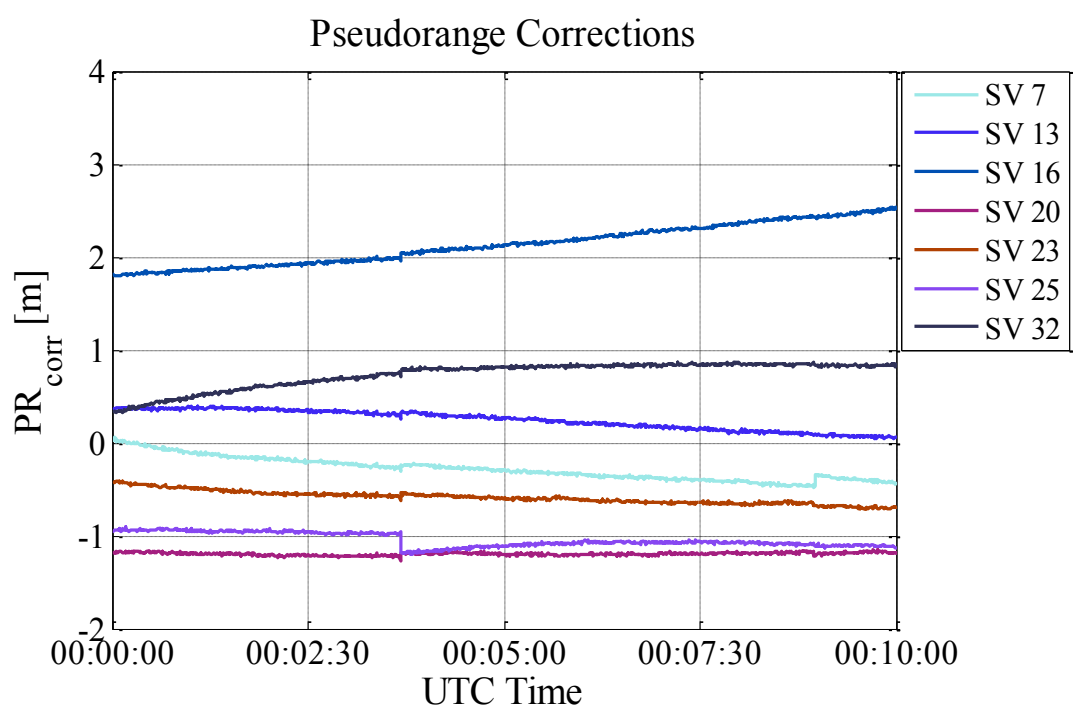

Figure 7.38: Pseudorange corrections estimated by the GBAS during TAM/JJ3839 flight from 00:00:00 UTC to 00:10:00 UTC on 22 July 2009.

The pseudorange corrections $P R_{\operatorname{corr}(n)}(k)$ generated during the low solar activity interval present lower magnitudes than those estimated during high solar activity. Indeed, SV 16 presents a differential correction of 2.5 meters at 00:10:00 UTC. It is observed that, since the ionosphere is now less perturbed, the present differential corrections do not display the fluctuations observed during the high solar activity interval.

Next, the aircraft positions are estimated based on uncorrected and GBAScorrected pseudoranges. The positioning errors are estimated in NED coordinates, using the aircraft path positions as references. The results are shown in Figure 7.39. 


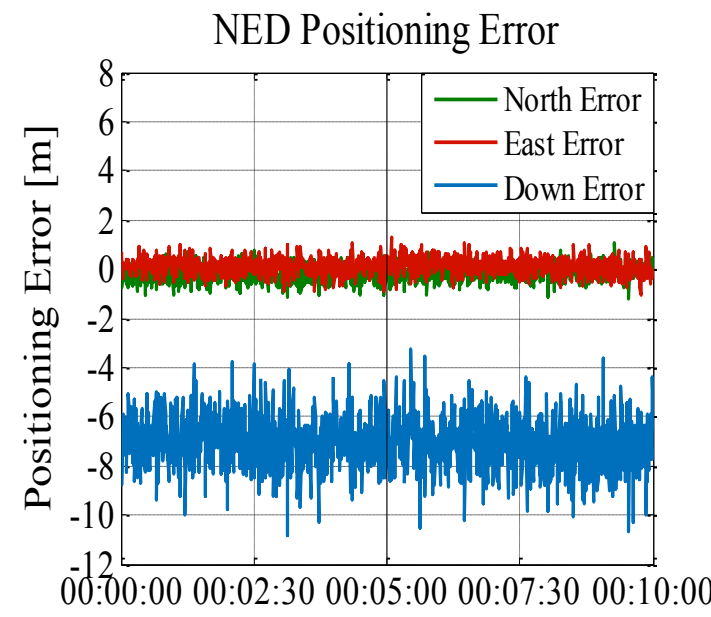

UTC Time

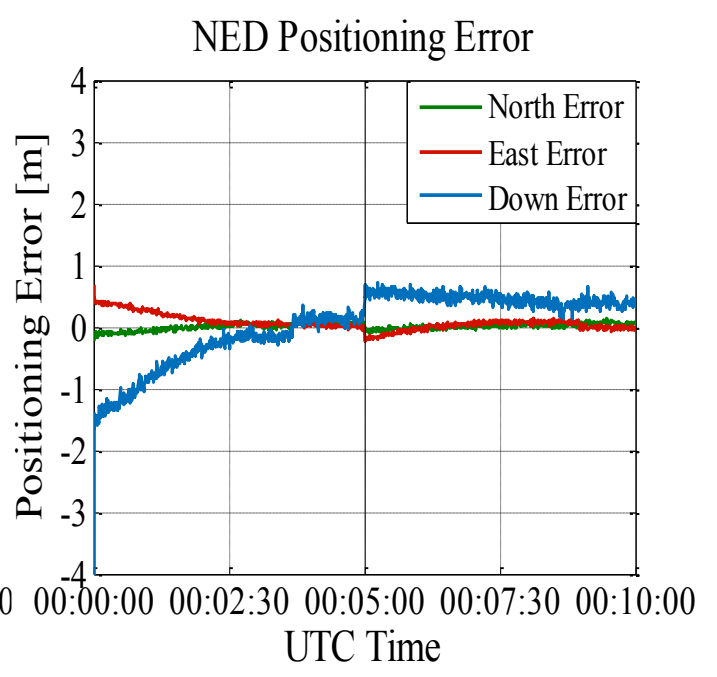

Figure 7.39: NED positioning errors based on uncorrected (left) and GBAScorrected pseudoranges (right) during TAM/JJ3839 flight from 00:00:00 UTC to 00:10:00 UTC on 22 July 2009.

Horizontal and vertical positioning error are then estimated based on the generated observables and corrected pseudoranges, as shown in Figures 7.40 and 7.41 , respectively. In the present scenario, a sigma vertical ionospheric gradient $\sigma_{\text {vert_iono_grad }}=4 \mathrm{~mm} / \mathrm{km}$ was adopted, according to the values estimated from the Station-Pair method between the RIOD and ONRJ RBMC network stations on 22 July 2009. The horizontal and vertical protection level were estimated from 00:00:00 UTC to 00:10:00 UTC on 22 July 2009 based on the simulation system parameters described in Table 7.8. The results are represented by the green curves in Figures 7.40 and 7.41.

For the horizontal component, Figure 7.40 shows that the HPE estimated using uncorrected pseudoranges (blue line) and corrected observables (dark blue line) present lower values than the HPL (green line) during the intervals of time for both landings. Similarly, the estimated values for HPL and HPE do not exceed the horizontal alert limit (HAL), that represents the maximum tolerable error, equal to $40 \mathrm{~m}$ for CAT-I and APV. To display HPE and HPL with an expanded vertical scale, HAL is not represented in Figure 7.40. 


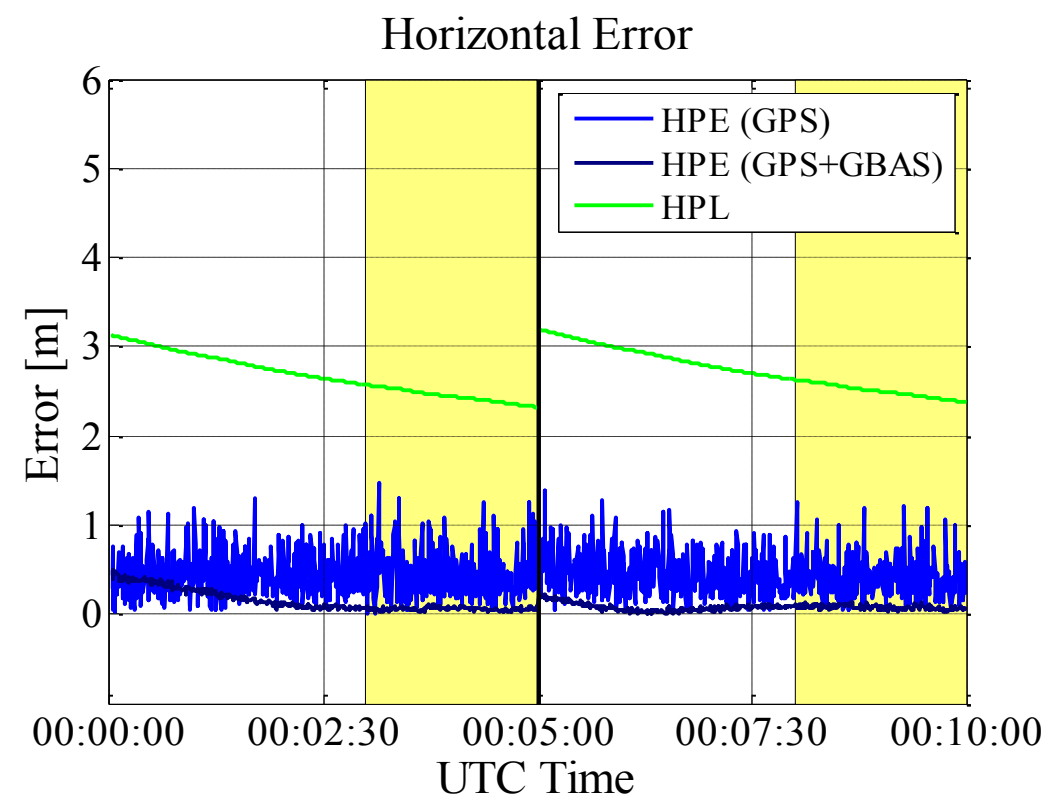

Figure 7.40: Horizontal positioning error and horizontal protection level for TAM/JJ3839 flight from 00:00:00 UTC to 00:10:00 UTC on 22 July 2009.

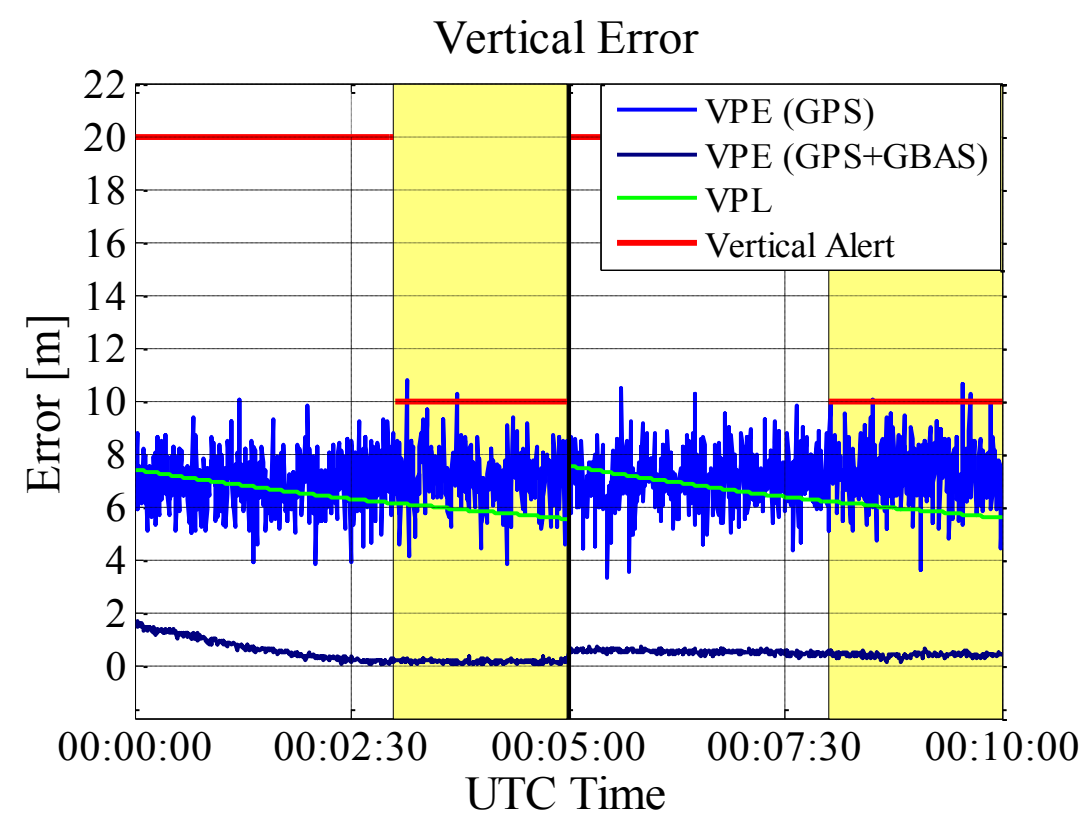

Figure 7.41: Vertical positioning error and vertical protection level for TAM/JJ3839 flight from 00:00:00 UTC to 00:10:00 UTC on 22 July 2009.

For the vertical component, Figure 7.41 shows that the VPE estimated for uncorrected pseudorange (blue line) exceeds the VPL (green line) during most of the time. It is for this reason that corrections are necessary. On the other hand, values estimated for VPE for GBAS-corrected pseudoranges (dark blue) do not 
exceed the VPL values. In addition, VPL and VPE do not exceed the constant value of $10 \mathrm{~m}$ (red line) of the vertical alert limit (VAL). Therefore, the following inequality holds: VPE $<$ VPL $<$ VAL.

After the process of correction, the horizontal and vertical position errors based on corrected pseudoranges are small, it is due that the corrections reduced the horizontal and vertical errors between approximately $75 \%$ and $99 \%$ as is shown in Figures 7.40 and 7.41 respectively.

The system performances in the horizontal and vertical component at the Rio de Janeiro Airport are shown in the integrity plots of Figures 7.42 and 7.43, respectively.

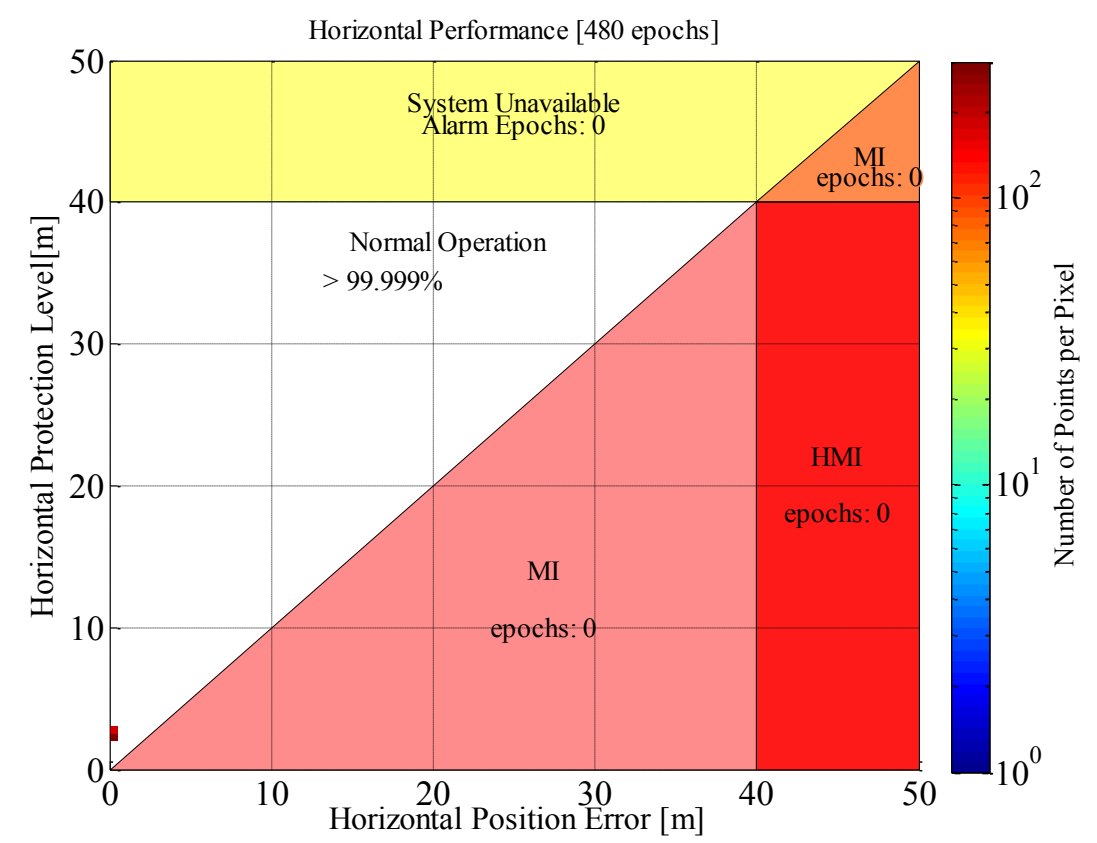

Figure 7.42: Integrity plot of the horizontal performance of TAM/JJ3839 flight for CAT-I from 00:00:00 UTC to 00:10:00 UTC on 22 July 2009. 


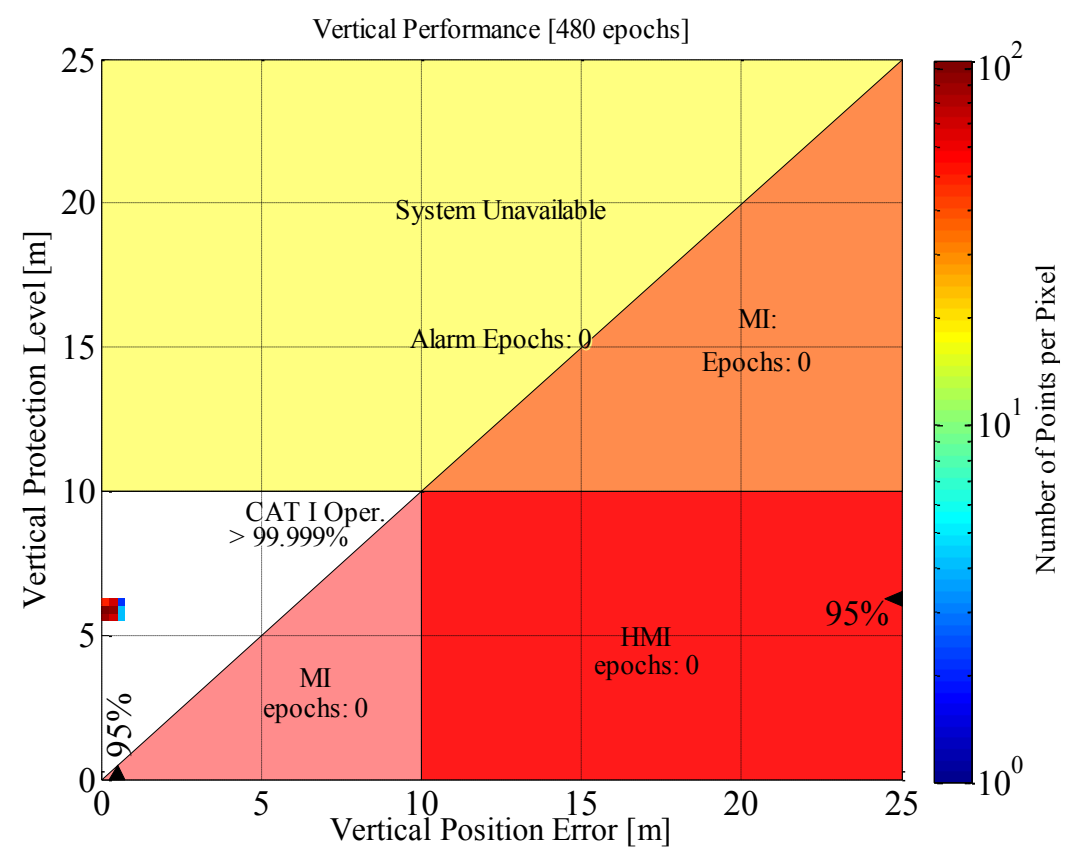

Figure 7.43: Integrity plot of vertical performance of TAM/JJ3839 flight for CATI from 00:00:00 UTC to 00:10:00 UTC on 22 July 2009.

Figure 7.42 indicates that the obtained values are within the operational area for CAT-I approach, according to the thresholds characterized by HPE $<$ HPL $<$ HAL. This condition is present during $99.999 \%$ of the epochs analyzed for CAT-I. The operational condition of $100 \%$ estimated for horizontal component agrees with the results presented by Silva (2020) for a scenario with similar characteristics using a $\sigma_{\text {vert_iono_grad }}$ from $8 \mathrm{~mm} / \mathrm{km}$ to $20 \mathrm{~mm} / \mathrm{km}$.

Similarly, Figure 7.43 shows that $100 \%$ of the epochs are within the operational area for APV and CAT-I, indicating an operational condition of integrity for the approach category in the vertical component due (VPE $<$ VPL $<$ VAL). The results presented in Figures 7.42 and 7.43 are very concentrated. Thus, both components present low standard deviation.

The current analys is suggests that the GBAS deployed at the Rio de Janeiro Airport would not present accuracy and integrity degradations for both landings and APV and CAT-I approaches during low solar activity intervals. 


\subsection{Aircraft Positioning at the Pinto Martins International Airport, Fortaleza, Brazil}

Next, a second scenario is studied. The positions of approach and landing of one aircraft to the Pinto Martins International Airport (SBFZ) located in Fortaleza, Brazil, are estimated during a commercial flight to evaluate the performance of the GBAS simulation model under different geophysical conditions. This airport was selected because it is located in a region close to the geomagnetic equator, where EPBs are generated.

Similar to the case of the Rio de Janeiro Airport, results from another study case is discussed: 00:00:00 UTC to 00:10:00 UTC on 20 December 2014 (high solar activity) to analyze ionospheric effects. More detailed descriptions of the environment will be provided in the corresponding Sections.

\subsubsection{Scenario}

The Fortaleza Airport (SBFZ) does not actually operate an installed GBAS. For this scenario, the fixed positions of the four receivers and the main GBAS monitor are simulated. The receivers are denoted by RSMU1, RSMU2 RSMU3 and RSMU4, being distributed around the runway of the Fortaleza Airport as shown in Figure 7.44. In addition, the geodetic coordinates and DIP latitude of each receiver antenna are indicated in Table 7.9.

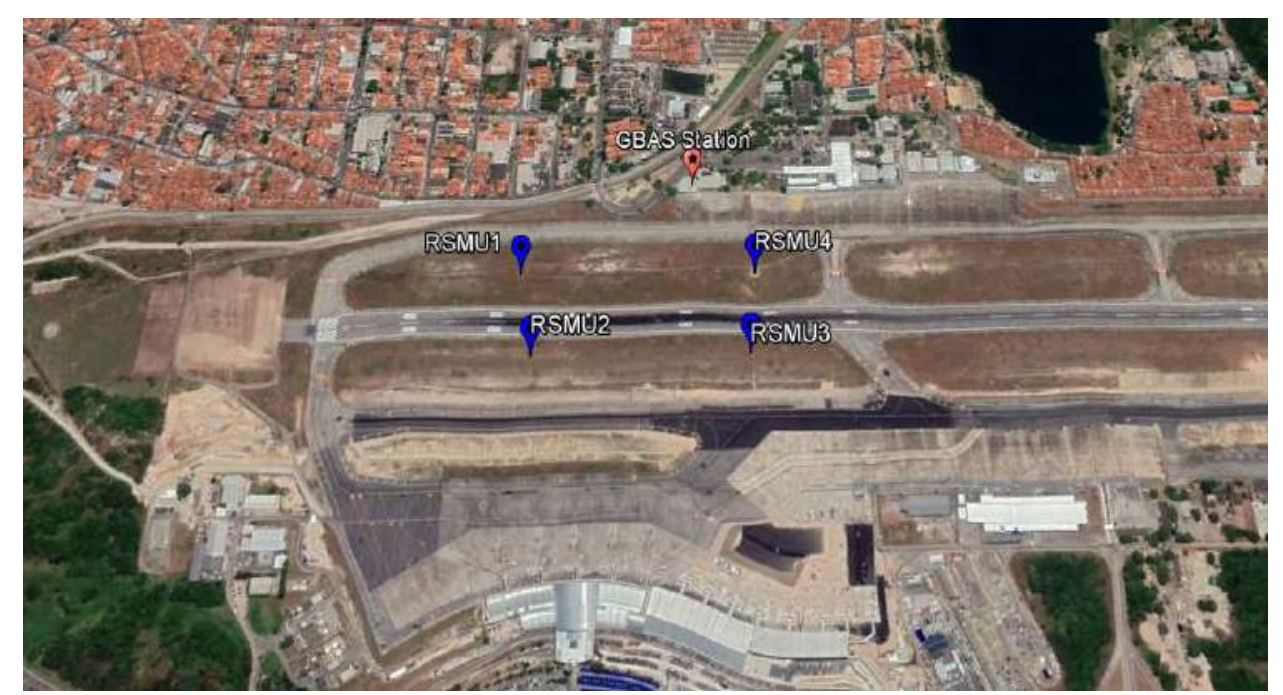

Figure 7.44: Simulated positions of GBAS monitor and reference antennas at the Pinto Martins International Airport, Fortaleza, Brazil [Adapted from Google Earth]. 
Table 7.9: Positions of reference antennas at the Fortaleza Airport.

\begin{tabular}{|c|c|c|c|}
\hline Receiver Station & Latitude & Longitude & Dip latitude \\
\hline RSMU1 & $3.7733^{\circ} \mathrm{S}$ & $38.5404^{\circ} \mathrm{W}$ & $7.5643^{\circ} \mathrm{S}$ \\
\hline RSMU2 & $3.7747^{\circ} \mathrm{S}$ & $38.5405^{\circ} \mathrm{W}$ & $7.5656^{\circ} \mathrm{S}$ \\
\hline RSMU3 & $3.7756^{\circ} \mathrm{S}$ & $38.5372^{\circ} \mathrm{W}$ & $7.5683^{\circ} \mathrm{S}$ \\
\hline RSMU4 & $3.7743^{\circ} \mathrm{S}$ & $38.5367^{\circ} \mathrm{W}$ & $7.5674^{\circ} \mathrm{S}$ \\
\hline
\end{tabular}

\subsubsection{Flight Data}

This simulation uses the approach and landing information of one aircraft to the Fortaleza Airport. The latitude, longitude, altitude and speed data of a commercial flight (AZU5346) operated by AZUL airlines were used and transformed into ECEF coordinates. This flight actually occurred on 04 June 2020, departing from the Recife International Airport (SBRF). Table 7.10 presents more information on the selected flight and Figure 7.45 shows the last section of the flight path. The data of this flight can be accessed on the FlightAware website (https $/ /$ pt. flightaware.com).

Figure 7.45 identifies the last tridimensional section of the flight path for the flight AZU5346 before the aircraft landing at the Fortaleza Airport (approximately 24 kilometers) by the yellow line limited between the blue and red markers. The last section of the flight path presents a time interval between 13:23:05 UTC and 13:28:05 UTC.

Table 7.10: Information on the AZU5346 flight.

\begin{tabular}{|c|c|}
\hline Flight & AZU5346 \\
\hline Date & 04 June 2020 \\
\hline Aircraft & Airbus 32Q \\
\hline Departure Airport & Recife International Airport (SBRF) \\
\hline Departure Time & $12: 32: 29$ UTC \\
\hline Arrival Airport & Fortaleza International Airport (SBFZ) \\
\hline Arrival Time & $13: 28: 05$ UTC \\
\hline
\end{tabular}




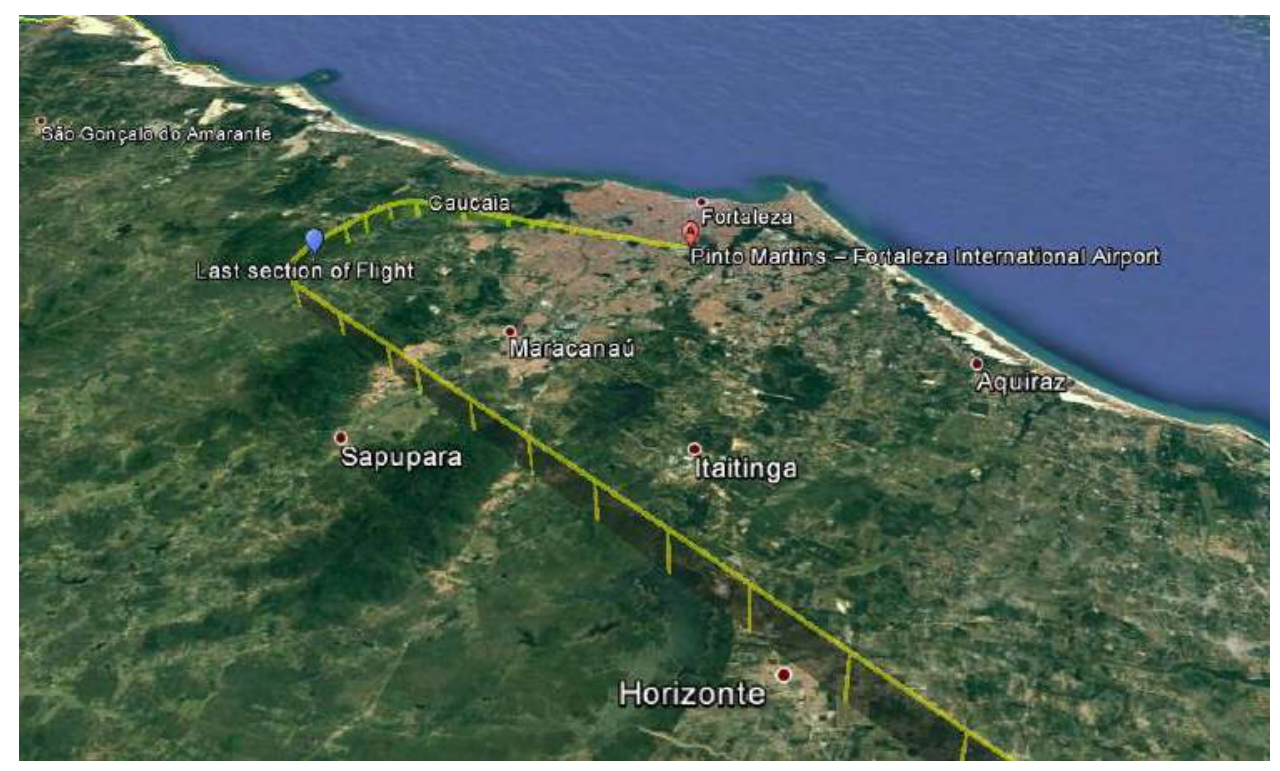

Figure 7.45: Path of the last section of Flight AZU5346.

[Adapted from Google Earth]

Thus, the positioning results will be based on pseudoranges, the combination of pseudoranges and GBAS corrections, as well as on true aircraft position information during the last 5 minutes of the flight path that corresponds to the precision approach categories: APV I, CAT-I, CAT-II and CAT-III, according to ICAO. The present work will simulate two 5-minute aircraft landings, representing a total interval of 10 minutes, emphasizing CAT-I requirements.

\subsubsection{Case study: Fortaleza Airport, 20 December 2014}

The objective of this Section is to evaluate the variation of the pseudorange corrections and positioning errors at an airport located close to the geomagnetic equator during a period of high solar activity. Positioning results are presented for the Fortaleza Airport during the time interval between 00:00:00 UTC and 00:10:00 UTC on 20 December 2014. In the current study case, the GBAS and aircraft receivers tracked seven GPS satellites during the described flight time interval (SV 14, SV 15, SV 18, SV 21, SV 22, SV 25 and SV 29). The skyplot of GPS satellites is shown in the left panel of Figure 7.46. The right panel of the same Figure shows the ground projections of the corresponding IPPs using dots of the same color code. 

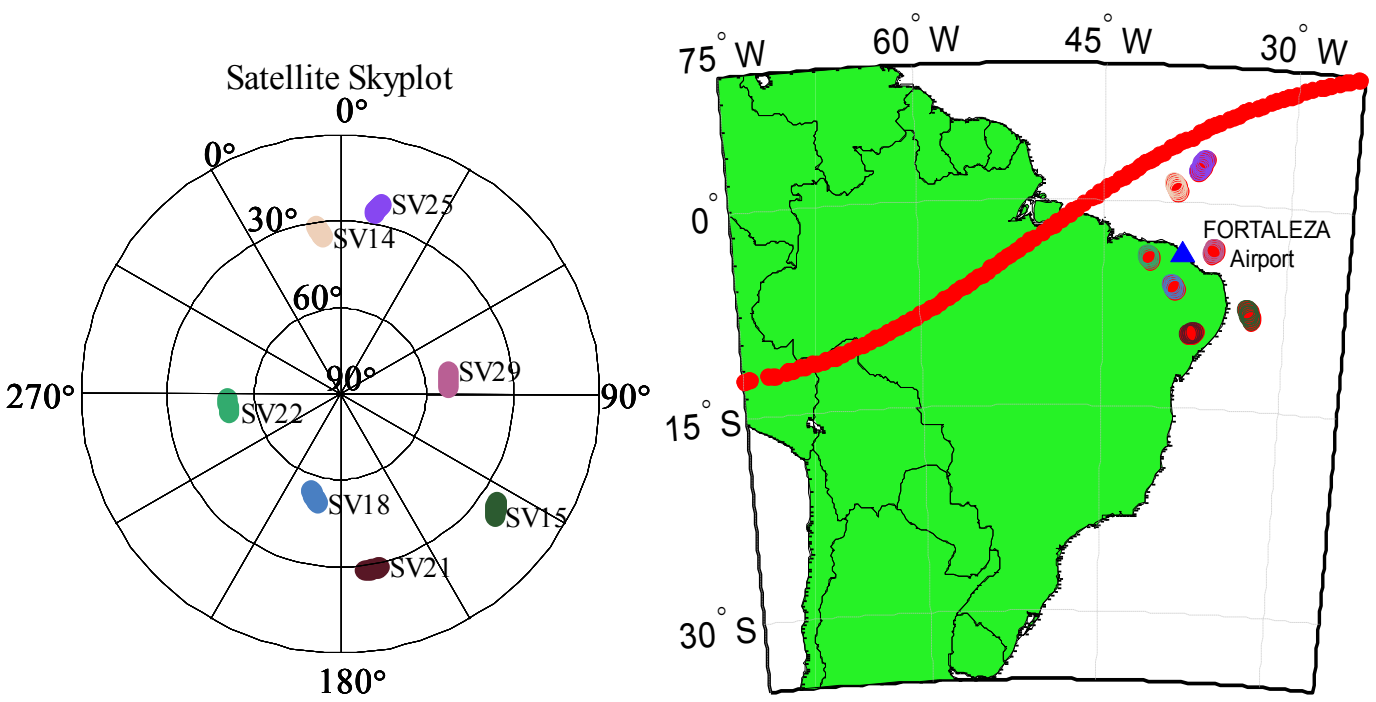

Figure 7.46: GPS satellite skyplot for AZU5346 flight from 00:00:00 UTC to 00:10:00 UTC on 20 December 2014 (Left); ground projections of the corresponding IPPs using dots of the same color code (Right).

Following the same procedure of Section 7.1.3, time series of pseudorange $P R_{(i, j)}(k)$, carrier-phase $\phi_{(i, j)}(k)$ and received signal power $C_{(i, j)}(k)$ observables are generated for channels $(i, j)$ defined between GPS tracked satellites ( $i=\mathrm{SV} 14$, SV 15, SV 18, SV 21, SV 22, SV 25 and SV 29) and the aircraft (j) between 00:00:00 UTC and 00:10:00 UTC on 20 December 2014, with time denoted by $(k)$. The effects associated with the observables were simulated based on the satellite positions, using the geophysical, environment and system parameters listed in Tables 7.3, 7.11 and 7.5, respectively.

Table 7.11: Environment parameters at the Fortaleza Airport on 20 December 2014.

\begin{tabular}{|c|c|c|}
\hline & Parameter & Value \\
\hline \multirow{4}{*}{ Environment } & Air pressure & $1013[\mathrm{hPa}]$ \\
\cline { 2 - 3 } & Temperature & $28^{\circ} \mathrm{C}$ \\
\cline { 2 - 3 } & Humidity & $70 \%$ \\
\cline { 2 - 3 } & Refractivity $N$ & 370 \\
\cline { 2 - 3 } & Refractivity Uncertainty & 15 \\
\cline { 2 - 3 } & Tropospheric scale height & $12900 \mathrm{~m}$ \\
\hline
\end{tabular}


In addition, ionospheric gradients $\left(\Delta I / d_{I P P}\right)$ are added to the ionospheric delay to represent spatial ionospheric variations. Series of spatial gradients were extracted from CEEU and CEFT stations (located in Fortaleza) using the StationPair method $\left(g^{s}\right)$ corresponding to the time interval from 00:00:00 UTC to 00:10:00 UTC on 20 December 2014, as reported in Section 4.3. These gradients are added to the ionospheric delay term $I_{(i, j)}(k)$ of the pseudorange and carrierphase observables simulated for the aircraft corresponding to each channel, considering the distance between IPP aircraft $_{\text {and IPP }}$ RSMU1.

At every integer minute, the vector of amplitude scintillation indices $\mathrm{S}_{4}$ for the available satellites were sorted according to the distribution associated with the label SM-HG (scintillation season from September to March, for high solar activity), similar to those displayed in Figure 5.39. However, the distribution from PALM station was used, because it is located in the same dip-latitude range. The corresponding time series are displayed in Figure 7.47. Also, the corresponding vectors of standard deviation of phase scintillation $\sigma_{\varphi}$, sorted from the corresponding values of $\mathrm{S}_{4}$ as already described, are shown in Figure 7.48.

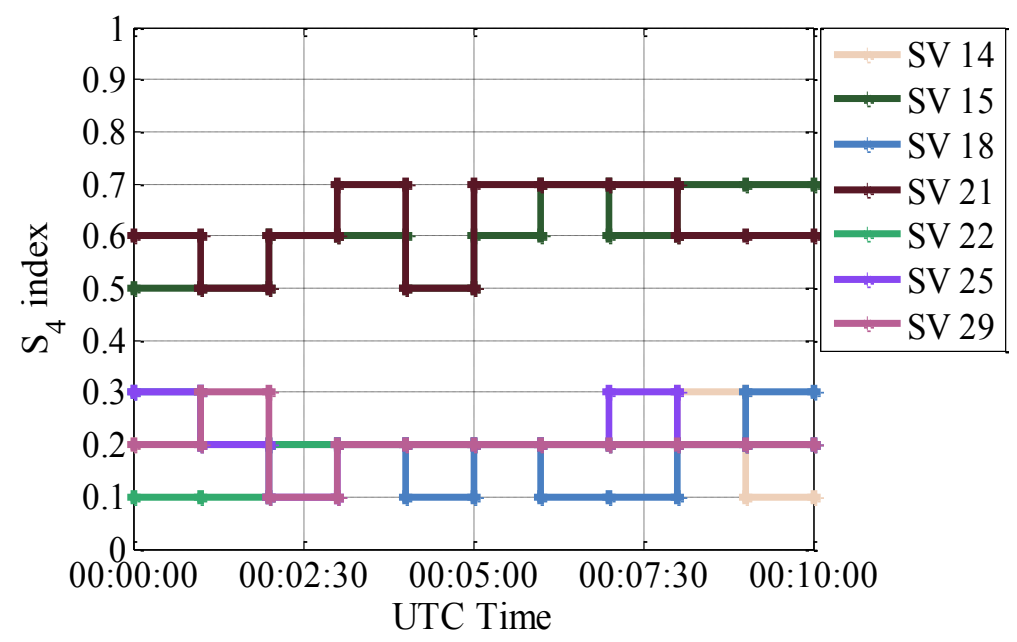

Figure 7.47: Time series of amplitude scintillation index $\mathrm{S}_{4}$ for each GPS satellite, with 1-min resolution. 


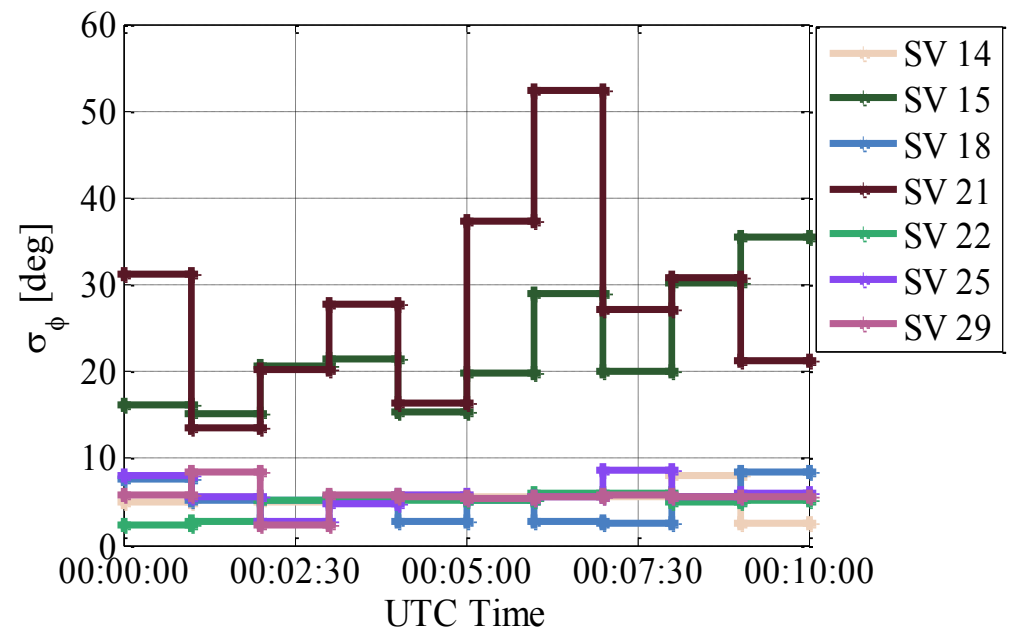

Figure 7.48: Time series of standard deviation of phase scintillation $\sigma_{\varphi}$ for each GPS satellite, with 1-min resolution.

Next, time series of amplitude and phase scintillation are generated and added to the received signal power model and to the carrier phase observables, respectively. Figure 7.49 and 7.50 show pseudorange $P R_{(i, j)}(k)$ and received signal power $C_{(i, j)}(k)$ observables for the first and second landing.

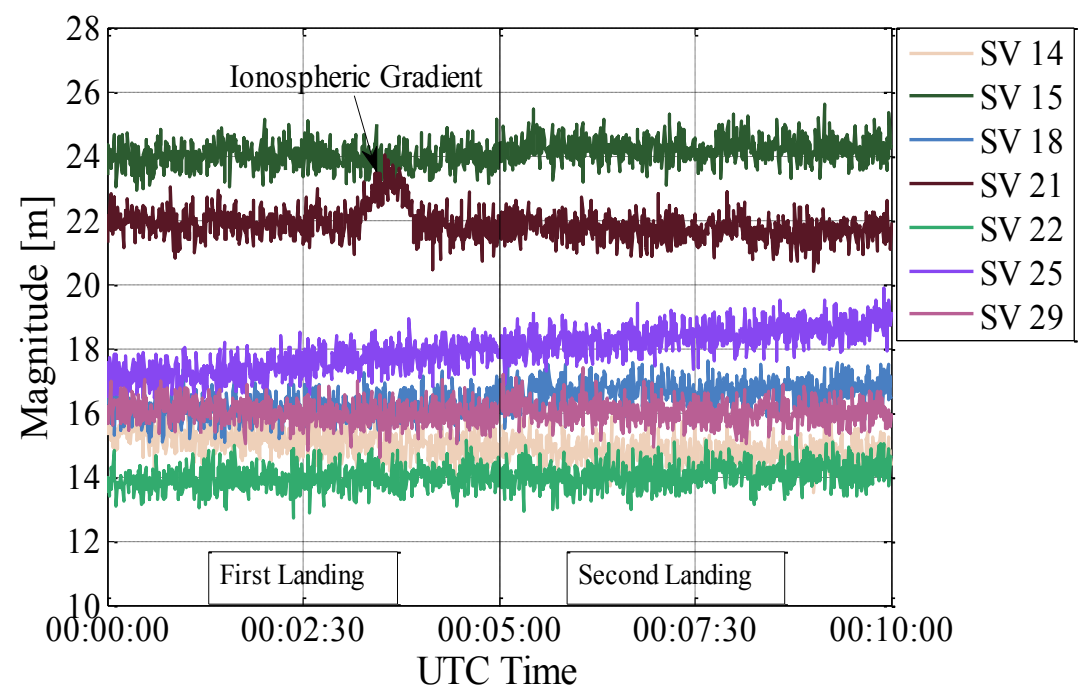

Figure 7.49: Time series of the effects due to ionospheric and tropospheric delays, multipath and random errors on the pseudorange, generated for AZU5346 flight from 00:00:00 UTC to 00:10:00 UTC on 20 December 2014. 


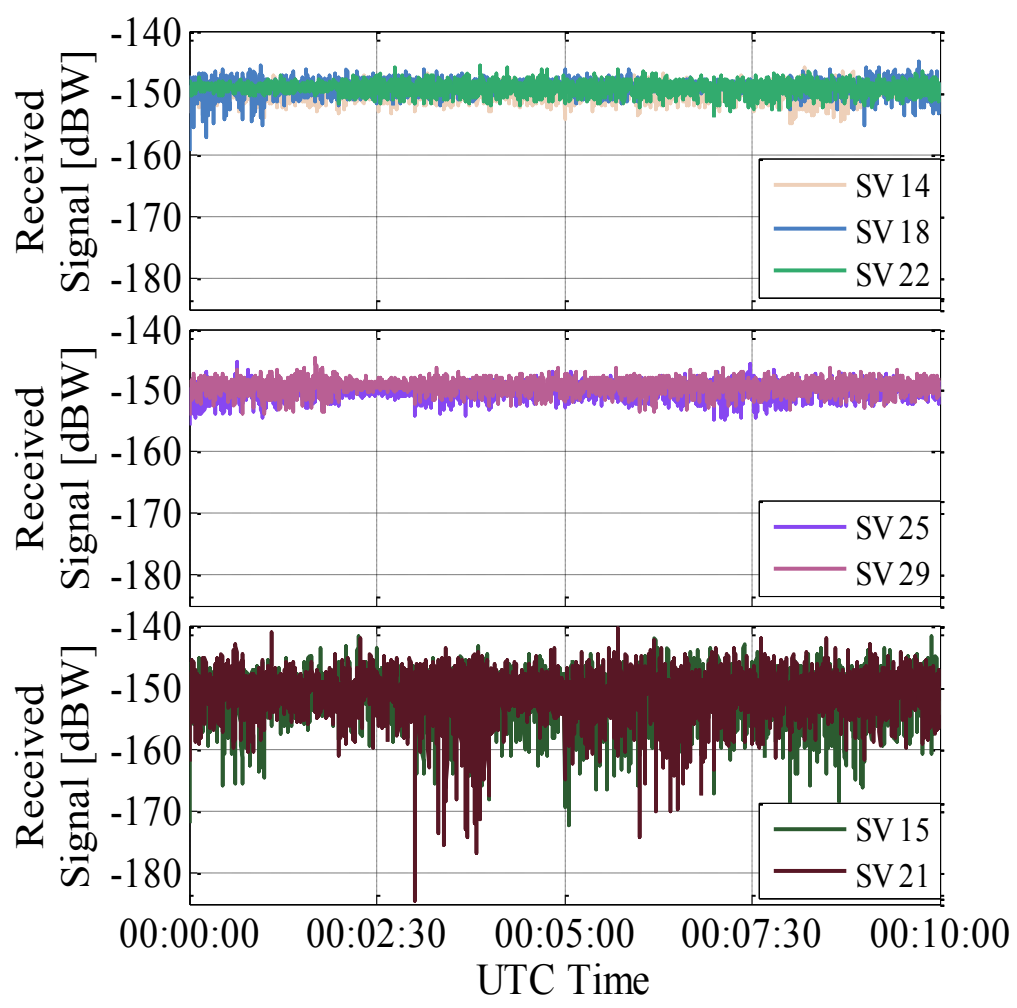

Figure 7.50: Time series of the received signal power, generated for AZU5346 flight from 00:00:00 UTC to 00:10:00 UTC on 20 December 2014, considering the effects due to amplitude scintillation, multipath, and random errors.

The magnitudes of the effects associated with the signal delays of GPS satellites during the period of high solar activity reach $25.5 \mathrm{~m}$, as shown by Figure 7.49. This maximum delay is found for SV 15 , observed from the aircraft position with low elevation angles (approximately $22^{\circ}$ ), as displayed by the skyplot of Figure 7.46. Analyzing Figures 7.7 and 7.49, the differences between the magnitudes of the delay due to the associated effects are evident. These differences, in combination with the IPP positions in Figures 7.4 and 7.46, are taken as an evidence that the southern crest of the EIA, as modeled by IRI and observed by Lin et al. (2007), was located south of Fortaleza and north of Rio de Janeiro. Note that ionospheric spatial gradients estimated between CEEU and CEFT stations were added to the ionospheric delay term of each available channel. For example, SV 21 presents large gradients that reach $244.8 \mathrm{~mm} / \mathrm{km}$, as depicted in Figure 7.46.

Each subplot in Figure 7.50 displays 10-min time series of received signal power generated for the seven tracked GPS satellites. The amplitude scintillation time series were simulated with basis on the $\alpha-\mu$ statistical distribution associated 
with the $\mathrm{S}_{4}$ indices depicted in Figure 7.47. In this scenario, simulated signals from five satellites are consistent with low $\mathrm{S}_{4}$ indices $\left(\mathrm{S}_{4} \leq 0.3\right)$ and from two satellites are consistent with average to high $\mathrm{S}_{4}$ indices $\left(0.4<\mathrm{S}_{4} \leq 0.7\right)$. The top and central panels of Figure 7.50 show that the magnitude of shallow fades are very similar for the five GPS satellite signals with $\mathrm{S}_{4} \leq 0.3$. Deep fades are observed in the lower panel of Figure 7.50, particularly for the SV21 signal. The 10-min time series of the received signal power simulated in Figure 7.50 present shallower scintillation fadings in comparison with the ones generated for an aircraft located near the Rio de Janeiro Airport during a period with high solar activity, displayed in Figure 7.9.

Similarly, time series of pseudorange, carrier phase and received signal power were generated for the four GBAS receivers located at Fortaleza Airport (depicted in Figure 7.44) during the present time interval. In sequence, the GBAS monitor performed the same tests and generated differential corrections, as discussed in more detail in Section 7.1.3. The results from the pseudorange corrections $P R_{\operatorname{corr}(n)}(k)$ estimated for each validated GPS satellite in the current study case are shown in Figure 7.51.

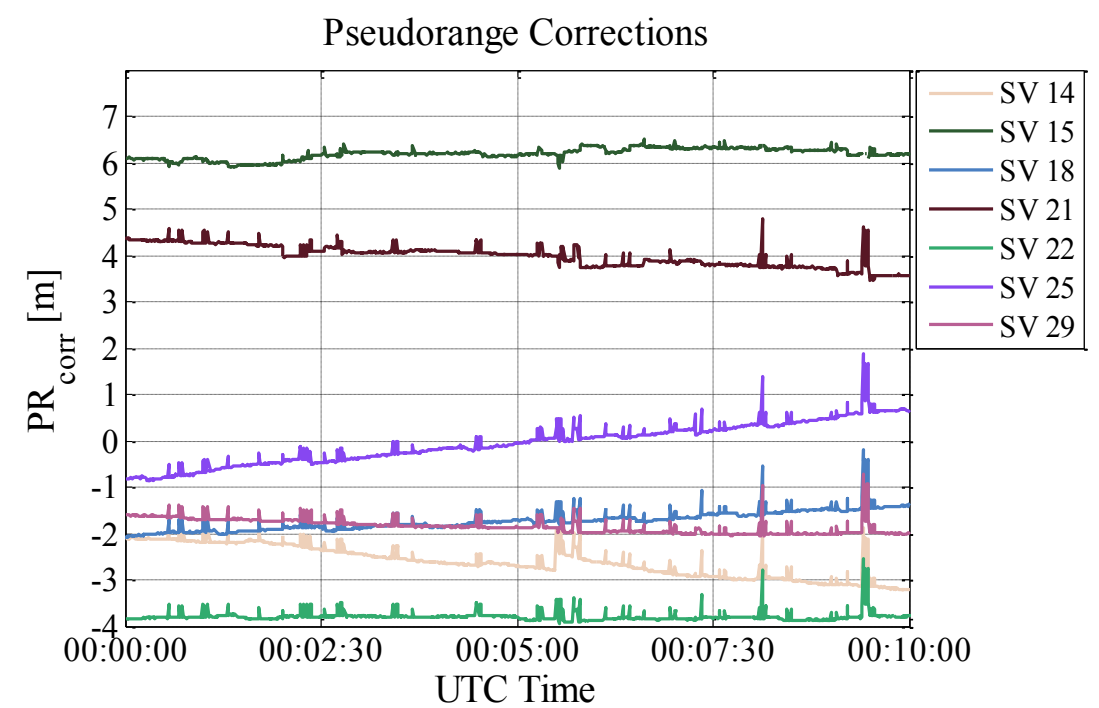

Figure 7.51: Pseudorange corrections estimated by the GBAS during AZU5346 flight from 00:00:00 UTC to 00:10:00 UTC on 20 December 2014.

The pseudorange corrections $P R_{\operatorname{corr}(n)}(k)$ for SV 15 displayed in Figure 7.51 for the Fortaleza environment are larger than those estimated for SV 16 in Figure 
7.23 for the Rio de Janeiro scenario. Again, these differences are credited to the combination of the IPP positions in Figures 7.4, 7.10 and 7.46 and to the position of the southern crest of the EIA. Indeed, SV 15 presents a differential correction of 6.5 meters at 00:07:05 UTC. It is noted that, since the ionosphere is not as perturbed by gradients as that over the Rio the Janeiro Airport, the present differential corrections do not display the previously observed large fluctuations.

Next, the aircraft positions are estimated based on uncorrected and GBAScorrected pseudoranges. The positioning errors are estimated in NED coordinates, using the aircraft path positions as references. The results are shown in the left and right panels of Figure 7.52, respectively.
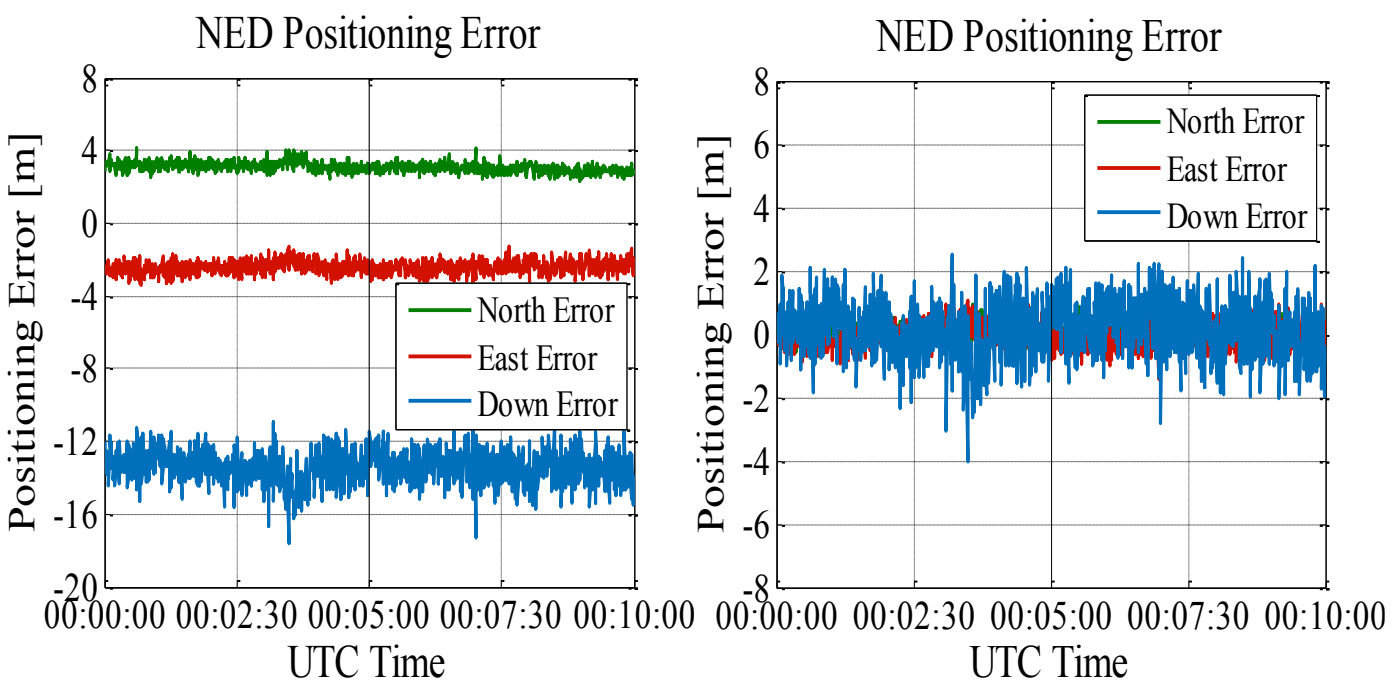

Figure 7.52: NED positioning errors based on uncorrected (Left) and GBAScorrected pseudoranges (Right) during AZU5346 flight from 00:00:00 UTC to 00:10:00 UTC on 20 December 2014.

The aircraft positioning based on uncorrected pseudoranges presents large errors that reached $17.6 \mathrm{~m}$ for the down coordinate, as depicted in the left panel of Figure 7.52. After the GBAS corrections, the same error decreased to $4 \mathrm{~m}$ for the down component as shown by the right panel of Figure 7.52. This maximum error was estimated during the occurrence of large gradients in the first landing, which occurred between 03:12 UTC to 03:53 UTC.

Horizontal and vertical positioning errors are estimated based on the generated observables and corrected pseudoranges, as shown in Figures 7.53 and 7.54, respectively. Additionally, horizontal and vertical protection levels were 
estimated from 00:00:00 UTC to 00:10:00 UTC on 20 December 2014 based on the simulation system parameters described in Table 7.9. The results are represented by the green curves in Figures 7.53 and 7.54. In the present scenario, a sigma vertical ionospheric gradient $\sigma_{\text {vert_iono_grad }}=12 \mathrm{~mm} / \mathrm{km}$ was adopted, according to the values estimated from the Station-Pair method between the CEEU and CEFT RBMC network stations on 20 December 2014.

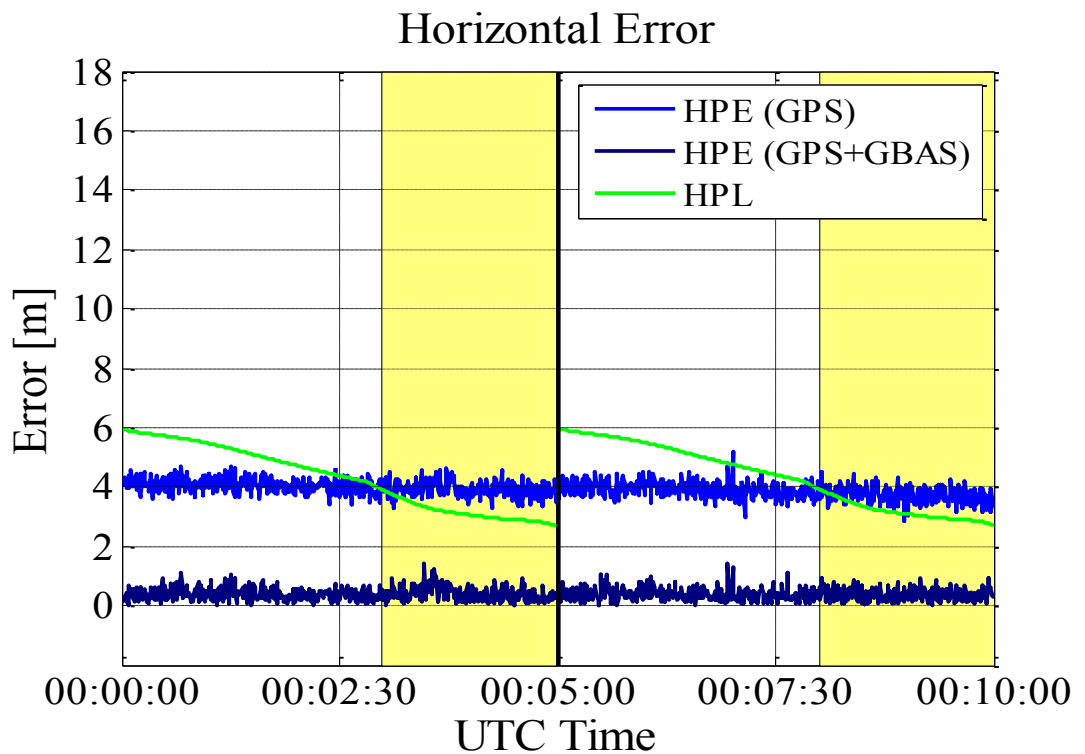

Figure 7.53: Horizontal positioning error and horizontal protection level for AZU5346 flight from 00:00:00 UTC to 00:10:00 UTC on 20 December 2014.

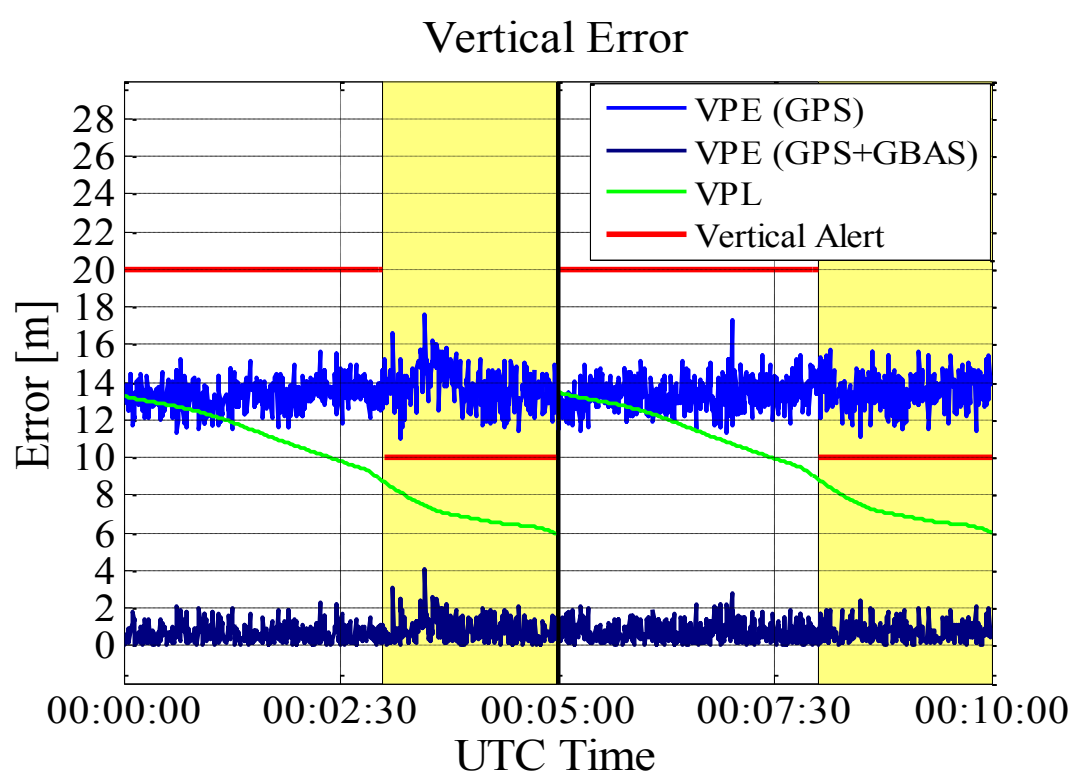

Figure 7.54: Vertical positioning error and vertical protection level for AZU5346 flight from 00:00:00 UTC to 00:10:00 UTC on 20 December 2014. 
For the horizontal component, Figure 7.53 shows that the HPE estimated using uncorrected pseudoranges (blue line) present larger values than the HPL (green line). On the other hand, the corrected observables (dark blue line) present lower values than the HPL during the time intervals for both landings. Similarly, the estimated values for HPL and HPE do not exceed the horizontal alert limit (HAL $=40 \mathrm{~m}$ ) for CAT-I and APV. To display HPE and HPL with an expanded vertical scale, HAL is not represented in Figure 7.53.

For the vertical component, Figure 7.54 shows that the VPE estimated using uncorrected pseudorange (blue line) exceeds the VPL (green line) during most of the time. It is for this reason that corrections are necessary. On the other hand, values estimated for VPE using GBAS-corrected pseudoranges (dark blue) do not exceed the VPL values. In addition, VPL and VPE do not exceed the constant value of $10 \mathrm{~m}$ (red line) of the vertical alert limit (VAL). Therefore, the following inequality holds: VPE $<$ VPL $<$ VAL.

Therefore, the GBAS pseudorange corrections reduced the horizontal and vertical errors between approximately $68 \%$ and $97 \%$, as shown in Figures 7.53 and 7.54 , respectively.

This study case presents results from one scenario with a medium-strong ionospheric impact, represented by spatial gradients and scintillation. For this reason, statistical parameters were estimated to evaluate the accuracy for CAT-I and APV in this unfavorable scenario, as Table 7.12 indicates.

Table 7.12: Accuracy of precision approaches.

\begin{tabular}{|c|c|c|c|c|c|c|}
\hline & \multicolumn{2}{|c|}{ Vertical Positioning Error } & \multicolumn{3}{c|}{ Horizontal Positioning Error } \\
\cline { 2 - 7 } Positioning & $\begin{array}{c}\text { Me an } \\
{[\mathrm{m}]}\end{array}$ & $\begin{array}{c}\text { Standard } \\
\text { de viation } \\
{[\mathrm{m}]}\end{array}$ & $\begin{array}{c}\text { Accuracy } \\
\mathbf{9 5 \%}[\mathbf{m}]\end{array}$ & $\begin{array}{c}\text { Me an } \\
{[\mathrm{m}]}\end{array}$ & $\begin{array}{c}\text { Standard } \\
\text { de viation } \\
{[\mathrm{m}]}\end{array}$ & $\begin{array}{c}\text { Accuracy } \\
\mathbf{9 5 \%}[\mathbf{m}]\end{array}$ \\
\hline $\begin{array}{c}\text { Corrected } \\
\text { (GPS+GBAS) } \\
\text { CAT-I }\end{array}$ & 0.80 & 0.61 & 1.90 & 0.38 & 0.22 & 0.79 \\
\hline $\begin{array}{c}\text { Corrected } \\
\text { (GPS+GBAS) } \\
\text { APV }\end{array}$ & 0.72 & 0.55 & 1.78 & 0.40 & 0.23 & 0.77 \\
\hline
\end{tabular}


The accuracy requirement for GBAS is expressed as a 95th-percentile bound on navigation system error (NSE). The estimated values for this scenario and time interval for the vertical component were 1.90 and $1.78 \mathrm{~m}$ for CAT-I and APV, respectively, while the minimum requirements for GBAS CAT-I and APV are $4 \mathrm{~m}$ and $8 \mathrm{~m}$, respectively (Pullen, 2017). For the horizontal component, the 95\% accuracies were $0.79 \mathrm{~m}$ and $0.77 \mathrm{~m}$ for CAT-I and APV, respectively, while the minimum requirement for GBAS CAT-I and APV is $16 \mathrm{~m}$. In this case, the vertical and horizontal components do not exceed the minimum requirement, influenced by the spatial ionospheric gradient. This exemplifies how spatial gradients (for example, $244.8 \mathrm{~mm} / \mathrm{km}$ ) degrade positioning accuracy. In the present case, they are within the tolerable range for GBAS operation.

The system performances in the horizontal and vertical component at the Fortaleza Airport are shown in the integrity plots displayed in Figures 7.55 and 7.56 , respectively.

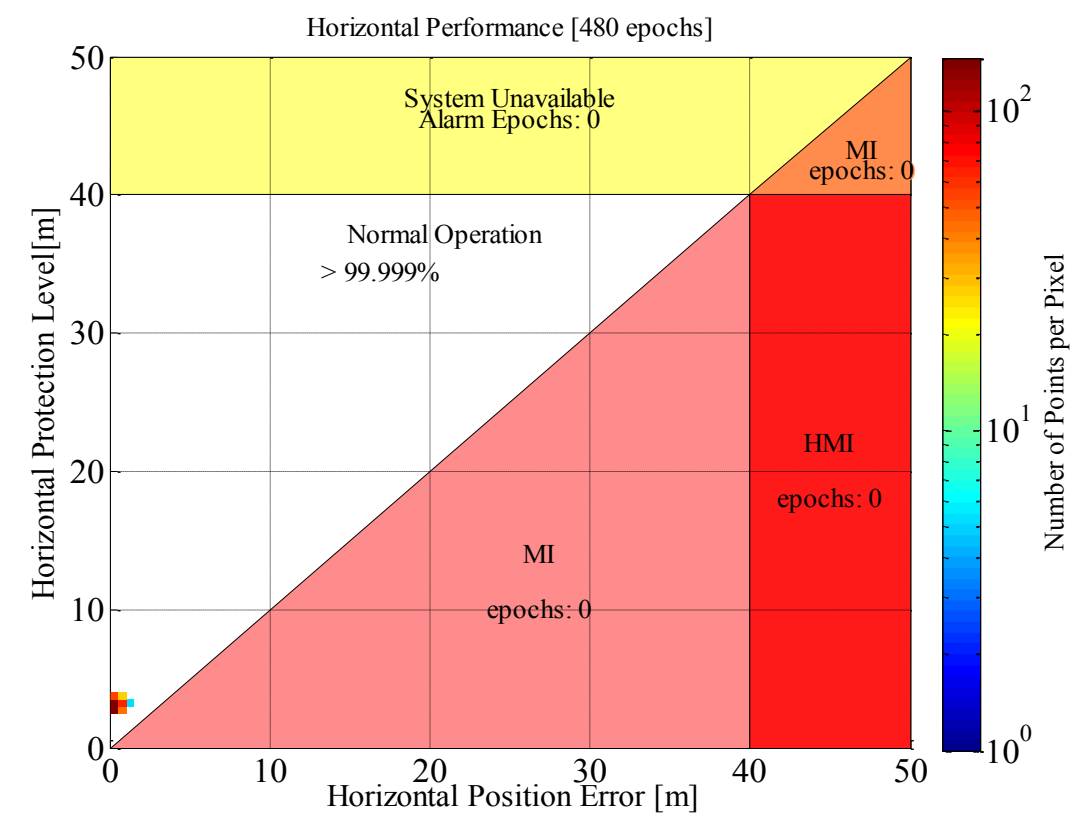

Figure 7.55: Integrity plot of the horizontal performance of AZU5346 flight for CAT-I from 00:00:00 UTC to 00:10:00 UTC on 20 December 2014. 


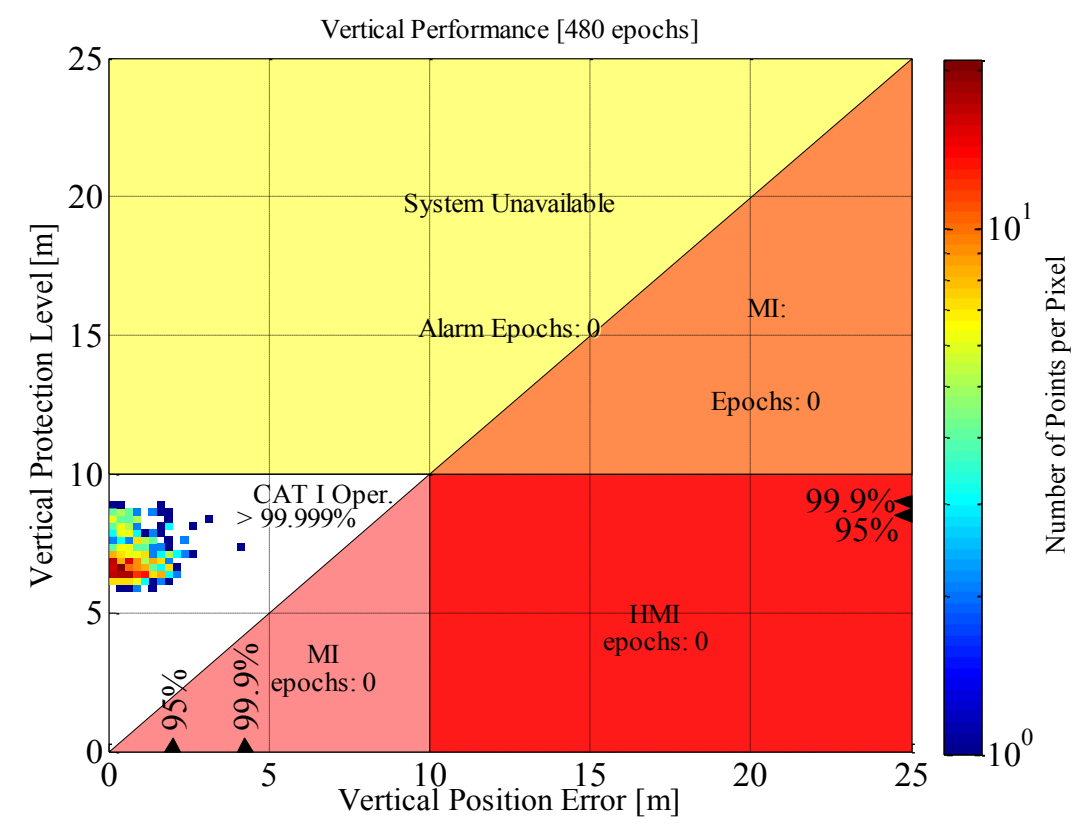

Figure 7.56: Integrity plot of the vertical performance of AZU5346 flight for

CAT-I from 00:00:00 UTC to 00:10:00 UTC on 20 December 2014.

For the horizontal component, Figure 7.55 indicates that all the pairs (HPE, HPL) are located in the Normal Operation region, above the diagonal line HPL = HPE and below the horizontal line HAL $=40 \mathrm{~m}$, even during the intervals of spatial gradients. This plot indicates that the percentage of operation in CAT-I is higher than $99.999 \%$, due to all the 480 epochs corresponding to 2 min in 2 approaches within the operation area for CAT-I. Systems having this property are conservative.

For the vertical component, Figure 7.56 shows that results from all 480 epochs are within the Normal Operation area for CAT-I. The VPE did not exceed the VAL and VPL, and the VPL values are smaller than the VAL $=10 \mathrm{~m}$. It confirms the GBAS availability for the precision approach category at the Fortaleza Airport during an interval of time with high solar activity.

To summarize, the Fortaleza Airport presents low accuracy degradation and it does not lose the integrity condition during periods of high solar activity, indicating the availability of the GBAS. 


\section{Conclusion and Future Work}

\subsection{Conclusion}

The study of propagation and degradation of the Signal in Space (SIS) due to ionospheric effects in equatorial and low latitudes is relevant, to provide better understanding of the medium and its impacts on satellite communication and positioning systems. The ionosphere introduces delays and fadings in the SIS. Chapter 3 presented the methodology used to estimate the vertical TEC, ionospheric delay and ionospheric gradients. Then, this methodology was developed using C, Fortran and MATLAB computational programs to estimate the vTEC based on dual-frequency GPS data from the Rede Brasileira de Monitoramento Continuo. The vTEC was associated to $400-\mathrm{km}$ ionospheric pierce point (IPP) based on the positions of RBMC stations and GPS satellites. The satellite positions were extracted from the Precise Orbit Files computed by the International GNSS Service (IGS). The ionospheric behavior over the Brazilian airspace was studied during the years 2002, 2008 and 2013.

This research presented statistical distributions of residuals estimated between corresponding vTEC values calculated from RBMC data and provided by the International Reference Ionosphere (IRI 2016) model. This analysis considered different combinations of ranges the following geophysical parameters: solar activity, geomagnetic activity; geomagnetic latitude; local time; and season, resulting in 72 statistical distributions.

Some of the distributions presented large residuals. The statistical distribution associated to the geomagnetic latitude of IPP: $\left(-4^{\circ},-12^{\circ}\right)$ or $\left(+4^{\circ}\right.$, $+12^{\circ}$ ); local time: 21:30 LT - 01:30 LT; season: April - August; solar activity (F10.7 index): F10.7 > 150; geomagnetic activity (Kp index): Kp $>4$ presented a mean residual equal to 16.81 TECU. This results indicate that the vTEC obtained using the IRI model underestimate that estimated using RBMC measurements. On 
the other hand, the statistical distribution associated to the geomagnetic latitude of IPP: $\left(-12^{\circ},-22^{\circ}\right)$; local time: 18:30 LT - 21:30 LT; season: September - March; Solar activity: F10.7 $>150$; geomagnetic activity: $0<\mathrm{Kp} \leq 4$ ) presented the mean residual -8.11 TECU, indicating the opposite relationship.

In Chapter 4, ionospheric gradients were estimated based on the time-step and station-pair methods, using the vTEC results from Chapter 3. The gradients frequently exceeded $400 \mathrm{~mm} / \mathrm{km}$ in periods of high solar activity. Occasionally, they even exceeded $600 \mathrm{~mm} / \mathrm{km}$. All of the detected threatening gradients occurred between the post-sunset and early post-midnight hours. The maximum estimated gradients are equal to $588.79 \mathrm{~mm} / \mathrm{km}, 974.23 \mathrm{~mm} / \mathrm{km}$ and 970.71 $\mathrm{mm} / \mathrm{km}$, for IMPZ, BRAZ and RIOD station, respectively, obtained from the application of the time-step method to 2013 RBMC data (year of high solar activity, with periods of equally intense geomagnetic activity). On the other hand, the application of the station-pair method to 2013 RBMC data estimated maximum gradients equal to $939.90 \mathrm{~mm} / \mathrm{km}, 334.40 \mathrm{~mm} / \mathrm{km}$ for pair, and 908.33 $\mathrm{mm} / \mathrm{km}$ for the ONRJ-RIOD, CEEU-CEFT, and SSA1-SAVO pairs, respectively.

For completeness, the GPS SIS was developed using a statistical model considering IRI outputs and residuals from the RBMC estimates, in combination with ionospheric scintillation modules, tropospheric delays, cycle ambiguity, receiver clock, multipath, and random errors, as described in Chapter 5. For instance, the amplitude scintillation time series simulated for the Fortaleza station display shallower fadings in comparison to the values generated for the Rio de Janeiro station for most of the satellites. This is mostly due to the existing perturbations in the ionosphere under different latitudes in relation to the geomagnetic equator, which tend to yield smaller values of $\mathrm{S}_{4}$ at the former.

The procedure implemented to simulate the GBAS Ground Facility was presented in Chapter 6, which apply a set of monitoring algorithms to detect a varied array of possible failures in the GPS SIS and generates corrections.

In Chapter 7, the GPS SIS and GBAS processing were simulated at the Rio de Janeiro and Fortaleza Airports, generating corrections for the last section of 
TAM/JJ3839 flight and AZU5346 flight operated by LATAM and AZUL airlines, respectively.

The aircraft positions from TAM/JJ3839 flight estimated in approaches to the Rio de Janeiro Airport presented degradation of the accuracy and loss of the integrity condition during periods of high solar activity, reducing the availability of the GBAS. The existence of large gradients (approximately $604.5 \mathrm{~mm} / \mathrm{km}$ ) and strong ionospheric scintillation $\left(0.6 \leq \mathrm{S}_{4} \leq 0.9\right)$ affected the availability during the interval of time analyzed considering the CAT-I approach. On the other hand, the aircraft positions estimated during periods of low solar activity demonstrated high accuracy and satisfy the integrity condition, indicating a high availability of the GBAS due to the small gradients and weak ionospheric scintillation $\left(\mathrm{S}_{4} \leq 0.3\right)$.

The aircraft positions from AZU5346 flight estimated in approaches to Fortaleza Airport presented low degradation of the accuracy but satisfy the integrity condition during periods of high solar activity, indicating the availability of the GBAS.

\subsection{Future work}

As mentioned in Chapter 7, estimations of the protection levels depend heavily on sigma vertical ionospheric gradients. Future researches should focus on calculating the sigma vertical ionospheric gradient using an inflation factor. This factor can be estimated using the statistical models based on the gradients and their occurrence as a function of the elevation angle, as well as on the geomagnetic latitude for each airport.

Additionally, it would be interesting to revise the formulation developed to estimate the aircraft position by including the weighting matrix W (Kaplan and Hegarty, 1996; Silva, 2020) and comparing corresponding results.

Future works could assess and statistically characterize the results of the Quality Monitor algorithms based on geophysical parameters and IPP geomagnetic latitudes to mitigate the ionospheric effects on positioning using 
GBAS. Also, this statistical results should consider inflation factors to estimate the threshold for each test.

There still are many interesting topics open to research on GBAS, ranging from the development of a full concept of operation simultaneously using multiple constellations (GLONASS, Galileo, BeiDou, and etc.) along with the required availability concepts to achieve higher integrity and accuracy corrections in equatorial and low latitude regions.

Further studies of the GBAS performance in equatorial and low-latitude ionospheric effects could include the new GPS signal (L5 at $1176.45 \mathrm{MHz}$ ). This frequency diversity will be used to improve the performance of GPS itself, as well as its applications, including the GBAS. The dual frequency L1/L5 can be used to estimate the ionospheric delay accurately in GBAS receivers, in addition to maintain a higher availability. 


\section{Bibliography}

AARONS, J. Global morphology of ionospheric scintillation. Proceedings of the IEEE, vol. 70. pp. 360-378, 1982.

ABDU, M. A. Equatorial ionosphere-thermosphere system: electrodynamics and irregularities. Advances in Space Research, vol. 35, pp. 771-787, 2005.

ABRAMOWITZ, M.; STEGUN, I. Handbook of Mathematical functions. National Bureau of Standards Applied Mathematics, USA, 1972.

ALVES, D. B. M.; MONICO, J. F. G.; DALBELO, L. F. A.; SHIMABUKURO, M. H. First Brazilian real time network DGPS through the internet: development, appli-cation and availability analyses. Journal of Geodetic Science, vol. 1, pp. 1-7, 2011.

BALANIS, C. A. Advanced Engineering Electromagnetics. Wiley, New York, USA, 1989.

BANOLA, S.; PATHAN, B. M.; RAO, D. R. K.; CHANDRA. H. Spectral characteristics of scintillations producing ionospheric irregularities in the Indian region. Earth, Planets and Space, vol. 57, pp. 47-59, 2005.

BASU, SU.; BASU, SA. Equatorial scintillations - A review. Journal of Atmospheric and Solar-Terrestrial Physics, vol. 43, pp. 473-489, 1981.

BASU, SU.; WHITNEY, H. E. The temporal structure of intensity scintillation near the magnetic equator. Radio Science, vol. 18, pp. 263-271, 1983.

BASU, S.; GROVES, K. M.; QUINN, J.; DOHERTY, P. H. A comparison of TEC fluctuations and scintillations at Ascension Island. Journal Atmospheric Solar-Terrestrial Physics, vol. 61 (16), pp.1219-1226, 1999.

BILITZA, D.; ALTADILL, D.; ZHANG, Y.; MERTENS, C.; TRUHLIK, V.; RICHARDS, P.; MCKINNELL, L.; REINISCH, B. The International Reference Ionosphere 2012- a model of international collaboration. Journal of Space Weather and Space Climate, vol. A07, pp. A071-A710, 2014. 
BLEWITT, G. An automatic editing algorithm for GPS Data. Geophysical Research Letters, vol.17, pp. 199-202, 1990.

BOEING. Determining the vertical alert limit requirements for a level of GBAS service that is appropriate to support CATII/III operations. October, 2005.

BECKMANN P.; SPIZZICHINO, A. The Scattering of Electromagnetic Waves from Rough Surfaces. Pergamon Press. Oxford, United Kingdom, 1963.

BENIGUEL, Y.; ADAM, J. P. Effects of Scintillations in GNSS Operation. Springer Netherlands, pp. 203-2016, 2007.

BYUN, S. H.; HAJJ, G. A.; YOUNG, L. E. Development and application of GPS signal multipath simulator. Radio Science, vol. 37(6), pp. 10.1-10.23, 2002.

BRIGGS B. H.; PARKIN, I. A. On the variation of radio star and satellite scintillation with zenith angle. Journal of Atmospheric and Terrestrial Physics, vol. 25, pp. 339-366, 1963.

CANSO (Civil Air Navigation Services Organization). Demystifying GNSS, January 2005.

CARRANO, C. S.; GROVES, K. M.; GRIFFIN, J. M. Empirical characterization and modelling of GPS positioning errors due to ionospheric scintillation. Ionospheric Symposium, Alexandria, Virginia, USA, 2005.

CARRANO, C. S.; GROVES, K. M. Ionospheric data processing and analysis. Workshop on Satellite Navigation Science and Technology for Africa, 2009.

CIRAOLO, L.; AZPILICUETA, F.; BRUNINI, C.; MEZA, A. Calibration errors on experimental slant total electron content (TEC) determined with GPS. Journal of Geodesy, vol. 81, pp. 111-120, 2007.

CONKER, R. S.; EL-ARINI, M. B.; HEGARTY, C. J.; HSIAO, T. Modelling the effects of ionospheric scintillation on GPS/ Satellite-based Augmentation System availability. Radio Science, vol. 38, pp. 1-23, 2003.

CRANE, R. K. Ionospheric scintillation. Proceedings of the IEEE, vol. 65(2) pp. 180-199, 1977. 
DACH, R.; HUGENTOBLER, U.; FRIDEZ, P.; MEINDL, M. Bernese Software Version 5.0. Astronomical Institute, University of Bern, 2007.

DAI, Z. MATLAB software for GPS cycle slip processing. GPS Solutions, vol. 16, pp. 267-272, 2012.

DATTA-BARUA, S.; WALTER, T.; BLANCH, J.; ENGE, P. Bounding higher order ionosphere errors for the dual frequency GPS user. Proceedings of ION GNSS, pp. 1377-1392, 2006.

DATTA-BARUA, S.; WALTER, T.; PULlEM, S.; LUO, M.; ENGE, P. Using WAAS ionospheric data to estimate LAAS short baseline gradients. Proceedings of ION GNSS 2002, pp. 523 530, 2002.

DATTA-BARUA, S. Ionospheric Threats to the Integrity of Airborne GPS Users. PhD thesis, Department of Aeronautics and Astronautics, Stanford University, USA. pp.144, 2008.

DAVIES, K. Ionospheric Radio. The Institution of Electrical Engineers, 1990.

DE PAUlA, E. R.; KHERANI, E. A.; BATISTA, I. S.; SOBRAL, J. H. A; KANTOR, I. J.; TAKAHASHI, H.; REZENDE, L. F. C.; MUELLA, M. T. A. H.; RODRIGUES, F. S.; KINTNER, P. M.; LEDVINA B. M.; MITCHELL, C.; GROVES K. M. Characteristics of the ionospheric F-region plasma instabilities over Brazilian longitudinal sector. Indian Journal of radio and Space Physics, vol. 36, pp. 268-277, 2007.

EL-GIZAWY, M. L. Development of an ionosphere monitoring technique using GPS measurements for high latitude GPS users. M.Sc. Thesis, Department of Geomatics Engineering, The University of Calgary, Calgary, pp. $161,2003$.

EUROCONTROL. WGS 84 Implementation Manual. Version 2.4, 1998.

FAA. U.S. Federal Aviation Administration. Satellite Navigation - Wide Area Augmentation System (WAAS), 2018a. Available Website in https://www.faa.gov/about/office_org/headquarters_offices/ato/service_units/tech ops/navservices/gnss/waas/

FAA U.S. Federal Aviation Administration. Specification: Performance Type One Local Area Augmentation System Ground Facility. FAA-E-2937A, 2002. 
FERREIRA, P. A. Operational Evaluation of a GBAS System. M.Sc. thesis, Instituto Superior Tecnico, Universidade Tecnica de Lisboa, Portugal, pp.96, 2007.

FORTE, B. On the relationship between the geometrical control of scintillation indices and the data detrending problems observed at high latitudes. Annals of Geophysics, vol. 50(6), pp. 699- 706, 2007.

HARGREAVES, J. K. The Upper Atmosphere and Solar-Terrestrial Relations. Cambridge University Press, 1992.

HOFMANN-WELLENHOF, B.; LICHTENEGGER, H.; WASLE, E. GNSS Global Navigation Satellite Systems - GPS, GLONASS, Galileo \& more. SpringerVerlag Wien, 2008.

HUMPHREYS, T. E.; PSIAKI, M. L.; HINKS, J. C.; O'HANLON, B.; KINTNER, P. Simulating ionosphere-induced scintillation for testing GPS receiver phase tracking loops. IEEE Journal of Selected Topics in Signal Processing, vol. 3(4), 2009.

ICAO. Annex 10 to the Convention on International Civil Aviation. Aeronautical Telecommunications Radio Navigation Aids, vol. 2, 2001.

ICAO. Manual on Required Navigation Performance, 2nd Edition, 1999.

ICEA. Instituto de Controle do Espaço Aéreo. Relatório técnico sobre análise de dados ionosféricos e dados de desempenho do sistema GBAS instalado no Aeroporto do Galeão no Rio de Janeiro. Relatorio 001/PCCt/2013, 2013.

ITU-R Recommendation P.453-13, The radio refractive index: Its formula and refractive data. International Telecommunication Union, Geneva, 2017.

JAKES, W. C. Microwave Mobile Communications. IEEE Press, New York, 1974.

KAPLAN, E. D.; HEGARTY, C. J. Understanding GPS Principles and Applications. Artech House, Boston, 1996.

KELley, M. C. The Earth's Ionosphere: Plasma Physics and Electrodynamics. 2nd edition, Academic Press, 2009. 
KENPANKHO, P.; WATTHANASANGMECHAI, K.; SUPNITHI, P.; TSUGAWA, T.; MARUYAMA, T. Comparison of GPS TEC measurements with IRI TEC prediction at the Equatorial Latitude Station, Chumphon, Thailand. Earth Planets Space, vol. 63, pp. 365-370, 2011.

KINTNER, P. M.; LEDVINA, B. M.; DE PAULA. E. R. GPS and ionospheric scintillations. Space Weather, vol. 5(9), pp. 1-23, 2007.

KLEUSBERG, A.; TEUNISSEN, P.J.G. GPS for Geodesy. 2nd Edition, Springer, 1998.

KOENIG, M. Optimizing the Decision Rule of GPS Integrity Monitoring System for Improved Availability. PhD. thesis Department of Mechanical Engineering, Stanford University, USA, pp. 224, 2010.

LANGLEY, R. B. GPS Receivers and the Observables, GPS for Geodesy. Springer, Berlin, Heidelberg, 1998.

LAAS KTA Group. FAA LAAS Ground Facility (LGF) Functions. Unpublished Manuscript, vol. 2.4, 1998.

LEE, J.; PULLEN, S.; XIE, G.; ENGE, P. LAAS sigma monitor analysis and failure-test verification. Proceedings of the $57^{\text {th }}$ Annual Meeting of The Institute of Navigation, pp. 694-704, 2001.

LEE, J. GPS-Based Aircraft Landing Systems with Enhanced Performance: Beyond Accuracy. PhD. thesis Department of Aeronautics and Astronautics, Stanford University, USA, pp. 146, 2005.

LIN, C. H.; LIU, J. Y.; FANG, T. W.; CHANG, P. Y.; TSAI, H. F.; CHEN, C. H.; HSIAO, C. C. Motions of the equatorial ionization anomaly crests imaged by FORMOSAT-3/COSMIC, Geophysical Research Letters, vol. 34, L19101, 2007.

LIU, Z.; GAO, Y. Ionospheric TEC predictions over a local area GPS reference network. GPS Solutions, vol. 8, pp. 23-29, 2003.

MA, G.; MARUYAMA, T. Derivation of TEC and estimation of instrumental biases from GEONET in Japan. Annales Geophysicae, vol. 21, pp. 2083-2093, 2003. 
MANNUCCI, A. J.; WILSON, B. D.; YUAN, D. N.; LINDQWISTER, U. J.; RUNGE, T. F. A global mapping technique for GPS-derived ionospheric total electron content measurements. Radio Science, vol. 33, pp. 565-582, 1998.

MCGRAW G., MURPHY, T.; BRENNER, M.; PULLEN, S. Development of the LAAS accuracy models. Proceedings of the ION GPS 2000, pp. 1212-1223, 2000 .

MCNAMARA, L. F. Radio Amateurs Guide to the Ionosphere. Krieger Publishing Company, 1994.

MILLER, A. R.; BROWN, R. M.; VEGH, E. New derivation for the roughsurface reflection coefficient and for the distribution of the sea-wave elevations. IEEE Proceedings, vol. 131(2), pp. 114-116, 1984.

MIRUS TECHNOLOGY. Report: Effects of Low Latitude Ionospheric Activity On Global Navigation Satellite Systems (GNSS). 2015.

Mitelman, A.; Phelts, R.E.; AKOS, D.; PUlLEN, S.; ENGE, P. A realtime signal quality monitor for GPS augmentation systems. Proceedings of ION GPS 2000, pp. 862-871, 2000.

MOHINDER, S. G.; LAWRENCE R. W.; ANGUS P. A. Global Positioning Systems, Inertial Navigation and Integration. John Wiley \& Sons, Inc., 2007.

MONICO, J. F. G.; ROFATTO, V. F.; MENDOCA, M. A. M. Introdução e aplicação da diluição da precisão da ambiguidade GNSS-ADOP. Boletim de Ciências Geodésicas, vol. 20, pp. 279-299, 2014.

MONICO, J. F. G. Posicionamento pelo GNSS: Descrição, Fundamentos e Aplicações. 2. ed. Editora da UNESP, 2008.

MORAES, A. O. Advances in Statistical Modeling of Ionospheric Scintillation. PhD. thesis Department of Graduate Studies of the Aeronautics Institute of Technology, Aeronautical Institute of Technology, Brazil, pp. 141, 2013.

MORAES, A. O.; COSTA, E.; DE PAULA, E. R.; PERELLA, W. J.; GALERA, J. F. Extended ionospheric amplitude scintillation model for GPS receivers. Radio Science, vol. 49(5), pp. 315-329, 2014. 
MORAES, A. O.; COSTA, E.; ABDU, M. A.; RODRIGUES, F.; DE PAULA, E. R.; OLIVEIRA, K.; PERELLA, W. J. The variability of low-latitude ionospheric amplitude and phase scintillation detected by a triple-frequency GPS receiver. Radio Science, vol. 52(4), pp. 439-460, 2017.

MORAES, A. O.; VANI, B. C.; COSTA, E.; SOUSASANTOS, J.; ABDU, M. A.; RODRIGUES, F.; GLADEK Y. C.; DE OLIVEIRA, C. B.; GALERA, J. F. Ionospheric scintillation fading coefficients for the GPS L1, L2, and L5 frequencies. Radio Science, vol. 53(9), pp. 1165-1174, 2018.

NIELL, A. Global mapping functions for the atmosphere delay at radio wavelengths. Journal of Geophysical Research, vol. 101, pp. 3227-3246, 1996.

OTSUKA, Y.; OGAWA, T.; SAITO, A.; TSUGAWA, T.; FUKAO, S.; MIYAZAKI, S. A new technique for mapping of total electron content using GPS network in Japan. Earth Planets Space, vol. 54, pp. 54-63, 2002.

PARKINSON, B. W.; GILBERT S. W. NAVSTAR: Global Positioning System - ten years later. Proceedings of the IEEE. vol. 71, pp. 1177-1186, 1983.

PEREIRA, V. A. S. Avaliação e Usabilidade do GBAS no Brasil. PhD. Thesis, Faculdade de Ciências e Tecnologia da Universidade Estadual Paulista "Júlio de Mesquita Filho”, UNESP, Brazil, pp. 307, 2018.

PEREIRA, V. A.; MONICO, J. G.; CAMARGO, P. O. Procedimento geral para o desenvolvimento de modelo de risco ionosférico para GBAS e principais modelos existentes. IV Simpósio Brasileiro de Geomática - SBG2017 - II Jornadas Lusófonas - Ciências e Tecnologias da Informação Geográfica CTIG2017, pp. 563-570, 2017.

PROL, F. S.; DE OLIVEIRA, P. C.; MUELLA, M. T. A. H. Comparative study of methods for calculating Ionospheric Points and describing the GNSS signal path. Boletim de Ciências Geodésicas, vol. 23(4), pp. 669-683, 2017.

PUllen, S.; OPShAUG, G.; HANSEN, A.; WALTER, T.; ENGE, P.; PARKINSON, B. A preliminary study of the effect of ionospheric scintillation on WAAS user availability. Proceedings of ION GPS 1998, pp. 687-699, 1998. 
PULLEN, S.; LUO, M.; XIE, G.; ENGE, P. GBAS validation methodology and test results from the Stanford LAAS Integrity Monitor Testbed. Proceeding of the ION GPS 2000, pp. 1191-1201, 2000.

PULLEN, S.; LEE, J.; LUO, M. Ephemeris protection level equations and monitor algorithms for GBAS. Proceedings of ION GPS 2001, pp. 1738-1749, 2001.

PULLEN, S. Augmented GNSS: Fundamentals and Keys to Integrity and Continuity. ION GNSS 2011 Tutorial, 2011.

PULlEN, S. Ground Based Augmentation System. In: Global Navigation Satellite System. Springer, pp. 905- 932, 2017.

RAY, J. K.; CANNON, M. E.; FENTON, P. GPS code and carrier-phase multipath mitigation using multiantenna system. IEEE Transactions on Aerospace and Electronic Systems, vol. 37(1), 2001.

RIDEOUT, W.; COSTER, A. Automated GPS processing for global total electron content data. GPS Solutions, vol. 10(3), pp. 219-228, 2006.

RINO, C. L. The Theory of Scintillation with Applications in Remote Sensing. Wiley-IEEE Press, 2011.

RINO, C. L.; CARRANO, C. S. The application of numerical simulations in Beacon scintillation analysis and modeling. Radio Science, pp. 1-17, 2011.

RTCA. Minimum Operational Performance Standards for GPS/Local Area Augmentation System Airborne Equipment. 2008.

RUNGRAENGWAJIAKE，S.; SUPNITHI， P.; SAITO， S.; SIANSAWASDI, N.; SAEKOW, A. Ionospheric delay gradient monitoring for GBAS by GPS stations near Suvarnabhumi Airport, Thailand. Radio Science, vol. 50(10), 2015.

SAASTAMOINEN, J. Atmospheric correction for the troposphere and stratosphere in radio ranging of satellites. The Use of Artificial Satellites for Geodesy, Geophysical Monograph Series, vol. 15, pp. 247-251, 1972.

SCHAER, S. Mapping and predicting the Earth's ionosphere using the Global Positioning System. Geodesy Geophysics. Arb. Schweiz, vol. 59, 1999. 
SCHÜLER, T. On Ground-Based GPS Tropospheric Delay Estimation. PhD. thesis Department of Civil Engineering and Surveying, Bun Munich University, Germany, pp. 364, 2001.

SEEBER, G. Satellite Geodesy. 2nd Edition, Walter de Gruyter, 2003.

SEO, J. Overcoming Ionospheric Scintillation for Worldwide GPS Aviation.

$\mathrm{PhD}$. thesis Department of Aeronautics and Astronautics, Stanford University, USA, pp. 154, 2010.

SILVA, W. C. GBAS: fundamentos e análises de operacionalidade em função do sigma VIG. M.Sc. Dissertation Faculdade de Ciências e Tecnologia da Universidade Estadual Paulista "Júlio de Mesquita Filho", UNESP, Brazil, pp. 98, 2020.

SINGLETON, D. G.; LYNCH, G. J. The scintillation of the radio transmissions from Explorer VII-II: Some properties of the scintillation producing irregularities. Journal of Atmospheric and Terrestrial Physics, vol. 24, pp. 363-374, 1962.

SKIDMORE, T.; VAN GRAAS, F. DGPS Ground station integrity monitoring. Proceeding of the ION National Technical Meeting, San Diego, California, USA, 1994.

SURCO, T. M. E.; COSTA, E.; DE PAULA E. R. Estimation and analysis of Differential Code Bias based on GPS data from Brazilian Network. Simpósio Brasileiro de Telecomunicações e Processamento de Sinais SBRT 2019, Brazil, 2019.

TEUNISSEN, P. J. G. The least-squares ambiguity decorrelation adjustment: a method for fast GPS integer ambiguity estimation. Journal of Geodesy, vol. 70(1), pp. 65-82, 1995.

WOODMAN R. F.; LA HOZ C. Radar observations of $\mathbf{F}$ region equatorial irregularities. Journal of Geophysical Research, vol. 8, pp. 5447-5466, 1976.

XIE, G.; PULLEN, S.; LUO, M.; NORMARK, P.; AKOS D.; LEE J.; ENGE P. Integrity design and updated test results for the Stanford LAAS Integrity Monitor Testbed. Proceedings of the $57^{\text {th }}$ Annual Meeting of The Institute of Navigation, pp. $681-693,2001$. 
XIE, G. Optimal On-Airport Monitoring of the Integrity of GPS-Based Landing Systems. PhD. thesis Department of Electrical Engineering, Stanford University, USA, pp. 212, 2004.

XU, G. GPS: Theory, Algorithms and Applications. $2^{\text {nd }}$ edition, Springer, 2007. 


\section{APPENDIX A - Satellite Positions using Keplerian Elements}

In orbital mechanics, the two-body problem predicts the motions of two massive objects, abstractly viewed as point particles. There are many possible formulations for the two-body problem, but the GPS literature generally adopts the classical solution, which uses a particular set of six integrals of motion based on the Keplerian orbital elements (Kaplan and Hegarty, 1996). This Keplerian elements can be used to estimate the position of each GPS satellite at epoch $k$, with respect to the Earth-centered Earth-fixed reference frame (ECEF).

The estimation of the position of each GPS satellite at epoch $k$ is based on the parameters described in Table A.1. and are available in the Navigation files.

Table A.1: Parameters available in the Navigation files.

\begin{tabular}{|c|c|}
\hline Parameter & Definition \\
\hline$t_{o e}$ & Mean motion correction \\
\hline$\Delta_{n}$ & Square root of semi major axis \\
\hline$\sqrt{a}$ & Mean anomaly \\
\hline $\bar{M}_{0}$ & Excentricity \\
\hline$e$ & Argument of the perigee \\
\hline$\omega$ & Amplitude of the cosine correction argument of latitude \\
\hline$C_{u c}$ & Amplitude of the sine correction argument of latitude \\
\hline$C_{u s}$ & Amplitude of the cosine correction to the orbital radius \\
\hline$C_{r c}$ & Amplitude of the sine correction to the orbital radius \\
\hline$C_{r s}$ & Amplitude of the cosine correction to inclination angle \\
\hline$C_{i c}$ & Amplitudes of the sine correction to inclination angle \\
\hline$C_{i s}$ & Inclination angle at time of ephemeris \\
\hline$i_{0}$ & Rate of change of inclination angle \\
\hline$d i / d t$ & Longitude of the ascending mode \\
\hline$\Omega_{0}$ & Rate of change of longitude of the ascending mode \\
\hline$\dot{\Omega}$ & \\
\hline
\end{tabular}


The corrected mean motion $n$ is represented by the following equations

$$
n=\sqrt{\frac{G M}{a^{3}}}+\Delta_{n}
$$

where $G M$ is the gravitational constant $\left(G M=3.986004 \cdot 10^{14} \mathrm{~m}^{3} / \mathrm{s}^{2}\right)$.

The mean anomaly $\bar{M}_{k}$ at the time $k$ from ephemeris can be represented by

$$
\bar{M}_{k}=\bar{M}_{0}+n \cdot \Delta t_{k}
$$

where $\Delta t_{k}$ is the time from the ephemeris epoch, represented by

$$
\Delta t_{k}=t^{s}-t_{o e}
$$

In the above expression, $t^{S}$ is the observation epoch (associated with the index $k$ )

The eccentric anomaly $E_{k}$ can be calculated by

$$
E_{k}=\bar{M}_{k}+e \cdot \sin \left(E_{k}\right)
$$

Equation (A.4) must be solved iteratively. Next, the true anomaly $v_{k}$ can be obtained from the following equations

$$
\begin{gathered}
\operatorname{sen}\left(v_{k}\right)=\frac{\sqrt{1-e^{2}} \cdot \sin \left(E_{k}\right)}{1-e \cdot \cos \left(E_{k}\right)} \\
\cos \left(v_{k}\right)=\frac{\cos \left(E_{k}\right)-e}{1-e \cdot \cos \left(E_{k}\right)}
\end{gathered}
$$

The plane coordinates of the satellite, represented by $X_{p}$ and $Y_{p}$, are calculated using the following formulation. Initially, the corrected latitude argument $u_{k}$; the latitude argument $\Phi_{k}$ and the correction latitude argument $\delta u_{k}$ can be estimated by 


$$
\begin{gathered}
u_{k}=\Phi_{k}+\delta u_{k} \\
\Phi_{k}=v_{k}+\omega \\
\delta u_{k}=C_{u c} \cdot \cos \left(2 \Phi_{k}\right)+C_{u s} \cdot \sin \left(2 \Phi_{k}\right)
\end{gathered}
$$

In sequence, the corrected radius $r_{k}$ and the radius correction $\delta r_{k}$ can be expresses as

$$
\begin{gathered}
r_{k}=a \cdot\left(1-e \cdot \cos E_{k}\right)+\delta r_{k} \\
\delta r_{k}=C_{r c} \cdot \cos \left(2 \Phi_{k}\right)+C_{r s} \cdot \sin \left(2 \Phi_{k}\right)
\end{gathered}
$$

After that, it is possible to calculate the plane coordinates of the satellite $X_{p}$ and $Y_{p}$ using the equation (A.7) and (A.10)

$$
\begin{gathered}
X_{p}=r_{k} \cdot \cos \left(u_{k}\right) \\
Y_{p}=r_{k} \cdot \sin \left(u_{k}\right)
\end{gathered}
$$

The corrected inclination $i_{k}$ and the inclination correction $\delta i_{k}$ are expressed by

$$
\begin{gathered}
i_{k}=i_{0}+d i / d t \Delta t_{k}+\delta i_{k} \\
\delta i_{k}=C_{i c} \cdot \cos \left(2 \Phi_{k}\right)+C_{i s} \cdot \sin \left(2 \Phi_{k}\right)
\end{gathered}
$$

Finally, the terrestrial coordinates of the satellite in Earth-centered, Earth-fixed (ECEF) $X_{i}, Y_{i}$ and $Z_{i}$ can be calculated using the following equations

$$
\begin{gathered}
X_{i}=X_{p} \cdot \cos \left(\Omega_{k}\right)-Y_{p} \cdot \cos \left(i_{k}\right) \cdot \sin \left(\Omega_{k}\right) \\
Y_{i}=X_{p} \cdot \sin \left(\Omega_{k}\right)-Y_{p} \cdot \cos \left(i_{k}\right) \cdot \cos \left(\Omega_{k}\right) \\
Z_{i}=Y_{p} \cdot \sin \left(i_{k}\right)
\end{gathered}
$$

where $\Omega_{k}$ is corrected longitude of the ascending node, expressed by

$$
\Omega_{k}=\Omega_{0}+\left(\dot{\Omega}-\omega_{e}\right) \cdot \Delta t_{k}-\omega_{e} \cdot t_{o e}
$$

where $\omega_{e}=7.2921151467 \cdot 10^{-5}$ is the angular velocity of Earth rotation. 
It is important to note that the positions are estimated at the transmission time $t^{s}$, while the reference in this work is the receiver time $t^{r}$. Note the satellite position can change as much as 300 meters from the time the signal was transmitted to the time the signal was received, approximately 0.07 seconds later. Thus, it is necessary to remember that the time relation can be expressed as

$$
t^{s}=t^{r}-\Delta t_{r}+\Delta t_{s}-\tau-\Delta t_{m}
$$

where $\Delta t_{r}(k)$ and $\Delta t_{s}(k)$ are the receiver and satellite clock errors, $\Delta t_{m}$ is the delay due to the medium (Ionospheric, tropospheric delay, etc.) and $\tau$ is the propagation time and can be represented as $\tau=\rho\left(t^{r}, t^{s}\right) / c$, the term $\rho\left(t^{r}, t^{s}\right)$ is the geometric distance estimated from receiver (at receive time) to the satellite (at transmit time) and $c$ is the velocity of light. Note that the pseudorange is represented by

$$
P R\left(t^{r}, t^{s}\right)=\rho\left(t^{r}, t^{s}\right)+c\left(\Delta t_{r}-\Delta t_{s}\right)+c\left(\Delta t_{m}\right)
$$

Starting with the receiver time $t^{r}$ the transmit time can be computed by an iterative algorithm known as "the light time equation," which can be written as follows

$$
\begin{aligned}
& t^{S}(0)=t^{r} \\
& t^{S}(1)=t^{r}-\frac{P R\left(t^{r}, t^{S}(0)\right)}{c} \\
& t^{S}(2)=t^{r}-\frac{P R\left(t^{r}, t^{s}(1)\right)}{c}
\end{aligned}
$$

where the satellite position and hence the geometric distance $P R\left(t^{r}, t^{S}\right)$ is calculated at each step using the Keplerian-type elements from the Navigation Message described above, and the algorithm is stopped once the computed range converges. 